(11) Nordic Council of Ministers

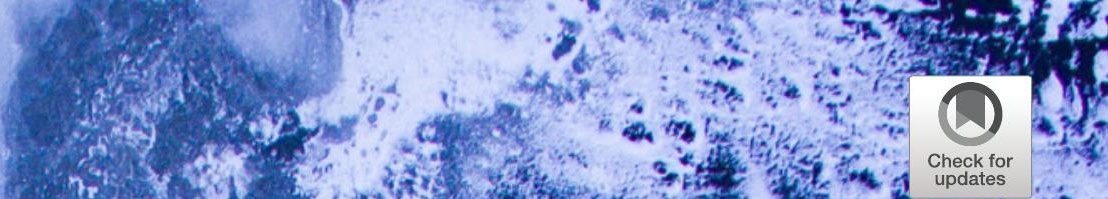

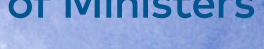





\section{Suspect screening in Nordic countries}

Point sources in city areas

Martin Schlabach, Peter Haglund, Malcolm Reid, Pawel Rostkowski, Cathrin Veenaas, Kine Bæek and Bert van Bavel

TemaNord 2017:561 


\section{Suspect screening in Nordic countries}

Point sources in city areas

Martin Schlabach, Peter Haglund, Malcolm Reid, Pawel Rostkowski, Cathrin Veenaas,

Kine Bæk and Bert van Bavel

ISBN 978-92-893-5199-7 (PRINT)

ISBN 978-92-893-5200-0 (PDF)

ISBN 978-92-893-5201-7 (EPUB)

http://dx.doi.org/10.6027/TN2017-561

TemaNord 2017:561

ISSN $0908-6692$

Standard: PDF/UA-1

ISO 14289-1

(c) Nordic Council of Ministers 2017

Cover photo: unsplash.com

Print: Rosendahls

Printed in Denmark

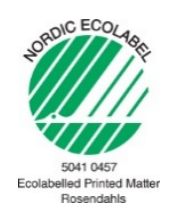

\section{Disclaimer}

This publication was funded by the Nordic Council of Ministers. However, the content does not necessarily reflect the Nordic Council of Ministers' views, opinions, attitudes or recommendations.

Rights and permissions

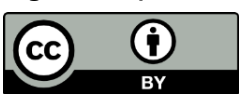

This work is made available under the Creative Commons Attribution 4.0 International license (CC BY 4.0) https://creativecommons.org/licenses/by/4.o.

Translations: If you translate this work, please include the following disclaimer: This translation was not produced by the Nordic Council of Ministers and should not be construed as official. The Nordic Council of Ministers cannot be held responsible for the translation or any errors in it.

Adaptations: If you adapt this work, please include the following disclaimer along with the attribution: This is an adaptation of an original work by the Nordic Council of Ministers. Responsibility for the views and opinions expressed in the adaptation rests solely with its author(s). The views and opinions in this adaptation have not been approved by the Nordic Council of Ministers. 
Third-party content: The Nordic Council of Ministers does not necessarily own every single part of this work. The Nordic Council of Ministers cannot, therefore, guarantee that the reuse of third-party content does not infringe the copyright of the third party. If you wish to reuse any third-party content, you bear the risks associated with any such rights violations. You are responsible for determining whether there is a need to obtain permission for the use of third-party content, and if so, for obtaining the relevant permission from the copyright holder. Examples of third-party content may include, but are not limited to, tables, figures or images.

Photo rights (further permission required for reuse)

Any queries regarding rights and licences should be addressed to:

Nordic Council of Ministers/Publication Unit

Ved Stranden 18

DK-1061 Copenhagen K

Denmark

Phone +4533960200

pub@norden.org

\section{Nordic co-operation}

Nordic co-operation is one of the world's most extensive forms of regional collaboration, involving Denmark, Finland, Iceland, Norway, Sweden, and the Faroe Islands, Greenland and Åland.

Nordic co-operation has firm traditions in politics, economics and culture and plays an important role in European and international forums. The Nordic community strives for a strong Nordic Region in a strong Europe.

Nordic co-operation promotes regional interests and values in a global world. The values shared by the Nordic countries help make the region one of the most innovative and competitive in the world.

The Nordic Council of Ministers

Nordens Hus

Ved Stranden 18

DK-1061 Copenhagen K, Denmark

Tel.: +4533960200 www.norden.org

Download Nordic publications at www.norden.org/nordpub 



\section{Contents}

Preface .7

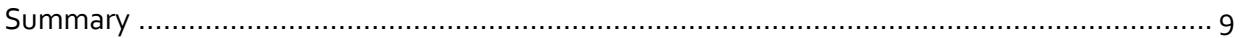

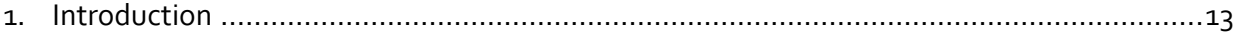

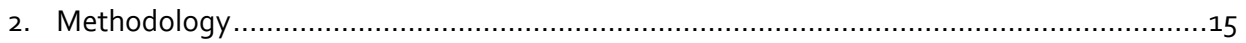

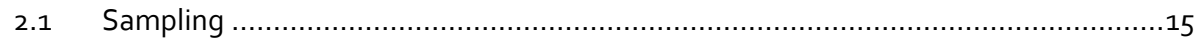

2.2 Chemical Laboratory Work ................................................................... 17

2.3 Compound identification (Post-acquisition Data Treatment) ................................21

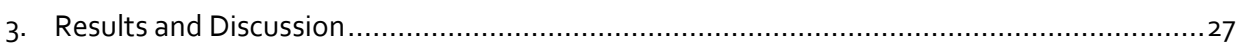

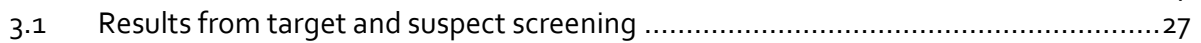

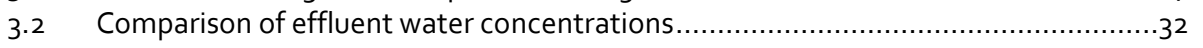

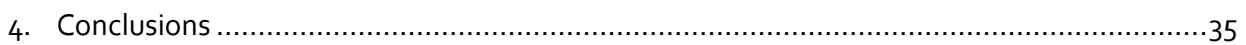

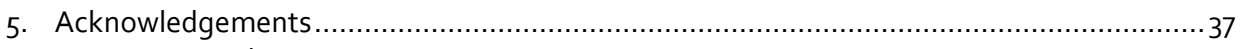

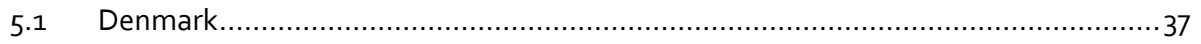

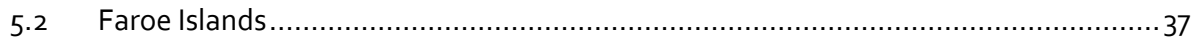

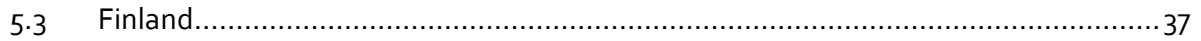

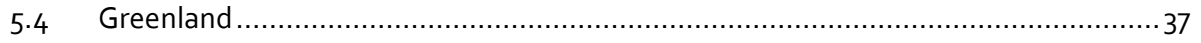

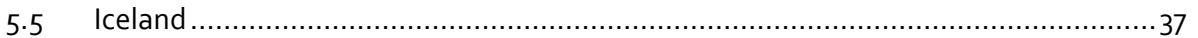

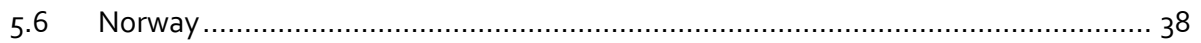

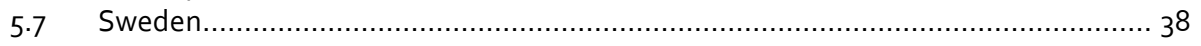

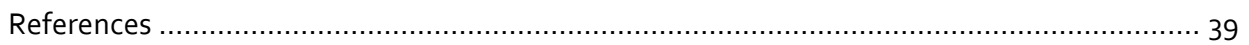

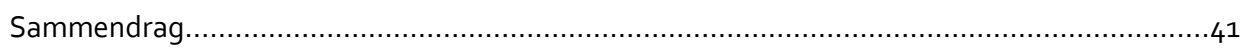

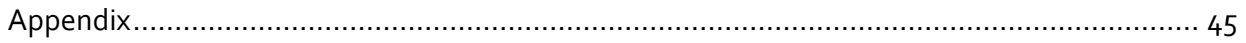





\section{Preface}

The aim of the Nordic environmental screening is to obtain a snapshot of the occurrence of potentially hazardous substances, both in regions most likely to be polluted as well as in some pristine environments. The focus is on less known, anthropogenic substances and their derivatives, which either are used in high volumes or are likely to be persistent and hazardous to humans and other organisms.

The project steering group has initiated a non-targeted screening of possible environmental pollutant in selected samples from point-sources in cities in 2015. The method "non-target screening", as defined in the invitation for tender, is a technique that can identify environmental pollutants without a preceding selection of the compounds of interest. The Nordic screening group wants to use the non-target screening method to discover emerging pollutants in the Nordic environment-impact both from small cities as in Greenland and larger cities as in Sweden. The result is expected to be used for later targeted studies and in international chemical regulation processes like REACH and the Stockholm POP convention.

The following Nordic countries and self-governing areas are included in the project: Finland, Sweden, Norway, Denmark, Faroe Islands, Iceland and Greenland.

The matrices selected for the analyses are:

- Effluent water from waste water lines or treatment plants.

- Sediments from receiving waters (whether marine or freshwater as decided by each country).

- Fish from receiving waters (marine or freshwater as decided by each country).

The Nordic screening project is run by a steering group with representatives from Danish Centre for Environment and Energy, Aarhus University, Denmark, Finnish Environment Institute, Environment Agency of Iceland, the Environment Agency of the Faroe Islands, the Norwegian Environment Agency, Greenland Institute of Natural Resources and the Swedish Environmental Protection Agency. The project is financed and supported by the Nordic Council of Ministers through the Nordic Chemicals Group and the Marin Group (HAV) as well as the participating institutions.

The chemical analyses have been carried out jointly by NILU-Norwegian Institute for Air Research, Norwegian Institute for Water Research (NIVA), and Umeå University Department of Chemistry. The respective participating Nordic countries organised sample selection, collection and transport of samples based on a sample protocol and manuals provided by the analytical laboratories. 



\section{Summary}

On behalf of the Nordic Council of Ministers through the Nordic Chemicals Group, and the Marin Group (HAV) NILU-Norwegian Institute for Air Research, Norwegian Institute for Water Research (NIVA), and Umeå University Department of Chemistry jointly performed a suspect screening study in effluent, sediment, and fish samples from all seven Nordic countries.

This study was original planned as non-target screening. However, as the nontarget screening approach is under rapid development and different data treatment approaches are tested, it became necessary to distinguish clearly between the different approaches and "suspect screening" is coming up as a new term or concept. The original concept non-target screening is now replaced by two concepts, namely suspect screening and non-target screening in a more strict definition, and only the time efficient suspect screening approach was applied here.

The respective participating Nordic countries organised sample selection, collection and transport of samples based on a sample protocol and manuals provided by the analytical laboratories. Each country sampled one effluent water sample (single/grab sample) from a major sewage treatment plant. In addition, it was also sampled one sediment sample (single/grab sample) and one fish sample (pooled sample) each. These environmental samples were taken close to the effluent emission samples except the Danish samples of sediment and fish. For this study a generic extraction and clean-up of samples was carried out at NIVA, Oslo, Norway. To cover a broadest possible range of compound groups two different extraction and clean-up methods were applied. The first method is optimized for non-polar and very lipophilic compounds like PCBs, PAHs and other classical POPs, and the second is optimized for polar compounds like pharmaceuticals, modern pesticides and biocides, PFAS, and bisphenols. Prior to extraction, samples were spiked with a number of isotopically labelled internal standards. The final extracts were sent to the different laboratories of the project group.

To cover a broad spectra of different compounds both GC- and LC-MS was applied (1. GCXGC-HRMS, 2. GC-ECNI-HRMS, 3. LC-OToFMS ESI+, and 4. LC-OToFMS ESI-). Raw data from all analyses in the project (in the format native to the instrument it was obtained) is being stored at NILU datacenter at secured, fully backed up FTP server. In a first step of raw data treatment important information of the mass spectrometric peaks as the mass centroid positions of the peaks, their areas under curve and fullwidth-at-half-maxima are extracted from the raw MS data and stored for further treatment. The next and most efficient step of post acquisition data treatment is normally filtration of the list of mass spectra of interest against libraries of suspect compounds. These libraries are either home-made, from commercial supplies or from the NORMAN network and are the most critical part of the suspect screening approach. 
Suspect screening leads directly to tentative candidates with evidence for possible structure(s), but insufficient information for the exact structure.

It was possible to identify and partly (semi)quantify: Per- and polyfluorinated compounds (PFC), chlorinated and brominated compounds, different flame retardants, bisphenols, polycyclic aromatic compounds (PAC), industrial additives like UVstabilizers, antioxidants, and plasticizers, and pharmaceuticals and personal care products (PPCP). When identified by GC the concentration was estimated in a semiquantitative way. However, today it is not yet possible to estimate concentrations, when using LC-MS technology.

There are identified several compounds, which might be interesting and relevant for further in depth studies:

- Emerging PFAS compounds like 4:2 FTMAC (1799-84-4), 4:3 Acid (80705-13-1), C6/C6-PFPIA (40143-77-9), FOSA (754-91-6), 4:2 FTAL (135984-67-7), and 4:2 FTOH (2043-47-2) were identified in sediments.

In the sediment sample from Sweden several chlorinated aromatic compounds were found: 3,4,5-trichlorobenzenamine (634-91-3), 1-chloro-3-isocyanatobenzene (290938-8), and dichloro and trichloroaniline (CAS 608-27-5 and 634-91-3). Several substituted aniline derivatives are used in the production of dyes and herbicides and these findings may be related to emissions from industrial processes.

Several new bisphenols ( $S, E$, and $A F)$ were identified, and most concerning even in fish samples.

Numerous industrial additives were found in sediment, water and fish samples. These were for instance phenols, UV-stabilizers, antioxidants, and plasticizers of phthalate-type. Also a benzothiazole compound (2-methylthiobenzothiazole, MTBT, CAS: 615-22-5) was frequently found in all sample types and in high concentrations. 2Methylthiobenzothiazole is a degradation product of mercaptobenzothiazol (MBT, CAS: 149-30-4), which is used in vulcanization of rubber. MTBT has earlier been detected in other screening studies in water and other abiotic samples, however, to our best knowledge not yet in marine biota.

A huge number of pharmaceuticals were identified in effluent samples. Many of the identified pharmaceuticals are not only found in effluents from sewage treatment plants (STPs) with basic or no treatment, but also in STPs with advanced treatment technologies. The effluent water samples are similar enough to allow a comparison of contaminant levels. The concentrations in effluents from STPs with advanced sewage treatment (SE, DK, $\mathrm{Fl}, \mathrm{NO}$ ) vs. STPs with basic or no treatment (FO, IS, GL) is likely to reflect the relative persistency of relatively water soluble contaminants. The terpenoids coumarin, carvone, menthol and tetrahydralinalol and the alkaloid caffeine are, for instance, much less abundant in effluent from STPs with advanced treatment. It is plausible that those are degraded in the activated sludge process. However, for a number of contaminants like m-Cresol, 2-(Methylthio)pyridine, 2(Methylthio)benzothiazole, 9-methylacridine, 3,3-Diphenylacrylonitrile, 3,3Diphenylpropionitrile, N-butyl-benzenesulfonamide, and Diclofenac the levels were 
highest in effluent from one of the STPs in SE, DK or Fl, likely because of higher load from industry, traffic and other urban sources.

With an initial suspect data treatment using different mass spectral data bases it was possible to detect and identify a surprisingly long list of compounds of possible emerging concern with a level of confidence of three or better. When evaluating the total outcome of this study, it is important to keep the following limitations in mind: In order to get a wide overview over all anthropogenic compounds in environmental samples, a general sample preparation method has to be chosen. Many compounds, which easily could be detected by a dedicated target method, will be masked or lost either by (1) interfering matrix, (2) instrumental overload/saturation, and/or (3) possible loss of compounds. Furthermore, the applied GC/LC methods are often not optimal for each single analyte. For the time being, suspect/non-target screening cannot replace dedicated target methods in sensitivity and specificity, but it has proven to be an important tool for identification of compounds of emerging concern. 



\section{Introduction}

Today, the monitoring of the environmental level of organic potentially hazardous compounds is mainly based on the use of mass spectrometers. The applied techniques can be classified either as target or non-target methods, that means either selecting the compounds of interest before starting the analysis or at a later stage. Traditional environmental monitoring of organic pollutants as PCB or chlorinated pesticides is targeting specific compounds, and is therefore named target analysis. In contrast, nontarget analysis or non-target screening is defined as an analytical technique that can identify environmental pollutants without selection of the compounds of interest before starting the chemical analysis. This understanding of non-target screening is also at the base of the invitation to tender of this project.

Target analysis starts with optimizing the sample extraction and clean-up, and instrumental method to the beforehand selected analytes. This means that the complete analytical workflow including extraction, clean-up, and detection are optimized to provide a specific and accurate measurement (Thomas et al., 2015). Most of the target analytical methods are quantitative, a feature that is often facilitated by using isotopically labeled internal standards that are analogues of the target analytes.

Non-target analysis has always existed in parallel to target analysis. However, the earlier instruments were not appropriate for applying non target analysis on a routine base and sufficient sensitivity. Over the past years, mass spectrometers used for environmental analysis have developed considerably. The major revolution in instrument technology is a new generation of accurate mass or high-resolution mass spectrometers (HRMS), which now allow the acquisition of a full spectrum of compounds with the same sensitivity which earlier were possible with only some very few selected compounds. These new instruments widens the applicability of non-target screening also onto environmental samples.

Both suspect and non-target screening are using HRMS and are complementary to targeted analysis, as shown and explained in (Figure 3) and chapter 2.3.

Similarly to target analysis, suspect screening uses some form of prior knowledge to search for the presence of a substance in a sample, however, without the use of a reference standards. Instead the exact mass, isotope pattern and chromatographic retention time is used. There are a large number of different databases and libraries available that can be used to perform the suspect screening process, such as those sold by instrument producers and those in the public domain, such as STOFF-IDENT (https://www.Ifu.bayern.de/stoffident/\#!home), MassBank (https://massbank.eu/MassBank/) or ChemSpider (http://www.chemspider.com/). A prioritized list of compounds, which one would expect to find in the environment, is essentially the most effective suspect list for environmental screening. This list must be supported with the necessary information to identify the compounds in accurate mass 
full-scan chromatograms. The Norman Network is working towards creating a common suspect screening list through exchanging information and this will be freely available (http://www.norman-network.com/?q=node/236).

Non-target screening in the new and stricter understanding, involves the identification of peaks in the chromatogram that are unknown and about which no prior information is known. This typically involves the selection of peaks (that have not been identified by suspect or target analysis) based upon their intensity (size) and the absence in control/blank samples. Each peak is then identified based on the accurate mass measurement that is used to generate the most plausible molecular formula, a process that is often complimented by the use of MS/MS fragment data. Such data can then be compared with libraries and/or various in silico fragmentation platforms to identify candidates.

Any screening without reference standards carries a level of uncertainty. Schymanski et al., have proposed a matrix, which connects the different identification approaches versus the confidence in identification levels. A slightly adapted version of this matrix is shown in Figure 3, which in contrast to the matrix of Schymanski, also show the traditional target analysis approach. A high quality target method is normally based on the use of calibration or reference standards and internal standards and will end up at level o, which is not included in the Schymanski matrix. For a compound at confidence level o the chemical structure is confirmed, and in addition also the concentration is known. At level 1 (the best level in the Schymanski matrix) only the structure is determined by comparison to an authentic reference standard and confirmation with MS, MS/MS and retention time matching. Level 2 is described as the possible structure provided by a match with library spectra and/or other diagnostic evidence, and level 3 as a tentative candidate based upon evidence for a possible structure, but where there is insufficient information for identification to exact structure. Level 4 describes the unequivocal molecular formula and level 5 the exact mass of the molecular ion. For the purpose of this report, only compounds identified with a confidence of level o to 3 are reported. 


\section{Methodology}

\subsection{Sampling}

Co-ordination of sampling was supervised by NIVA, whereas the practical work was done or coordinated by the seven participating Nordic institutions in accordance with the Sampling Protocol as shown in the Appendix. This sampling protocol was prepared by the contracted institutes and adopted by the Steering Group. Each country sampled one effluent water sample (single/grab sample) from a major sewage treatment plant. In addition, it was also sampled one sediment sample (single/grab sample), and one fish sample (pooled sample) each (Table 1). These environmental samples were taken close to the effluent emission samples except the Danish samples of sediment and fish (see also Table 2).

Figure 1: Sampling stations

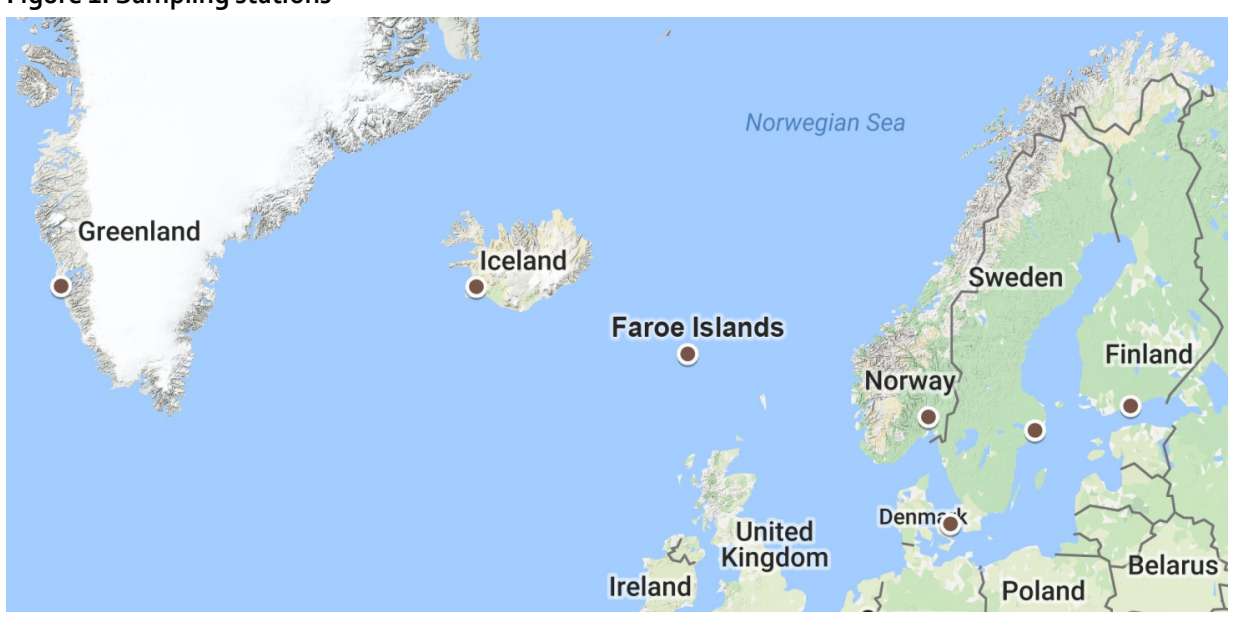

Note: Sampling of effluent water, sediment, and fish samples was done by the national collaborators.

Source: Google Maps. 
Table 1: Sampling stations

\begin{tabular}{|c|c|c|c|c|c|c|}
\hline Matrix & $\begin{array}{l}\text { Sample } \\
\text { ident. }\end{array}$ & Location & Site & Latitude & Longitude & $\begin{array}{l}\text { Number of } \\
\text { subsamples }\end{array}$ \\
\hline Effluent & FO-1-Eff & Torshavn & UA 11 (Sersjantvikin) & 62.00783 & -6.76132 & 1 \\
\hline Sediment & FO-1-Sed & Torshavn & UA 17 (Alakeri) & 62.00225 & -6.77287 & 1 \\
\hline Fish Coalfish & FO-1-Fis & Torshavn & UA11 og UA17 & 62.00225 & -6.77287 & 20 \\
\hline Effluent & DK-1-Eff & Aarhus & Marselisborg WWTP & 56.23400 & 10.34995 & 1 \\
\hline Sediment & DK-1-Sed & Roskilde & Ros 60 & 55.70783 & 12.06667 & 1 \\
\hline Fish Eel pout & DK-1-Fis & Roskilde & Risø & 55.68833 & 12.07500 & 28 \\
\hline Effluent & FI-1-Eff & Helsinki & Viikinmäki & 60.22670 & 24.99645 & 1 \\
\hline Sediment & $\mathrm{Fl}-1-\mathrm{Sed}$ & Helsinki & Vanhankaupunginselkä & 60.20083 & 24.99463 & 1 \\
\hline Fish Perch & $\mathrm{Fl}-1$-Fis & Helsinki & Vanhankaupunginselkä & 60.20083 & 24.99463 & 23 \\
\hline Effluent & SE-1-Eff & Stockholm & Henriksdal WWTP & $59 \cdot 30975$ & 18.10763 & 1 \\
\hline Sediment & SE-1-Sed & Stockholm & Henriksdal WWTP & $59 \cdot 32025$ & 18.13525 & 1 \\
\hline Fish Perch & SE-1-Fis & Stockholm & Henriksdal WWTP & $59 \cdot 32025$ & 18.13525 & 8 \\
\hline Effluent & GL-1-Eff & Nuuk & Kakillarnat & 64.19687 & -51.70252 & 1 \\
\hline Sediment & GL-1-Sed & Nuuk & Kakillarnat & 64.19692 & -51.70575 & 1 \\
\hline Fish Cod & GL-1-Fis & Nuuk Fiord & Kakillarnat & 64.19748 & -51.70528 & 5 \\
\hline Effluent & IS-1-Eff & Reykjavik & Klettagardar WWT & 64.15553 & -21.87275 & 1 \\
\hline Sediment & IS-1-Sed & Reykjavik & Inner Faxafloi bay & 64.19228 & -21.92260 & 1 \\
\hline Fish Cod & IS-1-Fis & Reykjavik & Inner Faxafloi bay & 64.19228 & -21.92260 & 5 \\
\hline Effluent & NO-1-Eff & Oslo & VEAS & 59.79319 & 10.49958 & 1 \\
\hline Sediment & NO-1-Sed & Oslo & Oslofjord & 59.79018 & 10.51922 & 1 \\
\hline Fish Cod & $\mathrm{NO}-1-\mathrm{Fis}$ & Oslo & Oslofjord & 59.79018 & 10.51922 & 5 \\
\hline
\end{tabular}

Note: Station coordinates are given as WGS84 geographic coordinates in decimal degrees.

The biota sample of each country is one pooled samples, where the number of subsamples are listed in the table.

Effluent from Faroe Islands were sampled at the Sersjantvikin WWTP, Torshavn. The Sersjantvikin WWTP, Torshavn, receives domestic wastewater only and from approx. 1,000 pe. This WWTP may be described as consisting of a primary purification step (Kaj, Wallberg, \& Brorström-Lundén, 2014).

Effluent from Denmark were sampled at the Marselisborg WWTP, Aarhus. The Marselisborg WWTP, Aarhus, receives domestic, industrial and hospital wastewater from approx. 200,000 pe. This WWTP may be described as state-of-the art WWTP.

Effluent from Finland were sampled at the Viikinmäki WWTP, Helsinki. The Viikinmäki WWTP receives domestic, industrial and hospital wastewater from approx. 800,000 pe. The treatment process in Viikinmäki wastewater treatment plant is based on an activated sludge method and it has three phases: mechanic, biological and chemical treatment.

Effluent from Sweden were sampled at Henriksdal, WWTP, receiving waste water from up to 1 million p.e. The treatment process at Henriksdal wastewater treatment plant is based on an activated sludge method and it has three phases: mechanic, biological and chemical treatment.

Effluent from Greenland was sampled at a sewage drain at Kakillarnat in the northern part of Nuuk. The wastewater is not treated and is mainly domestic and some industrial waste water.

Effluent from Island was sampled at the Klettagardar WWTP. The waste water is treated mechanically and is domestic, industrial, and hospital waste water. 
The effluent sample from Norway were taken at VEAS, WWTP, Asker receiving waste water from up to 700,000 p.e. The treatment process at VEAS wastewater treatment plant is based on an activated sludge method and it has three phases: mechanic, biological and chemical treatment.

Table 2: Waste water lines used where effluent samples were taken

\begin{tabular}{lllrr} 
Sample ident. & Location & Site & Water (m3/year) & Population \\
FO-1-Eff & Torshavn & UA 11 (Sersjantviken) & & \\
DK-1-Eff & Aarhus & Marselisborg WWTP & 10 Million & 202000 \\
FI-1-Eff & Helsinki & Viikinmäki & 101 Million & 800000 \\
SE-1-Eff & Stockholm & Henriksdal WWTP & 90 Million & 1000000 \\
GL-1-Eff & Nuuk & Kakillarnat & n.a. & 5000 \\
IS-1-Eff & Reykjavik & Klettagardar WWT & 1 Million & 100000 \\
NO-1-Eff & Oslo & VEAS & 107 Million & 700000 \\
\hline
\end{tabular}

\subsection{Chemical Laboratory Work}

For "traditional" target analysis the chemical work is often extremely specialized. Extraction and cleanup methods are optimized towards a small group of compounds. The mass spectrometer used for identification and quantification is normally set to the Selected Ion Monitoring mode (SIM). This gives optimal sensitivity, however, only signals from the target compounds are registered and signals from other compounds are not registered at all. For non-target analysis it is necessary to open up for different compound groups, and more general extraction and cleanup methods are chosen. In addition, the mass spectrometer is set to full scan mode, which means that it registers all mass signals in a given range (see also figure 3, page 21). This non-specialized approach leads to the following limitations: Many compounds, which easily could be detected by a dedicated target method, will be masked or lost either by (1) interfering matrix, (2) instrumental overload/saturation, and (3) possible loss of compounds. Furthermore, the applied GC/LC methods are often not optimal for each single analyte.

For this study a generic extraction and clean-up of samples was carried out at NIVA, Oslo, Norway. All samples were sent to NIVA and stored in freezer at $-20^{\circ} \mathrm{C}$ before extraction. To cover a broadest possible range of compound groups two different extraction and clean-up methods were applied. The first method is optimized for nonpolar and very lipophilic compounds like PCBs, PAHs and other classical POPs, and the second is optimized for polar compounds like pharmaceuticals, modern pesticides and biocides, PFAS, and bisphenols. Prior to extraction, samples were spiked with a number of isotopically labelled internal standards. The final extracts were sent to the different laboratories of the project group. 


\subsubsection{Sample extraction and clean-up}

A mix of deuterated and ${ }^{13} \mathrm{C}$-labelled pharmaceuticals, bisphenols, PFCs, and BFRs were used as an internal standard for all samples matrices and added before extraction.

\section{Effluent water}

Samples of effluent water were filtrated on Spin-X filters and extracted on Oasis HLB (200 $\mathrm{mg}$ ) solid phase extraction cartridges. After washing the cartridge with MilliQ water and MilliO water with $5 \%$ methanol, the analytes were eluted with $2 \%$ formic acid in methanol, methanol, and $2 \%$ ammonium hydroxide in methanol. Extracts were evaporated under nitrogen and reconstituted in toluene for GC-MS analysis and methanol for LC-MS analysis.

\section{Sediment}

Sediment samples were freeze-dried and $2 \mathrm{~g}$ dry sediment was extracted twice by ultrasonic extraction with dichloromethane. To remove elemental sulphur (S8), which would interfere and corrupt MS-detection, activated copper powder was added to the extracts. ${ }^{1}$ For GC-MS analysis no further cleaning was applied and the solvent was exchanged to tolvene. For LC-MS analysis the solvent was exchanged to acetonitrile, and the extracts were "washed" twice with n-hexane.

Fish

Between 1 and $4 \mathrm{~g}$ fish liver was shaken in $1 \mathrm{~h}$ and treated in a ultrasonic bath for 30 min in a solution of acetonitrile, milliQ water, and n-hexane. After centrifugation the nhexane phase and acetonitrile phase were separated. For GC-MS analysis the n-hexane phase was filtered and further cleaned on a GPC-column. For LC-MS analysis the extracts were filtered.

\subsubsection{Full scan MS analysis}

To cover a broad spectra of different compounds both GC- and LC-MS was applied (see figure 2). The non-target and suspect screening were performed at NILU and NIVA, Norway and UmU, Sweden.

\footnotetext{
${ }^{1}$ This sulphur removal is not limited to elemental sulphur, and will also remove organic sulfides like diphenyl disulfide and
} methionine, but not PFOS and other sulfonic acids. 


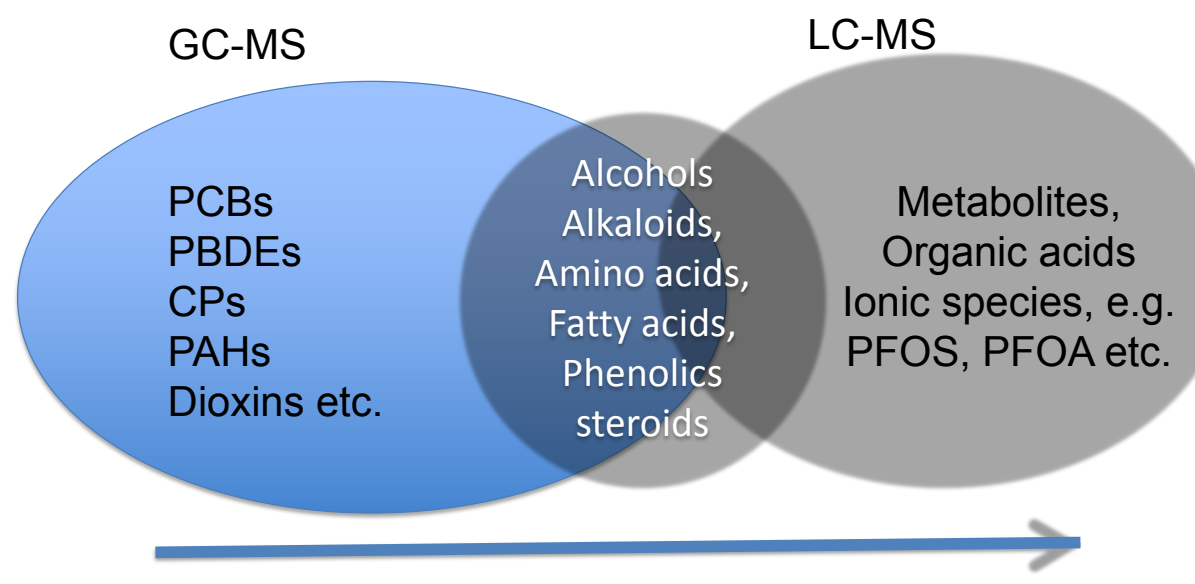

POLARITY

Source: NILU.

Full scan analysis with GCxGC-HRMS

Prior to analysis, all samples were concentrated to $\sim 150 \mu \mathrm{L}$ sample volume. The extracts were injected into an Agilent $7890 \mathrm{~N}$ GC system equipped with a secondary oven and modulator coupled to a Leco high resolution time-of-flight mass spectrometer (GCxGC-HRMS).

The following GC columns were used:

- 1st oven: $30 \mathrm{~m} \mathrm{Rtx-5MS}(250 \mu \mathrm{m} \times 0.25 \mu \mathrm{m})+1.29 \mathrm{~m}$ Rxi-17Sil MS (250 mm x $0.25 \mu \mathrm{m})$.

- Modulator: $0.1 \mathrm{~m}$ Rxi-17Sil MS, quad jet two stage modulator.

- 2nd oven: 0.56 m Rxi-17Sil MS + 0.4 m uncoated apolar deactivated silica column (Supelco).

- Transferline: 0.6 m uncoated apolar deactivated silica column (Supelco).

GC-MS settings:

- Injection: $1 \mu \mathrm{L}$ in pulsed splitless mode.

- Carrier gas: Helium at $1 \mathrm{~mL} / \mathrm{min}$.

- $\quad$ 1st oven: $90^{\circ} \mathrm{C}(2 \mathrm{~min}), 5^{\circ} \mathrm{C} / \mathrm{min}, 300^{\circ} \mathrm{C}$ (11 min).

- $\quad$ 2nd oven: $105^{\circ} \mathrm{C}(2 \mathrm{~min}), 5^{\circ} \mathrm{C} / \mathrm{min}, 300^{\circ} \mathrm{C}(14 \mathrm{~min})$.

- Modulator: temperature offset $+15^{\circ} \mathrm{C}$, Modulation period 3.5 $\mathrm{sec}$ (hot pulse 1.19 sec, cold time $0.56 \mathrm{sec}$ ).

- Transferline: $325^{\circ} \mathrm{C}$. 
- MS: El positive mode, high resolution, Acquisition rate 150 spectra/sec, mass range $\mathrm{m} / \mathrm{z} 38-600$, ion source temperature $250^{\circ} \mathrm{C}$.

Full scan analysis with GC-ECNI-MS

Prior to analysis, all samples were concentrated to $\sim 150 \mu \mathrm{L}$ sample volume. The extracts were injected into an Agilent $7890 \mathrm{~N}$ GC system coupled to an Agilent 7200 QToF mass spectrometer operated in electron capture negative ionization mode (GC-ECNI-HRMS).

The following GC-MS parameters were applied:

- $1 \mu \mathrm{L}$ pulsed split-less injection, 20 psi for $1 \mathrm{~min}$

- $30 \mathrm{~m} \times 0.25 \mathrm{~mm} \times 0.25 \mu \mathrm{m} \mathrm{DB} 5 \mathrm{~ms}-\mathrm{UI}$

- He $0.8 \mathrm{~mL} / \mathrm{min}$, constant flow

- $\quad 90^{\circ} \mathrm{C}(1 \mathrm{~min})-4^{\circ} \mathrm{C} / \mathrm{min}-310^{\circ} \mathrm{C}$ (2 min)

- $\mathrm{ECNI}$, methane $(40 \%$ flow)

- 29-1000 amu, $2 \mathrm{~Hz}$

- High-res mode

- MassHunter, Qual analysis

Full scan analysis with LC-HR-QToF in positive ESI-mode

The analytes were separated on an Acquity UPLC (Waters, Norway) using an Acquity BEH C18 column ( $100 \times 2.1$ mm, $1.7 \mu \mathrm{m}$ ) (Waters, Norway) with a methanol and water (10 $\mathrm{mM}$ ammonium acetate) mobile phase. Gradient elution was from $2 \%$ to $99 \%$ methanol over a 13-minute program. The UPLC system was connected to a mass spectrometer (Xevo G2S OToF, (Waters, Norway)) operated in positive electrospray ionisation mode.

Full scan analysis with LC-HR-OToF in negative ESI-mode

The analytes were separated on an Agilent 1290 UHPLC using a Waters Acquity HSS T3 column $(150 \times 2.1 \mathrm{~mm}, 1.8 \mu \mathrm{m})$ with methanol and water mobile phase. Gradient elution was from $0 \%$ to $100 \%$ methanol over a 25 minutes program. The UPLC system was connected to a mass spectrometer (Agilent 6550 OToF), operated in negative electrospray ionisation mode.

\subsubsection{Storage of raw data}

Raw data from all analyses in the project (in the format native to the instrument it was obtained) is being stored at NILU datacentre at secured, fully backed up FTP server. 


\subsection{Compound identification (Post-acquisition Data Treatment)}

\subsubsection{Peak picking}

Both single ion monitoring (SIM) and especially full scan analyses on high resolution mass spectrometers obtain and register an enormous amount of information. A first step of data reduction is the MS-peak picking algorithm (see details in chapter 2.3.4). In this first step of raw data treatment important information of the mass spectrometric peaks as the mass centroid positions of the peaks, their areas under curve and full-width-at-halfmaxima are extracted from the raw MS data and stored for further treatment.

\subsubsection{Further post-acquisition work}

In parallel with the development of the advanced analytical instruments and software, advanced workflows for an efficient data treatment of full-scan high resolution MS and MSMS-data are a subject of a continuous development. The NORMAN network (http://www.norman-network.net/), which is the driving force in this development, has initiated an interlaboratory study (ILS) (Schymanski et al., 2015). One of the major outcomes of this ILS was a general consensus to use a workflow protocol proposed by EAWAG (Schymanski et al., 2014) for acquisition and data treatment. A slightly revised version of this workflow is shown in Figure 3.

Figure 3: Chemical laboratory and post-acquisition data treatment workflow in target and suspect/nontarget analysis

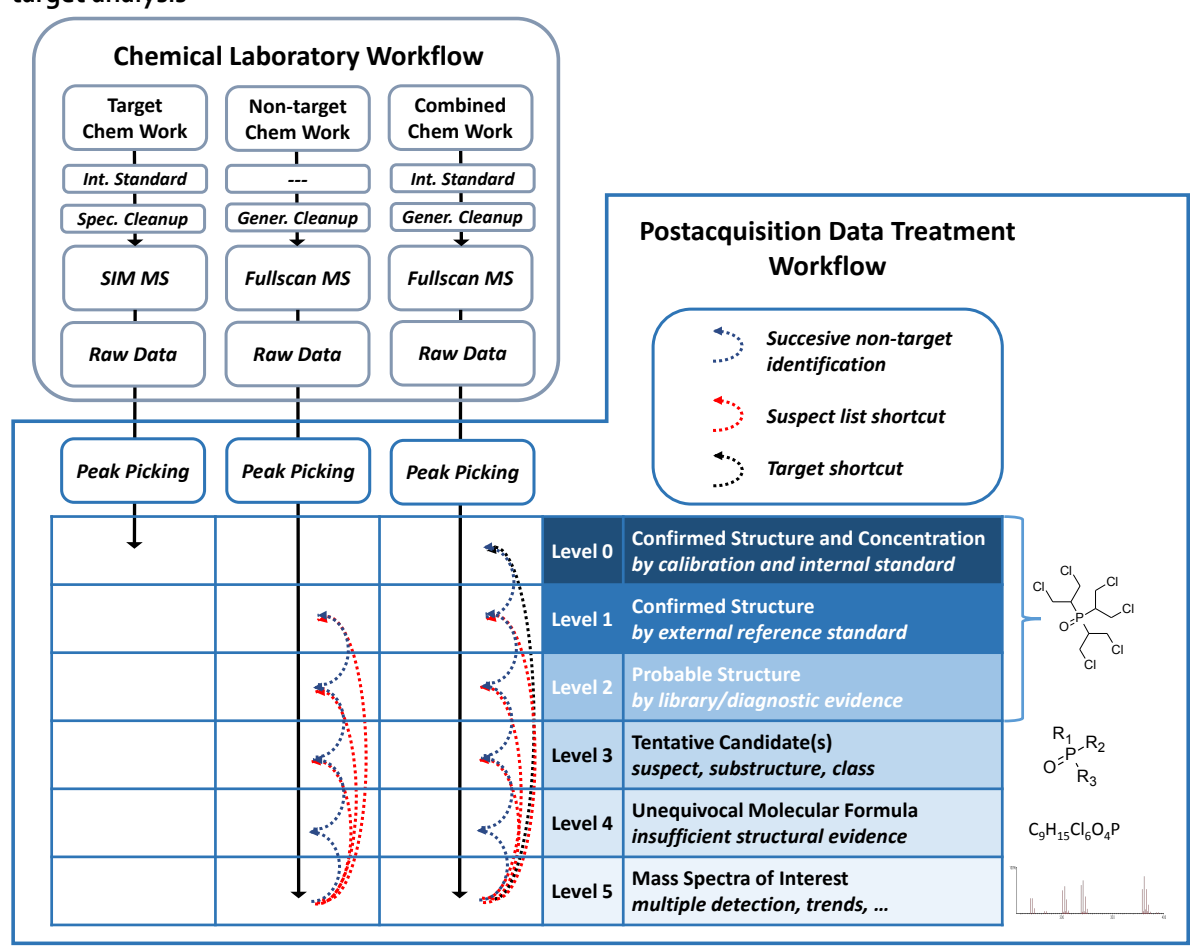

Source: NILU based on (Schymanski et al., 2015). 
Table 3: Important terms in target and suspect/non-target analysis

\begin{tabular}{|c|c|}
\hline Term & Explanation \\
\hline $\begin{array}{l}\text { Chemical Laboratory } \\
\text { Workflow }\end{array}$ & Sample extraction, cleanup, MS-acquisition, and raw data storage \\
\hline Internal standard & Labeled reference standard added before extraction, used for quantification purposes \\
\hline Specific cleanup & $\begin{array}{l}\text { Removal of disturbing sample matrix, specially adapted to a single group of target } \\
\text { compounds }\end{array}$ \\
\hline Generic cleanup & Removal of disturbing sample matrix, adapted to a wide range of different compounds \\
\hline SIM MS & $\begin{array}{l}\text { Selected ion monitoring: Mass spectrometric data acquisition limited to some selected ions } \\
\text { of a limited number of target compounds }\end{array}$ \\
\hline Fullscan MS & Continuous mass spectrometric data acquisition over a large mass range \\
\hline Raw data & $\begin{array}{l}\text { MS-data in a format as generated by the GC- or LC-MS containing information on } \\
\text { instrumental parameters and recorded intensity, retention time and mass spectra }\end{array}$ \\
\hline $\begin{array}{l}\text { Postacquisition Data } \\
\text { Treatment Workflow }\end{array}$ & $\begin{array}{l}\text { Data treatment performed on the acquired raw data including peak picking, prioritizing mass } \\
\text { spectra of interest, identification steps, and suspect list filtration }\end{array}$ \\
\hline $\begin{array}{l}\text { Successive non-target } \\
\text { identification }\end{array}$ & $\begin{array}{l}\text { Non-target identification starting without prior information from the exact mass, isotope, } \\
\text { adduct, and fragmentation information }\end{array}$ \\
\hline Suspect list shortcut & $\begin{array}{l}\text { Selecting and identifying compounds using prior information as libraries of suspect } \\
\text { compounds and other relevant sources }\end{array}$ \\
\hline Target shortcut & Identification and normally also quantification by the use of in-house reference compounds \\
\hline Level o & Confirmed Structure and Concentration by calibration and internal standard \\
\hline Level 1 & Confirmed Structure by external reference standard \\
\hline Level 2 & Probable Structure by library/diagnostic evidence \\
\hline Level 3 & Tentative Candidate(s) suspect, substructure, class \\
\hline Level 4 & Unequivocal Molecular Formula insufficient structural evidence \\
\hline Level 5 & Mass Spectra of Interest multiple detection, trends etc. \\
\hline
\end{tabular}

\subsubsection{Target analysis}

As shown in figure 3 target analysis can be performed in two different ways, either (1) with a traditional approach applying dedicated extraction and cleanup and dedicated mass spectrometry based on single ion monitoring method, or (2) with new combined approach applying more general extraction and cleanup and full scan mass spectrometry. For the time being the traditional approach is still giving better sensitivity and much better control of analytical quality. 
The most advanced target methods as dioxin or PCB analysis are normally ending up at confidence level o (Confirmed structure and concentration) as shown the above scheme (table 3). However, in some cases target analysis can also end up in Level 1 or Level 2 results. Level o or Confirmed structure and concentration is close to an ideal situation. Here the proposed structure can been confirmed by correlation to a reference standard with MS, MS/MS and retention time matching and calibration with reference and internal standards for quantification.

The following quality criteria were normally used to ensure correct identification and quantification of the target compound:

- The retention times should match those of the standard compounds within $\pm 0.1 \mathrm{~min}$.

- The signal-noise ratios are greater than 3:1.

- When a target compound is present in both blank and real samples, it will be reported only if the concentration in the sample is 10 times higher than in the blank.

- Mass error $<5 \mathrm{ppm}$.

- MS (/MS) spectra matches up MS (/MS) spectra of pure analytical standards.

- Use of internal standards for measurement of recovery in each single sample and calibration standards for calculation of targeted analyte concentrations.

- For each sample type laboratory blanks followed the sample preparation and quantification procedure as the regular samples to assess background interferences and possible contamination of the samples.

Quantification of target compounds is based on comparison of the compound's peak area to a calibration curve, which is generated in separate runs by injection of calibration solutions of a pure analytical standard of this compound. An internal standard is added in a known amount to the sample and the calibration standards. This standard is used for calibration by calculating the ratio of the signals of the analyte to the internal standard. With this approach loss of the analyte during sample preparation and injection is automatically compensated. The internal standard is a compound that is very similar, but not completely identical to the analyte in the samples, and it is presumed that the internal standard(s) behave like the analyte(s). For isotopically labeled internal standards this presumption is normally valid, but also other analytical standards can fulfill this requirement. 


\subsubsection{Suspect screening}

Non-target identification in the meaning of identification of compounds without any prior information is very tedious and time consuming. Therefore, the next and most efficient step of post acquisition data treatment is normally filtration of the list of mass spectra of interest against libraries of suspect compounds. These libraries are either home-made, from commercial supplies or from the NORMAN network and are the most critical part of the suspect screening approach.

Suspect screening leads directly to level 3 (Tentative candidates), which can be described as a "grey zone", where evidence exists for possible structure(s), but insufficient information for one exact structure only. In fortunate cases it can also lead directly to level $1 \& 2$, which means tentatively identified and maybe even semiquantified.

In this study instruments from different vendors were used and both data formats and software solutions are slightly different. As a typical example the workflow and details for analysis with LC-HR-OToF in negative ESI-mode are described in detail: Raw data acquired with data dependent acquisition mode was analyzed with Mass Hunter Qualitative analysis software (B.07). In the first step, compounds were extracted from the raw data with molecular feature extractor (MFE) algorithm. "The MFE algorithm is a compound finding technique that locates individual sample components (molecular features), even when chromatograms are complex and compounds are not well resolved. MFE locates ions that are covariant (rise and fall together in abundance) but the analysis is not exclusively based on chromatographic peak information. The algorithm uses the accuracy of the mass measurements to group related ions-related by charge-state envelope, isotopic distribution, and/or the presence of adducts and dimers. It assigns multiple species (ions) that are related to the same neutral molecule (for example, ions representing multiple charge states or adducts of the same neutral molecule) to a single compound that is referred to as a feature. Using this approach, the MFE algorithm can locate multiple compounds within a single chromatographic peak." (Sana, Roark, Li, Waddell, \& Fischer, 2008).

In the next step, elemental compositions (molecular formulas) for the unknowns based on the mass spectral data were derived and a number of suspect lists (commercial from Agilent and NILU-list) and mass spectral libraries (including Agilent PCDLs: Forensic Tox, Pesticides and Water Screening) were searched and matched with information available after treatment of raw data.

As a third step, data obtained after treatment with MFE was exported into an open accessible FOR-IDENT platform (Grosse \& Letzel, 2016), where a "molecular screening" workflow was applied and allowed to reveal additional compounds.

Compounds, which also are detected in the related blank samples, are reported only if the peak area ratio between the blank and the sample exceeded a factor of 10. 


\subsubsection{Non-target screening}

As described in the previous chapter, non-target identification in the meaning of identification of compounds without any prior information is very time consuming, and can only be done for selected and highly prioritized signals. The selection of signals for further study and evaluation is not an easy task, since nearly all sample types show a huge amount of signals, which are resulting from compounds of natural origin. Different data filtration or suspect screening methods have been applied.

Non-target screening is equivalent to level 4 and 5 (Molecular formula and exact mass). Level 4, unequivocal molecular formula can be reached, when a formula can be unambiguously assigned using the spectral information (e.g., adduct, isotope, and/or fragment information), however, there is not enough information available to propose possible structures. In level 5 , Exact mass $(\mathrm{m} / \mathrm{z}$ ) can be measured in a sample and be of specific interest for the investigation, but information lacks to assign even a molecular formula.

Non-target data evaluation according to this definition was not part of this study; however, all data are stored on an ftp-server and will be available for further data treatment in future. 



\section{Results and Discussion}

A complete list of all tentatively or fully identified compounds (Level 1-3) is given in the Appendix. All raw data containing information, which can be used for non-target analysis in future is stored on an ftp-server hosted by NILU as described in chapter 2.2.3.

\subsection{Results from target and suspect screening}

The project group has a huge number of different reference standards available and it was possible to identify and (semi)quantify: Per- and polyfluorinated compounds (PFC), chlorinated and brominated compounds, different flame retardants, bisphenols, polycyclic aromatic compounds (PAC), industrial additives like UV-stabilizers, antioxidants, and plasticizers, and pharmaceuticals and personal care products (PPCP). When identified by GC the concentration was estimated in a semi-quantitative way. However, today it is not yet possible to estimate concentrations, when using LC-MS technology.

\subsubsection{Per-and polyfluorinated compounds (PFC)}

In table 4 examples of identified PFCs are listed.

Table 4: Examples of identified per- and polyfluorinated compounds (PFC)

\begin{tabular}{|c|c|c|c|c|}
\hline Compound/Compound group & CAS & Matrix & Frequency & Country \\
\hline PFNS & $68259-12-1$ & Fish & $2 / 7$ & $\mathrm{Fl}, \mathrm{SE}$ \\
\hline PFHxPA & $355-46-4$ & Effluent & $2 / 7$ & $\mathrm{Fl}, \mathrm{SE}$ \\
\hline \multirow[t]{2}{*}{ PFHXA } & \multirow{2}{*}{$307-24-4$} & Effluent & $2 / 7$ & $\mathrm{FI}, \mathrm{DK}$ \\
\hline & & Sediment & $4 / 7$ & $\mathrm{FI}, \mathrm{SE}, \mathrm{NO}, \mathrm{FO}$ \\
\hline PFUnDA & $2058-94-8$ & Fish & $4 / 7$ & $\mathrm{Fl}, \mathrm{SE}, \mathrm{DK}, \mathrm{NO}$ \\
\hline \multirow[t]{2}{*}{ PFOS } & \multirow[t]{2}{*}{$1763-23-1$} & Fish & $6 / 7$ & $\mathrm{FI}, \mathrm{SE}, \mathrm{DK}, \mathrm{IS}, \mathrm{NO}, \mathrm{GL}$ \\
\hline & & Effluent & $7 / 7$ & $\mathrm{FI}, \mathrm{SE}, \mathrm{DK}, \mathrm{IS}, \mathrm{NO}, \mathrm{GL}, \mathrm{FO}$ \\
\hline \multirow[t]{2}{*}{ PFNA } & \multirow[t]{2}{*}{$375-95-1$} & Fish & $3 / 7$ & $\mathrm{FI}, \mathrm{SE}, \mathrm{DK}$ \\
\hline & & Effluent & $5 / 7$ & $\mathrm{FI}, \mathrm{SE}, \mathrm{DK}, \mathrm{NO}, \mathrm{FO}$ \\
\hline 4:2 FTMAC & $1799-84-4$ & Effluent & $5 / 7$ & $\mathrm{SE}$, DK, NO, GL, FO \\
\hline 4:3 Acid & $80705^{-13-1}$ & Fish & $2 / 7$ & $\mathrm{SE}, \mathrm{GL}$ \\
\hline C6/C6-PFPIA & $40143-77-9$ & Sediment & $2 / 7$ & $\mathrm{Fl}, \mathrm{SE}$ \\
\hline FOSA & $754-91-6$ & Sediment & 1/7 & NO \\
\hline 4:2 FTAL & $135984-67-7$ & Sediment & $1 / 7$ & NO \\
\hline 4:2 FTOH & $2043-47-2$ & Sediment & $1 / 7$ & NO \\
\hline
\end{tabular}

Residues of perfluorinated alkyl acids (PFAAs) were found in different matrices, and as expected both perfluoro carboxylic acids (PFCAs) and perfluoro sulfonic acids ((PFSAs) were found frequently. However, also a member of a newer class of PFAAs with a phosphonic acid as hydrophilic group, perfluorohexyl phosphonic acid (PFHxPA), was 
tentatively identified in water samples from Sweden and Finland. Perfluorinated phosphonic acids, PFPAs, are used as anti-foaming agents in the textile industry, in pesticides and lubricants (registered use in Sweden) (Keml, 2006). Recent study indicates that PFPAs likely have high persistence and long-range transport potential (Wang, Cousins, Berger, Hungerbuhler, \& Scheringer, 2016). PFHxPA has been reported in surface water from Japan (Zushi et al., 2011), Germany (Llorca et al., 2012), China (Jin, Zhang, Zhu, \& Martin, 2015), Canada (D'Eon J et al., 2009), in wastewater treatment plant effluents from Canada (D'Eon J et al., 2009) and Germany (Llorca et al., 2012)and in indoor dust from Canada (De Silva, Allard, Spencer, Webster, \& Shoeib, 2012).

In addition, emerging compounds like 4:2 FTMAC (1799-84-4) were identified in five out of seven effluent samples, 4:3 Acid (80705-13-1) in two of seven fish samples, and C6/C6-PFPIA (40143-77-9), FOSA (754-91-6), 4:2 FTAL (135984-67-7), and 4:2 FTOH (2043-47-2) were identified in one or two of the seven sediment samples.

The identified PFCs were measured by LC-MS and no information on concentration exists. It is therefore not possible to evaluate the environmental consequences of these findings. Nevertheless the identified emerging compounds should be further investigated with target methods.

\subsubsection{Chlorinated and brominated compounds}

A long range of different chlorinated and brominated compounds were frequently detected especially in sediment and fish samples, but also in some water samples as shown in table 5 .

Table 5: Examples of identified chlorinated and brominated compounds

\begin{tabular}{lllll} 
Compound/Compound group & CAS & Matrix & Frequency & Country \\
PFNS & $68259-12-1$ & Fish & $2 / 7$ & $\mathrm{Fl}, \mathrm{SE}$ \\
PFHxPA & $355-46-4$ & Effluent & $2 / 7$ & $\mathrm{Fl}, \mathrm{SE}$ \\
PFHXA & $307-24-4$ & Effluent & $2 / 7$ & $\mathrm{Fl}, \mathrm{DK}$ \\
& & Sediment & $4 / 7$ & $\mathrm{Fl}, \mathrm{SE}, \mathrm{NO}, \mathrm{FO}$ \\
PFUnDA & $2058-94-8$ & Fish & $4 / 7$ & $\mathrm{Fl}, \mathrm{SE}, \mathrm{DK}, \mathrm{NO}$ \\
PFOS & $1763-23-1$ & Fish & $6 / 7$ & $\mathrm{Fl}, \mathrm{SE}, \mathrm{DK}, \mathrm{S}, \mathrm{NO}, \mathrm{GL}$ \\
& & Effluent & $7 / 7$ & $\mathrm{Fl}, \mathrm{SE}, \mathrm{DK}, \mathrm{S}, \mathrm{NO}, \mathrm{GL}, \mathrm{FO}$ \\
PFNA & $375-95-1$ & Fish & $3 / 7$ & $\mathrm{Fl}, \mathrm{SE}, \mathrm{DK}$ \\
& & Effluent & $5 / 7$ & $\mathrm{Fl}, \mathrm{SE}, \mathrm{DK}, \mathrm{NO}, \mathrm{FO}$ \\
4:2 FTMAC & $1799-84-4$ & Effluent & $5 / 7$ & $\mathrm{SE}, \mathrm{DK}, \mathrm{NO}, \mathrm{GL}, \mathrm{FO}$ \\
4:3 Acid & $80705-13-1$ & Fish & $2 / 7$ & $\mathrm{SE}, \mathrm{GL}$ \\
C6/C6-PFPIA & $40143-77-9$ & Sediment & $2 / 7$ & $\mathrm{Fl}, \mathrm{SE}$ \\
FOSA & $754-91-6$ & Sediment & $1 / 7$ & $\mathrm{NO}$ \\
4:2 FTAL & $135984-67-7$ & Sediment & $1 / 7$ & $\mathrm{NO}$ \\
4:2 FTOH & $2043-47-2$ & Sediment & $1 / 7$ & $\mathrm{NO}$ \\
\hline
\end{tabular}

Note: ${ }^{1)}$ Also detected in the related blank samples, reported only if the threshold between the blank and the sample exceeded 10.

In some sediment and all biota samples PCB was found. The chlorinated phosphorous flame retardant TCPP was detected quite frequently, however, this compound is also detected in the blank samples and the exact environmental concentration remains uncertain. 
In the sediment sample from Sweden several chlorinated aromatic compounds were found: 3,4,5-trichlorobenzenamine (634-91-3), 1-chloro-3-isocyanatobenzene (2909-38-8), and dichloro and trichloroaniline (CAS 608-27-5 and 634-91-3). Several substituted aniline derivatives are used in the production of dyes and herbicides (Kahl et al., 2000) and these findings may be related to emissions from industrial processes.

2,4-dibromophenol, 2,4,6-tribromoanisole, and 2,4,6-Tribromophenol were detected in all fish samples (not shown in the table 5 , see Appendix tables). Bromophenoles and anisoles can be of both industrial and natural origin (Gribble, 2010). In fish samples from Greenland and Faroe Islands a mixed halogenated compound with 3 bromine and 3 chlorine was detected. The exact molecular formula was not possible to determine, however, there are indications that this compound is a natural halogenated monoterpene. Until now, nearly 5000 naturally produced halogencontaining chemicals have been found (Gribble, 2010). Mixed halogenated monoterpenes have been found in different matrices from the marine food chain. The structure of some of the compounds could be elucidated. Red algae has been identified as a major source of these compounds. Algae bloom events may explain the distribution pattern of this compound.

\subsubsection{Bisphenols}

As shown in table 6 several new bisphenols were identified in different matrices. There is a growing concern that bisphenol A (BPA) which is being used in plastics, receipts, food packaging and other products might be harmful to human health due to its actions as an endocrine-disrupting chemical (Kitamura et al., 2005). Following opinions of scientists, public and regulators manufacturers have begun to replace bisphenol A from their products with a gradual shift to using bisphenol A analogues in their products. These days two of the analogues - bisphenol S (BPS) and bisphenol F (BPF) have been mostly used as bisphenol A replacements. BPS is used in a variety of applications, for example as a developer in a thermal paper, even in the products marketed as "BPA-free paper" (Liao, Liu, \& Kannan, 2012). BPS is also used in some industrial applications like electroplating solvent and as constituent of phenolic resins (Clark, 2000). BPF is used to make epoxy resins and coatings such as tanks and pipe linings, industrial floors, adhesives, coatings and electrical varnishes (Fiege et al., 2000).

Similarly, to the $\mathrm{S}$ and $\mathrm{F}$ analogues also the other bisphenols are found in polymer materials and can be found in the samples taken in this study. 
Table 6: Examples of identified bisphenols

\begin{tabular}{lllll} 
Compound/Compound group & CAS & Matrix & Frequency & Country \\
Bisphenol A & & Sediment & $3 / 7$ & $\mathrm{FO}, \mathrm{Fl}, \mathrm{SE}$ \\
& $80-05-7$ & Effluent & $4 / 7$ & $\mathrm{Fl}, \mathrm{IS}, \mathrm{GL}, \mathrm{FO}$ \\
Bisphenol S & & Effluent & $4 / 7$ & $\mathrm{IS}, \mathrm{NO}, \mathrm{GL}, \mathrm{FO}$, \\
& $80-09-1$ & Sediment & $1 / 7$ & $\mathrm{IS}$ \\
& & Fish & $2 / 7$ & $\mathrm{GL}, \mathrm{FO}$ \\
4,4 -bisphenol F & & Effluent & $4 / 7$ & $\mathrm{Fl}, \mathrm{SE}, \mathrm{IS}, \mathrm{NO}$, \\
& \multirow{2}{*}{$620-92-8$} & Sediment & $2 / 7$ & $\mathrm{NO}, \mathrm{FO}$, \\
Bisphenol E & & Effluent & $1 / 7$ & $\mathrm{DK}$ \\
Bisphenol AF & $2081-08-5$ & Effluent & $2 / 7$ & $\mathrm{GL}$, \\
& $1478-61-1$ & Fish & $4 / 7$ & $\mathrm{IS}, \mathrm{NO}, \mathrm{GL}, \mathrm{DK}$ \\
& & Sediment & $1 / 7$ & $\mathrm{Fl}$ \\
\hline
\end{tabular}

\subsubsection{Polycyclic aromatic compounds (PAC)}

The most prominent compound group identified in sediments are polycyclic aromatic compounds, both the pure hydrocarbons (PAHs: pyrene, phenanthrene, perylene etc.), but also heteroaromatics as benzothiazoles, dibenzofuran, acridine, and thiopenes are found. PACs are both of natural and anthropogenic origin. Typical processes which form PACs, are all forms of incomplete combustion, pyrolysis, and geological transformation of organic material. Prominent sources are smoke, soot, tar, creosote, coal, and other fossil products. PACs are often characterized as unintentional by-products.

Table 7: Examples of identified PACs

\begin{tabular}{lllll} 
Compound/Compound group & CAS & Matrix & Frequency & Country \\
9-methyl acridine & $611-64-3$ & Effluent & $5 / 7$ & $\mathrm{Fl}, \mathrm{SE}, \mathrm{DK}, \mathrm{NO}, \mathrm{GL}$, \\
Fluoranthene & $206-44-0$ & Sediment & $6 / 7$ & $\mathrm{Fl}, \mathrm{SE}, \mathrm{DK}, \mathrm{NO}, \mathrm{GL}, \mathrm{FO}$ \\
Pyrene & $129-00-0$ & Sediment & $6 / 7$ & $\mathrm{Fl}, \mathrm{SE}, \mathrm{DK}, \mathrm{IS}, \mathrm{NO}, \mathrm{FO}$ \\
Benz[b]fluoranthene & $205-99-2$ & Sediment & $4 / 7$ & $\mathrm{FO}, \mathrm{Fl}, \mathrm{SE}, \mathrm{DK}$ \\
Benzothiazole & $95-16-9$ & Sediment & $6 / 7$ & $\mathrm{Fl}, \mathrm{GL}, \mathrm{IS}, \mathrm{DK}, \mathrm{SE}, \mathrm{FO}$ \\
\hline
\end{tabular}

\subsubsection{Other industrial additives like UV-stabilizers, antioxidants and plasticizers}

Several industrial additives were found in sediment, water and fish samples. These were for instance phenols, UV-stabilizers, antioxidants, and plasticizers of phthalate-type. Also a benzothiazole compound (2-methylthiobenzothiazole, MTBT, CAS: 615-22-5) was frequently found in all sample types and in high concentrations. 2Methylthiobenzothiazole is a degradation product of mercaptobenzothiazol (MBT, CAS: 149-30-4), which is used in vulcanization of rubber. MTBT has earlier been detected in other screening studies (Blum et al., 2017) in water and other abiotic samples, however, to our best knowledge not yet in marine biota. 
Table 8: Examples of identified industrial additives

\begin{tabular}{lllll}
\hline Compound/Compound group & CAS & Matrix & Frequency & Country \\
2-(methylthio)benzothiazole & $615-22-5$ & Water & $5 / 7$ & $\mathrm{Fl}, \mathrm{SE}, \mathrm{DK}, \mathrm{IS}, \mathrm{FO}$ \\
& & Sediment & $2 / 7$ & $\mathrm{Fl}, \mathrm{NO}$ \\
& & Biota & $3 / 7$ & $\mathrm{SE}, \mathrm{DK}, \mathrm{NO}$
\end{tabular}

\subsubsection{Pharmaceuticals and Personal care products (PPCPs)}

By suspect screening a huge number of pharmaceuticals were identified in effluent samples. Many of the identified pharmaceuticals are not only found in effluents from sewage treatment plants (STPs) with basic or no treatment but also in STPs with advanced treatment technologies.

Different personal care products were frequently detected in all sample types. Some selected examples are shown in table 9 . Triacetin is used as food additive and as a humectant (attracting moisture) in different applications, especially pharmaceuticals. Paroxypropione (70-70-2), a non-steroidal xeno-estrogen, was found in 5 of 7 fish samples. Paroxypropione has structural similarity to parabens, diethylstilbestrol, and alkylphenols, which all are recognized as xeno-estrogens. Methyl salicylate is a salicylic acid derivative, naturally produced by many species of plants, although nowadays it is industrially produced by esterification of the acid with methanol. At high concentrations, this compound acts as a rubefacient, analgesic, and anti-inflammatory. In low concentrations it is also used as a flavoring agent in chewing gums and mints. Its antimicrobial properties are also used in antiseptic mouthwash. Different UV filters (benzophenones and PBS) were found in sediment and effluents. Galaxolide, a musk compound used in high volumes for example by perfume and cologne manufacturers has frequently been found in effluent and fish samples.

Carvone is a terpenoid that has been frequently found in effluents. The compound itself is found naturally in many essential oils, but is most abundant in the oils from seeds of caraway (Carum carvi), spearmint (Mentha spicata), and dill (de Carvalho \& da Fonseca, 2006). Spearmint gums are major uses of natural spearmint oil, and oils containing carvones are used for air freshening products and in aromatherapy. 
Table 9: Examples of identified personal care products

\begin{tabular}{|c|c|c|c|c|}
\hline Compound/Compound group & CAS & Matrix & Frequency & Country \\
\hline \multirow[t]{2}{*}{ Triacetin } & $102-76-1$ & Effluent & $3 / 7$ & $\mathrm{SE}, \mathrm{IS}, \mathrm{NO}$ \\
\hline & & Sediment & $2 / 7$ & $\mathrm{SE}, \mathrm{FO}$, \\
\hline \multirow[t]{2}{*}{ Methyl salicylate } & $119-36-8$ & Fish & $1 / 7^{1)}$ & SE \\
\hline & & Effluent & $1 / 7^{1)}$ & $\mathrm{SE}, \mathrm{IS}$ \\
\hline Benzophenone-1 & $119-61-9$ & sediment & $1 / 7^{1)}$ & $\mathrm{GL}$, \\
\hline Benzophenone-4 & $4065-45-6$ & Effluent & $4 / 7$ & $\mathrm{FI}, \mathrm{SE}, \mathrm{DK}, \mathrm{NO}$ \\
\hline Phenylbenzimidazole sulfonic acid (PBS) & & Effluent & $5 / 7$ & $\mathrm{FI}, \mathrm{SE}, \mathrm{DK}, \mathrm{NO}, \mathrm{FO}$ \\
\hline Paroxypropione & $70-70-2$ & Fish & $5 / 7$ & $\mathrm{FI}, \mathrm{SE}, \mathrm{DK}, \mathrm{GL}, \mathrm{FO}$ \\
\hline \multirow[t]{2}{*}{ Galaxolide } & $1222-05-5$ & Effluent & $6 / 7$ & $\mathrm{FI}, \mathrm{SE}, \mathrm{DK}, \mathrm{NO}, \mathrm{GL}, \mathrm{FO}$, \\
\hline & & Fish & $4 / 7$ & $\mathrm{SE}, \mathrm{IS}, \mathrm{NO}, \mathrm{FO}$ \\
\hline Carvone & $99-49-0$ & Effluent & $5 / 7$ & $\mathrm{FI}, \mathrm{SE}, \mathrm{IS}, \mathrm{GL}, \mathrm{FO}$ \\
\hline
\end{tabular}

Note: ${ }^{1)}$ Also detected in the related blank samples, reported only if the threshold between the blank and the sample exceeded 10 .

\subsection{Comparison of effluent water concentrations}

The effluent water samples are similar enough to allow a comparison of contaminant levels. Table 10 summarizes the results of the effluent analyses of the most frequently detected compounds (found in at least three effluents). Because the measurements were semi-quantitative in nature, the table only include the maximum concentration of each contaminant (and that value should be regarded as indications only). The focus of the comparison is on the relative concentrations between sewage treatment plants (STP), which should be more reliable.

The STPs are ordered according to the total concentration detected. It is clear that the levels in the effluent is higher for the STPs with less advanced sewage treatment, as in FO, IS and GL. In most cases the highest contaminant concentration was found in effluent from IS or GL. However, for a number of contaminants like m-Cresol, 2-(Methylthio)pyridine, 2-(Methylthio)benzothiazole, 9-methylacridine, 3,3-Diphenylacrylonitrile, 3,3-Diphenylpropionitrile, N-butylbenzenesulfonamide, and Diclofenac the levels were highest in effluent from one of the STPs in SE, DK or FI, likely because of higher load from industry, traffic and other urban sources.

The concentrations in effluents from STPs with advanced sewage treatment (SE, $\mathrm{DK}, \mathrm{FI}, \mathrm{NO})$ vs. STPs with basic or no treatment $(\mathrm{FO}, \mathrm{IS}, \mathrm{GL})$ is likely to reflect the relative persistency of relatively water soluble contaminants. The terpenoids coumarin, carvone, menthol and tetrahydralinalol and the alkaloid caffeine are, for instance, much less abundant in effluent from STPs with advanced treatment. It is plausible that those are degraded in the activated sludge process. Caffeine is known to be easily degraded in active sludge processes (ca $99 \%$ removal). 
Table 10: Comparison of effluent concentrations of frequently detected compounds ( $n: 3-7$ ). The individual concentrations have been normalized to $100 \%$, i.e. to the sample with the highest (max) concentration (grey background), and given as relative (percent) concentrations

\begin{tabular}{|c|c|c|c|c|c|c|c|c|c|c|}
\hline Name & CAS & $\begin{array}{r}\operatorname{Max} \\
(\mu g / L)\end{array}$ & SE \% & DK \% & FI \% & NO \% & FO \% & IS \% & GL \% & Potential source(s) \\
\hline $\mathrm{m}$-Cresol & $108-39-4$ & 0.2 & 100 & - & - & 24 & - & - & 94 & Misc. \\
\hline N-Formylmorpholine & $4394-85-8$ & 0.06 & 17 & - & 39 & - & - & 100 & - & Misc. \\
\hline 2-(Methylthio)pyridine & $18438-38-5$ & 0.06 & 33 & 100 & 14 & 66 & - & - & - & Misc. \\
\hline 2-Hydroxy acetophenone & $582-24-1$ & 30 & 0.2 & 0.3 & 0.4 & 1 & 0.3 & 100 & 0.1 & Misc. \\
\hline 2-Phenoxyethanol & $122-99-6$ & 50 & 0.3 & 0.2 & 0.2 & - & 12 & 12 & 100 & Misc. \\
\hline Coumarin & $91-64-5$ & 0.08 & - & - & - & - & 30 & 100 & 85 & Fragrance, flavour \\
\hline Carvone & $99-49-0$ & 0.3 & 13 & - & 16 & - & 100 & 62 & 55 & Fragrance, flavour \\
\hline Menthol & $15356-70-4$ & 6 & - & - & - & - & 47 & 54 & 100 & Fragrance, flavour \\
\hline Tetrahydralinalol & $78-69-3$ & 4 & - & - & - & - & 26 & 25 & 100 & Fragrance, flavour \\
\hline Dimethyl adipate & $627-93-0$ & 0.2 & 13 & - & 12 & 31 & - & 100 & 29 & Plasticizer \\
\hline $\begin{array}{l}\text { 2-(Methylthio) } \\
\text { benzothiazole }\end{array}$ & $615-22-5$ & 0.2 & 53 & 76 & 100 & - & 22 & 53 & - & Rubber \\
\hline Benzophenone & $119-61-9$ & 0.4 & 26 & 25 & 33 & 61 & 18 & 78 & 100 & PPCP \\
\hline $\begin{array}{l}\text { Dipropylene glycol, butyl } \\
\text { ether }\end{array}$ & 29911-28-2 & 4 & 0.4 & - & - & 62 & 5 & 100 & 24 & Insecticide, solvent \\
\hline 9-methylacridine & $611-64-3$ & 1 & 18 & 30 & 100 & 18 & - & - & 74 & Dyes \\
\hline Caffeine & $58-08-2$ & 30 & 0.2 & 0.2 & 0.2 & 2 & 9 & 99 & 100 & Coffee, pharma \\
\hline $\begin{array}{l}\text { 4-Chloro-2-methyl-1-phenyl- } \\
\text { 3-Buten-1-ol }\end{array}$ & - & 0.1 & 26 & - & 35 & - & - & - & 100 & Unknown \\
\hline $\begin{array}{l}\text { 2-Methyl-1- } \\
\text { Benzyloxybenzene }\end{array}$ & $19578-70-2$ & 7 & 1 & - & 2 & 2 & - & 100 & 3 & Unknown \\
\hline 3,3-Diphenylacrylonitrile & $3531-24-6$ & 0.04 & - & 100 & 32 & 61 & - & - & - & Org. synthesis \\
\hline 3,3-Diphenylpropionitrile & $2286-54-6$ & 1 & 22 & 100 & 40 & 6 & - & 6 & - & Org. synthesis \\
\hline $\begin{array}{l}\mathrm{N} \text {-butyl- } \\
\text { benzenesulfonamide }\end{array}$ & $3622-84-2$ & 0.1 & - & 100 & - & 38 & - & - & 53 & Plasticizer \\
\hline Triacetin & $102-76-1$ & 0.2 & 31 & - & 32 & 50 & - & 100 & - & Plasticizer \\
\hline Galaxolide & $1222-05-5$ & 3 & 13 & 8 & 8 & 13 & 9 & - & 100 & Fragrance, flavour \\
\hline Undecyl benzoate & $6316-30-9$ & 1 & 7 & 4 & 5 & 8 & 5 & 21 & 100 & PPCP \\
\hline Diclofenac & $15362-40-0$ & 0.2 & 46 & 31 & 100 & - & - & - & - & Pharma \\
\hline Myristyl benzoate & $68411-27-8$ & 2 & 5 & 4 & 5 & 6 & 4 & 20 & 100 & PPCP \\
\hline TCPP & $13674-84-5$ & 1 & 17 & 23 & 44 & - & - & - & 100 & Flame retardant \\
\hline Pentadecyl benzoate & $68411-27-8$ & 0.7 & 9 & 6 & 0 & 11 & 5 & 29 & 100 & PPCP \\
\hline
\end{tabular}





\section{Conclusions}

With an initial suspect data treatment using different mass spectral data bases it was possible to detect and identify a surprisingly long list of emerging compounds with a level of confidence of three or better. This list is including per- and polyfluorinated compounds (PFC), chlorinated and brominated compounds, flame retardants, bisphenols, polycyclic aromatic compounds (PAC), industrial additives, and pharmaceuticals and personal care products (PPCPs).

When evaluating the total outcome of this study, it is important to keep the following limitations in mind: in order to get a wide overview over all anthropogenic compounds in environmental samples, a general sample preparation method has to be chosen. Many compounds, which easily could be detected by a dedicated target method, will be masked or lost either by (1) interfering matrix, (2) instrumental overload/saturation, and (3) possible loss of compounds. Furthermore, the applied GC/LC methods are often not optimal for each single analyte. For the time being, suspect/non-target cannot replace dedicated target methods in sensitivity and specificity, but proofs to be an important tool for identification of compounds of emerging concern.

Non-target identification in the meaning of identification of compounds without any prior information was not part of this study. However, raw data will be stored and made available for retrospective studies. New data treatment tools for real non-target work are under development among several members of the NORMAN network. It has become possible to identify new compounds due to gradients as for instance (1) increasing dilution with growing distance from sources (spatial gradient) (Alygizakis, Oswald, Thomaidis, \& Slobodnik, 2016) or (2) increasing bioaccumulation throughout a food chain (Thomas et al., 2015). The project group strongly recommend taking advantage of the extensive knowledge of the NORMAN network and the NORMAN Working group on Non-target screening especially in the phase of selecting sample types and locations. 



\section{Acknowledgements}

\subsection{Denmark}

Thanks to Anna Gade Holm and Lisbeth Nielsen, Danish Environmental Agency for assistance with selection and collection of samples of effluent and sludge from the waste water treatment plants. Thanks to Jakob Strand and Martin M. Larsen, Institut for Bioscience, Aarhus University for assistance with selection and collection of samples of sediment and fish from marine areas.

\subsection{Faroe Islands}

The sampling in Álakeri, at the outlet of the Main Hospital sewage treatment plant, demanded some extraordinary sampling crew, and the assistance of the professional divers students at FaroeDive diving school in September 2015 are gratefully acknowledged. So is the assistance of the crew at the SAR vessel Liv who took part in the not so successful fishery expedition, and my colleague at the Environment Agency, Suni Petersen who assisted in a fisheries excursion on his boat himself as skipper. Thanks also to Høgni Arnbjarnarson for lending his fishery gear. Thanks are due also to my colleagues Rakul Mortensen and Jóhannis Danielsen who successfully landed a number of coalfish.

\subsection{Finland}

Thanks to Markku Korhonen, Finnish Environment Institute (SYKE) for coordinating sampling logistics and personnel at the city of Helsinki for providing the samples for this project.

\subsection{Greenland}

All Greenlandic samples were collected by Greenland Institute of Natural Resources.

\subsection{Iceland}

Thanks to Halldór Pálmar Halldórsson from The Sudurnes research center of the University of Iceland for assisting with taking samples to this project. 


\subsection{Norway}

Thanks to Sigurd $\varnothing x$ nevad and Kevin Thomas from The Norwegian Institute for Water Research (NIVA) for delivering the Norwegian samples to this project.

\subsection{Sweden}

The collection of Swedish samples was coordinated by IVL Swedish Environmental Research Institute. 


\section{References}

Alygizakis, N. A., Oswald, P., Thomaidis, N. S., \& Slobodnik, J. (2016). Exploring the Extent of Chemical Polltuion Transfer from the Danube River to Black Sea.

Blum, K. M., Andersson, P. L., Renman, G., Ahrens, L., Gros, M., Wiberg, K., \& Haglund, P. (2017). Non-target screening and prioritization of potentially persistent, bioaccumulating and toxic domestic wastewater contaminants and their removal in on-site and large-scale sewage treatment plants. Sci Total Environ, 575, 265-275.

https://doi.org/10.1016/j.scitotenv.2016.09.135

Clark, E. (2000). Sulfolane and Sulfones Kirk-Othmer Encyclopedia of Chemical Technology: John Wiley \& Sons, Inc.

D'Eon J, C., Crozier, P. W., Furdui, V. I., Reiner, E. J., Libelo, E. L., \& Mabury, S. A. (2009). Perfluorinated phosphonic acids in Canadian surface waters and wastewater treatment plant effluent: discovery of a new class of perfluorinated acids. Environ Toxicol Chem, 28(10), 21012107. https://doi.org/10.1897/og-048.1

de Carvalho, C. C. C. R., \& da Fonseca, M. M. R. (2006). Carvone: Why and how should one bother to produce this terpene. Food Chemistry, 95(3), 413-422.

https://doi.org/10.1016/j.foodchem.2005.01.003

De Silva, A. O., Allard, C. N., Spencer, C., Webster, G. M., \& Shoeib, M. (2012). Phosphoruscontaining fluorinated organics: polyfluoroalkyl phosphoric acid diesters (diPAPs), perfluorophosphonates (PFPAs), and perfluorophosphinates (PFPIAs) in residential indoor dust. Environ Sci Technol, 46(22), 12575-12582. https://doi.org/10.1021/es303172p

Fiege, H., Voges, H.-W., Hamamoto, T., Umemura, S., Iwata, T., Miki, H. Paulus, W. (2000). Phenol Derivatives Ullmann's Encyclopedia of Industrial Chemistry: Wiley-VCH Verlag GmbH \& Co. KGaA.

Gribble, G. W. (2010). Naturally Occurring Organohalogen Compounds - A Comprehensive Update. Wien: Springer-Verlag. https://doi.org/10.1007/978-3-211-99323-1

Grosse, S., \& Letzel, T. (2016). User Manual for FOR-IDENT Database (pp. 29).

Jin, H., Zhang, Y., Zhu, L., \& Martin, J. W. (2015). Isomer profiles of perfluoroalkyl substances in water and soil surrounding a chinese fluorochemical manufacturing park. Environ Sci Technol, 49(8), 4946-4954. https://doi.org/10.1021/acs.est.5boo212

Kahl, T., Schröder, K.-W., Lawrence, F. R., Marshall, W. J., Höke, H., \& Jäckh, R. (2000). Aniline Ullmann's Encyclopedia of Industrial Chemistry: Wiley-VCH Verlag GmbH \& Co. KGaA.

Kaj, L., Wallberg, P., \& Brorström-Lundén, E. (2014). Quaternary ammonium compounds Analyses in a Nordic cooperation on screening. Retrieved from Copenhagen:

Keml, S. C. A. (2006). Perfluorinated substances and their uses in Sweden. Retrieved from

Kitamura, S., Suzuki, T., Sanoh, S., Kohta, R., Jinno, N., Sugihara, K. Ohta, S. (2005). Comparative study of the endocrine-disrupting activity of bisphenol $A$ and 19 related compounds. Toxicol Sci, 84(2), 249-259. https://doi.org/10.1093/toxsci/kfio74

Liao, C., Liu, F., \& Kannan, K. (2012). Bisphenol s, a new bisphenol analogue, in paper products and currency bills and its association with bisphenol a residues. Environ Sci Technol, 46(12), 6515-6522. https://doi.org/10.1021/es300876n

Llorca, M., Farre, M., Pico, Y., Muller, J., Knepper, T. P., \& Barcelo, D. (2012). Analysis of perfluoroalkyl substances in waters from Germany and Spain. Science of The Total Environment, 431, 139-150. https://doi.org/10.1016/j.scitotenv.2012.05.011

Sana, T. R., Roark, J. C., Li, X., Waddell, K., \& Fischer, S. M. (2008). Molecular formula and METLIN Personal Metabolite Database matching applied to the identification of compounds generated by LC/TOF-MS. J Biomol Tech, 19(4), 258-266. 
Schymanski, E. L., Jeon, J., Gulde, R., Fenner, K., Ruff, M., Singer, H. P., \& Hollender, J. (2014). Identifying Small Molecules via High Resolution Mass Spectrometry: Communicating Confidence. Environmental Science \& Technology, 48(4), 2097-2098.

https://doi.org/10.1021/es5002105

Schymanski, E. L., Singer, H. P., Slobodnik, J., Ipolyi, I. M., Oswald, P., Krauss, M. Hollender, J. (2015). Non-target screening with high resolution mass spectrometry: Critical review using a collaborative trial on water analysis. Anal Bioanal Chem, (accepted).

https://doi.org/10.1007/s00216-015-8681-7

Thomas, K. V., Schlabach, M., Langford, K., Reid, M., Fjeld, E., Øxnevad, S. Borgen, A. (2015). Screening programme 2014: Phosphites, selected PBT substances and non-target screening (33/2015). Retrieved from Oslo/Kjeller:

Wang, Z., Cousins, I. T., Berger, U., Hungerbuhler, K., \& Scheringer, M. (2016). Comparative assessment of the environmental hazards of and exposure to perfluoroalkyl phosphonic and phosphinic acids (PFPAs and PFPiAs): Current knowledge, gaps, challenges and research needs. Environ Int, 89-90, 235-247. https://doi.org/10.1016/j.envint.2016.01.023

Zushi, Y., Ye, F., Motegi, M., Nojiri, K., Hosono, S., Suzuki, T. Masunaga, S. (2011). Spatially detailed survey on pollution by multiple perfluorinated compounds in the Tokyo Bay basin of Japan. Environ Sci Technol, 45(7), 2887-2893. https://doi.org/10.1021/es103917r 


\section{Sammendrag}

På oppdrag fra Nordisk Ministerråd representert av Nordisk kjemikaliegruppe og Havgruppen (HAV) gjennomførte NILU-Norsk Institutt for Luftforskning, Norsk Institutt for Vannforskning (NIVA) og Universitet Umeå-Kemiska institutionen en suspect screening studie som omfattet utløpsvann fra renseanlegg, sediment og fiskeprøver fra alle sju nordiske land.

Denne studien var opprinnelig planlagt som non-target screening, men fagfeltet er under rivende utvikling og stadig nye databearbeidingsrutiner blir utviklet. Dette medfører at man nå skiller strengere mellom de forskjellige konseptene og «suspect screening» har kommet opp som et nytt begrep eller konsept. Det tidligere begrepet non target screening er nå erstattet av suspect screening som baserer seg på omfattende bruk av MS-biblioteker, mens non-target screening kun brukes for dataevaluering av stoffer som man ikke finner i biblioteker.

Hvert av de deltakende land var selv ansvarlig for prøveutvalg, innsamling og forsendelse til analyselaboratoriene som fulgte et på forhånd utarbeidet felles prøvetakingsprotokoll. I hvert land ble det tatt en prøve av utløpsvann fra et stort kommunalt avløpsverk, en sedimentprøve og en fiskeprøve. Vann og sedimentprøver ble tatt som stikkprøver, mens fiskeprøvene var samleprøver av flere individer av samme art. Med unntak av i Danmark ble alle sediment og fiskeprøver tatt i nærhet av vannutløpet. Alle innsamlete prøver ble sent til NIVA, Oslo, Norge for lagring og opparbeidelse. For ikke å fjerne potensielt interessante stoffer ble det valgt to veldig generelle og omfattende ekstraskjons- og opparbeidelsesprosedyrer. Den første var tilpasset og optimalisert for ikke-polare og veldig lipofile forbindelser som PCB, PAH og andre klassiske POPer, mens den andre var optimalisert for mer polare komponenter slik som medisiner, moderne pesticider og biocider, PFAS og bisfenoler. Før starten ble det tilsatt et utvalg isotopmarkerte standarder for å kontrollere opparbeidelsen og for bruk i kvantifiseringen. De ferdige ekstraktene ble sendt til de forskjellige laboratorier i prosjektgruppen.

For å dekke et størst mulig spenn av forskjellige stoffer ble både GC- og LC-MS teknikker brukt (1. GCxGC-HRMS, 2. GC-ECNI-HRMS, 3. LC-OToFMS ESI+, and 4. LCQToFMS ESI-). Rådata fra alle analyser i prosjektet er lagret på NILUs FTP-server, og er tilgjengelig også for andre forskere i feltet. Som første trinn i databearbeidingen ble de opprinnelige høyoppløste massespektrometriske signaler redusert til retensjonstid, centroid posisjon, areal av signalet og bredde ved halv høyde. Neste og viktigste trinn i suspect screening er en filtrering av alle signaler mot en eller flere databaser med lagrete massespektra av potensielt interessante forbindelser (suspect list). Disse databasene er både laget av instituttene selv, anskaffet fra kommersielle aktører og delt via NORMAN-nettverket. Kvalitet og innhold i databasene er avgjørende for utfallet av dette trinnet. Suspect screening prosedyren leder direkte til en liste av 
potensielle kandidatstoffer hvor man har gode indikasjoner, men ikke endelig sikkerhet for den eksakte strukturen.

Følgende stoffgrupper var det mulig å identifisere og delvis også kvantifisere: Perog polyfluorerte stoffer (PFC), diverse klorerte og bromerte forbindelser, forskjellige flammehemmere, bisfenoler, polysykliske aromatiske forbindelser (PAC), industrielle additiver som UV-stabilisatorer, antioksidanter og mykgjørere, samt legemidler og personlige pleieprodukter (PPCP). For stoffer som ble identifisert ved GC-MS var det mulig å estimere en omtrentlig konsentrasjon. Når det gjelder LC-MS teknikk, er dette foreløpig ikke mulig,

Følgende stoffer kan være relevant for en videre oppfølging:

- I tillegg til mange kjente PFAS forbindelser ble følgende «nye» forbindelser påvist i sedimentprøver: 4:2 FTMAC (1799-84-4), 4:3 Acid (80705-13-1), C6/C6-PFPIA (40143-77-9), FOSA (754-91-6), 4:2 FTAL (135984-67-7), og 4:2 FTOH (2043-47-2).

I den svenske sedimentprøven ble de funnet flere klorerte aromatiske forbindelser: 3,4,5-triklorbenzenamin (634-91-3), 1-kloro-3-isocyanatobenzen (2909-38-8), samt diklor og trikloranilin (CAS 608-27-5 and 634-91-3). Anilinderivater blir brukt $i$ produksjon av fargestoffer og herbicider og kan stamme fra utslipp ved industriell produksjon av disse stoffene, men det kan heller ikke utelukkes at det er nedbrytningsprodukter av disse stoffene.

Det ble påvist flere nye bisfenoler. Det er grunn til bekymring at blant annet bisfenol S, E og AF er påvist i fiskeprøver.

Noen industrielle additiver som fenoler, UV-stabilisatorer, antioksidanter og mykgjørere ble funnet i alle prøvetypene. En grunn til bekymring er funnet av 2metyltiobenzotiazol (MTBT, CAS: 615-22-5) i fiskeprøvene. Stoffet som brukes i vulkanisering av gummi, er ved tidligere screening studier påvist i utslippsvann og sediment, men dette er, så vidt vi vet, første funnet i biologiske prøver.

lkke veldig overraskende ble det funnet veldig mange legemidler i prøver av avløpsvann. Men det er grunn til bekymring at mange av legemidlene ikke bare finnes i avløp fra avløpsanlegg med begrenset rensing, men også i avløp fra anlegg med avansert renseteknologi. En sammenligning av kontaminering av avløp fra avanserte renseanlegg ( $\mathrm{SE}, \mathrm{DK}, \mathrm{Fl}, \mathrm{NO}$ ) og anlegg med enkel/ikke rensing (FO, IS, GL) gir informasjon om stoffenes relative persistens. For eksempel finns det mye mindre av terpenoidene kumarin, karvon, mentol og tetrahydrolinalol samt alkaloiden koffein og det antas at disse stoffene nedbrytes i den aktiverte slamprosessen. På den andre siden viser stoffer som m-kresol, 2-(metyltio)pyridin, 2-(metyltio)benzotiazol, 9-metylakridin, 3,3'difenylakrylonitril, 3,3'-difenylpropionitril, N-butyl-benzensulfonamid, og diclofenac en mye høyere konsentrasjon i avløpsprøver fra SE, DK og Fl, trolig på grunn av mye høyere tilførsel fra industri, trafikk og andre urbane kilder.

Med en innledende «suspect» databehandling som bruker forskjellige massespektroskopiske databanker, var det mulig å identifisere en forbausende lang liste av nye miljøgifter med et konfidensnivå på tre eller bedre. Når man vurderer hele resultatet av denne studien er det viktig å ta hensyn til disse begrensningene: for å få en 
bredest mulig oversikt over alle antropogene stoffer i miljøprøvene, valgte man en veldig lite spesifikk prøveforberedelse (ekstraksjon og opparbeidelse). Mange stoffer som ellers er lett å detektere med en «target» metode er enten skjult eller blitt borte på grunn av (1) interfererende matriks, (2) overbelastning eller metning av analyseinstrumentet og/eller (3) tap av stoffene under opparbeidelse. Per i dag kan «suspect/non-target screening» ikke erstatte en «target» analyse verken når det gjelder følsomhet eller spesifisitet, men «suspect/non-target screening» er uten tvil blitt et av de viktigste verktøy for identifikasjon av stoffer som kan gi anledning til bekymring for helse eller miljø. 



\section{Appendix}

Table 11: Effluent Finland

\begin{tabular}{|c|c|c|c|c|c|}
\hline $\begin{array}{l}\text { Quant } \\
\text { (ng/L) }\end{array}$ & $\begin{array}{l}\text { Molecular } \\
\text { Formula }\end{array}$ & CAS & Common name & $\begin{array}{l}\text { Occur/ } \\
\text { matrix }\end{array}$ & $\begin{array}{r}\text { ID } \\
\text { Level }\end{array}$ \\
\hline 2000,23 & $\mathrm{C}_{19} \mathrm{H}_{4} \mathrm{OO}_{2} \mathrm{Si}$ & $55520-89-3$ & Hexadecanoic acid, trimethylsilyl ester & 7 & 2 \\
\hline 1203,17 & $\mathrm{C}_{21} \mathrm{H}_{44} \mathrm{O}_{2} \mathrm{Si}$ & 18748-91-9 & Octadecanoic acid, trimethylsilyl ester & 4 & 2 \\
\hline 1058,63 & $\mathrm{C}_{14} \mathrm{H}_{11} \mathrm{~N}$ & $611-64-3$ & Acridine, 9-methyl- & 5 & 2 \\
\hline 703,39 & $\mathrm{C}_{18} \mathrm{H}_{39} \mathrm{O}_{7} \mathrm{P}$ & $78-51-3$ & Ethanol, 2-butoxy-, phosphate (3:1) & 2 & 2 \\
\hline 513,86 & $\mathrm{C}_{9} \mathrm{H}_{1} 8 \mathrm{Cl}_{3} \mathrm{O}_{4} \mathrm{P}$ & $13674-84-5$ & 2-Propanol, 1-chloro-, phosphate (3:1) & 4 & 2 \\
\hline 508,11 & $\mathrm{C}_{15} \mathrm{H}_{13} \mathrm{~N}$ & $2286-54-6$ & Benzenepropanenitrile, á-phenyl- & 5 & 2 \\
\hline 264,64 & $\mathrm{C}_{7} \mathrm{H}_{9} \mathrm{NO}_{2}$ & $20189-42-8$ & 1H-Pyrrole-2,5-dione, 3-ethyl-4-methyl- & 7 & 3 \\
\hline 212,42 & $\mathrm{C}_{1} 8 \mathrm{H}_{2} 6 \mathrm{O}$ & $1222-05-5$ & Galaxolide & 6 & 2 \\
\hline 192,61 & $\mathrm{C}_{8} \mathrm{H}_{7} \mathrm{NS}_{2}$ & $615-22-5$ & Benzothiazole, 2-(methylthio)- & 5 & 2 \\
\hline 189,64 & $\mathrm{C}_{14} \mathrm{H}_{9} \mathrm{Cl}_{2} \mathrm{NO}$ & $15362-40-0$ & $2 \mathrm{H}$-Indol-2-one, 1-(2,6-dichlorophenyl)-1,3-dihydro- & 3 & 3 \\
\hline 136,53 & $\mathrm{C} 8 \mathrm{H}_{6} \mathrm{O}_{2}$ & $1074-12-0$ & Phenylglyoxal & 6 & 2 \\
\hline 131,81 & $\mathrm{C}_{12} \mathrm{H}_{10 O}$ & $93-08-3$ & 2-Naphthyl methyl ketone & 1 & 2 \\
\hline 130,53 & $\mathrm{C}_{13} \mathrm{H}_{10 O}$ & $119-61-9$ & Benzophenone & 7 & 2 \\
\hline 129,48 & $\mathrm{C}_{8} \mathrm{H}_{10 O}$ & $122-99-6$ & Ethanol, 2-phenoxy- & 6 & 2 \\
\hline 127,05 & $\mathrm{C} 8 \mathrm{H}_{8} \mathrm{O}_{2}$ & $582-24-1$ & Ethanone, 2-hydroxy-1-phenyl- & 7 & 2 \\
\hline 104,95 & $\mathrm{C}_{14} \mathrm{H}_{14} \mathrm{O}$ & $19578-70-2$ & Benzene, 1-methyl-2-(phenylmethoxy)- & 5 & 2 \\
\hline 101,37 & $\mathrm{C}_{12} \mathrm{H}_{24} \mathrm{O}_{3}$ & $74367-34-3$ & Propanoic acid, 2-methyl-, 3-hydroxy-2,4,4-trimethylpentyl ester & 7 & 2 \\
\hline 88,82 & $\mathrm{C}_{1} \mathrm{OH}_{16}$ & $3760-14-3$ & 1,5-Cyclooctadiene, 1,5-dimethyl- & 7 & 3 \\
\hline 83,84 & $\mathrm{C}_{2} \mathrm{OH}_{32} \mathrm{O}_{2}$ & - & Benzoic acid, tridecyl ester & 7 & 3 \\
\hline 73,23 & $\mathrm{C}_{9} \mathrm{H}_{14} \mathrm{O} 6$ & $102-76-1$ & Triacetin & 4 & 2 \\
\hline 68,99 & $\mathrm{C}_{12} \mathrm{H}_{12} \mathrm{O}$ & $7228-47-9$ & à-Methyl-2-naphthalenemethanol & 1 & 2 \\
\hline 59,83 & $\mathrm{C}_{1} 8 \mathrm{H}_{2} 8 \mathrm{O}_{2}$ & $6316-30-9$ & Benzoic acid, undecyl ester & 7 & 3 \\
\hline 47,36 & $\mathrm{C}_{8} \mathrm{H}_{10} \mathrm{~N}_{4} \mathrm{O}_{2}$ & $58-08-2$ & Caffeine & 7 & 3 \\
\hline 44,51 & $\mathrm{C}_{1} 8 \mathrm{H}_{3} 8 \mathrm{O}_{2} \mathrm{Si}$ & $74367-22-9$ & n-Pentadecanoic acid, trimethylsilyl ester & 4 & 2 \\
\hline 43,64 & $\mathrm{C}_{11} \mathrm{H}_{13} \mathrm{ClO}$ & - & 3-Buten-1-ol, 4-chloro-2-methyl-1-phenyl- & 3 & 2 \\
\hline 43,08 & $\mathrm{C}_{10 \mathrm{H}_{4}} \mathrm{O}$ & $99-49-0$ & Carvone & 5 & 2 \\
\hline 24,10 & $\mathrm{C}_{9} \mathrm{H}_{12} \mathrm{O}_{2}$ & $1125-21-9$ & $2,6,6$-Trimethyl-2-cyclohexene-1,4-dione & 1 & 2 \\
\hline 23,04 & $\mathrm{C}_{5} \mathrm{H}_{9} \mathrm{NO}_{2}$ & $4394-85-8$ & N-Formylmorpholine & 3 & 2 \\
\hline 20,83 & $\mathrm{C}_{15} \mathrm{H}_{11} \mathrm{~N}$ & $3531-24-6$ & 2-Propenenitrile, 3,3-diphenyl- & 3 & 2 \\
\hline 20,81 & $\mathrm{C}_{17} \mathrm{H}_{3} 6 \mathrm{O}_{2} \mathrm{Si}$ & $18603-17-3$ & Tetradecanoic acid, trimethylsilyl ester & 5 & 2 \\
\hline 19,34 & $\mathrm{C}_{8} \mathrm{H}_{14} \mathrm{O}_{4}$ & $627-93-0$ & Hexanedioic acid, dimethyl ester & 5 & 3 \\
\hline \multirow[t]{24}{*}{13,44} & $\mathrm{C}_{6} \mathrm{H}_{7} \mathrm{NS}$ & $18438-38-5$ & Pyridine, 2-(methylthio)- & 4 & 2 \\
\hline & $\mathrm{C}_{1} 8 \mathrm{H}_{2} 8 \mathrm{~N}_{2} \mathrm{O}_{4}$ & & Acebutolol & 3 & 2 \\
\hline & $\mathrm{C}_{2} \mathrm{OH}_{23} \mathrm{~N}$ & & Amitriptyline & 6 & 2 \\
\hline & $\mathrm{C}_{2} \mathrm{OH}_{2} \mathrm{ClN}_{2} \mathrm{O}_{5}$ & & Amlodipine & 6 & 2 \\
\hline & $\mathrm{C}_{3} 8 \mathrm{H}_{52} \mathrm{~N}_{6} \mathrm{O}_{7}$ & & Atazanavir & 5 & 2 \\
\hline & $\mathrm{C}_{14} \mathrm{H}_{22} \mathrm{~N}_{2} \mathrm{O}_{3}$ & & Atenolol & 7 & 2 \\
\hline & $\mathrm{C}_{3} 8 \mathrm{H}_{72} \mathrm{~N}_{2} \mathrm{O}_{12}$ & & Azithromycin & 6 & 2 \\
\hline & $\mathrm{C}_{1} 6 \mathrm{H}_{19} \mathrm{NO}_{4}$ & & Benzoylecgonine & 5 & 2 \\
\hline & $\mathrm{C}_{1} 8 \mathrm{H}_{31} \mathrm{NO}_{4}$ & & Bisoprolol & 4 & 2 \\
\hline & $\mathrm{C}_{8} \mathrm{H}_{1} \mathrm{NN}_{4} \mathrm{O}_{2}$ & & Caffeine & 7 & 2 \\
\hline & $\mathrm{C}_{15} \mathrm{H}_{12} \mathrm{~N}_{2} \mathrm{O}$ & & Carbamazepine & 7 & 2 \\
\hline & $\mathrm{C}_{15} \mathrm{H}_{14} \mathrm{~N}_{2} \mathrm{O}_{3}$ & & Carbamazepine, 10, 11-dihydroxy & 7 & 2 \\
\hline & $\mathrm{C}_{15} \mathrm{H}_{14} \mathrm{~N}_{2} \mathrm{O}_{2}$ & & Carbamazepine, 10 -hydroxy & 7 & 2 \\
\hline & $\mathrm{C}_{2} \mathrm{OH}_{33} \mathrm{~N}_{3} \mathrm{O}_{4}$ & & Celiprolol & 4 & 2 \\
\hline & $\mathrm{C}_{21} \mathrm{H}_{25} \mathrm{ClN}_{2} \mathrm{O}_{3}$ & & Cetirizine & 7 & 2 \\
\hline & $\mathrm{C}_{17} \mathrm{H}_{1} 8 \mathrm{FN}_{3} \mathrm{O}_{3}$ & & Ciprofloxacin & 7 & 2 \\
\hline & $\mathrm{C}_{19} \mathrm{H}_{19} \mathrm{FN}_{2} \mathrm{O}$ & & Citalopram, Desmethyl & 4 & 2 \\
\hline & $\mathrm{C}_{2} \mathrm{OH}_{21} \mathrm{FN} 2 \mathrm{O}$ & & Citalopram/Escitalopram & 7 & 2 \\
\hline & $\mathrm{C}_{3} 8 \mathrm{H}_{69} \mathrm{NO}_{13}$ & & Clarithromycin & 6 & 2 \\
\hline & $\mathrm{C}_{18} \mathrm{H}_{19} \mathrm{ClN}_{4}$ & & Clozapine & 7 & 2 \\
\hline & $\mathrm{C}_{17} \mathrm{H}_{17} \mathrm{ClN}_{4}$ & & Clozapine, Desmethyl & 6 & 2 \\
\hline & $\mathrm{C}_{17} \mathrm{H}_{21} \mathrm{NO}_{4}$ & & Cocaine & 5 & 2 \\
\hline & $\mathrm{C}_{1} 8 \mathrm{H}_{21} \mathrm{NO}_{3}$ & & Codeine & 6 & 2 \\
\hline & $\mathrm{C}_{1} \mathrm{OH}_{12} \mathrm{~N}_{2} \mathrm{O}$ & & Cotinine & 7 & 2 \\
\hline
\end{tabular}


Continued

\begin{tabular}{|c|c|c|c|c|c|}
\hline $\begin{array}{l}\text { Quant } \\
\text { (ng/L) }\end{array}$ & $\begin{array}{l}\text { Molecular } \\
\text { Formula }\end{array}$ & CAS & Common name & $\begin{array}{l}\text { Occur/ } \\
\text { matrix }\end{array}$ & $\begin{array}{r}\text { ID } \\
\text { Level }\end{array}$ \\
\hline & $\mathrm{C}_{22} \mathrm{H}_{2} 6 \mathrm{~N}_{2} \mathrm{O}_{4} \mathrm{~S}$ & & Diltiazem & 6 & 2 \\
\hline & $\mathrm{C}_{2} 4 \mathrm{H}_{4} \mathrm{ON}_{8} \mathrm{O}_{4}$ & & Dipyridamol & 4 & 2 \\
\hline & $\mathrm{C}_{23} \mathrm{H}_{24} \mathrm{~N}_{2} \mathrm{O}_{4} \mathrm{~S}$ & & Eprosartan & 3 & 2 \\
\hline & $\mathrm{C}_{32} \mathrm{H}_{39} \mathrm{NO}_{4}$ & & Fexofenadine & 7 & 2 \\
\hline & $\mathrm{C}_{17} \mathrm{H}_{2} \mathrm{OF} 6 \mathrm{~N}_{2} \mathrm{O}_{3}$ & & Flecainide & 5 & 2 \\
\hline & $\mathrm{C}_{1} 8 \mathrm{H}_{21} \mathrm{NO}_{3}$ & & Hydrocodone & 6 & 2 \\
\hline & $\mathrm{C}_{25} \mathrm{H}_{28} 8 \mathrm{~N} 6 \mathrm{O}$ & & Irbesartan & 4 & 2 \\
\hline & $\mathrm{C}_{2} 6 \mathrm{H}_{2} 8 \mathrm{Cl}_{2} \mathrm{~N}_{4} \mathrm{O}_{4}$ & & Ketoconazole & 7 & 2 \\
\hline & $\mathrm{C}_{9} \mathrm{H}_{7} \mathrm{Cl}_{2} \mathrm{~N}_{5}$ & & Lamotrigine & 7 & 2 \\
\hline & $\mathrm{C}_{22} \mathrm{H}_{23} \mathrm{ClN} 6 \mathrm{O}$ & & Losartan & 7 & 2 \\
\hline & $\mathrm{C}_{2} \mathrm{OH}_{23} \mathrm{~N}$ & & Maprotiline & 6 & 2 \\
\hline & $\mathrm{C}_{11} \mathrm{H}_{15} \mathrm{NO}_{2}$ & & MDMA & 5 & 2 \\
\hline & $\mathrm{C}_{21} \mathrm{H}_{27} \mathrm{NO}$ & & Methadone & 4 & 2 \\
\hline & $\mathrm{C}_{14} \mathrm{H}_{22} \mathrm{ClN}_{3} \mathrm{O}_{2}$ & & Metoclopramide & 6 & 2 \\
\hline & $\mathrm{C}_{15} \mathrm{H}_{25} \mathrm{NO}_{3}$ & & Metoprolol & 7 & 2 \\
\hline & $\mathrm{C}_{17} \mathrm{H}_{19} \mathrm{~N}_{3}$ & & Mirtazapine & 7 & 2 \\
\hline & $\mathrm{C}_{10 \mathrm{H}_{14} \mathrm{~N}_{2}}$ & & Nicotine & 7 & 2 \\
\hline & $\mathrm{C}_{15} \mathrm{H}_{11} \mathrm{ClN}_{2} \mathrm{O}_{2}$ & & Oxazepam & 6 & 2 \\
\hline & $\mathrm{C}_{1} 6 \mathrm{H}_{21} \mathrm{NO}_{2}$ & & Propranolol & 7 & 2 \\
\hline & $\mathrm{C}_{21} \mathrm{H}_{25} \mathrm{~N}_{3} \mathrm{O}_{2} \mathrm{~S}$ & & Quetiapine & 7 & 2 \\
\hline & $\mathrm{C}_{2} \mathrm{OH}_{2} \mathrm{~N}_{2} \mathrm{O}_{2}$ & & Quinine & 7 & 2 \\
\hline & $\mathrm{C}_{16} \mathrm{H}_{15} \mathrm{~F}_{6} \mathrm{~N}_{5} \mathrm{O}$ & & Sitagliptin & 5 & 2 \\
\hline & $\mathrm{C}_{12} \mathrm{H}_{2} \mathrm{ON}_{2} \mathrm{O}_{3} \mathrm{~S}$ & & Sotalol & 6 & 2 \\
\hline & $\mathrm{C}_{11} \mathrm{H}_{11} \mathrm{~N}_{3} \mathrm{O}_{2} \mathrm{~S}$ & & Sulfapyridine & 7 & 2 \\
\hline & $\mathrm{C}_{15} \mathrm{H}_{23} \mathrm{~N}_{3} \mathrm{O}_{4} \mathrm{~S}$ & & Sulpiride & 4 & 2 \\
\hline & $\mathrm{C}_{14} \mathrm{H}_{21} \mathrm{~N}_{3} \mathrm{O}_{2} \mathrm{~S}$ & & Sumatriptan & 5 & 2 \\
\hline & $\mathrm{C}_{33} \mathrm{H}_{3} \mathrm{ON}_{4} \mathrm{O}_{2}$ & & Telmisartan & 6 & 2 \\
\hline & $\mathrm{C}_{1} 6 \mathrm{H}_{25} \mathrm{NO}_{2}$ & & Tramadol & 7 & 2 \\
\hline & $\mathrm{C}_{14} \mathrm{H}_{1} 8 \mathrm{~N}_{4} \mathrm{O}_{3}$ & & Trimethoprim & 8 & 2 \\
\hline & $\mathrm{C}_{2}{ }_{4} \mathrm{H}_{29} \mathrm{~N}_{5} \mathrm{O}_{3}$ & & Valsartan & 5 & 2 \\
\hline & $\mathrm{C}_{17} \mathrm{H}_{27} \mathrm{NO}_{2}$ & & Venlafaxine & 7 & 2 \\
\hline & $\mathrm{C}_{1} 6 \mathrm{H}_{25} \mathrm{NO}_{2}$ & & Venlafaxine,-O-demethyl- & 7 & 2 \\
\hline & $\mathrm{C}_{17} \mathrm{H}_{2} \mathrm{ON}_{4} \mathrm{~S}$ & $132539-06-1$ & OLANZAPINE & 1 & 3 \\
\hline & 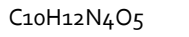 & $58-63-9$ & Inosine & 1 & 3 \\
\hline & $\mathrm{C}_{13} \mathrm{H}_{13} \mathrm{~N}_{3} \mathrm{O}_{3} \mathrm{~S}$ & $19077-98-6$ & N-Acetylsulfapyridin & 2 & 3 \\
\hline & $\mathrm{C}_{7} \mathrm{H}_{8} \mathrm{ClN}_{3} \mathrm{O}_{4} \mathrm{~S}_{2}$ & $58-93-5$ & HYDROCHLOROTHIAZIDE & 5 & 3 \\
\hline & $\mathrm{C}_{12} \mathrm{H}_{2} 6 \mathrm{O}_{4} \mathrm{~S}$ & $4706-78-9$ & dodecyl sulfuric acid & 3 & 3 \\
\hline & $\mathrm{C}_{12} \mathrm{H}_{11} \mathrm{~N}_{3} \mathrm{O}_{3} \mathrm{~S}$ & $104-23-4$ & 4'-aminoazobenzene-4-sulfonic acid & 2 & 3 \\
\hline & $\mathrm{C}_{13} \mathrm{H}_{10} \mathrm{~N}_{2} \mathrm{O}_{3} \mathrm{~S}$ & $27503-81-7$ & 2-phenyl-1H-benzimidazole-5-sulphonic acid & 7 & 3 \\
\hline & $\mathrm{C}_{17} \mathrm{H}_{19} \mathrm{~N}_{3} \mathrm{O}_{4} \mathrm{~S}$ & $88546-55-8$ & Omeprazole Sulfone & 1 & 3 \\
\hline & & $40143-76-8$ & PFHXPA & 2 & 3 \\
\hline & & $2043-47-2$ & $4: 2 \mathrm{FTOH}$ & 1 & 3 \\
\hline & & $620-92-8$ & $4,4-B P F$ & 4 & 2 \\
\hline & & $137862-53-4$ & Valsartan & 3 & 3 \\
\hline & & $4065-45-6$ & benzophenone-4 & 5 & 3 \\
\hline & & $27503-81-7$ & PBS (Phenylbenzimidazole sulfonic acid) & 7 & 3 \\
\hline & & $58-93-5$ & Hydrochlorothiazide & 5 & 3 \\
\hline & & $123-08-0$ & 4-hydroxybenzaldehyde & 7 & 3 \\
\hline
\end{tabular}


Table 12: Effluent Sweden

\begin{tabular}{|c|c|c|c|c|c|}
\hline $\begin{array}{l}\text { Quant } \\
\text { (ng/L) }\end{array}$ & $\begin{array}{l}\text { Molecular } \\
\text { Formula }\end{array}$ & CAS & Common name & $\begin{array}{l}\text { Occur/ } \\
\text { matrix }\end{array}$ & $\begin{array}{r}\text { ID } \\
\text { Level }\end{array}$ \\
\hline 3824,61 & $\mathrm{C}_{19} \mathrm{H}_{4} \mathrm{OOO}_{2} \mathrm{Si}$ & $55520-89-3$ & Hexadecanoic acid, trimethylsilyl ester & 7 & 2 \\
\hline 1102,76 & $\mathrm{C}_{10 \mathrm{H}} 6$ & $3760-14-3$ & 1,5-Cyclooctadiene, 1,5-dimethyl- & 7 & 3 \\
\hline 1046,77 & $\mathrm{C}_{21} \mathrm{H}_{44} \mathrm{O}_{2} \mathrm{Si}$ & $18748-91-9$ & Octadecanoic acid, trimethylsilyl ester & 4 & 2 \\
\hline 329,05 & $\mathrm{C}_{1} 8 \mathrm{H}_{2} 6 \mathrm{O}$ & $1222-05-5$ & Galaxolide & 6 & 2 \\
\hline 297,52 & $\mathrm{C}_{1} 8 \mathrm{H}_{3} 8 \mathrm{O}_{2} \mathrm{Si}$ & $74367-22-9$ & n-Pentadecanoic acid, trimethylsilyl ester & 4 & 2 \\
\hline 273,84 & $\mathrm{C}_{15} \mathrm{H}_{13} \mathrm{~N}$ & $2286-54-6$ & Benzenepropanenitrile, á-phenyl- & 5 & 2 \\
\hline 203,31 & $\mathrm{C}_{17} \mathrm{H}_{3} 6 \mathrm{O}_{2} \mathrm{Si}$ & $18603-17-3$ & Tetradecanoic acid, trimethylsilyl ester & 5 & 2 \\
\hline 201,91 & $\mathrm{C}_{7} \mathrm{H} 8 \mathrm{O}$ & $108-39-4$ & Phenol, 3-methyl- & 3 & 2 \\
\hline 198,79 & $\mathrm{C}_{9} \mathrm{H}_{18} 8 \mathrm{Cl}_{3} \mathrm{O}_{4} \mathrm{P}$ & $13674-84-5$ & 2-Propanol, 1-chloro-, phosphate (3:1) & 4 & 2 \\
\hline 189,35 & $\mathrm{C}_{14} \mathrm{H}_{11} \mathrm{~N}$ & $611-64-3$ & Acridine, 9-methyl- & 5 & 2 \\
\hline 159,37 & $\mathrm{C}_{8} \mathrm{H}_{10 O}$ & $122-99-6$ & Ethanol, 2-phenoxy- & 6 & 2 \\
\hline 120,70 & $\mathrm{C} 8 \mathrm{H}_{6} \mathrm{O}_{2}$ & $1074-12-0$ & Phenylglyoxal & 6 & 2 \\
\hline 104,57 & $\mathrm{C}_{13} \mathrm{H}_{10} \mathrm{O}$ & $119-61-9$ & Benzophenone & 7 & 2 \\
\hline 101,37 & $\mathrm{C}_{8} \mathrm{H}_{7} \mathrm{NS}_{2}$ & $615-22-5$ & Benzothiazole, 2-(methylthio)- & 5 & 2 \\
\hline 92,19 & $\mathrm{C}_{12} \mathrm{H}_{24} \mathrm{O}_{3}$ & $74367-34-3$ & Propanoic acid, 2-methyl-, 3-hydroxy-2,4,4-trimethylpentyl ester & 7 & 2 \\
\hline 88,30 & $\mathrm{C}_{14} \mathrm{H}_{14} \mathrm{O}$ & $19578-70-2$ & Benzene, 1-methyl-2-(phenylmethoxy)- & 5 & 2 \\
\hline 86,69 & $\mathrm{C}_{14} \mathrm{H}_{9} \mathrm{Cl}_{2} \mathrm{NO}$ & $15362-40-0$ & 2H-Indol-2-one, 1-(2,6-dichlorophenyl)-1,3-dihydro- & 3 & 3 \\
\hline 85,51 & $\mathrm{C}_{2} \mathrm{OH}_{32} \mathrm{O}_{2}$ & - & Benzoic acid, tridecyl ester & 7 & 3 \\
\hline 85,03 & $\mathrm{C}_{1} 8 \mathrm{H}_{2} 8 \mathrm{O}_{2}$ & $6316-30-9$ & Benzoic acid, undecyl ester & 7 & 3 \\
\hline 76,10 & $\mathrm{C} 8 \mathrm{H}_{8} \mathrm{O}_{2}$ & $582-24-1$ & Ethanone, 2-hydroxy-1-phenyl- & 7 & 2 \\
\hline 69,85 & $\mathrm{C}_{9} \mathrm{H}_{14} \mathrm{O} 6$ & $102-76-1$ & Triacetin & 4 & 2 \\
\hline 63,63 & $\mathrm{C}_{7} \mathrm{H}_{9} \mathrm{NO}_{2}$ & $20189-42-8$ & 1H-Pyrrole-2,5-dione, 3-ethyl-4-methyl- & 7 & 3 \\
\hline 61,76 & $\mathrm{C}_{8} \mathrm{H}_{10} \mathrm{~N}_{4} \mathrm{O}_{2}$ & $58-08-2$ & Caffeine & 7 & 3 \\
\hline 58,09 & $\mathrm{C}_{22} \mathrm{H}_{3} 6 \mathrm{O}_{2}$ & - & Benzoic acid, pentadecyl ester & 6 & 3 \\
\hline 33,42 & $\mathrm{C}_{10} \mathrm{H}_{14} \mathrm{O}$ & $99-49-0$ & Carvone & 5 & 2 \\
\hline 32,81 & $\mathrm{C}_{11} \mathrm{H}_{13} \mathrm{ClO}$ & - & 3-Buten-1-ol, 4-chloro-2-methyl-1-phenyl- & 3 & 2 \\
\hline 31,21 & $\mathrm{C}_{6} \mathrm{H}_{7} \mathrm{NS}$ & $18438-38-5$ & Pyridine, 2-(methylthio)- & 4 & 2 \\
\hline 21,19 & $\mathrm{C}_{8} \mathrm{H}_{14} \mathrm{O}_{4}$ & $627-93-0$ & Hexanedioic acid, dimethyl ester & 5 & 3 \\
\hline 16,27 & $\mathrm{C}_{7} \mathrm{H}_{5} \mathrm{NOS}$ & $934-34-9$ & $2(3 \mathrm{H})$-Benzothiazolone & 2 & 3 \\
\hline 14,35 & $\mathrm{C}_{1} \mathrm{OH}_{22} \mathrm{O}_{3}$ & $29911-28-2$ & 2-Propanol, 1-(2-butoxy-1-methylethoxy)- & 5 & 3 \\
\hline 10,15 & $\mathrm{C}_{5} \mathrm{H}_{9} \mathrm{NO}_{2}$ & $4394-85-8$ & N-Formylmorpholine & 3 & 2 \\
\hline \multirow[t]{32}{*}{9,43} & $\mathrm{C}_{11} \mathrm{H}_{10}$ & $90-12-0$ & Naphthalene, 1-methyl- & 2 & 2 \\
\hline & $\mathrm{C}_{1} 8 \mathrm{H}_{2} 8 \mathrm{~N}_{2} \mathrm{O}_{4}$ & & Acebutolol & 3 & 2 \\
\hline & $\mathrm{C}_{2} \mathrm{OH} 23 \mathrm{~N}$ & & Amitriptyline & 6 & 2 \\
\hline & $\mathrm{C}_{2} \mathrm{OH}_{25} \mathrm{ClN}_{2} \mathrm{O}_{5}$ & & Amlodipine & 6 & 2 \\
\hline & $\mathrm{C}_{3} 8 \mathrm{H}_{52} \mathrm{~N}_{6} \mathrm{O}_{7}$ & & Atazanavir & 5 & 2 \\
\hline & $\mathrm{C}_{14} \mathrm{H}_{22} \mathrm{~N}_{2} \mathrm{O}_{3}$ & & Atenolol & 7 & 2 \\
\hline & $\mathrm{C}_{3} 8 \mathrm{H}_{72} \mathrm{~N}_{2} \mathrm{O}_{12}$ & & Azithromycin & 6 & 2 \\
\hline & $\mathrm{C}_{16} \mathrm{H}_{19} \mathrm{NO}_{4}$ & & Benzoylecgonine & 5 & 2 \\
\hline & $\mathrm{C}_{1} 8 \mathrm{H}_{31} \mathrm{NO}_{4}$ & & Bisoprolol & 4 & 2 \\
\hline & $\mathrm{C}_{8} \mathrm{H}_{10} \mathrm{~N}_{4} \mathrm{O}_{2}$ & & Caffeine & 7 & 2 \\
\hline & $\mathrm{C}_{15} \mathrm{H}_{12} \mathrm{~N}_{2} \mathrm{O}$ & & Carbamazepine & 7 & 2 \\
\hline & $\mathrm{C}_{15} \mathrm{H}_{14} \mathrm{~N}_{2} \mathrm{O}_{3}$ & & Carbamazepine, 10, 11-dihydroxy & 7 & 2 \\
\hline & $\mathrm{C}_{15} \mathrm{H}_{14} \mathrm{~N}_{2} \mathrm{O}_{2}$ & & Carbamazepine, 10-hydroxy & 7 & 2 \\
\hline & $\mathrm{C}_{2} \mathrm{OH}_{3} \mathrm{~N}_{3} \mathrm{O}_{4}$ & & Celiprolol & 4 & 2 \\
\hline & $\mathrm{C}_{21} \mathrm{H}_{25} \mathrm{ClN}_{2} \mathrm{O}_{3}$ & & Cetirizine & 7 & 2 \\
\hline & $\mathrm{C}_{17} \mathrm{H}_{1} 8 \mathrm{FN}_{3} \mathrm{O}_{3}$ & & Ciprofloxacin & 7 & 2 \\
\hline & $\mathrm{C}_{19} \mathrm{H}_{19} \mathrm{FN}_{2} \mathrm{O}$ & & Citalopram, Desmethyl & 4 & 2 \\
\hline & $\mathrm{C}_{2} \mathrm{OH}_{21} \mathrm{FN} 2 \mathrm{O}$ & & Citalopram/Escitalopram & 7 & 2 \\
\hline & $\mathrm{C}_{3} 8 \mathrm{H}_{69} \mathrm{NO}_{13}$ & & Clarithromycin & 6 & 2 \\
\hline & $\mathrm{C}_{18} \mathrm{H}_{19} \mathrm{ClN}_{4}$ & & Clozapine & 7 & 2 \\
\hline & $\mathrm{C}_{17} \mathrm{H}_{17} \mathrm{CIN}_{4}$ & & Clozapine, Desmethyl & 6 & 2 \\
\hline & $\mathrm{C}_{17} \mathrm{H}_{21} \mathrm{NO}_{4}$ & & Cocaine & 5 & 2 \\
\hline & $\mathrm{C}_{1} 8 \mathrm{H}_{21} \mathrm{NO}_{3}$ & & Codeine & 6 & 2 \\
\hline & $\mathrm{C}_{1} \mathrm{OH}_{12} \mathrm{~N}_{2} \mathrm{O}$ & & Cotinine & 7 & 2 \\
\hline & $\mathrm{C}_{22} \mathrm{H}_{2} 6 \mathrm{~N}_{2} \mathrm{O}_{4} \mathrm{~S}$ & & Diltiazem & 6 & 2 \\
\hline & $\mathrm{C}_{23} \mathrm{H}_{24} \mathrm{~N}_{2} \mathrm{O}_{4} \mathrm{~S}$ & & Eprosartan & 3 & 2 \\
\hline & $\mathrm{C}_{32} \mathrm{H}_{39} \mathrm{NO}_{4}$ & & Fexofenadine & 7 & 2 \\
\hline & $\mathrm{C}_{17} \mathrm{H}_{2} \mathrm{OF} 6 \mathrm{~N}_{2} \mathrm{O}_{3}$ & & Flecainide & 5 & 2 \\
\hline & $\mathrm{C}_{1} 8 \mathrm{H}_{21} \mathrm{NO}_{3}$ & & Hydrocodone & 6 & 2 \\
\hline & $\mathrm{C}_{2} 6 \mathrm{H}_{2} 8 \mathrm{Cl}_{2} \mathrm{~N}_{4} \mathrm{O}_{4}$ & & Ketoconazole & 7 & 2 \\
\hline & $\mathrm{C}_{9} \mathrm{H}_{7} \mathrm{Cl}_{2} \mathrm{~N}_{5}$ & & Lamotrigine & 7 & 2 \\
\hline & $\mathrm{C}_{22} \mathrm{H}_{23} \mathrm{ClN} 6 \mathrm{O}$ & & Losartan & 7 & 2 \\
\hline
\end{tabular}


Continued

\begin{tabular}{|c|c|c|c|c|c|}
\hline $\begin{array}{l}\text { Quant } \\
\text { (ng/L) }\end{array}$ & $\begin{array}{l}\text { Molecular } \\
\text { Formula }\end{array}$ & CAS & Common name & $\begin{array}{l}\text { Occur/ } \\
\text { matrix }\end{array}$ & $\begin{array}{r}\text { ID } \\
\text { Level }\end{array}$ \\
\hline & $\mathrm{C}_{2} \mathrm{OH}_{23} \mathrm{~N}$ & & Maprotiline & 6 & 2 \\
\hline & $\mathrm{C}_{11} \mathrm{H}_{15} \mathrm{NO}_{2}$ & & MDMA & 5 & 2 \\
\hline & $\mathrm{C}_{21} \mathrm{H}_{27} \mathrm{NO}$ & & Methadone & 4 & 2 \\
\hline & $\mathrm{C}_{14} \mathrm{H}_{22} \mathrm{ClN}_{3} \mathrm{O}_{2}$ & & Metoclopramide & 6 & 2 \\
\hline & $\mathrm{C}_{15} \mathrm{H}_{25} \mathrm{NO}_{3}$ & & Metoprolol & 7 & 2 \\
\hline & $\mathrm{C}_{17} \mathrm{H}_{19} \mathrm{~N}_{3}$ & & Mirtazapine & 7 & 2 \\
\hline & $\mathrm{C}_{10 \mathrm{H}_{1}} \mathrm{~N}_{2}$ & & Nicotine & 7 & 2 \\
\hline & $\mathrm{C}_{15} \mathrm{H}_{11} \mathrm{ClN}_{2} \mathrm{O}_{2}$ & & Oxazepam & 6 & 2 \\
\hline & $\mathrm{C}_{1} 6 \mathrm{H}_{21} \mathrm{NO}_{2}$ & & Propranolol & 7 & 2 \\
\hline & $\mathrm{C}_{21} \mathrm{H}_{25} \mathrm{~N}_{3} \mathrm{O}_{2} \mathrm{~S}$ & & Quetiapine & 7 & 2 \\
\hline & $\mathrm{C}_{2} \mathrm{OH}_{2}{ }_{4} \mathrm{~N}_{2} \mathrm{O}_{2}$ & & Quinine & 7 & 2 \\
\hline & $\mathrm{C}_{1} 6 \mathrm{H}_{15} \mathrm{~F}_{6} \mathrm{~N}_{5} \mathrm{O}$ & & Sitagliptin & 5 & 2 \\
\hline & $\mathrm{C}_{12} \mathrm{H}_{2} \mathrm{ON}_{2} \mathrm{O}_{3} \mathrm{~S}$ & & Sotalol & 6 & 2 \\
\hline & $\mathrm{C}_{11} \mathrm{H}_{11} \mathrm{~N}_{3} \mathrm{O}_{2} \mathrm{~S}$ & & Sulfapyridine & 7 & 2 \\
\hline & $\mathrm{C}_{15} \mathrm{H}_{23} \mathrm{~N}_{3} \mathrm{O}_{4} \mathrm{~S}$ & & Sulpiride & 4 & 2 \\
\hline & $\mathrm{C}_{14} \mathrm{H}_{21} \mathrm{~N}_{3} \mathrm{O}_{2} \mathrm{~S}$ & & Sumatriptan & 7 & 2 \\
\hline & $\mathrm{C}_{33} \mathrm{H}_{3} \mathrm{ON}_{4} \mathrm{O}_{2}$ & & Telmisartan & 5 & 2 \\
\hline & $\mathrm{C}_{1} 6 \mathrm{H}_{25} \mathrm{NO}_{2}$ & & Tramadol & 6 & 2 \\
\hline & $\mathrm{C}_{14} \mathrm{H}_{1} 8 \mathrm{~N}_{4} \mathrm{O}_{3}$ & & Trimethoprim & 7 & 2 \\
\hline & $\mathrm{C}_{24} \mathrm{H}_{29} \mathrm{~N}_{5} \mathrm{O}_{3}$ & & Valsartan & 8 & 2 \\
\hline & $\mathrm{C}_{17} \mathrm{H}_{27} \mathrm{NO}_{2}$ & & Venlafaxine & 9 & 2 \\
\hline & $\mathrm{C}_{1} 6 \mathrm{H}_{25} \mathrm{NO}_{2}$ & & Venlafaxine,-O-demethyl- & 7 & 2 \\
\hline & $\mathrm{C}_{2} 4 \mathrm{H}_{4} 8 \mathrm{O}_{2}$ & $13945-76-1$ & dodecyl dodecanoate & 1 & 2 \\
\hline & $\mathrm{C}_{2} \mathrm{OH}_{4} \mathrm{OO}_{2}$ & $506-30-9$ & icosanoic acid & 2 & 2 \\
\hline & $\mathrm{C}_{9} \mathrm{H}_{19} \mathrm{NO}_{4}$ & $16485-10-2$ & panthenol , DL-form & 2 & 2 \\
\hline & $\mathrm{C}_{18} \mathrm{H}_{14} \mathrm{~F}_{4} \mathrm{~N}_{2} \mathrm{O}_{4} \mathrm{~S}$ & & Bicalutamid & 3 & 2 \\
\hline & $\mathrm{C}_{9} \mathrm{H}_{11} \mathrm{NO}_{2}$ & $94-09-7$ & BENZOCAINE & 2 & 2 \\
\hline & $\mathrm{C}_{11} \mathrm{H}_{10} \mathrm{~N}_{4} \mathrm{O}_{4}$ & $6804-07-5$ & carbadox & 1 & 2 \\
\hline & $\mathrm{C}_{6} \mathrm{H}_{7} \mathrm{~N}_{5} \mathrm{~S}$ & $1198-47-6$ & 6-Methylthioguanin & 1 & 2 \\
\hline & $\mathrm{C}_{14} \mathrm{H}_{9} \mathrm{ClF}_{3} \mathrm{NO}_{3}$ & $205754-32-1$ & rac 8-Hydroxy Efavirenz & 1 & 2 \\
\hline & $\mathrm{C}_{13} \mathrm{H}_{13} \mathrm{~N}_{3} \mathrm{O}_{3} \mathrm{~S}$ & $19077-98-6$ & N-Acetylsulfapyridin & 2 & 2 \\
\hline & $\mathrm{C}_{14} \mathrm{H}_{12} \mathrm{O} 6 \mathrm{~S}$ & $4065-45-6$ & sulisobenzone & 5 & 2 \\
\hline & $\mathrm{C}_{5} \mathrm{H}_{8} \mathrm{O}_{3}$ & $105-45-3$ & methyl acetoacetate & 2 & 2 \\
\hline & $\mathrm{C}_{11} \mathrm{H}_{11} \mathrm{~N}_{3} \mathrm{O}_{2} \mathrm{~S}$ & $144-83-2$ & sulfapyridine & 1 & 2 \\
\hline & $\mathrm{C}_{22} \mathrm{H}_{43} \mathrm{NO}$ & & docos-13-enamide, Amides, C22 (unsaturated) & o & 2 \\
\hline & $\mathrm{C}_{13} \mathrm{H}_{10} \mathrm{~N}_{2} \mathrm{O}_{3} \mathrm{~S}$ & $27503-81-7$ & 2-phenyl-1H-benzimidazole-5-sulphonic acid & 7 & 2 \\
\hline & $\mathrm{C}_{8} \mathrm{H}_{5} \mathrm{NO}_{2}$ & $85-41-6$ & $1 \mathrm{H}$-Isoindole-1,3(2H)-dione & 2 & 2 \\
\hline & $\mathrm{C}_{7} \mathrm{H}_{9} \mathrm{NO}_{2}$ & $30652-11-0$ & Deferiprone & 2 & 2 \\
\hline & $\mathrm{C}_{2} \mathrm{HF}_{3} \mathrm{O}_{2}$ & $76-05-1$ & trifluoroacetic acid & 1 & 2 \\
\hline & $\mathrm{C}_{6} \mathrm{H}_{6} \mathrm{~N}_{4} \mathrm{O}_{2}$ & $552-62-5$ & 7-Methylxanthin & 1 & 2 \\
\hline & $\mathrm{C}_{12} \mathrm{H}_{11} \mathrm{~N}_{3} \mathrm{O}_{3} \mathrm{~S}$ & $104-23-4$ & 4'-aminoazobenzene-4-sulfonic acid & 2 & 2 \\
\hline & $\mathrm{C}_{1} 8 \mathrm{H}_{3} 6 \mathrm{O}_{2}$ & $57-11-4$ & Octadecanoic acid & 2 & 2 \\
\hline & $\mathrm{C}_{12} \mathrm{H}_{2} 6 \mathrm{O}_{4} \mathrm{~S}$ & $4706-78-9$ & dodecyl sulfuric acid & 3 & 2 \\
\hline & & $1799-84-4$ & 4:2 FTMAC & 5 & 2 \\
\hline & & $40143-76-8$ & PFHXPA & 2 & 2 \\
\hline & & $68259-15-4$ & $\mathrm{MeFH} \times \mathrm{SA}$ & 2 & 2 \\
\hline & & $620-92-8$ & $4,4-B P F$ & 4 & 1 \\
\hline & & $13595-25-0$ & bisphenol M & 5 & 1 \\
\hline & & $79-94-7$ & tetrabromobisphenol A & 1 & 2 \\
\hline & & $123-08-0$ & 4-hydroxybenzaldehyde & 7 & 2 \\
\hline & & $1074-86-8$ & 4-formyl indole & 6 & 2 \\
\hline & & $4065-45-6$ & benzophenone-4 (sulisobenzone) & 5 & 2 \\
\hline & & $27503-81-7$ & PBS (Phenylbenzimidazole sulfonic acid) & 7 & 2 \\
\hline & & $58-93-5$ & Hydrochlorothiazide & 5 & 2 \\
\hline & & $90357-06-5$ & bicalutamide & 3 & 2 \\
\hline
\end{tabular}


Table 13: Effluent Denmark

\begin{tabular}{|c|c|c|c|c|c|}
\hline $\begin{array}{l}\text { Quant } \\
\text { (ng/L) }\end{array}$ & $\begin{array}{l}\text { Molecular } \\
\text { Formula }\end{array}$ & CAS & Common name & $\begin{array}{l}\text { Occur/ } \\
\text { matrix }\end{array}$ & $\begin{array}{r}\text { ID } \\
\text { Level }\end{array}$ \\
\hline 1265,13 & $\mathrm{C}_{15} \mathrm{H}_{13} \mathrm{~N}$ & $2286-54-6$ & Benzenepropanenitrile, á-phenyl- & 5 & 2 \\
\hline 1250,26 & $\mathrm{C}_{1} 9 \mathrm{H}_{4} \mathrm{OO}_{2} \mathrm{Si}$ & $55520-89-3$ & Hexadecanoic acid, trimethylsilyl ester & 7 & 2 \\
\hline 319,42 & $\mathrm{C}_{14} \mathrm{H}_{11} \mathrm{~N}$ & $611-64-3$ & Acridine, 9-methyl- & 5 & 2 \\
\hline 317,09 & $\mathrm{C}_{10 \mathrm{H}_{1} 6}$ & $3760-14-3$ & 1,5-Cyclooctadiene, 1,5-dimethyl- & 7 & 3 \\
\hline 284,27 & $\mathrm{C} 8 \mathrm{H}_{6} \mathrm{O}_{2}$ & $1074-12-0$ & Phenylglyoxal & 6 & 2 \\
\hline 271,02 & $\mathrm{C}_{9} \mathrm{H}_{1} 8 \mathrm{Cl}_{3} \mathrm{O}_{4} \mathrm{P}$ & $13674-84-5$ & 2-Propanol, 1-chloro-, phosphate (3:1) & 4 & 2 \\
\hline 203,85 & $\mathrm{C}_{12} \mathrm{H}_{24} \mathrm{O}_{3}$ & $74367-34-3$ & Propanoic acid, 2-methyl-, 3-hydroxy-2,4,4-trimethylpentyl ester & 7 & 2 \\
\hline 200,60 & $\mathrm{C}_{1} 8 \mathrm{H}_{2} 6 \mathrm{O}$ & $1222-05-5$ & Galaxolide & 6 & 2 \\
\hline 177,27 & $\mathrm{C}_{1} \mathrm{OH}_{15} \mathrm{NO}_{2} \mathrm{~S}$ & $3622-84-2$ & Benzenesulfonamide, N-butyl- & 3 & 2 \\
\hline 146,93 & $\mathrm{C}_{8} \mathrm{H}_{7} \mathrm{NS}_{2}$ & $615-22-5$ & Benzothiazole, 2-(methylthio)- & 5 & 2 \\
\hline 101,28 & $\mathrm{C}_{13} \mathrm{H}_{10 O}$ & $119-61-9$ & Benzophenone & 7 & 2 \\
\hline 94,97 & $\mathrm{C} 8 \mathrm{H}_{8} \mathrm{O}_{2}$ & $582-24-1$ & Ethanone, 2-hydroxy-1-phenyl- & 7 & 2 \\
\hline 93,57 & $\mathrm{C}_{6} \mathrm{H}_{7} \mathrm{NS}$ & $18438-38-5$ & Pyridine, 2-(methylthio)- & 4 & 2 \\
\hline 87,05 & $\mathrm{C}_{7} \mathrm{H}_{9} \mathrm{NO}_{2}$ & $20189-42-8$ & 1H-Pyrrole-2,5-dione, 3-ethyl-4-methyl- & 7 & 3 \\
\hline 80,94 & $\mathrm{C}_{8} \mathrm{H}_{1} \mathrm{OO}_{2}$ & $122-99-6$ & Ethanol, 2-phenoxy- & 6 & 2 \\
\hline 66,76 & $\mathrm{C}_{12} \mathrm{H}_{18} \mathrm{O}$ & $2934-05-6$ & Phenol, 2,4-bis(1-methylethyl)- & 1 & 2 \\
\hline 66,06 & $\mathrm{C}_{15} \mathrm{H}_{11} \mathrm{~N}$ & $3531-24-6$ & 2-Propenenitrile, 3,3-diphenyl- & 3 & 2 \\
\hline 58,97 & $\mathrm{C}_{1} \mathrm{H}_{9} \mathrm{Cl}_{2} \mathrm{NO}$ & $15362-40-0$ & $2 \mathrm{H}$-Indol-2-one, 1-(2,6-dichlorophenyl)-1,3-dihydro- & 3 & 3 \\
\hline 56,11 & $\mathrm{C}_{2} \mathrm{OH}_{32} \mathrm{O}_{2}$ & - & Benzoic acid, tridecyl ester & 7 & 3 \\
\hline 54,28 & $\mathrm{C}_{1} 8 \mathrm{H}_{2} 8 \mathrm{O}_{2}$ & $6316-30-9$ & Benzoic acid, undecyl ester & 7 & 3 \\
\hline 47,08 & $\mathrm{C}_{8} \mathrm{H}_{10} \mathrm{~N}_{4} \mathrm{O}_{2}$ & $58-08-2$ & Caffeine & 7 & 3 \\
\hline \multirow[t]{41}{*}{39,73} & $\mathrm{C}_{22} \mathrm{H}_{3} 6 \mathrm{O}_{2}$ & - & Benzoic acid, pentadecyl ester & 6 & 3 \\
\hline & $\mathrm{C}_{2} \mathrm{OH}_{23} \mathrm{~N}$ & & Amitriptyline & 6 & 2 \\
\hline & $\mathrm{C}_{2} \mathrm{OH}_{25} \mathrm{ClN}_{2} \mathrm{O}_{5}$ & & Amlodipine & 6 & 2 \\
\hline & $\mathrm{C}_{3} 8 \mathrm{H}_{52} \mathrm{~N}_{6} \mathrm{O}_{7}$ & & Atazanavir & 5 & 2 \\
\hline & $\mathrm{C}_{14} \mathrm{H}_{22} \mathrm{~N}_{2} \mathrm{O}_{3}$ & & Atenolol & 7 & 2 \\
\hline & $\mathrm{C}_{3} 8 \mathrm{H}_{72} \mathrm{~N}_{2} \mathrm{O}_{12}$ & & Azithromycin & 6 & 2 \\
\hline & $\mathrm{C}_{1} 6 \mathrm{H}_{19} \mathrm{NO}_{4}$ & & Benzoylecgonine & 5 & 2 \\
\hline & $\mathrm{C}_{1} 8 \mathrm{H}_{31} \mathrm{NO}_{4}$ & & Bisoprolol & 4 & 2 \\
\hline & $\mathrm{C}_{8} \mathrm{H}_{10} \mathrm{~N}_{4} \mathrm{O}_{2}$ & & Caffeine & 7 & 2 \\
\hline & $\mathrm{C}_{15} \mathrm{H}_{12} \mathrm{~N}_{2} \mathrm{O}$ & & Carbamazepine & 7 & 2 \\
\hline & $\mathrm{C}_{15} \mathrm{H}_{14} \mathrm{~N}_{2} \mathrm{O}_{3}$ & & Carbamazepine, 10, 11-dihydroxy & 7 & 2 \\
\hline & $\mathrm{C}_{15} \mathrm{H}_{14} \mathrm{~N}_{2} \mathrm{O}_{2}$ & & Carbamazepine, 10-hydroxy & 7 & 2 \\
\hline & $\mathrm{C}_{2} \mathrm{OH}_{33} \mathrm{~N}_{3} \mathrm{O}_{4}$ & & Celiprolol & 4 & 2 \\
\hline & $\mathrm{C}_{21} \mathrm{H}_{25} \mathrm{ClN}_{2} \mathrm{O}_{3}$ & & Cetirizine & 7 & 2 \\
\hline & $\mathrm{C}_{17} \mathrm{H}_{1} 8 \mathrm{FN}_{3} \mathrm{O}_{3}$ & & Ciprofloxacin & 7 & 2 \\
\hline & $\mathrm{C}_{19} \mathrm{H}_{19} \mathrm{FN} 2 \mathrm{O}$ & & Citalopram, Desmethyl & 4 & 2 \\
\hline & $\mathrm{C}_{2} \mathrm{OH}_{21} \mathrm{FN}_{2} \mathrm{O}$ & & Citalopram/Escitalopram & 7 & 2 \\
\hline & $\mathrm{C}_{3} 8 \mathrm{H}_{69} \mathrm{NO}_{13}$ & & Clarithromycin & 6 & 2 \\
\hline & $\mathrm{C}_{18} \mathrm{H}_{19} \mathrm{ClN}_{4}$ & & Clozapine & 7 & 2 \\
\hline & $\mathrm{C}_{17} \mathrm{H}_{17} \mathrm{CIN}_{4}$ & & Clozapine, Desmethyl & 6 & 2 \\
\hline & $\mathrm{C}_{17} \mathrm{H}_{21} \mathrm{NO}_{4}$ & & Cocaine & 5 & 2 \\
\hline & $\mathrm{C}_{1} 8 \mathrm{H}_{21} \mathrm{NO}_{3}$ & & Codeine & 6 & 2 \\
\hline & $\mathrm{C}_{1} \mathrm{OH}_{12} \mathrm{~N}_{2} \mathrm{O}$ & & Cotinine & 7 & 2 \\
\hline & $\mathrm{C}_{22} \mathrm{H}_{2} 6 \mathrm{~N}_{2} \mathrm{O}_{4} \mathrm{~S}$ & & Diltiazem & 6 & 2 \\
\hline & $\mathrm{C}_{32} \mathrm{H}_{39} \mathrm{NO}_{4}$ & & Fexofenadine & 7 & 2 \\
\hline & $\mathrm{C}_{17} \mathrm{H}_{2} \mathrm{O} \mathrm{F}_{6} \mathrm{~N}_{2} \mathrm{O}_{3}$ & & Flecainide & 5 & 2 \\
\hline & $\mathrm{C}_{1} 8 \mathrm{H}_{21} \mathrm{NO}_{3}$ & & Hydrocodone & 6 & 2 \\
\hline & $\mathrm{C}_{25} \mathrm{H}_{2} 8 \mathrm{~N} 6 \mathrm{O}$ & & Irbesartan & 4 & 2 \\
\hline & $\mathrm{C}_{2} 6 \mathrm{H}_{2} 8 \mathrm{Cl}_{2} \mathrm{~N}_{4} \mathrm{O}_{4}$ & & Ketoconazole & 7 & 2 \\
\hline & $\mathrm{C}_{9} \mathrm{H}_{7} \mathrm{Cl}_{2} \mathrm{~N}_{5}$ & & Lamotrigine & 7 & 2 \\
\hline & $\mathrm{C}_{22} \mathrm{H}_{23} \mathrm{CIN} 6 \mathrm{O}$ & & Losartan & 7 & 2 \\
\hline & $\mathrm{C}_{2} \mathrm{OH}_{23} \mathrm{~N}$ & & Maprotiline & 6 & 2 \\
\hline & $\mathrm{C}_{11} \mathrm{H}_{15} \mathrm{NO}_{2}$ & & MDMA & 5 & 2 \\
\hline & $\mathrm{C}_{21} \mathrm{H}_{27} \mathrm{NO}$ & & Methadone & 4 & 2 \\
\hline & $\mathrm{C}_{14} \mathrm{H}_{22} \mathrm{ClN}_{3} \mathrm{O}_{2}$ & & Metoclopramide & 6 & 2 \\
\hline & $\mathrm{C}_{15} \mathrm{H}_{25} \mathrm{NO}_{3}$ & & Metoprolol & 7 & 2 \\
\hline & $\mathrm{C}_{17} \mathrm{H}_{19} \mathrm{~N}_{3}$ & & Mirtazapine & 7 & 2 \\
\hline & $\mathrm{C}_{10 \mathrm{H}_{1}} \mathrm{~N}_{2}$ & & Nicotine & 7 & 2 \\
\hline & $\mathrm{C}_{15} \mathrm{H}_{11} \mathrm{ClN}_{2} \mathrm{O}_{2}$ & & Oxazepam & 6 & 2 \\
\hline & $\mathrm{C}_{1} 6 \mathrm{H}_{21} \mathrm{NO}_{2}$ & & Propranolol & 7 & 2 \\
\hline & $\mathrm{C}_{21} \mathrm{H}_{25} \mathrm{~N}_{3} \mathrm{O}_{2} \mathrm{~S}$ & & Quetiapine & 7 & 2 \\
\hline
\end{tabular}


Continued

\begin{tabular}{|c|c|c|c|c|c|}
\hline $\begin{array}{l}\text { Quant } \\
\text { (ng/L) }\end{array}$ & $\begin{array}{l}\text { Molecular } \\
\text { Formula }\end{array}$ & CAS & Common name & $\begin{array}{c}\text { Occur/ } \\
\text { matrix }\end{array}$ & $\begin{array}{r}\text { ID } \\
\text { Level }\end{array}$ \\
\hline & $\mathrm{C}_{2} \mathrm{OH}_{24} \mathrm{~N}_{2} \mathrm{O}_{2}$ & & Quinine & 7 & 2 \\
\hline & $\mathrm{C}_{1} 6 \mathrm{H}_{15} \mathrm{~F}_{6} \mathrm{~N}_{5} \mathrm{O}$ & & Sitagliptin & 5 & 2 \\
\hline & $\mathrm{C}_{12} \mathrm{H}_{2} \mathrm{ON}_{2} \mathrm{O}_{3} \mathrm{~S}$ & & Sotalol & 6 & 2 \\
\hline & $\mathrm{C}_{11} \mathrm{H}_{11} \mathrm{~N}_{3} \mathrm{O}_{2} \mathrm{~S}$ & & Sulfapyridine & 7 & 2 \\
\hline & $\mathrm{C}_{15} \mathrm{H}_{23} \mathrm{~N}_{3} \mathrm{O}_{4} \mathrm{~S}$ & & Sulpiride & 4 & 2 \\
\hline & $\mathrm{C}_{14} \mathrm{H}_{21} \mathrm{~N}_{3} \mathrm{O}_{2} \mathrm{~S}$ & & Sumatriptan & 7 & 2 \\
\hline & $\mathrm{C}_{33} \mathrm{H}_{3} \mathrm{ON}_{4} \mathrm{O}_{2}$ & & Telmisartan & 5 & 2 \\
\hline & $\mathrm{C}_{1} 6 \mathrm{H}_{25} \mathrm{NO}_{2}$ & & Tramadol & 7 & 2 \\
\hline & $\mathrm{C}_{14} \mathrm{H}_{18} \mathrm{~N}_{4} \mathrm{O}_{3}$ & & Trimethoprim & 7 & 2 \\
\hline & $\mathrm{C}_{24} \mathrm{H}_{29} \mathrm{~N}_{5} \mathrm{O}_{3}$ & & Valsartan & 5 & 2 \\
\hline & $\mathrm{C}_{17} \mathrm{H}_{27} \mathrm{NO}_{2}$ & & Venlafaxine & 7 & 2 \\
\hline & $\mathrm{C}_{1} 6 \mathrm{H}_{25} \mathrm{NO}_{2}$ & & Venlafaxine,-O-demethyl- & 7 & 2 \\
\hline & $\mathrm{C}_{12} \mathrm{H}_{2} 6 \mathrm{O}_{4} \mathrm{~S}$ & 4706-78-9 & dodecyl sulfuric acid & 3 & 2 \\
\hline & $\mathrm{C}_{14} \mathrm{H}_{3} 0 \mathrm{O}_{4} \mathrm{~S}$ & $4754-44-3$ & tetradecyl sulfate & 1 & 2 \\
\hline & $\mathrm{C}_{1} 8 \mathrm{H}_{10}$ & $27208-37-3$ & Cyclopenta(c,d)pyren & 1 & 2 \\
\hline & $\mathrm{C}_{13} \mathrm{H}_{12} \mathrm{~F}_{2} \mathrm{~N} 6 \mathrm{O}$ & $86386-73-4$ & fluconazole & 1 & 2 \\
\hline & $\mathrm{C}_{9} \mathrm{H}_{1} 8 \mathrm{O}_{2}$ & $112-05-0$ & nonanoic acid & 1 & 2 \\
\hline & $\mathrm{C}_{9} \mathrm{H}_{1} 8 \mathrm{O}_{2}$ & $111-11-5$ & methyl octanoate & 1 & 2 \\
\hline & $\mathrm{C}_{9} \mathrm{H}_{1} 8 \mathrm{O}_{2}$ & $3302-10-1$ & 3,5,5-trimethylhexanoic acid & 1 & 2 \\
\hline & $\mathrm{C}_{9} \mathrm{H}_{18} 8 \mathrm{O}_{2}$ & $816-19-3$ & Methyl 2-ethylhexanoate & 1 & 2 \\
\hline & $\mathrm{C}_{9} \mathrm{H}_{1} 8 \mathrm{O}_{2}$ & $59354-78-8$ & neononanoic acid & 1 & 2 \\
\hline & $\mathrm{C}_{1} 6 \mathrm{H}_{3} \mathrm{OO}_{2}$ & $68227-33-8$ & $2,5,8,11$-tetramethyldodec-6-yne-5,8-diol & 1 & 2 \\
\hline & $\mathrm{C}_{1} 6 \mathrm{H}_{3} \mathrm{OO}_{2}$ & $142-90-5$ & dodecyl methacrylate & 2 & 2 \\
\hline & $\mathrm{C}_{2} \mathrm{OH}_{3} \mathrm{OO}_{2}$ & $14507-49-4$ & 13-ethyl-3-methoxygona-2,5(10)-dien-17 $\beta$-ol & 1 & 2 \\
\hline & $\mathrm{C}_{2} \mathrm{OH}_{3} \mathrm{OO}_{2}$ & $153-00-4$ & metenolone & 1 & 2 \\
\hline & $\mathrm{C}_{2} \mathrm{OH}_{3} \mathrm{OO}_{2}$ & $58-18-4$ & 17-alpha-Methyltestosterone & 1 & 2 \\
\hline & & $1799-84-4$ & 4:2 FTMAC & 5 & 2 \\
\hline & & $13595-25-0$ & bisphenol M & 5 & 1 \\
\hline & & $41340-25-4$ & etodolac (NSAID drug) & 1 & 2 \\
\hline & & $123-08-0$ & 4-hydroxybenzaldehyde & 7 & 2 \\
\hline & & $1074-86-8$ & 4-formyl indole & 6 & 2 \\
\hline & & $29878-31-7$ & 4-methylbenzotriazole (tolyltriazole) & 1 & 2 \\
\hline & & $4065-45-6$ & benzophenone-4 (sulisobenzone) & 5 & 2 \\
\hline & & $27503-81-7$ & PBS (Phenylbenzimidazole sulfonic acid) & 7 & 2 \\
\hline & & $58-93-5$ & Hydrochlorothiazide & 5 & 2 \\
\hline & & $90357-06-5$ & bicalutamide & 3 & 2 \\
\hline
\end{tabular}


Table 14: Effluent Iceland

\begin{tabular}{|c|c|c|c|c|c|}
\hline $\begin{array}{l}\text { Quant } \\
\text { (ng/L) }\end{array}$ & $\begin{array}{l}\text { Molecular } \\
\text { Formula }\end{array}$ & CAS & Common name & $\begin{array}{l}\text { Occur/ } \\
\text { matrix }\end{array}$ & $\begin{array}{r}\text { ID } \\
\text { Level }\end{array}$ \\
\hline 32217,37 & $\mathrm{C} 8 \mathrm{H}_{8} \mathrm{O}_{2}$ & $582-24-1$ & Ethanone, 2-hydroxy-1-phenyl- & 7 & 2 \\
\hline 26445,96 & $\mathrm{C}_{8} \mathrm{H}_{10} \mathrm{~N}_{4} \mathrm{O}_{2}$ & $58-08-2$ & Caffeine & 7 & 3 \\
\hline 19931,70 & $\mathrm{C}_{12} \mathrm{H}_{12} \mathrm{O}_{5}$ & $5062-30-6$ & Phenacylidene diacetate & 1 & 2 \\
\hline 6967,67 & $\mathrm{C}_{14} \mathrm{H}_{14} \mathrm{O}$ & $19578-70-2$ & Benzene, 1-methyl-2-(phenylmethoxy)- & 5 & 2 \\
\hline 6129,79 & $\mathrm{C}_{8} \mathrm{H}_{10 O} \mathrm{O}_{2}$ & $122-99-6$ & Ethanol, 2-phenoxy- & 6 & 2 \\
\hline 5116,88 & $\mathrm{C}_{1} 9 \mathrm{H}_{4} 0 \mathrm{O}_{2} \mathrm{Si}$ & $55520-89-3$ & Hexadecanoic acid, trimethylsilyl ester & 7 & 2 \\
\hline 3939,27 & $\mathrm{C}_{10 \mathrm{H}_{22} \mathrm{O}_{3}}$ & $29911-28-2$ & 2-Propanol, 1-(2-butoxy-1-methylethoxy)- & 5 & 3 \\
\hline 3373,97 & $\mathrm{C}_{1} \mathrm{OH}_{2} \mathrm{OO}$ & $15356-70-4$ & DL-Menthol & 3 & 2 \\
\hline 2256,37 & $\mathrm{C}_{7} \mathrm{H}_{7} \mathrm{NO}_{2}$ & $21494-57-5$ & 1H-Pyrrole-2,5-dione, 3-ethenyl-4-methyl- & 1 & 3 \\
\hline 2186,77 & $\mathrm{C}_{8} \mathrm{H}_{10 O}$ & $60-12-8$ & Phenylethyl Alcohol & 2 & 3 \\
\hline 2175,35 & $\mathrm{C}_{1} \mathrm{OH}_{16}$ & $3760-14-3$ & 1,5-Cyclooctadiene, 1,5-dimethyl- & 7 & 3 \\
\hline 1118,58 & $\mathrm{C}_{10} \mathrm{H}_{22} \mathrm{O}$ & $78-69-3$ & 3-Octanol, 3,7-dimethyl- & 3 & 2 \\
\hline 732,87 & $\mathrm{C}_{12} \mathrm{H}_{24} \mathrm{O}_{3}$ & $74367-34-3$ & Propanoic acid, 2-methyl-, 3-hydroxy-2,4,4-trimethylpentyl ester & 7 & 2 \\
\hline 732,61 & $\mathrm{C}_{1} \mathrm{OH}_{12} \mathrm{O}_{2}$ & $122-84-9$ & 2-Propanone, 1-(4-methoxyphenyl)- & 1 & 2 \\
\hline 589,01 & $\mathrm{C}_{12} \mathrm{H}_{2} \mathrm{OO}_{2}$ & 18679-18-0 & $2(3 \mathrm{H})$-Furanone, dihydro-5-(2-octenyl)-, (Z)- & 1 & 2 \\
\hline 492,40 & $\mathrm{C} 6 \mathrm{H}_{6} \mathrm{O}_{2}$ & $108-46-3$ & Resorcinol & 1 & 2 \\
\hline 376,55 & $\mathrm{C}_{7} \mathrm{H}_{9} \mathrm{NO}_{2}$ & $20189-42-8$ & 1H-Pyrrole-2,5-dione, 3-ethyl-4-methyl- & 7 & 3 \\
\hline 357,81 & $\mathrm{C}_{17} \mathrm{H}_{3} 6 \mathrm{O}_{2} \mathrm{Si}$ & $18603-17-3$ & Tetradecanoic acid, trimethylsilyl ester & 5 & 2 \\
\hline 335,31 & $\mathrm{C}_{7} \mathrm{H}_{8} \mathrm{O}_{2}$ & $504-15-4$ & Orcinol & 1 & 2 \\
\hline 314,70 & $\mathrm{C}_{2} \mathrm{OH}_{32} \mathrm{O}_{2}$ & - & Benzoic acid, tridecyl ester & 7 & 3 \\
\hline 310,39 & $\mathrm{C}_{13} \mathrm{H}_{10} \mathrm{O}$ & $119-61-9$ & Benzophenone & 7 & 2 \\
\hline 278,29 & $\mathrm{C}_{1} \mathrm{OH} 8$ & $91-20-3$ & Naphthalene & 1 & 2 \\
\hline 277,94 & $\mathrm{C} 8 \mathrm{H}_{8} \mathrm{O}_{2}$ & $57295-30-4$ & 3-Hydroxy-4-methylbenzaldehyde & 2 & 3 \\
\hline 271,63 & $\mathrm{C}_{1} 8 \mathrm{H}_{2} 8 \mathrm{O}_{2}$ & $6316-30-9$ & Benzoic acid, undecyl ester & 7 & 3 \\
\hline 265,96 & $\mathrm{C}_{1} 8 \mathrm{H}_{3} 8 \mathrm{O}_{2} \mathrm{Si}$ & $74367-22-9$ & n-Pentadecanoic acid, trimethylsilyl ester & 4 & 2 \\
\hline 249,69 & $\mathrm{C}_{10 \mathrm{H}_{14}}$ & $527-53-7$ & Benzene, 1,2,3,5-tetramethyl- & 1 & 2 \\
\hline 225,38 & $\mathrm{C}_{9} \mathrm{H}_{14} \mathrm{O} 6$ & $102-76-1$ & Triacetin & 4 & 2 \\
\hline 213,52 & $\mathrm{C}_{10 \mathrm{H}_{14} \mathrm{O}}$ & $39852-87-4$ & Tricyclo[5-2.1.o(2,6)]dec-3-en-10-ol & 2 & 3 \\
\hline 195,83 & $\mathrm{C}_{10 \mathrm{H}_{22} \mathrm{O}_{2}}$ & $1559-35-9$ & Ethanol, 2-[(2-ethylhexyl)oxy]- & 1 & 2 \\
\hline 193,41 & $\mathrm{C}_{22} \mathrm{H}_{3} 6 \mathrm{O}_{2}$ & - & Benzoic acid, pentadecyl ester & 6 & 3 \\
\hline 187,42 & $\mathrm{C}_{8} \mathrm{H}_{7} \mathrm{NO}$ & $2380-94-1$ & 1H-Indol-4-ol & 2 & 3 \\
\hline 172,97 & 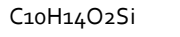 & $2078-12-8$ & Benzoic acid trimethylsilyl ester & 1 & 2 \\
\hline 162,88 & $\mathrm{C}_{10 \mathrm{H}_{14} \mathrm{O}}$ & $99-49-0$ & Carvone & 5 & 2 \\
\hline 161,99 & $\mathrm{C}_{8} \mathrm{H}_{14} \mathrm{O}_{4}$ & $627-93-0$ & Hexanedioic acid, dimethyl ester & 5 & 3 \\
\hline 155,73 & $\mathrm{C}_{10 \mathrm{H}_{14}}$ & $488-23-3$ & Benzene, 1,2,3,4-tetramethyl- & 1 & 2 \\
\hline 153,53 & $\mathrm{C}_{9} \mathrm{H}_{10} \mathrm{O}_{2}$ & $140-11-4$ & Acetic acid, phenylmethyl ester & 1 & 2 \\
\hline 142,14 & $\mathrm{C}_{21} \mathrm{H}_{44} \mathrm{O}_{2} \mathrm{Si}$ & 18748-91-9 & Octadecanoic acid, trimethylsilyl ester & 4 & 2 \\
\hline 102,41 & $\mathrm{C}_{8} \mathrm{H}_{7} \mathrm{NS}_{2}$ & $615-22-5$ & Benzothiazole, 2-(methylthio)- & 5 & 2 \\
\hline 81,38 & $\mathrm{C}_{15} \mathrm{H}_{13} \mathrm{~N}$ & $2286-54-6$ & Benzenepropanenitrile, á-phenyl- & 5 & 2 \\
\hline 79,00 & $\mathrm{C}_{9} \mathrm{H}_{6} \mathrm{O}_{2}$ & $91-64-5$ & Coumarin & 3 & 2 \\
\hline 62,66 & $\mathrm{C}_{13} \mathrm{H}_{12}$ & $643-93-6$ & 1,1'-Biphenyl, 3-methyl- & 1 & 2 \\
\hline 58,71 & $\mathrm{C}_{5} \mathrm{H}_{9} \mathrm{NO}_{2}$ & $4394-85-8$ & N-Formylmorpholine & 3 & 2 \\
\hline \multirow[t]{21}{*}{43,71} & $\mathrm{C}_{11} \mathrm{H}_{10}$ & $90-12-0$ & Naphthalene, 1-methyl- & 2 & 2 \\
\hline & $\mathrm{C}_{2} \mathrm{OH}_{23} \mathrm{~N}$ & & Amitriptyline & 6 & 2 \\
\hline & $\mathrm{C}_{2} \mathrm{OH}_{25} \mathrm{ClN}_{2} \mathrm{O}_{5}$ & & Amlodipine & 6 & 2 \\
\hline & $\mathrm{C}_{14} \mathrm{H}_{22} \mathrm{~N}_{2} \mathrm{O}_{3}$ & & Atenolol & 7 & 2 \\
\hline & $\mathrm{C}_{3} 8 \mathrm{H}_{72} \mathrm{~N}_{2} \mathrm{O}_{12}$ & & Azithromycin & 6 & 2 \\
\hline & $\mathrm{C}_{1} 6 \mathrm{H}_{19} \mathrm{NO}_{4}$ & & Benzoylecgonine & 5 & 2 \\
\hline & $\mathrm{C}_{8} \mathrm{H}_{10} \mathrm{~N}_{4} \mathrm{O}_{2}$ & & Caffeine & 7 & 2 \\
\hline & $\mathrm{C}_{15} \mathrm{H}_{12} \mathrm{~N}_{2} \mathrm{O}$ & & Carbamazepine & 7 & 2 \\
\hline & $\mathrm{C}_{15} \mathrm{H}_{14} \mathrm{~N}_{2} \mathrm{O}_{3}$ & & Carbamazepine, 10, 11-dihydroxy & 7 & 2 \\
\hline & $\mathrm{C}_{15} \mathrm{H}_{14} \mathrm{~N}_{2} \mathrm{O}_{2}$ & & Carbamazepine, 10-hydroxy & 7 & 2 \\
\hline & $\mathrm{C}_{21} \mathrm{H}_{25} \mathrm{ClN}_{2} \mathrm{O}_{3}$ & & Cetirizine & 7 & 2 \\
\hline & $\mathrm{C}_{17} \mathrm{H}_{18} 8 \mathrm{FN}_{3} \mathrm{O}_{3}$ & & Ciprofloxacin & 7 & 2 \\
\hline & $\mathrm{C}_{2} \mathrm{OH}_{21} \mathrm{FN} 2 \mathrm{O}$ & & Citalopram/Escitalopram & 7 & 2 \\
\hline & $\mathrm{C}_{3} 8 \mathrm{H}_{69} \mathrm{NO}_{13}$ & & Clarithromycin & 6 & 2 \\
\hline & $\mathrm{C}_{18} \mathrm{H}_{19} \mathrm{ClN}_{4}$ & & Clozapine & 7 & 2 \\
\hline & $\mathrm{C}_{17} \mathrm{H}_{17} \mathrm{ClN}_{4}$ & & Clozapine, Desmethyl & 6 & 2 \\
\hline & $\mathrm{C}_{17} \mathrm{H}_{21} \mathrm{NO}_{4}$ & & Cocaine & 5 & 2 \\
\hline & $\mathrm{C}_{1} 8 \mathrm{H}_{21} \mathrm{NO}_{3}$ & & Codeine & 6 & 2 \\
\hline & $\mathrm{C}_{1} \mathrm{OH}_{12} \mathrm{~N}_{2} \mathrm{O}$ & & Cotinine & 7 & 2 \\
\hline & $\mathrm{C}_{22} \mathrm{H}_{2} 6 \mathrm{~N}_{2} \mathrm{O}_{4} \mathrm{~S}$ & & Diltiazem & 6 & 2 \\
\hline & $\mathrm{C}_{24} \mathrm{H}_{4} \mathrm{ON} 8 \mathrm{O}_{4}$ & & Dipyridamol & 4 & 2 \\
\hline
\end{tabular}


Continued

\begin{tabular}{|c|c|c|c|c|c|}
\hline $\begin{array}{l}\text { Quant } \\
\text { (ng/L) }\end{array}$ & $\begin{array}{l}\text { Molecular } \\
\text { Formula }\end{array}$ & CAS & Common name & $\begin{array}{l}\text { Occur/ } \\
\text { matrix }\end{array}$ & $\begin{array}{r}\text { ID } \\
\text { Level }\end{array}$ \\
\hline & $\mathrm{C}_{32} \mathrm{H}_{39} \mathrm{NO}_{4}$ & & Fexofenadine & 7 & 2 \\
\hline & $\mathrm{C}_{17} \mathrm{H}_{2} \mathrm{O} \mathrm{F}_{6 \mathrm{~N}_{2} \mathrm{O}_{3}}$ & & Flecainide & 5 & 2 \\
\hline & $\mathrm{C}_{1} 8 \mathrm{H}_{21} \mathrm{NO}_{3}$ & & Hydrocodone & 6 & 2 \\
\hline & $\mathrm{C}_{25} \mathrm{H}_{28} 8 \mathrm{~N} 6 \mathrm{O}$ & & Irbesartan & 4 & 2 \\
\hline & $\mathrm{C}_{2} 6 \mathrm{H}_{2} 8 \mathrm{Cl}_{2} \mathrm{~N}_{4} \mathrm{O}_{4}$ & & Ketoconazole & 7 & 2 \\
\hline & $\mathrm{C}_{9} \mathrm{H}_{7} \mathrm{Cl}_{2} \mathrm{~N}_{5}$ & & Lamotrigine & 7 & 2 \\
\hline & $\mathrm{C}_{22} \mathrm{H}_{23} \mathrm{ClN} 6 \mathrm{O}$ & & Losartan & 7 & 2 \\
\hline & $\mathrm{C}_{2} \mathrm{OH}_{23} \mathrm{~N}$ & & Maprotiline & 6 & 2 \\
\hline & $\mathrm{C}_{11} \mathrm{H}_{15} \mathrm{NO}_{2}$ & & MDMA & 5 & 2 \\
\hline & $\mathrm{C}_{14} \mathrm{H}_{22} \mathrm{ClN}_{3} \mathrm{O}_{2}$ & & Metoclopramide & 6 & 2 \\
\hline & $\mathrm{C}_{15} \mathrm{H}_{25} \mathrm{NO}_{3}$ & & Metoprolol & 7 & 2 \\
\hline & $\mathrm{C}_{17} \mathrm{H}_{19} \mathrm{~N}_{3}$ & & Mirtazapine & 7 & 2 \\
\hline & 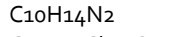 & & Nicotine & 7 & 2 \\
\hline & $\mathrm{C}_{15} \mathrm{H}_{11} \mathrm{ClN}_{2} \mathrm{O}_{2}$ & & Oxazepam & 6 & 2 \\
\hline & $\mathrm{C}_{1} 6 \mathrm{H}_{21} \mathrm{NO}_{2}$ & & Propranolol & 7 & 2 \\
\hline & $\mathrm{C}_{21} \mathrm{H}_{25} \mathrm{~N}_{3} \mathrm{O}_{2} \mathrm{~S}$ & & Quetiapine & 7 & 2 \\
\hline & $\mathrm{C}_{2} \mathrm{OH}_{24} \mathrm{~N}_{2} \mathrm{O}_{2}$ & & Quinine & 7 & 2 \\
\hline & $\mathrm{C}_{1} 6 \mathrm{H}_{15} \mathrm{~F}_{6} \mathrm{~N}_{5} \mathrm{O}$ & & Sitagliptin & 5 & 2 \\
\hline & $\mathrm{C}_{12} \mathrm{H}_{2} \mathrm{ON}_{2} \mathrm{O}_{3} \mathrm{~S}$ & & Sotalol & 6 & 2 \\
\hline & $\mathrm{C}_{11} \mathrm{H}_{11} \mathrm{~N}_{3} \mathrm{O}_{2} \mathrm{~S}$ & & Sulfapyridine & 8 & 2 \\
\hline & $\mathrm{C}_{14} \mathrm{H}_{21} \mathrm{~N}_{3} \mathrm{O}_{2} \mathrm{~S}$ & & Sumatriptan & 9 & 2 \\
\hline & $\mathrm{C}_{33} \mathrm{H}_{3} \mathrm{ON}_{4} \mathrm{O}_{2}$ & & Telmisartan & 5 & 2 \\
\hline & $\mathrm{C}_{1} 6 \mathrm{H}_{2}{ }_{5} \mathrm{NO}_{2}$ & & Tramadol & 7 & 2 \\
\hline & $\mathrm{C}_{14} \mathrm{H}_{1} 8 \mathrm{~N}_{4} \mathrm{O}_{3}$ & & Trimethoprim & 7 & 2 \\
\hline & $\mathrm{C}_{24} \mathrm{H}_{29} \mathrm{~N}_{5} \mathrm{O}_{3}$ & & Valsartan & 5 & 2 \\
\hline & $\mathrm{C}_{17} \mathrm{H}_{27} \mathrm{NO}_{2}$ & & Venlafaxine & 7 & 2 \\
\hline & $\mathrm{C}_{1} 6 \mathrm{H}_{25} \mathrm{NO}_{2}$ & & Venlafaxine,-O-demethyl- & 7 & 2 \\
\hline & $\mathrm{C}_{15} \mathrm{H}_{11} \mathrm{ClO}_{3}$ & $2536-31-4$ & Chlorflurenol-methyl (ester) & 2 & 2 \\
\hline & $\mathrm{C}_{6} \mathrm{H}_{12} \mathrm{O} 6$ & $50-99-7$ & glucose & 1 & 2 \\
\hline & $\mathrm{C}_{2} \mathrm{OH}_{4} \mathrm{OOO}_{2}$ & $506-30-9$ & icosanoic acid & 2 & 2 \\
\hline & $\mathrm{C}_{29} \mathrm{H}_{44} \mathrm{O}_{12}$ & $630-60-4$ & ouabain & 1 & 2 \\
\hline & $\mathrm{C}_{17} \mathrm{H}_{34} \mathrm{O}_{2}$ & $6309-51-9$ & isopentyl laurate & 3 & 2 \\
\hline & $\mathrm{C}_{17} \mathrm{H}_{34} \mathrm{O}_{2}$ & $110-27-0$ & Wickenol 101 & 3 & 2 \\
\hline & $\mathrm{C}_{17} \mathrm{H}_{34} \mathrm{O}_{2}$ & $112-39-0$ & methyl palmitate & 2 & 2 \\
\hline & $\mathrm{C}_{1} 6 \mathrm{H}_{3} \mathrm{OO}_{2}$ & $142-90-5$ & dodecyl methacrylate & 2 & 2 \\
\hline & $\mathrm{C}_{1} 8 \mathrm{H}_{3} 6 \mathrm{O}_{2}$ & $57-11-4$ & Octadecanoic acid & 2 & 2 \\
\hline & $\mathrm{C}_{1} 6 \mathrm{H}_{10} \mathrm{ClN}_{3} \mathrm{O}_{3}$ & $2814-77-9$ & 1-[(2-chloro-4-nitrophenyl)azo]-2-naphthol & 2 & 2 \\
\hline & & $80-09-1$ & bisphenol S & 3 & 2 \\
\hline & & $620-92-8$ & 4,4-BPF & 4 & 1 \\
\hline & & $13595-25-0$ & bisphenol M & 5 & 2 \\
\hline & & $123-08-0$ & 4-hydroxybenzaldehyde & 7 & 2 \\
\hline & & $1074-86-8$ & 4-formyl indole & 6 & 2 \\
\hline & & $137862-53-4$ & Valsartan & 3 & 2 \\
\hline & & $93-35-6$ & umbelliferone & 1 & 2 \\
\hline & & $58-93-5$ & Hydrochlorothiazide & 5 & 2 \\
\hline & & $103-90-2$ & paracetamol & 5 & 2 \\
\hline
\end{tabular}


Table 15: Effluent Norway

\begin{tabular}{|c|c|c|c|c|c|}
\hline $\begin{array}{l}\text { Quant } \\
\text { (ng/L) }\end{array}$ & $\begin{array}{l}\text { Molecular } \\
\text { Formula }\end{array}$ & CAS & Common name & $\begin{array}{l}\text { Occur/ } \\
\text { matrix }\end{array}$ & $\begin{array}{l}\text { ID } \\
\text { Level }\end{array}$ \\
\hline 2448,71 & $\mathrm{C}_{1} \mathrm{OH}_{22} \mathrm{O}_{3}$ & 29911-28-2 & 2-Propanol, 1-(2-butoxy-1-methylethoxy)- & 5 & 2 \\
\hline 1826,70 & $\mathrm{C}_{18} \mathrm{H}_{39} \mathrm{O}_{7} \mathrm{P}$ & $78-51-3$ & Ethanol, 2-butoxy-, phosphate (3:1) & 2 & 2 \\
\hline 825,94 & $\mathrm{C}_{10 \mathrm{H}_{1} 6}$ & $3760-14-3$ & 1,5-Cyclooctadiene, 1,5-dimethyl- & 7 & 2 \\
\hline 644,57 & $\mathrm{C}_{19} \mathrm{H}_{4} \mathrm{OO} 2 \mathrm{Si}$ & $55520-89-3$ & Hexadecanoic acid, trimethylsilyl ester & 7 & 2 \\
\hline 541,62 & $\mathrm{C}_{8} \mathrm{H}_{10} \mathrm{~N}_{4} \mathrm{O}_{2}$ & $58-08-2$ & Caffeine & 7 & 2 \\
\hline 338,05 & $\mathrm{C}_{1} 8 \mathrm{H}_{2} 6 \mathrm{O}$ & $1222-05-5$ & Galaxolide & 6 & 2 \\
\hline 335,23 & $\mathrm{C}_{7} \mathrm{H}_{9} \mathrm{NO}_{2}$ & $20189-42-8$ & 1H-Pyrrole-2,5-dione, 3-ethyl-4-methyl- & 7 & 2 \\
\hline 242,90 & $\mathrm{C}_{13} \mathrm{H}_{10 O}$ & $119-61-9$ & Benzophenone & 7 & 2 \\
\hline 213,55 & $\mathrm{C} 8 \mathrm{H}_{8} \mathrm{O}_{2}$ & $582-24-1$ & Ethanone, 2-hydroxy-1-phenyl- & 7 & 2 \\
\hline 194,79 & $\mathrm{C} 8 \mathrm{H}_{6} \mathrm{O}_{2}$ & $1074-12-0$ & Phenylglyoxal & 6 & 3 \\
\hline 192,41 & $\mathrm{C}_{14} \mathrm{H}_{11} \mathrm{~N}$ & $611-64-3$ & Acridine, 9-methyl- & 5 & 2 \\
\hline 134,97 & $\mathrm{C}_{21} \mathrm{H}_{44} \mathrm{O}_{2} \mathrm{Si}$ & 18748-91-9 & Octadecanoic acid, trimethylsilyl ester & 4 & 2 \\
\hline 132,98 & $\mathrm{C}_{14} \mathrm{H}_{14} \mathrm{O}$ & $19578-70-2$ & Benzene, 1-methyl-2-(phenylmethoxy)- & 5 & 3 \\
\hline 113,72 & $\mathrm{C}_{9} \mathrm{H}_{14} \mathrm{O} 6$ & $102-76-1$ & Triacetin & 4 & 3 \\
\hline 95,44 & $\mathrm{C}_{1} 8 \mathrm{H}_{2} 8 \mathrm{O}_{2}$ & $6316-30-9$ & Benzoic acid, undecyl ester & 7 & 3 \\
\hline 89,52 & $\mathrm{C}_{2} \mathrm{OH}_{32} \mathrm{O}_{2}$ & - & Benzoic acid, tridecyl ester & 7 & 3 \\
\hline 80,03 & $\mathrm{C}_{15} \mathrm{H}_{13} \mathrm{~N}$ & $2286-54-6$ & Benzenepropanenitrile, á-phenyl- & 5 & 2 \\
\hline 77,63 & $\mathrm{C}_{2} 2 \mathrm{H}_{3} 6 \mathrm{O}_{2}$ & - & Benzoic acid, pentadecyl ester & 6 & 2 \\
\hline 67,64 & $\mathrm{C}_{1} \mathrm{OH}_{15} \mathrm{NO}_{2} \mathrm{~S}$ & $3622-84-2$ & Benzenesulfonamide, N-butyl- & 3 & 2 \\
\hline 65,53 & $\mathrm{C}_{12} \mathrm{H}_{24} \mathrm{O}_{3}$ & $74367-34-3$ & Propanoic acid, 2-methyl-, 3-hydroxy-2,4,4-trimethylpentyl ester & 7 & 3 \\
\hline 61,76 & $\mathrm{C}_{6} \mathrm{H}_{7} \mathrm{NS}$ & $18438-38-5$ & Pyridine, 2-(methylthio)- & 4 & 2 \\
\hline 50,79 & $\mathrm{C}_{8} \mathrm{H}_{14} \mathrm{O}_{4}$ & $627-93-0$ & Hexanedioic acid, dimethyl ester & 5 & 2 \\
\hline 49,39 & $\mathrm{C}_{7} \mathrm{H} 8 \mathrm{O}$ & $108-39-4$ & Phenol, 3-methyl- & 3 & 3 \\
\hline 40,21 & $\mathrm{C}_{15} \mathrm{H}_{11} \mathrm{~N}$ & $3531-24-6$ & 2-Propenenitrile, 3,3-diphenyl- & 3 & 3 \\
\hline \multirow[t]{39}{*}{34,02} & $\mathrm{C} 8 \mathrm{H}_{8} \mathrm{O}_{2}$ & $57295-30-4$ & 3-Hydroxy-4-methylbenzaldehyde & 2 & 3 \\
\hline & $\mathrm{C}_{1} 8 \mathrm{H}_{2} 8 \mathrm{~N}_{2} \mathrm{O}_{4}$ & & Acebutolol & 3 & 2 \\
\hline & $\mathrm{C}_{2} \mathrm{OH}_{23} \mathrm{~N}$ & & Amitriptyline & 6 & 2 \\
\hline & $\mathrm{C}_{3} 8 \mathrm{H}_{52} \mathrm{~N}_{6} \mathrm{O}_{7}$ & & Atazanavir & 5 & 2 \\
\hline & $\mathrm{C}_{14} \mathrm{H}_{22} \mathrm{~N}_{2} \mathrm{O}_{3}$ & & Atenolol & 7 & 2 \\
\hline & $\mathrm{C}_{3} 8 \mathrm{H}_{72} \mathrm{~N}_{2} \mathrm{O}_{12}$ & & Azithromycin & 6 & 2 \\
\hline & $\mathrm{C}_{1} 6 \mathrm{H}_{19} \mathrm{NO}_{4}$ & & Benzoylecgonine & 5 & 2 \\
\hline & $\mathrm{C}_{1} 8 \mathrm{H}_{31} \mathrm{NO}_{4}$ & & Bisoprolol & 4 & 2 \\
\hline & $\mathrm{C}_{8} \mathrm{H}_{10} \mathrm{~N}_{4} \mathrm{O}_{2}$ & & Caffeine & 7 & 2 \\
\hline & $\mathrm{C}_{15} \mathrm{H}_{12} \mathrm{~N}_{2} \mathrm{O}$ & & Carbamazepine & 7 & 2 \\
\hline & $\mathrm{C}_{15} \mathrm{H}_{14} \mathrm{~N}_{2} \mathrm{O}_{3}$ & & Carbamazepine, 10, 11-dihydroxy & 7 & 2 \\
\hline & $\mathrm{C}_{15} \mathrm{H}_{14} \mathrm{~N}_{2} \mathrm{O}_{2}$ & & Carbamazepine, 10-hydroxy & 7 & 2 \\
\hline & $\mathrm{C}_{2} \mathrm{OH}_{3} \mathrm{~N}_{3} \mathrm{O}_{4}$ & & Celiprolol & 4 & 2 \\
\hline & $\mathrm{C}_{21} \mathrm{H}_{25} \mathrm{ClN}_{2} \mathrm{O}_{3}$ & & Cetirizine & 7 & 2 \\
\hline & $\mathrm{C}_{17} \mathrm{H}_{18} \mathrm{FN}_{3} \mathrm{O}_{3}$ & & Ciprofloxacin & 7 & 2 \\
\hline & $\mathrm{C}_{2} \mathrm{OH}_{21} \mathrm{FN}_{2} \mathrm{O}$ & & Citalopram/Escitalopram & 7 & 2 \\
\hline & $\mathrm{C}_{3} 8 \mathrm{H}_{69} \mathrm{NO}_{13}$ & & Clarithromycin & 6 & 2 \\
\hline & $\mathrm{C}_{18} \mathrm{H}_{19} \mathrm{ClN}_{4}$ & & Clozapine & 7 & 2 \\
\hline & $\mathrm{C}_{17} \mathrm{H}_{17} \mathrm{ClN}_{4}$ & & Clozapine, Desmethyl & 6 & 2 \\
\hline & $\mathrm{C}_{17} \mathrm{H}_{21} \mathrm{NO}_{4}$ & & Cocaine & 5 & 2 \\
\hline & $\mathrm{C}_{1} 8 \mathrm{H}_{21} \mathrm{NO}_{3}$ & & Codeine & 6 & 2 \\
\hline & $\mathrm{C}_{1} \mathrm{OH}_{12} \mathrm{~N}_{2} \mathrm{O}$ & & Cotinine & 7 & 2 \\
\hline & $\mathrm{C}_{22} \mathrm{H}_{2} 6 \mathrm{~N}_{2} \mathrm{O}_{4} \mathrm{~S}$ & & Diltiazem & 6 & 2 \\
\hline & $\mathrm{C}_{2} 4 \mathrm{H}_{4} \mathrm{ON} 8 \mathrm{O}_{4}$ & & Dipyridamol & 4 & 2 \\
\hline & $\mathrm{C}_{23} \mathrm{H}_{24} \mathrm{~N}_{2} \mathrm{O}_{4} \mathrm{~S}$ & & Eprosartan & 3 & 2 \\
\hline & $\mathrm{C}_{3} \mathrm{H}_{39} \mathrm{NO}_{4}$ & & Fexofenadine & 7 & 2 \\
\hline & $\mathrm{C}_{17} \mathrm{H}_{2} \mathrm{OF} 6 \mathrm{~N}_{2} \mathrm{O}_{3}$ & & Flecainide & 5 & 2 \\
\hline & $\mathrm{C}_{1} 8 \mathrm{H}_{21} \mathrm{NO}_{3}$ & & Hydrocodone & 6 & 2 \\
\hline & $\mathrm{C}_{25} \mathrm{H}_{28} \mathrm{~N} 6 \mathrm{O}$ & & Irbesartan & 4 & 2 \\
\hline & $\mathrm{C}_{2} 6 \mathrm{H}_{2} 8 \mathrm{Cl}_{2} \mathrm{~N}_{4} \mathrm{O}_{4}$ & & Ketoconazole & 7 & 2 \\
\hline & $\mathrm{C}_{9} \mathrm{H}_{7} \mathrm{Cl}_{2} \mathrm{~N}_{5}$ & & Lamotrigine & 7 & 2 \\
\hline & $\mathrm{C}_{22} \mathrm{H}_{23} \mathrm{ClN} 6 \mathrm{O}$ & & Losartan & 7 & 2 \\
\hline & $\mathrm{C}_{2} \mathrm{OH}_{23} \mathrm{~N}$ & & Maprotiline & 6 & 2 \\
\hline & $\mathrm{C}_{11} \mathrm{H}_{15} \mathrm{NO}_{2}$ & & MDMA & 5 & 2 \\
\hline & $\mathrm{C}_{21} \mathrm{H}_{27} \mathrm{NO}$ & & Methadone & 4 & 2 \\
\hline & $\mathrm{C}_{14} \mathrm{H}_{22} \mathrm{ClN}_{3} \mathrm{O}_{2}$ & & Metoclopramide & 6 & 2 \\
\hline & $\mathrm{C}_{15} \mathrm{H}_{25} \mathrm{NO}_{3}$ & & Metoprolol & 7 & 2 \\
\hline & $\mathrm{C}_{17} \mathrm{H}_{19} \mathrm{~N}_{3}$ & & Mirtazapine & 7 & 2 \\
\hline & 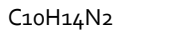 & & Nicotine & 7 & 2 \\
\hline
\end{tabular}


Continued

\begin{tabular}{|c|c|c|c|c|c|}
\hline $\begin{array}{l}\text { Quant } \\
\text { (ng/L) }\end{array}$ & $\begin{array}{l}\text { Molecular } \\
\text { Formula }\end{array}$ & CAS & Common name & $\begin{array}{l}\text { Occur/ } \\
\text { matrix }\end{array}$ & $\begin{array}{r}\text { ID } \\
\text { Level }\end{array}$ \\
\hline & $\mathrm{C}_{15} \mathrm{H}_{11} \mathrm{ClN}_{2} \mathrm{O}_{2}$ & & Oxazepam & 6 & 2 \\
\hline & $\mathrm{C}_{1} 6 \mathrm{H}_{21} \mathrm{NO}_{2}$ & & Propranolol & 7 & 2 \\
\hline & $\mathrm{C}_{21} \mathrm{H}_{25} \mathrm{~N}_{3} \mathrm{O}_{2} \mathrm{~S}$ & & Quetiapine & 7 & 2 \\
\hline & $\mathrm{C}_{2} \mathrm{OH}_{2}{ }_{4} \mathrm{~N}_{2} \mathrm{O}_{2}$ & & Quinine & 7 & 2 \\
\hline & $\mathrm{C}_{1} 6 \mathrm{H}_{15} \mathrm{~F}_{6} \mathrm{~N}_{5} \mathrm{O}$ & & Sitagliptin & 5 & 2 \\
\hline & $\mathrm{C}_{12} \mathrm{H}_{2} \mathrm{ON}_{2} \mathrm{O}_{3} \mathrm{~S}$ & & Sotalol & 6 & 2 \\
\hline & $\mathrm{C}_{11} \mathrm{H}_{11} \mathrm{~N}_{3} \mathrm{O}_{2} \mathrm{~S}$ & & Sulfapyridine & 7 & 2 \\
\hline & $\mathrm{C}_{15} \mathrm{H}_{23} \mathrm{~N}_{3} \mathrm{O}_{4} \mathrm{~S}$ & & Sulpiride & 4 & 2 \\
\hline & $\mathrm{C}_{14} \mathrm{H}_{21} \mathrm{~N}_{3} \mathrm{O}_{2} \mathrm{~S}$ & & Sumatriptan & 7 & 2 \\
\hline & $\mathrm{C}_{33} \mathrm{H}_{3} \mathrm{~N}_{4} \mathrm{O}_{2}$ & & Telmisartan & 5 & 2 \\
\hline & $\mathrm{C}_{1} 6 \mathrm{H}_{25} \mathrm{NO}_{2}$ & & Tramadol & 7 & 2 \\
\hline & $\mathrm{C}_{14} \mathrm{H}_{1} 8 \mathrm{~N}_{4} \mathrm{O}_{3}$ & & Trimethoprim & 7 & 2 \\
\hline & $\mathrm{C}_{24} \mathrm{H}_{29} \mathrm{~N}_{5} \mathrm{O}_{3}$ & & Valsartan & 5 & 2 \\
\hline & $\mathrm{C}_{17} \mathrm{H}_{27} \mathrm{NO}_{2}$ & & Venlafaxine & 7 & 2 \\
\hline & $\mathrm{C}_{1} 6 \mathrm{H}_{25} \mathrm{NO}_{2}$ & & Venlafaxine,-O-demethyl- & 7 & 2 \\
\hline & $\mathrm{C}_{4} \mathrm{H}_{4} \mathrm{~N}_{2} \mathrm{O}_{3}$ & $67-52-7$ & $2,4,6(1 \mathrm{H}, 3 \mathrm{H}, 5 \mathrm{H})$-Pyrimidinetrione & 1 & 2 \\
\hline & $\mathrm{C}_{5} \mathrm{H}_{8} \mathrm{O}_{3}$ & $105-45-3$ & methyl acetoacetate & 2 & 2 \\
\hline & $\mathrm{C}_{17} \mathrm{H}_{34} \mathrm{O}_{2}$ & $6309-51-9$ & isopentyl laurate & 3 & 2 \\
\hline & $\mathrm{C}_{17} \mathrm{H}_{34} \mathrm{O}_{2}$ & $110-27-0$ & Wickenol 101 & 3 & 2 \\
\hline & $\mathrm{C}_{15} \mathrm{H}_{3} \mathrm{OO}_{2}$ & $124-10-7$ & methyl myristate & 1 & 2 \\
\hline & $\mathrm{C}_{15} \mathrm{H}_{3} \mathrm{OO}_{2}$ & $10233-13-3$ & isopropyl laurate & 1 & 2 \\
\hline & $\mathrm{C}_{14} \mathrm{H}_{2} 8 \mathrm{O}_{2}$ & $544-63-8$ & Myristic acid & 1 & 2 \\
\hline & $\mathrm{C}_{15} \mathrm{H}_{2} 8 \mathrm{O}_{2}$ & $106-02-5$ & pentadecan-15-olide & 1 & 2 \\
\hline & $\mathrm{C}_{7} \mathrm{H}_{5} \mathrm{NO}$ & $103-71-9$ & Phenylisocyanate & 1 & 2 \\
\hline & $\mathrm{C}_{15} \mathrm{H}_{11} \mathrm{ClO}_{3}$ & $2536-31-4$ & Chlorflurenol-methyl (ester) & 2 & 2 \\
\hline & $\mathrm{C}_{1} 6 \mathrm{H}_{10} \mathrm{ClN}_{3} \mathrm{O}_{3}$ & $2814-77-9$ & 1-[(2-chloro-4-nitrophenyl)azo]-2-naphthol & 2 & 2 \\
\hline & $\mathrm{C}_{1} 6 \mathrm{H}_{32} \mathrm{O}_{2}$ & $2306-88-9$ & octyl octanoate & 2 & 2 \\
\hline & $\mathrm{C}_{1} 6 \mathrm{H}_{32} \mathrm{O}_{2}$ & $57-10-3$ & Hexadecanoic acid & 2 & 2 \\
\hline & & $1799-84-4$ & 4:2 FTMAC & 5 & 2 \\
\hline & & $68259-15-4$ & MeFHxSA & 2 & 2 \\
\hline & & $80-09-1$ & bisphenol S & 3 & 1 \\
\hline & & $620-92-8$ & $4,4-B P F$ & 4 & 1 \\
\hline & & $13595-25-0$ & bisphenol M & 5 & 2 \\
\hline & & $123-08-0$ & 4-hydroxybenzaldehyde & 7 & 2 \\
\hline & & $1074-86-8$ & 4-formyl indole & 6 & 2 \\
\hline & & $137862-53-4$ & Valsartan & 3 & 2 \\
\hline & & $4065-45-6$ & benzophenone-4 (sulisobenzone) & 5 & 2 \\
\hline & & $27503-81-7$ & PBS (Phenylbenzimidazole sulfonic acid) & 7 & 2 \\
\hline
\end{tabular}


Table 16: Effluent Greenland

\begin{tabular}{|c|c|c|c|c|c|}
\hline $\begin{array}{l}\text { Quant } \\
\text { (ng/L) }\end{array}$ & $\begin{array}{l}\text { Molecular } \\
\text { Formula }\end{array}$ & CAS & Common name & $\begin{array}{l}\text { Occur/ } \\
\text { matrix }\end{array}$ & $\begin{array}{r}\text { ID } \\
\text { Level }\end{array}$ \\
\hline 52627,52 & $\mathrm{C}_{8} \mathrm{H}_{10 O}$ & $122-99-6$ & Ethanol, 2-phenoxy- & 6 & 2 \\
\hline 26654,10 & $\mathrm{C}_{8} \mathrm{H}_{10} \mathrm{~N}_{4} \mathrm{O}_{2}$ & $58-08-2$ & Caffeine & 7 & 3 \\
\hline 14634,51 & $\mathrm{C}_{8} \mathrm{H}_{7} \mathrm{~N}$ & $120-72-9$ & Indole & 1 & 2 \\
\hline 11227,99 & $\mathrm{C}_{23} \mathrm{H}_{3} 6 \mathrm{O}_{4}$ & - & Phthalic acid, hept-3-yl octyl ester & 7 & 2 \\
\hline 6470,04 & $\mathrm{C}_{10} \mathrm{H}_{16}$ & $3760-14-3$ & 1,5-Cyclooctadiene, 1,5-dimethyl- & 7 & 3 \\
\hline 6194,59 & $\mathrm{C}_{1} \mathrm{oH} 2 \mathrm{OO}$ & $15356-70-4$ & DL-Menthol & 3 & 2 \\
\hline 5603,52 & $\mathrm{C}_{2} 4 \mathrm{H}_{3} 8 \mathrm{O}_{4}$ & $6422-86-2$ & 1,4-Benzenedicarboxylic acid, bis(2-ethylhexyl) ester & 1 & 3 \\
\hline 5546,29 & $\mathrm{C}_{8} \mathrm{H}_{10 O}$ & $60-12-8$ & Phenylethyl Alcohol & 2 & 3 \\
\hline 4470,41 & $\mathrm{C}_{10 H} \mathrm{H}_{22} \mathrm{O}$ & $78-69-3$ & 3-Octanol, 3,7-dimethyl- & 3 & 2 \\
\hline 3840,42 & $\mathrm{C}_{1} 6 \mathrm{H}_{34} \mathrm{O}_{3}$ & $3055-93-4$ & Diethylene glycol monododecyl ether & 1 & 2 \\
\hline 2506,84 & $\mathrm{C}_{1} 8 \mathrm{H}_{2} 6 \mathrm{O}$ & $1222-05-5$ & Galaxolide & 6 & 2 \\
\hline 2073,93 & $\mathrm{C}_{11} \mathrm{H}_{20 O_{2}}$ & $104-67-6$ & 2(3H)-Furanone, 5-heptyldihydro- & 1 & 2 \\
\hline 1955,10 & $\mathrm{C}_{14} \mathrm{H}_{2} 6 \mathrm{O}_{2}$ & $544-64-9$ & Myristoleic acid & 1 & 2 \\
\hline 1897,06 & $\mathrm{C}_{12} \mathrm{H}_{24} \mathrm{O}_{3}$ & $74367-34-3$ & Propanoic acid, 2-methyl-, 3-hydroxy-2,4,4-trimethylpentyl ester & 7 & 2 \\
\hline 1693,75 & $\mathrm{Cg}_{9} \mathrm{H} N$ & $83-34-1$ & 1H-Indole, 3-methyl- & 2 & 2 \\
\hline 1562,43 & $\mathrm{C}_{2} \mathrm{OH}_{32} \mathrm{O}_{2}$ & - & Benzoic acid, tridecyl ester & 7 & 3 \\
\hline 1415,24 & $\mathrm{C}_{17} \mathrm{H}_{3} 6 \mathrm{O}_{2} \mathrm{Si}$ & $18603-17-3$ & Tetradecanoic acid, trimethylsilyl ester & 5 & 2 \\
\hline 1271,36 & $\mathrm{C}_{1} 8 \mathrm{H}_{2} 8 \mathrm{O}_{2}$ & $6316-30-9$ & Benzoic acid, undecyl ester & 7 & 3 \\
\hline 1239,19 & $\mathrm{C}_{10} \mathrm{H}_{14} \mathrm{O}$ & $39852-87-4$ & Tricyclo[5-2.1.o(2,6)]dec-3-en-10-ol & 2 & 3 \\
\hline 1161,01 & $\mathrm{C}_{9} \mathrm{H}_{1} 8 \mathrm{Cl}_{3} \mathrm{O}_{4} \mathrm{P}$ & $13674-84-5$ & 2-Propanol, 1-chloro-, phosphate (3:1) & 4 & 2 \\
\hline 1148,82 & $\mathrm{C}_{8} \mathrm{H}_{5} \mathrm{NO}_{2}$ & $91-56-5$ & $1 \mathrm{H}$-Indole-2,3-dione & 1 & 3 \\
\hline 1093,29 & $\mathrm{C}_{1} 9 \mathrm{H}_{4} 0 \mathrm{O}_{2} \mathrm{Si}$ & $55520-89-3$ & Hexadecanoic acid, trimethylsilyl ester & 7 & 2 \\
\hline 957,14 & $\mathrm{C}_{1} \mathrm{OH}_{22} \mathrm{O}_{3}$ & $29911-28-2$ & 2-Propanol, 1-(2-butoxy-1-methylethoxy)- & 5 & 3 \\
\hline 878,50 & $\mathrm{C}_{8} \mathrm{H}_{10 O}$ & $123-07-9$ & Phenol, 4-ethyl- & 1 & 2 \\
\hline 782,64 & $\mathrm{C}_{14} \mathrm{H}_{11} \mathrm{~N}$ & $611-64-3$ & Acridine, 9-methyl- & 5 & 2 \\
\hline 744,70 & $\mathrm{C}_{12} \mathrm{H}_{22} \mathrm{O}_{2}$ & $1900-69-2$ & Cyclohexanol, 4-(1,1-dimethylethyl)-, acetate, trans- & 2 & 2 \\
\hline 695,40 & $\mathrm{C}_{17} \mathrm{H}_{25} \mathrm{NO}_{4}$ & - & Succinic acid, 2-(dimethylamino)ethyl 4-isopropylphenyl ester & 1 & 2 \\
\hline 677,46 & $\mathrm{C}_{2} 2 \mathrm{H}_{3} 6 \mathrm{O}_{2}$ & - & Benzoic acid, pentadecyl ester & 6 & 3 \\
\hline 632,46 & $\mathrm{C}_{1} 8 \mathrm{H}_{2} 6 \mathrm{O}_{3}$ & $83834-59-7$ & 2-Ethylhexyl trans-4-methoxycinnamate & 1 & 2 \\
\hline 621,12 & $\mathrm{C}_{1} 8 \mathrm{H}_{3} 8 \mathrm{O}_{2} \mathrm{Si}$ & $74367-22-9$ & n-Pentadecanoic acid, trimethylsilyl ester & 4 & 2 \\
\hline 502,22 & $\mathrm{C}_{1} 8 \mathrm{H}_{30}$ & $2719-62-2$ & Benzene, (1-pentylheptyl)- & 1 & 2 \\
\hline 439,92 & $\mathrm{C}_{15} \mathrm{H}_{24}$ & $242794-76-9$ & Bicyclo[5.2.0]nonane, 2-methylene-4,8,8-trimethyl-4-vinyl- & 1 & 2 \\
\hline 435,63 & $\mathrm{C}_{8} \mathrm{H}_{9} \mathrm{NO}_{2}$ & $134-20-3$ & Methyl anthranilate & 1 & 2 \\
\hline 400,28 & $\mathrm{C}_{13} \mathrm{H}_{10} \mathrm{O}$ & $119-61-9$ & Benzophenone & 7 & 2 \\
\hline 356,47 & $\mathrm{C}_{8} \mathrm{H}_{7} \mathrm{NO}$ & $2380-94-1$ & $1 \mathrm{H}-$ Indol-4-ol & 2 & 3 \\
\hline 341,85 & $\mathrm{C} 8 \mathrm{H}_{6} \mathrm{O}_{2}$ & $1074-12-0$ & Phenylglyoxal & 6 & 2 \\
\hline 335,67 & $\mathrm{C}_{7} \mathrm{H}_{9} \mathrm{NO}_{2}$ & $20189-42-8$ & 1H-Pyrrole-2,5-dione, 3-ethyl-4-methyl- & 7 & 3 \\
\hline 299,18 & $\mathrm{C}_{13} \mathrm{H}_{2} 8 \mathrm{O}_{4}$ & $55934-93-5$ & Propanol, [(butoxymethylethoxy)methylethoxy]- & 1 & 2 \\
\hline 253,14 & $\mathrm{C}_{17} \mathrm{H}_{28}$ & $4537-15-9$ & Benzene, (1-butylheptyl)- & 1 & 2 \\
\hline 217,46 & $\mathrm{C}_{9} \mathrm{H}_{7} \mathrm{NO}$ & $487-89-8$ & 1H-Indole-3-carboxaldehyde & 1 & 2 \\
\hline 189,50 & $\mathrm{C}_{7} \mathrm{H} 8 \mathrm{O}$ & $108-39-4$ & Phenol, 3-methyl- & 3 & 2 \\
\hline 186,90 & $\mathrm{C}_{14} \mathrm{H}_{14} \mathrm{O}$ & $19578-70-2$ & Benzene, 1-methyl-2-(phenylmethoxy)- & 5 & 2 \\
\hline 168,37 & $\mathrm{C}_{13} \mathrm{H}_{2} \mathrm{OO}$ & $14901-07-6$ & 3-Buten-2-one, 4-(2,6,6-trimethyl-1-cyclohexen-1-yl)- & 1 & 2 \\
\hline 148,74 & $\mathrm{C}_{14} \mathrm{H}_{8} \mathrm{~N}_{2} \mathrm{~S}_{2}$ & $4271-09-4$ & 2,2'-Bibenzothiazole & 1 & 3 \\
\hline 144,21 & $\mathrm{C}_{10 \mathrm{H}_{14} \mathrm{O}}$ & $99-49-0$ & Carvone & 5 & 2 \\
\hline 124,51 & $\mathrm{C}_{11} \mathrm{H}_{13} \mathrm{ClO}$ & - & 3-Buten-1-ol, 4-chloro-2-methyl-1-phenyl- & 3 & 2 \\
\hline 94,78 & $\mathrm{C}_{1} \mathrm{oH}_{15} \mathrm{NO}_{2} \mathrm{~S}$ & $3622-84-2$ & Benzenesulfonamide, N-butyl- & 3 & 2 \\
\hline 85,86 & $\mathrm{C}_{15} \mathrm{H}_{22} \mathrm{O}_{3}$ & - & Salicylic acid, butyl ether, butyl ester & 1 & 2 \\
\hline 79,19 & $\mathrm{C}_{7} \mathrm{H}_{5} \mathrm{NOS}$ & $934-34-9$ & $2(3 \mathrm{H})$-Benzothiazolone & 2 & 3 \\
\hline 67,22 & $\mathrm{C}_{9} \mathrm{H}_{6} \mathrm{O}_{2}$ & $91-64-5$ & Coumarin & 3 & 2 \\
\hline 46,62 & $\mathrm{C}_{8} \mathrm{H}_{14} \mathrm{O}_{4}$ & $627-93-0$ & Hexanedioic acid, dimethyl ester & 5 & 3 \\
\hline 41,63 & $\mathrm{C} 8 \mathrm{H}_{8} \mathrm{O}_{2}$ & $582-24-1$ & Ethanone, 2-hydroxy-1-phenyl- & 7 & 2 \\
\hline \multirow[t]{11}{*}{12,54} & $\mathrm{C}_{17} \mathrm{H}_{34} \mathrm{O}$ & 55019-46-0 & Hexadecanal, 2-methyl- & 1 & 2 \\
\hline & $\mathrm{C}_{2} \mathrm{OH}_{25} \mathrm{ClN}_{2} \mathrm{O}_{5}$ & & Amlodipine & 6 & 2 \\
\hline & $\mathrm{C}_{3} 8 \mathrm{H}_{52} \mathrm{~N}_{6} \mathrm{O}_{7}$ & & Atazanavir & 5 & 2 \\
\hline & $\mathrm{C}_{14} \mathrm{H}_{22} \mathrm{~N}_{2} \mathrm{O}_{3}$ & & Atenolol & 7 & 2 \\
\hline & $\mathrm{C}_{8} \mathrm{H}_{10} \mathrm{~N}_{4} \mathrm{O}_{2}$ & & Caffeine & 7 & 2 \\
\hline & $\mathrm{C}_{15} \mathrm{H}_{12} \mathrm{~N}_{2} \mathrm{O}$ & & Carbamazepine & 7 & 2 \\
\hline & $\mathrm{C}_{15} \mathrm{H}_{14} \mathrm{~N}_{2} \mathrm{O}_{3}$ & & Carbamazepine, 10, 11-dihydroxy & 7 & 2 \\
\hline & $\mathrm{C}_{15} \mathrm{H}_{14} \mathrm{~N}_{2} \mathrm{O}_{2}$ & & Carbamazepine, 10-hydroxy & 7 & 2 \\
\hline & $\mathrm{C}_{21} \mathrm{H}_{25} \mathrm{ClN}_{2} \mathrm{O}_{3}$ & & Cetirizine & 7 & 2 \\
\hline & $\mathrm{C}_{17} \mathrm{H}_{1} 8 \mathrm{FN}_{3} \mathrm{O}_{3}$ & & Ciprofloxacin & 7 & 2 \\
\hline & $\mathrm{C}_{19} \mathrm{H}_{19} \mathrm{FN}_{2} \mathrm{O}$ & & Citalopram, Desmethyl & 4 & 2 \\
\hline
\end{tabular}


Continued

\begin{tabular}{|c|c|c|c|c|c|}
\hline $\begin{array}{l}\text { Quant } \\
\text { (ng/L) }\end{array}$ & $\begin{array}{l}\text { Molecular } \\
\text { Formula }\end{array}$ & CAS & Common name & $\begin{array}{l}\text { Occur/ } \\
\text { matrix }\end{array}$ & $\begin{array}{r}\text { ID } \\
\text { Level }\end{array}$ \\
\hline & \multicolumn{2}{|l|}{$\mathrm{C}_{2} \mathrm{OH}_{21} \mathrm{FN} 2 \mathrm{O}$} & Citalopram/Escitalopram & 7 & 2 \\
\hline & \multicolumn{2}{|l|}{$\mathrm{C}_{18} \mathrm{H}_{19} \mathrm{ClN}_{4}$} & Clozapine & 7 & 2 \\
\hline & \multicolumn{2}{|l|}{$\mathrm{C}_{1} \mathrm{oH}_{12} \mathrm{~N}_{2} \mathrm{O}$} & Cotinine & 7 & 2 \\
\hline & \multicolumn{2}{|l|}{$\mathrm{C}_{3} \mathrm{H}_{39} \mathrm{NO}_{4}$} & Fexofenadine & 7 & 2 \\
\hline & \multicolumn{2}{|l|}{$\mathrm{C}_{2} 6 \mathrm{H}_{2} 8 \mathrm{Cl}_{2} \mathrm{~N}_{4} \mathrm{O}_{4}$} & Ketoconazole & 7 & 2 \\
\hline & \multicolumn{2}{|l|}{$\mathrm{C}_{9} \mathrm{H}_{7} \mathrm{Cl}_{2} \mathrm{~N}_{5}$} & Lamotrigine & 7 & 2 \\
\hline & \multicolumn{2}{|l|}{$\mathrm{C}_{22} \mathrm{H}_{23} \mathrm{CIN} 6 \mathrm{O}$} & Losartan & 7 & 2 \\
\hline & \multicolumn{2}{|l|}{$\mathrm{C}_{15} \mathrm{H}_{25} \mathrm{NO}_{3}$} & Metoprolol & 7 & 2 \\
\hline & \multicolumn{2}{|l|}{$\mathrm{C}_{17} \mathrm{H}_{19} \mathrm{~N}_{3}$} & Mirtazapine & 7 & 2 \\
\hline & \multicolumn{2}{|l|}{$\mathrm{C}_{10 \mathrm{H}_{1} \mathrm{~N}_{2}}$} & Nicotine & 7 & 2 \\
\hline & \multicolumn{2}{|l|}{$\mathrm{C}_{1} 6 \mathrm{H}_{21} \mathrm{NO}_{2}$} & Propranolol & 7 & 2 \\
\hline & \multicolumn{2}{|l|}{$\mathrm{C}_{21} \mathrm{H}_{25} \mathrm{~N}_{3} \mathrm{O}_{2} \mathrm{~S}$} & Quetiapine & 7 & 2 \\
\hline & \multicolumn{2}{|l|}{$\mathrm{C}_{2} \mathrm{OH}_{2} \mathrm{~N}_{2} \mathrm{O}_{2}$} & Quinine & 7 & 2 \\
\hline & \multicolumn{2}{|l|}{$\mathrm{C}_{11} \mathrm{H}_{11} \mathrm{~N}_{3} \mathrm{O}_{2} \mathrm{~S}$} & Sulfapyridine & 7 & 2 \\
\hline & \multicolumn{2}{|l|}{$\mathrm{C}_{14} \mathrm{H}_{21} \mathrm{~N}_{3} \mathrm{O}_{2} \mathrm{~S}$} & Sumatriptan & 7 & 2 \\
\hline & \multicolumn{2}{|l|}{$\mathrm{C}_{1} 6 \mathrm{H}_{2} 5 \mathrm{NO}_{2}$} & Tramadol & 7 & 2 \\
\hline & \multicolumn{2}{|l|}{$\mathrm{C}_{14} \mathrm{H}_{1} 8 \mathrm{~N}_{4} \mathrm{O}_{3}$} & Trimethoprim & 7 & 2 \\
\hline & \multicolumn{2}{|l|}{$\mathrm{C}_{17} \mathrm{H}_{27} \mathrm{NO}_{2}$} & Venlafaxine & 7 & 2 \\
\hline & \multicolumn{2}{|l|}{$\mathrm{C}_{1} 6 \mathrm{H}_{25} \mathrm{NO}_{2}$} & Venlafaxine,-O-demethyl- & 8 & 2 \\
\hline & $\mathrm{C}_{9} \mathrm{H}_{11} \mathrm{NO}_{2}$ & $94-09-7$ & BENZOCAINE & 10 & 2 \\
\hline & $\mathrm{C}_{8} \mathrm{H}_{9} \mathrm{NO}_{2}$ & $103-90-2$ & paracetamol & 11 & 2 \\
\hline & $\mathrm{C}_{7} \mathrm{H}_{7} \mathrm{NO}_{5} \mathrm{~S}$ & $121-03-9$ & 4-nitrotoluene-2-sulphonic acid & 2 & 2 \\
\hline & $\mathrm{C}_{12} \mathrm{H}_{24} \mathrm{O}_{2}$ & $143-07-7$ & Neo-Fat $12-43$ & 1 & 2 \\
\hline & $\mathrm{C}_{1} 8 \mathrm{H}_{3} 6 \mathrm{O}_{3}$ & $106-14-9$ & 12-hydroxystearic acid & 1 & 2 \\
\hline & & $1799-84-4$ & 4:2 FTMAC & 5 & 2 \\
\hline & & $106-44-5$ & p-cresol & 1 & 2 \\
\hline & & $123-08-0$ & 4-hydroxybenzaldehyde & 7 & 2 \\
\hline & & $1074-86-8$ & 4-formyl indole & 6 & 2 \\
\hline & & $3233-32-7$ & 4-acetoxyphenol & 1 & 2 \\
\hline & & $103-90-2$ & paracetamol & 5 & 2 \\
\hline
\end{tabular}


Table 17: Effluent Faroe Islands

\begin{tabular}{|c|c|c|c|c|c|}
\hline $\begin{array}{l}\text { Quant } \\
\text { (ng/L) }\end{array}$ & $\begin{array}{l}\text { Molecular } \\
\text { Formula }\end{array}$ & CAS & Common name & $\begin{array}{l}\text { Occur/ } \\
\text { matrix }\end{array}$ & $\begin{array}{r}\text { ID } \\
\text { Level }\end{array}$ \\
\hline 6515,32 & $\mathrm{C}_{8} \mathrm{H}_{10 O}$ & $122-99-6$ & Ethanol, 2-phenoxy- & 6 & 2 \\
\hline 2910,65 & $\mathrm{C}_{1} \mathrm{OH} 2 \mathrm{OO}$ & $89-78-1$ & DL-Menthol & 3 & 2 \\
\hline 2366,64 & $\mathrm{C}_{8} \mathrm{H}_{10} \mathrm{~N}_{4} \mathrm{O}_{2}$ & $58-08-2$ & Caffeine & 7 & 3 \\
\hline 1909,34 & $\mathrm{C}_{10} \mathrm{H}_{16}$ & $3760-14-3$ & 1,5-Cyclooctadiene, 1,5-dimethyl- & 7 & 3 \\
\hline 1782,73 & $\mathrm{C}_{9} \mathrm{H}_{12} \mathrm{O}$ & $617-94-7$ & Benzenemethanol, à,à-dimethyl- & 1 & 2 \\
\hline 1472,49 & $\mathrm{Cg} H g \mathrm{~N}$ & $83-34-1$ & 1H-Indole, 3-methyl- & 2 & 2 \\
\hline 1179,06 & $\mathrm{C}_{10 \mathrm{H}_{22} \mathrm{O}}$ & $78-69-3$ & 3-Octanol, 3,7-dimethyl- & 3 & 2 \\
\hline 1019,71 & $\mathrm{C}_{14} \mathrm{H}_{31} \mathrm{~N}$ & $112-18-5$ & 1-Dodecanamine, N,N-dimethyl- & 1 & 3 \\
\hline 343,58 & $\mathrm{C}_{19} \mathrm{H}_{4} \mathrm{OO} 2 \mathrm{Si}$ & $55520-89-3$ & Hexadecanoic acid, trimethylsilyl ester & 7 & 2 \\
\hline 263,73 & $\mathrm{C}_{10} \mathrm{H}_{14} \mathrm{O}$ & $99-49-0$ & Carvone & 5 & 2 \\
\hline 258,63 & $\mathrm{C} 8 \mathrm{H}_{6} \mathrm{O}_{2}$ & $1074-12-0$ & Phenylglyoxal & 6 & 2 \\
\hline 231,30 & $\mathrm{C}_{1} 8 \mathrm{H}_{2} 6 \mathrm{O}$ & $1222-05-5$ & Galaxolide & 6 & 2 \\
\hline 194,73 & $\mathrm{C}_{10 \mathrm{H}_{22} \mathrm{O}_{3}}$ & $29911-28-2$ & 2-Propanol, 1-(2-butoxy-1-methylethoxy)- & 5 & 3 \\
\hline 183,48 & $\mathrm{C}_{7} \mathrm{H}_{9} \mathrm{NO}_{2}$ & $20189-42-8$ & 1H-Pyrrole-2,5-dione, 3-ethyl-4-methyl- & 7 & 3 \\
\hline 161,29 & $\mathrm{C}_{12} \mathrm{H}_{24} \mathrm{O}_{3}$ & $74367-34-3$ & Propanoic acid, 2-methyl-, 3-hydroxy-2,4,4-trimethylpentyl ester & 7 & 2 \\
\hline 104,03 & $\mathrm{C} 8 \mathrm{H}_{8} \mathrm{O}_{2}$ & $582-24-1$ & Ethanone, 2-hydroxy-1-phenyl- & 7 & 2 \\
\hline 78,39 & $\mathrm{C}_{12} \mathrm{H}_{22} \mathrm{O}_{2}$ & $1900-69-2$ & Cyclohexanol, 4-(1,1-dimethylethyl)-, acetate, trans- & 2 & 2 \\
\hline 70,46 & $\mathrm{C}_{13} \mathrm{H}_{10} \mathrm{O}$ & $119-61-9$ & Benzophenone & 7 & 2 \\
\hline 60,12 & $\mathrm{C}_{1} 8 \mathrm{H}_{2} 8 \mathrm{O}_{2}$ & $6316-30-9$ & Benzoic acid, undecyl ester & 7 & 3 \\
\hline 56,99 & $\mathrm{C}_{2} \mathrm{OH}_{32} \mathrm{O}_{2}$ & - & Benzoic acid, tridecyl ester & 7 & 3 \\
\hline 42,46 & $\mathrm{C}_{8} \mathrm{H}_{7} \mathrm{NS}_{2}$ & $615-22-5$ & Benzothiazole, 2-(methylthio)- & 5 & 2 \\
\hline 37,64 & $\mathrm{C}_{17} \mathrm{H}_{3} 6 \mathrm{O}_{2} \mathrm{Si}$ & $18603-17-3$ & Tetradecanoic acid, trimethylsilyl ester & 5 & 2 \\
\hline 34,13 & $\mathrm{C}_{2} 2 \mathrm{H}_{3} 6 \mathrm{O}_{2}$ & - & Benzoic acid, pentadecyl ester & 6 & 3 \\
\hline \multirow[t]{39}{*}{23,35} & $\mathrm{C}_{9} \mathrm{H}_{6} \mathrm{O}_{2}$ & $91-64-5$ & Coumarin & 3 & 2 \\
\hline & $\mathrm{C}_{2} \mathrm{OH}_{23} \mathrm{~N}$ & & Amitriptyline & 6 & 2 \\
\hline & $\mathrm{C}_{2} \mathrm{OH}_{25} \mathrm{ClN}_{2} \mathrm{O}_{5}$ & & Amlodipine & 6 & 2 \\
\hline & $\mathrm{C}_{14} \mathrm{H}_{22} \mathrm{~N}_{2} \mathrm{O}_{3}$ & & Atenolol & 7 & 2 \\
\hline & $\mathrm{C}_{3} 8 \mathrm{H}_{72} \mathrm{~N}_{2} \mathrm{O}_{12}$ & & Azithromycin & 6 & 2 \\
\hline & $\mathrm{C}_{8} \mathrm{H}_{10} \mathrm{~N}_{4} \mathrm{O}_{2}$ & & Caffeine & 7 & 2 \\
\hline & $\mathrm{C}_{15} \mathrm{H}_{12} \mathrm{~N}_{2} \mathrm{O}$ & & Carbamazepine & 7 & 2 \\
\hline & $\mathrm{C}_{15} \mathrm{H}_{14} \mathrm{~N}_{2} \mathrm{O}_{3}$ & & Carbamazepine, 10, 11-dihydroxy & 7 & 2 \\
\hline & $\mathrm{C}_{15} \mathrm{H}_{14} \mathrm{~N}_{2} \mathrm{O}_{2}$ & & Carbamazepine, 10 -hydroxy & 7 & 2 \\
\hline & $\mathrm{C}_{21} \mathrm{H}_{25} \mathrm{ClN}_{2} \mathrm{O}_{3}$ & & Cetirizine & 7 & 2 \\
\hline & $\mathrm{C}_{17} \mathrm{H}_{1} 8 \mathrm{FN}_{3} \mathrm{O}_{3}$ & & Ciprofloxacin & 7 & 2 \\
\hline & $\mathrm{C}_{2} \mathrm{OH}_{21} \mathrm{FN}_{2} \mathrm{O}$ & & Citalopram/Escitalopram & 7 & 2 \\
\hline & $\mathrm{C}_{3} 8 \mathrm{H}_{69} \mathrm{NO}_{13}$ & & Clarithromycin & 6 & 2 \\
\hline & $\mathrm{C}_{18} \mathrm{H}_{19} \mathrm{ClN}_{4}$ & & Clozapine & 7 & 2 \\
\hline & $\mathrm{C}_{17} \mathrm{H}_{17} \mathrm{ClN}_{4}$ & & Clozapine, Desmethyl & 6 & 2 \\
\hline & $\mathrm{C}_{1} 8 \mathrm{H}_{21} \mathrm{NO}_{3}$ & & Codeine & 6 & 2 \\
\hline & $\mathrm{C}_{1} \mathrm{OH}_{12} \mathrm{~N}_{2} \mathrm{O}$ & & Cotinine & 7 & 2 \\
\hline & $\mathrm{C}_{22} \mathrm{H}_{2} 6 \mathrm{~N}_{2} \mathrm{O}_{4} \mathrm{~S}$ & & Diltiazem & 6 & 2 \\
\hline & $\mathrm{C}_{2} 4 \mathrm{H}_{4} \mathrm{ON}_{8} \mathrm{O}_{4}$ & & Dipyridamol & 4 & 2 \\
\hline & $\mathrm{C}_{32} \mathrm{H}_{39} \mathrm{NO}_{4}$ & & Fexofenadine & 7 & 2 \\
\hline & $\mathrm{C}_{1} 8 \mathrm{H}_{21} \mathrm{NO}_{3}$ & & Hydrocodone & 6 & 2 \\
\hline & $\mathrm{C}_{2} 6 \mathrm{H}_{2} 8 \mathrm{Cl}_{2} \mathrm{~N}_{4} \mathrm{O}_{4}$ & & Ketoconazole & 7 & 2 \\
\hline & $\mathrm{C}_{9} \mathrm{H}_{7} \mathrm{Cl}_{2} \mathrm{~N}_{5}$ & & Lamotrigine & 7 & 2 \\
\hline & $\mathrm{C}_{22} \mathrm{H}_{23} \mathrm{ClN} 6 \mathrm{O}$ & & Losartan & 7 & 2 \\
\hline & $\mathrm{C}_{2} \mathrm{OH}_{23} \mathrm{~N}$ & & Maprotiline & 6 & 2 \\
\hline & $\mathrm{C}_{14} \mathrm{H}_{22} \mathrm{ClN}_{3} \mathrm{O}_{2}$ & & Metoclopramide & 6 & 2 \\
\hline & $\mathrm{C}_{15} \mathrm{H}_{25} \mathrm{NO}_{3}$ & & Metoprolol & 7 & 2 \\
\hline & $\mathrm{C}_{17} \mathrm{H}_{19} \mathrm{~N}_{3}$ & & Mirtazapine & 7 & 2 \\
\hline & 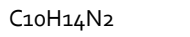 & & Nicotine & 7 & 2 \\
\hline & $\mathrm{C}_{15} \mathrm{H}_{11} \mathrm{ClN}_{2} \mathrm{O}_{2}$ & & Oxazepam & 6 & 2 \\
\hline & $\mathrm{C}_{1} 6 \mathrm{H}_{21} \mathrm{NO}_{2}$ & & Propranolol & 7 & 2 \\
\hline & $\mathrm{C}_{21} \mathrm{H}_{25} \mathrm{~N}_{3} \mathrm{O}_{2} \mathrm{~S}$ & & Quetiapine & 7 & 2 \\
\hline & $\mathrm{C}_{2} \mathrm{OH}_{2} \mathrm{~N}_{2} \mathrm{O}_{2}$ & & Quinine & 7 & 2 \\
\hline & $\mathrm{C}_{12} \mathrm{H}_{2} \mathrm{ON}_{2} \mathrm{O}_{3} \mathrm{~S}$ & & Sotalol & 6 & 2 \\
\hline & $\mathrm{C}_{11} \mathrm{H}_{11} \mathrm{~N}_{3} \mathrm{O}_{2} \mathrm{~S}$ & & Sulfapyridine & 7 & 2 \\
\hline & $\mathrm{C}_{14} \mathrm{H}_{21} \mathrm{~N}_{3} \mathrm{O}_{2} \mathrm{~S}$ & & Sumatriptan & 7 & 2 \\
\hline & $\mathrm{C}_{1} 6 \mathrm{H}_{25} \mathrm{NO}_{2}$ & & Tramadol & 7 & 2 \\
\hline & $\mathrm{C}_{14} \mathrm{H}_{1} 8 \mathrm{~N}_{4} \mathrm{O}_{3}$ & & Trimethoprim & 7 & 2 \\
\hline & $\mathrm{C}_{17} \mathrm{H}_{27} \mathrm{NO}_{2}$ & & Venlafaxine & 7 & 2 \\
\hline
\end{tabular}


Continued

\begin{tabular}{|c|c|c|c|c|c|}
\hline $\begin{array}{l}\text { Quant } \\
\text { (ng/L) }\end{array}$ & $\begin{array}{l}\text { Molecular } \\
\text { Formula }\end{array}$ & CAS & Common name & $\begin{array}{l}\text { Occur/ } \\
\text { matrix }\end{array}$ & $\begin{array}{r}\text { ID } \\
\text { Level }\end{array}$ \\
\hline & $\mathrm{C}_{1} 6 \mathrm{H}_{25} \mathrm{NO}_{2}$ & & Venlafaxine,-O-demethyl- & 7 & 2 \\
\hline & $\mathrm{C}_{7} \mathrm{H}_{7} \mathrm{NO}_{5} \mathrm{~S}$ & $121-03-9$ & 4-nitrotolvene-2-sulphonic acid & 2 & 2 \\
\hline & $\mathrm{C}_{9} \mathrm{H}_{11} \mathrm{NO}_{3}$ & $69-96-5$ & beta-hydroxyphenylalanine & 1 & 2 \\
\hline & $\mathrm{C}_{17} \mathrm{H}_{34} \mathrm{O}_{2}$ & $6309-51-9$ & isopentyl laurate & 3 & 2 \\
\hline & $\mathrm{C}_{17} \mathrm{H}_{34} \mathrm{O}_{2}$ & $110-27-0$ & Wickenol 101 & 3 & 2 \\
\hline & $\mathrm{C}_{17} \mathrm{H}_{34} \mathrm{O}_{2}$ & $112-39-0$ & methyl palmitate & 2 & 2 \\
\hline & $\mathrm{C}_{8} \mathrm{H}_{5} \mathrm{NO}_{2}$ & $85-41-6$ & $1 \mathrm{H}$-Isoindole-1,3(2H)-dione & 2 & 2 \\
\hline & $\mathrm{C}_{7} \mathrm{H}_{9} \mathrm{NO}_{2}$ & $30652-11-0$ & Deferiprone & 2 & 2 \\
\hline & $\mathrm{C}_{9} \mathrm{H}_{11} \mathrm{NO}_{2}$ & $938-73-8$ & ethenzamide & 1 & 2 \\
\hline & $\mathrm{C}_{1} 6 \mathrm{H}_{32} \mathrm{O}_{2}$ & $2306-88-9$ & octyl octanoate & 2 & 2 \\
\hline & $\mathrm{C}_{1} 6 \mathrm{H}_{32} \mathrm{O}_{2}$ & $57-10-3$ & Hexadecanoic acid & 2 & 2 \\
\hline & $\mathrm{C}_{8} \mathrm{H}_{9} \mathrm{NO}_{2}$ & $103-90-2$ & paracetamol & 5 & 2 \\
\hline & $\mathrm{C}_{9} \mathrm{H}_{19} \mathrm{NO}_{4}$ & $16485-10-2$ & panthenol, DL-form & 2 & 2 \\
\hline & $\mathrm{C}_{11} \mathrm{H}_{12} \mathrm{~N}_{2} \mathrm{O}_{2}$ & $73-22-3$ & Tryptophan & 1 & 2 \\
\hline & & $1799-84-4$ & 4:2 FTMAC & 5 & 2 \\
\hline & & $80-09-1$ & bisphenol S & 3 & 1 \\
\hline & & $13595-25-0$ & bisphenol M & 5 & 1 \\
\hline & & $123-08-0$ & 4-hydroxybenzaldehyde & 7 & 2 \\
\hline & & $1074-86-8$ & 4-formyl indole & 6 & 2 \\
\hline & & $27503-81-7$ & PBS (Phenylbenzimidazole sulfonic acid) & 7 & 2 \\
\hline & & $103-90-2$ & paracetamol & 5 & 2 \\
\hline
\end{tabular}


Table 18: Sediment Finland

\begin{tabular}{|c|c|c|c|c|c|}
\hline $\begin{array}{l}\text { Conc } \\
\text { (ng/g) }\end{array}$ & $\begin{array}{l}\text { Molecular } \\
\text { Formula }\end{array}$ & CAS & Common name & $\begin{array}{l}\text { Occur/ } \\
\text { matrix }\end{array}$ & $\begin{array}{r}\text { ID } \\
\text { Level }\end{array}$ \\
\hline 272,77 & $\mathrm{C}_{23} \mathrm{H}_{3} 6 \mathrm{O}_{4}$ & - & Phthalic acid, hept-3-yl octyl ester & 6 & 2 \\
\hline 143,64 & $\mathrm{C}_{1} 6 \mathrm{H}_{10}$ & $206-44-0$ & Fluoranthene & 6 & 2 \\
\hline 136,61 & $\mathrm{C}_{1} 6 \mathrm{H}_{10}$ & $129-00-0$ & Pyrene & 6 & 2 \\
\hline 99,31 & $\mathrm{C}_{2} \mathrm{OH}_{12}$ & $205-99-2$ & Benz[b]fluoranthene & 1 & 2 \\
\hline 82,86 & $\mathrm{C}_{14} \mathrm{H}_{10}$ & $85-01-8$ & Phenanthrene & 6 & 2 \\
\hline 63,91 & $\mathrm{C}_{1} 8 \mathrm{H}_{12}$ & $217-59-4$ & Triphenylene & 6 & 2 \\
\hline 58,31 & $\mathrm{C}_{3} \mathrm{OH}_{4} 8 \mathrm{O}$ & - & $\begin{array}{l}\text { 4,4,6a,6b,8a,11,11,14b-Octamethyl-1,4,4a,5,6,6a,6b,7,8,8a,9,10,11, } \\
12,12 \mathrm{a}, 14,14 \mathrm{a}, 14 \mathrm{~b} \text {-octadecahydro-2H-picen-3-one }\end{array}$ & 2 & 3 \\
\hline 45,63 & $\mathrm{C}_{2} \mathrm{OH}_{12}$ & $198-55-0$ & Perylene & 5 & 2 \\
\hline 44,49 & $\mathrm{C}_{3} \mathrm{OH}_{4} 8 \mathrm{O}$ & $1617-70-5$ & Lupenone & 2 & 3 \\
\hline 43,28 & $\mathrm{C}_{1} 8 \mathrm{H}_{12}$ & $56-55-3$ & Benz[a]anthracene & 6 & 2 \\
\hline 41,80 & $\mathrm{C}_{13} \mathrm{H}_{10} \mathrm{O}$ & $119-61-9$ & Benzophenone & 7 & 2 \\
\hline 40,34 & $\mathrm{C}_{7} \mathrm{H}_{5} \mathrm{NS}$ & $95-16-9$ & Benzothiazole & 6 & 2 \\
\hline 40,23 & $\mathrm{C}_{2} \mathrm{OH}_{12}$ & $192-97-2$ & Benzo[e]pyrene & 5 & 2 \\
\hline 38,80 & $\mathrm{C}_{14} \mathrm{H}_{14} \mathrm{O}$ & $19578-70-2$ & Benzene, 1-methyl-2-(phenylmethoxy)- & 5 & 2 \\
\hline 36,83 & $\mathrm{C}_{9} \mathrm{H}_{12} \mathrm{O}_{2}$ & $1125-21-9$ & 2,6,6-Trimethyl-2-cyclohexene-1,4-dione & 6 & 2 \\
\hline 36,06 & $\mathrm{C}_{13} \mathrm{H}_{1} 6 \mathrm{O}$ & $55591-10-1$ & Ethanone, 1-(2,3-dihydro-1,1-dimethyl-1H-inden-4-yl)- & 4 & 3 \\
\hline 34,55 & $\mathrm{C}_{22} \mathrm{H}_{12}$ & $193-39-5$ & Indeno[1,2,3-cd]pyrene & 4 & 2 \\
\hline 33,75 & $\mathrm{C}_{3} \mathrm{OH}_{50}$ & $1615-91-4$ & Diploptene & 4 & 3 \\
\hline 30,73 & $\mathrm{C}_{2} \mathrm{OH}_{12}$ & $50-32-8$ & Benzo[a]pyrene & 5 & 2 \\
\hline 28,93 & $\mathrm{C}_{11} \mathrm{H}_{16} 6 \mathrm{O}_{2}$ & $15356-74-8$ & $2(4 \mathrm{H})$-Benzofuranone, 5,6,7,7a-tetrahydro-4,4,7a-trimethyl- & 5 & 2 \\
\hline 24,53 & $\mathrm{C}_{1} \mathrm{OH} 8$ & $91-20-3$ & Naphthalene & 4 & 2 \\
\hline 21,25 & $\mathrm{C}_{18} \mathrm{H}_{18} 8$ & $66552-97-4$ & 2-Isopropyl-10-methylphenanthrene & 5 & 3 \\
\hline 21,01 & $\mathrm{C}_{9} \mathrm{H}_{18} 8 \mathrm{Cl}_{3} \mathrm{O}_{4} \mathrm{P}$ & $13674-84-5$ & 2-Propanol, 1-chloro-, phosphate (3:1) & 6 & 2 \\
\hline 20,26 & $\mathrm{C}_{17} \mathrm{H}_{12}$ & $199-94-0$ & $7 \mathrm{H}$-Benzo[de]anthracene & 4 & 3 \\
\hline 19,27 & $\mathrm{C}_{7} \mathrm{H}_{9} \mathrm{NO}_{2}$ & $20189-42-8$ & 1H-Pyrrole-2,5-dione, 3-ethyl-4-methyl- & 7 & 3 \\
\hline 19,11 & $\mathrm{C}_{12} \mathrm{H}_{6} \mathrm{Cl}_{4}$ & $35693-99-3$ & 1,1'-Biphenyl, 2,2',5,5'-tetramethyl- & 6 & 3 \\
\hline 17,04 & $\mathrm{C}_{1} 6 \mathrm{H}_{1} 6$ & $7694-30-6$ & Benzene, $1,1^{\prime}-(1,2$-cyclobutanediyl)bis-, cis- & 5 & 2 \\
\hline 16,61 & $\mathrm{C}_{19} \mathrm{H}_{14}$ & $82979-72-4$ & 8,9-Dihydro-7H-cyclopenta[a]pyrene & 5 & 3 \\
\hline 16,36 & $\mathrm{C}_{14} \mathrm{H}_{10}$ & $120-12-7$ & Anthracene & 6 & 2 \\
\hline 14,98 & $\mathrm{C}_{15} \mathrm{H}_{12}$ & $27410-55-5$ & 8,9-Dihydrocyclopenta[def]phenanthrene & 5 & 3 \\
\hline 13,31 & $\mathrm{C}_{11} \mathrm{H}_{14} \mathrm{O}_{4}$ & - & 1-Methyl-1,3-cyclohexadiene-5,6-diol diacetate & 3 & 3 \\
\hline 12,83 & $\mathrm{C}_{1} 6 \mathrm{H}_{10} \mathrm{O}$ & $205-39-0$ & Benzo(b)naphtho(1,2-d)furan & 5 & 3 \\
\hline 12,71 & $\mathrm{C}_{17} \mathrm{H}_{12}$ & - & $7 \mathrm{H}$-Benzo[c]fluorene (isomer) & 13 & 3 \\
\hline 12,53 & $\mathrm{C}_{7} \mathrm{H}_{7} \mathrm{NO}_{2}$ & $21494-57-5$ & $1 \mathrm{H}$-Pyrrole-2,5-dione, 3-ethenyl-4-methyl- & 6 & 3 \\
\hline 12,42 & $\mathrm{C}_{1} \mathrm{OH}_{16}$ & $3760-14-3$ & 1,5-Cyclooctadiene, 1,5-dimethyl- & 4 & 3 \\
\hline 11,17 & $\mathrm{C}_{5} \mathrm{H}_{9} \mathrm{NO}_{2}$ & $4394-85-8$ & N-Formylmorpholine & 2 & 2 \\
\hline 11,15 & $\mathrm{C}_{22} \mathrm{H}_{12}$ & $193-43-1$ & Indeno[1,2,3-cd]fluoranthene & 4 & 2 \\
\hline 11,10 & $\mathrm{C}_{22} \mathrm{H}_{34} \mathrm{O}_{4}$ & - & Phthalic acid, nonyl 2-pentyl ester & 2 & 2 \\
\hline 10,70 & $\mathrm{C}_{12} \mathrm{H} 8 \mathrm{O}$ & $132-64-9$ & Dibenzofuran & 5 & 2 \\
\hline 9,94 & $\mathrm{C}_{15} \mathrm{H}_{10}$ & $83469-43-6$ & $6 \mathrm{H}-\mathrm{Cyclobuta}[\mathrm{jk}]$ phenanthrene & 5 & 2 \\
\hline 9,89 & $\mathrm{C}_{1} 8 \mathrm{H}_{30}$ & $2719-62-2$ & Benzene, (1-pentylheptyl)- & 4 & 2 \\
\hline 9,87 & $\mathrm{C}_{1} 6 \mathrm{H}_{10 S}$ & $205-43-6$ & Benzo[b]naphtho[1,2-d]thiophene & 5 & 2 \\
\hline 9,31 & $\mathrm{C}_{12} \mathrm{H}_{24} \mathrm{O}_{3}$ & $74367-34-3$ & Propanoic acid, 2-methyl-, 3-hydroxy-2,4,4-trimethylpentyl ester & 5 & 2 \\
\hline 9,22 & $\mathrm{C}_{12} \mathrm{H}_{12}$ & - & Naphthalene, dimethyl- & 10 & 3 \\
\hline 8,78 & $\mathrm{C}_{13} \mathrm{H}_{14}$ & $829-26-5$ & Naphthalene, 2,3,6-trimethyl- & 4 & 2 \\
\hline 8,73 & $\mathrm{C}_{13} \mathrm{H}_{10}$ & $86-73-7$ & Fluorene & 4 & 2 \\
\hline 8,64 & $\mathrm{C}_{17} \mathrm{H}_{12}$ & - & $7 \mathrm{H}$-Benzo[c]fluorene (isomer) & 13 & 3 \\
\hline 8,56 & $\mathrm{C}_{1} 8 \mathrm{H}_{22}$ & $6566-19-4$ & 10,18-Bisnorabieta-5,7,9(10),11,13-pentaene & 3 & 3 \\
\hline 8,54 & $\mathrm{C}_{15} \mathrm{H}_{28}$ & $54832-83-6$ & ${ }_{1} \mathrm{H}$-Indene, octahydro-2,2,4,4,7,7-hexamethyl-, trans- & 3 & 3 \\
\hline 8,10 & $\mathrm{C}_{13} \mathrm{H} 8 \mathrm{O}$ & $548-39-0$ & $1 \mathrm{H}$-Phenalen-1-one & 4 & 2 \\
\hline 7,74 & $\mathrm{C}_{8} \mathrm{H}_{7} \mathrm{NS}_{2}$ & $615-22-5$ & Benzothiazole, 2-(methylthio)- & 2 & 2 \\
\hline 7,53 & $\mathrm{C} 8 \mathrm{H}_{8} \mathrm{O}_{3}$ & $121-33-5$ & Vanillin & 2 & 2 \\
\hline 7,47 & $\mathrm{C}_{9} \mathrm{H}_{10} \mathrm{O}_{2}$ & $101-41-7$ & Phenylacetic acid, methyl ester & 2 & 2 \\
\hline 7,38 & $\mathrm{C}_{12} \mathrm{H}_{6} \mathrm{Cl}_{4}$ & - & 1,1'-Biphenyl, 2,2',5,5'-tetramethyl- (isomer) & 3 & 3 \\
\hline 7,34 & $\mathrm{C}_{15} \mathrm{H}_{12}$ & $832-71-3$ & Phenanthrene, 3-methyl- & 4 & 2 \\
\hline 7,16 & $\mathrm{C}_{17} \mathrm{H}_{28}$ & $4537-15-9$ & Benzene, (1-butylheptyl)- & 3 & 2 \\
\hline 6,99 & $\mathrm{C}_{16} \mathrm{H}_{12}$ & $6232-48-0$ & Acephenanthrylene, 4,5-dihydro- & 5 & 2 \\
\hline 6,87 & $\mathrm{C}_{11} \mathrm{H}_{10}$ & $91-57-6$ & Naphthalene, 2-methyl- & 6 & 2 \\
\hline 6,81 & $\mathrm{C}_{17} \mathrm{H}_{12}$ & - & 7H-Benzo[c]fluorene (isomer) & 13 & 3 \\
\hline 5,73 & $\mathrm{C}_{17} \mathrm{H}_{16}$ & $3674-73-5$ & Phenanthrene, 2,3,5-trimethyl- & 4 & 2 \\
\hline 5,56 & $\mathrm{C}_{11} \mathrm{H}_{18} \mathrm{O}_{4}$ & & Fumaric acid, ethyl 3-methylbut-2-yl ester & 3 & 2 \\
\hline 5,49 & $\mathrm{C}_{17} \mathrm{H}_{12}$ & $243-17-4$ & $11 \mathrm{H}$-Benzo[b]fluorene & 5 & 2 \\
\hline
\end{tabular}


Continued

\begin{tabular}{|c|c|c|c|c|}
\hline $\begin{array}{l}\text { Conc } \\
\text { (ng/g) }\end{array}$ & $\begin{array}{l}\text { Molecular } \\
\text { Formula }\end{array}$ & CAS & Common name & $\begin{array}{l}\text { Occur/ } \\
\text { matrix }\end{array}$ \\
\hline
\end{tabular}

\begin{tabular}{|c|c|c|c|c|c|}
\hline 5,02 & $\mathrm{C}_{12} \mathrm{H}_{12}$ & - & Naphthalene, dimethyl- & 10 & 3 \\
\hline 4,98 & $\mathrm{C}_{16} \mathrm{H}_{14}$ & $3674-69-9$ & Phenanthrene, 4,5-dimethyl- & 5 & 2 \\
\hline 4,97 & $\mathrm{C}_{17} \mathrm{H}_{12}$ & - & $7 \mathrm{H}$-Benzo[c]fluorene (isomer) & 13 & 3 \\
\hline 4,94 & $\mathrm{C}_{15} \mathrm{H} 8 \mathrm{O}$ & $5737-13-3$ & Cyclopenta(def)phenanthrenone & 4 & 2 \\
\hline 4,89 & $\mathrm{C}_{1} 6 \mathrm{H}_{11} \mathrm{~N}$ & - & 11H-Benzo[a]carbazole (isomer) & 7 & 3 \\
\hline 4,76 & $\mathrm{C}_{9} \mathrm{H}_{14} \mathrm{O} 6$ & $102-76-1$ & Triacetin & 3 & 2 \\
\hline 4,75 & $\mathrm{C}_{21} \mathrm{H}_{3} 6$ & $641-85-0$ & Allopregnane & 3 & 3 \\
\hline 4,72 & $\mathrm{C}_{1} 8 \mathrm{H}_{10}$ & $203-12-3$ & Benzo[ghi]fluoranthene & 3 & 2 \\
\hline 4,67 & $\mathrm{C}_{1} 6 \mathrm{H}_{100}$ & $200-23-7$ & Benzo[kl]xanthene & 4 & 3 \\
\hline 4,45 & $\mathrm{C}_{2} \mathrm{OH}_{12}$ & $205-82-3$ & Benzo[j]fluoranthene & 4 & 2 \\
\hline 3,92 & $\mathrm{C}_{16} \mathrm{H}_{14}$ & $1576-67-6$ & Phenanthrene, 3,6-dimethyl- & 5 & 2 \\
\hline 3,89 & $\mathrm{C}_{14} \mathrm{H}_{8} \mathrm{O}_{2}$ & $84-65-1$ & 9,10-Anthracenedione & 5 & 2 \\
\hline 3,79 & $\mathrm{C}_{17} \mathrm{H}_{10 O}$ & $82-05-3$ & $7 \mathrm{H}$-Benz[de]anthracen-7-one & 4 & 2 \\
\hline 3,75 & $\mathrm{C}_{11} \mathrm{H}_{10}$ & $90-12-0$ & Naphthalene, 1-methyl- & 6 & 2 \\
\hline 3,70 & $\mathrm{C}_{9} \mathrm{H}_{7} \mathrm{NO}$ & $487-89-8$ & ${ }_{1} \mathrm{H}$-Indole-3-carboxaldehyde & 3 & 2 \\
\hline 3,55 & $\mathrm{C}_{1} 6 \mathrm{H}_{10 O}$ & $243-42-5$ & Benzo[b]naphtho[2,3-d]furan & 4 & 3 \\
\hline 3,31 & $\mathrm{C}_{21} \mathrm{H}_{14}$ & $63104-32-5$ & 10-Methylbenzo(a)pyrene & 4 & 3 \\
\hline 3,26 & $\mathrm{C}_{1} 6 \mathrm{H}_{10 S}$ & $227-86-1$ & Anthra(1,2-b)thiophene & 3 & 3 \\
\hline 3,22 & $\mathrm{C}_{1} 8 \mathrm{H}_{10 O}$ & $191-37-7$ & Naphtho[2,1,8,7-klmn]xanthene & 4 & 3 \\
\hline 3,18 & $\mathrm{C}_{1} 8 \mathrm{H}_{12}$ & $195-19-7$ & Benzo[c]phenanthrene & 5 & 2 \\
\hline 3,15 & $\mathrm{C}_{13} \mathrm{H}_{10} \mathrm{O}$ & $92-83-1$ & $9 \mathrm{H}$-Xanthene & 5 & 2 \\
\hline 2,95 & $\mathrm{C}_{19} \mathrm{H}_{12}$ & $50861-05-7$ & 9H-Cyclopenta[a]pyrene & 5 & 2 \\
\hline 2,91 & $\mathrm{C}_{18} \mathrm{H}_{14} \mathrm{~S}$ & $24964-16-7$ & Benzo[b]naphtho[2,3-d]thiophene, 7,8-dimethyl- & 3 & 3 \\
\hline 2,89 & $\mathrm{C}_{1} \mathrm{OH}_{12} \mathrm{O}$ & $487-68-3$ & Benzaldehyde, 2,4,6-trimethyl- & 3 & 3 \\
\hline 2,80 & $\mathrm{C}_{2} \mathrm{OH}_{16}$ & 35187-19-0 & 4,12-Dimethylbenz[a]anthracene & 4 & 3 \\
\hline 2,80 & $\mathrm{C}_{13} \mathrm{H}_{2} \mathrm{OO}$ & $14901-07-6$ & 3-Buten-2-one, 4-(2,6,6-trimethyl-1-cyclohexen-1-yl)- & 3 & 2 \\
\hline 2,77 & $\mathrm{C}_{11} \mathrm{H}_{20}$ & $2958-76-1$ & Naphthalene, decahydro-2-methyl- & 2 & 2 \\
\hline 2,61 & $\mathrm{C}_{15} \mathrm{H}_{22}$ & $483-77-2$ & Naphthalene, 1,2,3,4-tetrahydro-1,6-dimethyl-4-(1-methylethyl)-, (1S-cis)- & 3 & 2 \\
\hline 2,53 & $\mathrm{C}_{1} 6 \mathrm{H}_{11} \mathrm{~N}$ & $243-28-7$ & Benzo(b)carbazole & 2 & 2 \\
\hline 2,50 & $\mathrm{C}_{21} \mathrm{H}_{14}$ & $239-85-0$ & $13 \mathrm{H}$-Dibenzo[a,h]fluorene & 5 & 3 \\
\hline 2,46 & $\mathrm{C}_{15} \mathrm{H}_{12}$ & $832-64-4$ & Phenanthrene, 4-methyl- & 4 & 2 \\
\hline 2,39 & $\mathrm{C}_{21} \mathrm{H}_{14}$ & $241-28-1$ & $8 \mathrm{H}$-Indeno[2,1-b]phenanthrene & 4 & 3 \\
\hline 2,35 & $\mathrm{C}_{10 \mathrm{H}_{14}}$ & $488-23-3$ & Benzene, 1,2,3,4-tetramethyl- & 3 & 2 \\
\hline 2,33 & $\mathrm{C}_{1} 6 \mathrm{H}_{10 S}$ & $239-35-0$ & Benzo[b]naphtho[2,1-d]thiophene & 3 & 2 \\
\hline 2,29 & $\mathrm{C}_{17} \mathrm{H}_{12} \mathrm{~S}$ & - & 2-Methylbenzo[b]naphtho[2,1-d]thiophene & 3 & 3 \\
\hline 2,24 & $\mathrm{C}_{6} \mathrm{H}_{11} \mathrm{NO}_{2}$ & $1696-20-4$ & Morpholine, 4-acetyl- & 1 & 3 \\
\hline 2,09 & $\mathrm{C}_{14} \mathrm{H}_{12} \mathrm{~S}$ & $1136-85-2$ & 3,7-Dimethyldibenzothiophene & 3 & 2 \\
\hline 1,94 & $\mathrm{C}_{2} \mathrm{OH}_{14}$ & $612-78-2$ & 2,2'-Binaphthalene & 3 & 3 \\
\hline 1,78 & $\mathrm{C}_{2} \mathrm{OH}_{32} \mathrm{O}_{2}$ & - & Benzoic acid, tridecyl ester & 4 & 3 \\
\hline 1,74 & $\mathrm{C}_{19} \mathrm{H}_{4} \mathrm{OO} 25 \mathrm{Si}$ & $55520-89-3$ & Hexadecanoic acid, trimethylsilyl ester & 6 & 2 \\
\hline 1,72 & $\mathrm{C}_{14} \mathrm{H}_{14}$ & $613-33-2$ & 4,4'-Dimethylbiphenyl & 3 & 2 \\
\hline 1,70 & $\mathrm{C}_{12} \mathrm{H}_{12} \mathrm{O}_{5}$ & $5062-30-6$ & Phenacylidene diacetate & 2 & 2 \\
\hline 1,63 & $\mathrm{C}_{1} 6 \mathrm{H}_{11} \mathrm{~N}$ & - & 11H-Benzo[a]carbazole (isomer) & 7 & 3 \\
\hline 1,55 & $\mathrm{C}_{22} \mathrm{H}_{3} 6 \mathrm{O}_{2}$ & - & Benzoic acid, pentadecyl ester & 5 & 3 \\
\hline 1,54 & $\mathrm{C}_{1} 8 \mathrm{H}_{2} 8 \mathrm{O}_{2}$ & $6316-30-9$ & Benzoic acid, undecyl ester & 5 & 3 \\
\hline 1,52 & $\mathrm{C} 8 \mathrm{H}_{8} \mathrm{O}_{2}$ & $582-24-1$ & Ethanone, 2-hydroxy-1-phenyl- & 3 & 2 \\
\hline 1,07 & $\mathrm{C}_{15} \mathrm{H}_{22} \mathrm{O}_{3}$ & - & Salicylic acid, butyl ether, butyl ester & 3 & 2 \\
\hline \multirow[t]{16}{*}{0,89} & $\mathrm{C}_{13} \mathrm{H}_{12}$ & $643-93-6$ & 1,1'-Biphenyl, 3-methyl- & 3 & 2 \\
\hline & $\mathrm{C}_{13} \mathrm{H}_{16} \mathrm{O}_{5}$ & $99469-99-5$ & ethyl 2-ethoxy-4-carboxymethylbenzoate & 2 & 2 \\
\hline & & $17202-41-4$ & PFNS & 1 & 2 \\
\hline & $\mathrm{C}_{12} \mathrm{HF}_{23} \mathrm{O}_{2}$ & $307-55-1$ & PFDOA & 1 & 2 \\
\hline & & $92612-52-7$ & PFHXA & 4 & 2 \\
\hline & & $39108-34-4$ & $8: 2$ FTS & 1 & 2 \\
\hline & & $355-46-4$ & $\mathrm{PFH} \times \mathrm{S}$ & 1 & 2 \\
\hline & $\mathrm{C}_{9} \mathrm{H}_{6} \mathrm{~F}_{12} \mathrm{O}$ & 78971-81-o & & 1 & 2 \\
\hline & & 40143-77-9 & C6/C6-PFPIA & 1 & 2 \\
\hline & $\mathrm{C}_{18} \mathrm{H}_{11} \mathrm{~F}_{19} \mathrm{O}_{2}$ & $94159-92-9$ & & 1 & 2 \\
\hline & & $1478-61-1$ & hexafluoroBPA (bisfenol AF) & 1 & 1 \\
\hline & & $843-55-0$ & bisfenol Z & 3 & 1 \\
\hline & & $13595-25-0$ & bisphenol M & 3 & 1 \\
\hline & & $5835-26-7$ & isopimaric acid & 4 & 2 \\
\hline & & $123-08-0$ & 4-hydroxybenzaldehyde & 7 & 2 \\
\hline & & $1074-86-8$ & 4-formyl indole & 6 & 2 \\
\hline
\end{tabular}


Table 19: Sediment Sweden

\begin{tabular}{|c|c|c|c|c|c|}
\hline $\begin{array}{l}\text { Conc } \\
\text { (ng/g) }\end{array}$ & $\begin{array}{l}\text { Molecular } \\
\text { Formula }\end{array}$ & CAS & Common name & $\begin{array}{l}\text { Occur/ } \\
\text { matrix }\end{array}$ & $\begin{array}{r}\text { ID } \\
\text { Level }\end{array}$ \\
\hline 1594,44 & $\mathrm{C}_{2} \mathrm{OH}_{12}$ & $205-99-2$ & Benzo[b]fluoranthene & 4 & 2 \\
\hline 1108,95 & $\mathrm{C}_{1} 6 \mathrm{H}_{10}$ & $206-44-0$ & Fluoranthene & 6 & 2 \\
\hline 811,12 & $\mathrm{C}_{22} \mathrm{H}_{12}$ & $193-39-5$ & Indeno[ $[1,2,3-c d]$ pyrene & 4 & 2 \\
\hline 755,77 & $\mathrm{C}_{2} \mathrm{OH}_{12}$ & $192-97-2$ & Benzo[e]pyrene & 5 & 2 \\
\hline 711,73 & $\mathrm{C}_{2} \mathrm{OH}_{12}$ & $50-32-8$ & Benzo[a]pyrene & 5 & 2 \\
\hline 623,82 & $\mathrm{C}_{18} \mathrm{H}_{12}$ & $217-59-4$ & Triphenylene & 6 & 2 \\
\hline 603,09 & $\mathrm{C}_{3} \mathrm{OH}_{5} \mathrm{O}$ & $1615-91-4$ & Diploptene & 4 & 3 \\
\hline 471,01 & $\mathrm{C}_{18} \mathrm{H}_{12}$ & $56-55-3$ & Benz[a]anthracene & 6 & 2 \\
\hline 391,03 & $\mathrm{C}_{3} \mathrm{OH}_{48} \mathrm{O}$ & $1617-70-5$ & Lupenone & 2 & 3 \\
\hline 389,23 & $\mathrm{C}_{9} \mathrm{H}_{12} \mathrm{O}_{2}$ & $1125-21-9$ & 2,6,6-Trimethyl-2-cyclohexene-1,4-dione & 6 & 2 \\
\hline 363,59 & $\mathrm{C}_{12} \mathrm{H}_{12}$ & - & Naphthalene, dimethyl- & 10 & 3 \\
\hline 266,21 & $\mathrm{C}_{1} 8 \mathrm{H}_{18}$ & $66552-97-4$ & 2-Isopropyl-10-methylphenanthrene & 5 & 3 \\
\hline 244,63 & $\mathrm{C}_{2} \mathrm{OH}_{12}$ & $198-55-0$ & Perylene & 5 & 2 \\
\hline 236,31 & $\mathrm{C}_{11} \mathrm{H}_{16 \mathrm{O}_{2}}$ & $15356-74-8$ & $2(4 \mathrm{H})$-Benzofuranone, $5,6,7,7$ a-tetrahydro- $4,4,7$ a-trimethyl- & 5 & 2 \\
\hline 227,71 & $\mathrm{C}_{1} 8 \mathrm{H}_{22}$ & $6566-19-4$ & 10,18-Bisnorabieta-5,7,9(10),11,13-pentaene & 3 & 3 \\
\hline 226,99 & $\mathrm{C}_{13} \mathrm{H}_{16} \mathrm{O}$ & 55591-10-1 & Ethanone, 1-(2,3-dihydro-1,1-dimethyl-1H-inden-4-yl)- & 4 & 3 \\
\hline 194,71 & $\mathrm{C}_{19} \mathrm{H}_{14}$ & $82979-72-4$ & 8,9-Dihydro-7H-cyclopenta[a]pyrene & 5 & 3 \\
\hline 179,94 & $\mathrm{C}_{17} \mathrm{H}_{12}$ & $199-94-0$ & $7 \mathrm{H}$-Benzo[de]anthracene & 4 & 3 \\
\hline $179,5^{2}$ & $\mathrm{C}_{21} \mathrm{H}_{14}$ & $63104-32-5$ & 10-Methylbenzo(a)pyrene & 4 & 3 \\
\hline 175,34 & $\mathrm{C}_{14} \mathrm{H}_{10}$ & $120-12-7$ & Anthracene & 6 & 2 \\
\hline 175,25 & $\mathrm{C}_{2} \mathrm{OH}_{12}$ & $205-82-3$ & Benzo[j]fluoranthene & 4 & 2 \\
\hline 153,13 & $\mathrm{C}_{22} \mathrm{H}_{34} \mathrm{O}_{4}$ & - & Phthalic acid, nonyl 2-pentyl ester & 2 & 2 \\
\hline 127,93 & $\mathrm{C}_{17} \mathrm{H}_{12}$ & - & $7 \mathrm{H}$-Benzo[c]fluorene isomer & 2 & 3 \\
\hline 126,15 & $\mathrm{C}_{1} 6 \mathrm{H}_{10 S}$ & $205-43-6$ & Benzo[b]naphtho[1,2-d]thiophene & 5 & 2 \\
\hline 116,02 & $\mathrm{C}_{13} \mathrm{H}_{2} \mathrm{OO}$ & $79-77-6$ & trans-á-lonone & 1 & 2 \\
\hline 115,30 & $\mathrm{C}_{21} \mathrm{H}_{14}$ & $241-28-1$ & $8 \mathrm{H}$-Indeno[2,1-b]phenanthrene & 4 & 3 \\
\hline 110,32 & $\mathrm{C}_{1} 6 \mathrm{H}_{10} \mathrm{O}$ & $205-39-0$ & Benzo(b)naphtho(1,2-d)furan & 5 & 3 \\
\hline 100,41 & $\mathrm{C}_{2} \mathrm{OH}_{16}$ & $35187-19-0$ & 4,12-Dimethylbenz[a]anthracene & 4 & 3 \\
\hline 86,84 & $\mathrm{C}_{7} \mathrm{H}_{9} \mathrm{NO}_{2}$ & $20189-42-8$ & 1H-Pyrrole-2,5-dione, 3-ethyl-4-methyl- & 7 & 3 \\
\hline 78,80 & $\mathrm{C}_{17} \mathrm{H}_{16}$ & $3674-73-5$ & Phenanthrene, 2,3,5-trimethyl- & 4 & 2 \\
\hline 77,44 & $\mathrm{C}_{21} \mathrm{H}_{14}$ & $239-85-0$ & ${ }_{13} \mathrm{H}$-Dibenzo[a, $\left.\mathrm{h}\right]$ fluorene & 5 & 3 \\
\hline 75,94 & $\mathrm{C}_{15} \mathrm{H}_{12}$ & $832-69-9$ & Phenanthrene, 1-methyl- & 3 & 2 \\
\hline 68,22 & $\mathrm{C}_{18} 8 \mathrm{H}_{12}$ & $195-19-7$ & Benzo[c]phenanthrene & 5 & 2 \\
\hline 65,28 & $\mathrm{C}_{21} \mathrm{H}_{3} 6$ & $641-85-0$ & Allopregnane & 3 & 3 \\
\hline 62,75 & $\mathrm{C}_{12} \mathrm{H} 8 \mathrm{O}$ & $132-64-9$ & Dibenzofuran & 5 & 2 \\
\hline 61,98 & $\mathrm{C}_{15} \mathrm{H}_{10}$ & $83469-43-6$ & $6 \mathrm{H}-\mathrm{Cyclobuta[jk]phenanthrene}$ & 5 & 2 \\
\hline 58,83 & $\mathrm{C}_{12} \mathrm{H}_{12}$ & - & Naphthalene, dimethyl- & 10 & 3 \\
\hline 58,62 & $\mathrm{C}_{16} \mathrm{H}_{14}$ & $3674-69-9$ & Phenanthrene, 4,5-dimethyl- & 5 & 2 \\
\hline 57,53 & $\mathrm{C}_{7} \mathrm{H}_{7} \mathrm{NO}_{2}$ & $21494-57-5$ & 1H-Pyrrole-2,5-dione, 3-ethenyl-4-methyl- & 6 & 3 \\
\hline 57,24 & $\mathrm{C}_{1} 6 \mathrm{H}_{10} \mathrm{O}$ & $200-23-7$ & Benzo[kl]xanthene & 4 & 3 \\
\hline 56,86 & $\mathrm{C}_{9} \mathrm{H}_{1} 8 \mathrm{Cl}_{3} \mathrm{O}_{4} \mathrm{P}$ & $13674-84-5$ & 2-Propanol, 1-chloro-, phosphate (3:1) & 6 & 2 \\
\hline 55,56 & $\mathrm{C}_{17} \mathrm{H}_{12}$ & - & 7H-Benzo[c]fluorene (isomer) & 13 & 3 \\
\hline 55,39 & $\mathrm{C}_{1} 8 \mathrm{H}_{10 O}$ & $191-37-7$ & Naphtho[2,1,8,7-kImn]xanthene & 4 & 3 \\
\hline 55,35 & $\mathrm{C}_{13} \mathrm{H}_{10}$ & $86-73-7$ & Fluorene & 4 & 2 \\
\hline 53,15 & $\mathrm{C}_{18} \mathrm{H}_{10}$ & $203-12-3$ & Benzo[ghi]fluoranthene & 3 & 2 \\
\hline 52,75 & $\mathrm{C}_{1} 6 \mathrm{H}_{12}$ & $6232-48-0$ & Acephenanthrylene, 4,5-dihydro- & 5 & 2 \\
\hline 46,53 & $\mathrm{C}_{16} 6 \mathrm{H}_{14}$ & $1576-67-6$ & Phenanthrene, 3,6-dimethyl- & 5 & 2 \\
\hline 45,40 & $\mathrm{C}_{1} 6 \mathrm{H}_{11} \mathrm{~N}$ & - & $11 \mathrm{H}$-Benzo[a]carbazole (isomer) & 7 & 3 \\
\hline 44,67 & $\mathrm{C}_{15} \mathrm{H}_{12}$ & $832-71-3$ & Phenanthrene, 3-methyl- & 4 & 2 \\
\hline 44,44 & $\mathrm{C}_{1} 6 \mathrm{H}_{16}$ & $7694-30-6$ & Benzene, $1,1^{\prime}-(1,2$-cyclobutanediyl)bis-, cis- & 5 & 2 \\
\hline 37,75 & $\mathrm{C}_{11} \mathrm{H}_{10}$ & $91-57-6$ & Naphthalene, 2-methyl- & 6 & 2 \\
\hline 36,84 & $\mathrm{C}_{13} \mathrm{H}_{10 O}$ & $3218-36-8$ & [1,1'-Biphenyl]-4-carboxaldehyde & 4 & 2 \\
\hline 36,79 & $\mathrm{C}_{17} \mathrm{H}_{12}$ & $243-17-4$ & $11 \mathrm{H}-$ Benzo[b]fluorene & 5 & 2 \\
\hline 36,77 & $\mathrm{C}_{17} \mathrm{H}_{12} \mathrm{~S}$ & - & 2-Methylbenzo[b]naphtho[2,1-d]thiophene & 3 & 3 \\
\hline 34,41 & $\mathrm{C}_{14} \mathrm{H}_{12} \mathrm{~S}$ & $1136-85-2$ & 3,7-Dimethyldibenzothiophene & 3 & 2 \\
\hline 34,27 & $\mathrm{C}_{1} 6 \mathrm{H}_{10 S}$ & $227-86-1$ & Anthra(1,2-b)thiophene & 3 & 3 \\
\hline 32,43 & $\mathrm{C}_{18} \mathrm{H}_{14} \mathrm{~S}$ & $24964-12-3$ & Benzo[b]naphtho[2,3-d]thiophene, 7,8-dimethyl- & 3 & 3 \\
\hline 32,04 & $\mathrm{C}_{17} \mathrm{H}_{12}$ & - & 7H-Benzo[c]fluorene (isomer) & 13 & 3 \\
\hline 30,67 & $\mathrm{C}_{1} 6 \mathrm{H}_{11} \mathrm{~N}$ & $243-28-7$ & Benzo(b)carbazole & 2 & 2 \\
\hline 29,34 & $\mathrm{C}_{16} 6 \mathrm{H}_{10 O}$ & $243-42-5$ & Benzo[b]naphtho[2,3-d]furan & 4 & 3 \\
\hline 29,09 & $\mathrm{C}_{17} \mathrm{H}_{11} \mathrm{~N}$ & $225-51-4$ & Benz $[c]$ acridine & 2 & 3 \\
\hline 25,68 & $\mathrm{C}_{17} \mathrm{H}_{10 O}$ & $82-05-3$ & $7 \mathrm{H}$-Benz[de]anthracen-7-one & 4 & 2 \\
\hline $25,5^{8}$ & $\mathrm{C}_{1} 6 \mathrm{H}_{11} \mathrm{~N}$ & - & $11 \mathrm{H}-$ Benzo[a]carbazole (isomer) & 7 & 3 \\
\hline
\end{tabular}


Continued

\begin{tabular}{|c|c|c|c|c|c|}
\hline $\begin{array}{l}\text { Conc } \\
(\mathrm{ng} / \mathrm{g})\end{array}$ & $\begin{array}{l}\text { Molecular } \\
\text { Formula }\end{array}$ & CAS & Common name & $\begin{array}{l}\text { Occur/ } \\
\text { matrix }\end{array}$ & $\begin{array}{r}\text { ID } \\
\text { Level }\end{array}$ \\
\hline 24,40 & $\mathrm{C}_{15} \mathrm{H}_{12}$ & $832-64-4$ & Phenanthrene, 4-methyl- & 4 & 2 \\
\hline 21,60 & $\mathrm{C}_{2} \mathrm{OH}_{14}$ & $612-78-2$ & 2,2'-Binaphthalene & 3 & 3 \\
\hline 20,47 & $\mathrm{C}_{1} 6 \mathrm{H}_{10 S}$ & $239-35-0$ & Benzo[b]naphtho[2,1-d]thiophene & 3 & 2 \\
\hline 18,21 & $\mathrm{C}_{15} \mathrm{H} 8 \mathrm{O}$ & $5737-13-3$ & Cyclopenta(def)phenanthrenone & 4 & 2 \\
\hline 18,15 & $\mathrm{C}_{14} \mathrm{H}_{8} \mathrm{O}_{2}$ & $84-65-1$ & 9,10 -Anthracenedione & 5 & 2 \\
\hline 17,49 & $\mathrm{C}_{19} \mathrm{H}_{12}$ & 50861-05-7 & 9H-Cyclopenta[a]pyrene & 5 & 2 \\
\hline 16,29 & $\mathrm{C}_{13} \mathrm{H}_{10} \mathrm{O}$ & $92-83-1$ & $9 \mathrm{H}$-Xanthene & 5 & 2 \\
\hline 15,39 & $\mathrm{C}_{9} \mathrm{H}_{7} \mathrm{NO}$ & $487-89-8$ & $1 \mathrm{H}$-Indole-3-carboxaldehyde & 3 & 2 \\
\hline 15,06 & $\mathrm{C}_{13} \mathrm{H} 8 \mathrm{O}$ & $548-39-0$ & $1 \mathrm{H}$-Phenalen-1-one & 4 & 2 \\
\hline 12,07 & $\mathrm{C}_{9} \mathrm{H}_{14} \mathrm{O} 6$ & $102-76-1$ & Triacetin & 3 & 2 \\
\hline 9,18 & $\mathrm{C}_{11} \mathrm{H}_{20}$ & - & trans-Decalin, 2-methyl- & 1 & 2 \\
\hline 8,97 & $\mathrm{C} 8 \mathrm{H}_{8} \mathrm{O}_{3}$ & $121-33-5$ & Vanillin & 2 & 2 \\
\hline 8,84 & $\mathrm{C} 8 \mathrm{H}_{1} 6 \mathrm{O}$ & $5715-23-1$ & 3,4-Dimethylcyclohexanol & 2 & 2 \\
\hline 8,84 & $\mathrm{C}_{12} \mathrm{H}_{22} \mathrm{O}_{2}$ & $1900-69-2$ & Cyclohexanol, 4-(1,1-dimethylethyl)-, acetate, trans- & 1 & 2 \\
\hline 8,69 & $\mathrm{C}_{2} 4 \mathrm{H}_{3} 8 \mathrm{O}_{4}$ & $6422-86-2$ & 1,4-Benzenedicarboxylic acid, bis(2-ethylhexyl) ester & 2 & 3 \\
\hline 7,88 & $\mathrm{C}_{1} \mathrm{OH}_{16}$ & $3760-14-3$ & 1,5-Cyclooctadiene, 1,5-dimethyl- & 4 & 3 \\
\hline 7,79 & $\mathrm{C}_{12} \mathrm{H}_{22}$ & $1008-80-6$ & Naphthalene, decahydro-2,3-dimethyl- & 1 & 3 \\
\hline 6,97 & $\mathrm{C}_{12} \mathrm{H}_{24} \mathrm{O}_{3}$ & $74367-34-3$ & Propanoic acid, 2-methyl-, 3-hydroxy-2,4,4-trimethylpentyl ester:2 & 1 & 2 \\
\hline 6,91 & $\mathrm{C}_{8} \mathrm{H}_{10 O}$ & $122-99-6$ & Ethanol, 2-phenoxy- & 3 & 2 \\
\hline 6,9 & $\mathrm{C}_{14} \mathrm{H}_{14} \mathrm{O}$ & $19578-70-2$ & Benzene, 1-methyl-2-(phenylmethoxy)- & 5 & 2 \\
\hline 6,86 & $\mathrm{C}_{6} \mathrm{H}_{5} \mathrm{Cl}_{2} \mathrm{~N}$ & $608-27-5$ & Benzenamine, 2,3-dichloro- & 1 & 2 \\
\hline 6,74 & $\mathrm{C}_{15} \mathrm{H}_{28}$ & $54832-83-6$ & $1 \mathrm{H}$-Indene, octahydro-2,2,4,4,7,7-hexamethyl-, trans- & 3 & 3 \\
\hline 6,66 & $\mathrm{C}_{10 \mathrm{H}_{14}}$ & $527-53-7$ & Benzene, 1,2,3,5-tetramethyl- & 2 & 2 \\
\hline 6,32 & $\mathrm{C}_{9} \mathrm{H}_{10} \mathrm{O}_{2}$ & $101-41-7$ & Phenylacetic acid, methyl ester & 2 & 2 \\
\hline 6,19 & $\mathrm{C}_{1} 8 \mathrm{H}_{3} \mathrm{O}$ & $2719-62-2$ & Benzene, (1-pentylheptyl)- & 4 & 2 \\
\hline 6,11 & $\mathrm{C}_{17} \mathrm{H}_{28}$ & 4537-15-9 & Benzene, (1-butylheptyl)- & 3 & 2 \\
\hline 6,04 & $\mathrm{C}_{11} \mathrm{H}_{20 O}$ & $104-67-6$ & $2(3 \mathrm{H})$-Furanone, 5-heptyldihydro- & 1 & 2 \\
\hline 5,93 & $\mathrm{C}_{12} \mathrm{H}_{22}$ & $66660-41-1$ & 2-Ethyldecahydronaphthalene, $(Z, E)$ - & 1 & 2 \\
\hline 5,9 & $\mathrm{C}_{15} \mathrm{H}_{24}$ & $242794-76-9$ & Bicyclo[5.2.0]nonane, 2-methylene-4,8,8-trimethyl-4-vinyl- & 3 & 2 \\
\hline 5,76 & $\mathrm{C}_{13} \mathrm{H}_{16}$ & $30364-38-6$ & Naphthalene, 1,2-dihydro-1,1,6-trimethyl- & 1 & 2 \\
\hline 5,58 & $\mathrm{C}_{1} 8 \mathrm{H}_{2} 8 \mathrm{O}_{2}$ & $6316-30-9$ & Benzoic acid, undecyl ester & 5 & 3 \\
\hline 5,53 & $\mathrm{C}_{22} \mathrm{H}_{3} 6 \mathrm{O}_{2}$ & - & Benzoic acid, pentadecyl ester & 5 & 3 \\
\hline 5,47 & $\mathrm{C}_{6} \mathrm{H}_{4} \mathrm{Cl}_{3} \mathrm{~N}$ & $634-91-3$ & Benzenamine, 3,4,5-trichloro- & 1 & 2 \\
\hline 5,47 & $\mathrm{C}_{10 \mathrm{H}_{12} \mathrm{O}}$ & $487-68-3$ & Benzaldehyde, 2,4,6-trimethyl- & 3 & 3 \\
\hline 5,15 & $\mathrm{Cg} \mathrm{H} 8 \mathrm{O}$ & $83-33-0$ & 1H-Inden-1-one, 2,3-dihydro- & 1 & 2 \\
\hline 4,91 & $\mathrm{C}_{11} \mathrm{H}_{10}$ & $90-12-0$ & Naphthalene, 1-methyl- & 6 & 2 \\
\hline 4,84 & $\mathrm{C}_{7} \mathrm{H}_{5} \mathrm{NS}$ & $95-16-9$ & Benzothiazole & 6 & 2 \\
\hline 4,83 & $\mathrm{C}_{15} \mathrm{H}_{22} \mathrm{O}_{3}$ & - & Salicylic acid, butyl ether, butyl ester & 3 & 2 \\
\hline 4,76 & $\mathrm{C}_{14} \mathrm{H}_{14}$ & $613-33-2$ & 4,4'-Dimethylbiphenyl & 3 & 2 \\
\hline 4,72 & $\mathrm{Cg} \mathrm{HgN}_{9}$ & $83-34-1$ & $1 \mathrm{H}$-Indole, 3-methyl- & 1 & 2 \\
\hline 4,6 & $\mathrm{C}_{19} \mathrm{H}_{4} \mathrm{OO} 2 \mathrm{Si}$ & $55520-89-3$ & Hexadecanoic acid, trimethylsilyl ester & 6 & 2 \\
\hline 4,54 & $\mathrm{C} 8 \mathrm{H}_{7} \mathrm{~N}$ & $120-72-9$ & Indole & 2 & 2 \\
\hline 4,48 & $\mathrm{C} 8 \mathrm{H}_{8} \mathrm{O}_{2}$ & $582-24-1$ & Ethanone, 2-hydroxy-1-phenyl- & 3 & 2 \\
\hline 4,41 & $\mathrm{C}_{11} \mathrm{H}_{14} \mathrm{O}_{4}$ & & 1-Methyl-1,3-cyclohexadiene-5,6-diol diacetate & 3 & 3 \\
\hline 4,27 & $\mathrm{C}_{15} \mathrm{H}_{22}$ & $483-77-2$ & Naphthalene, 1,2,3,4-tetrahydro-1,6-dimethyl-4-(1-methylethyl)-, (1S-cis)- & 3 & 2 \\
\hline 4,27 & $\mathrm{C}_{13} \mathrm{H}_{18}$ & $30316-36-0$ & Naphthalene, 1,2,3,4-tetrahydro-1,6,8-trimethyl- & 1 & 2 \\
\hline 4,14 & $\mathrm{C}_{10 \mathrm{O} 8}$ & $91-20-3$ & Naphthalene & 4 & 2 \\
\hline 4,1 & $\mathrm{C}_{11} \mathrm{H}_{18} \mathrm{O}_{4}$ & - & Fumaric acid, ethyl 3-methylbut-2-yl ester & 3 & 2 \\
\hline 3,87 & $\mathrm{C}_{13} \mathrm{H}_{10 O}$ & $119-61-9$ & Benzophenone & 7 & 2 \\
\hline 3,85 & $\mathrm{C}_{7} \mathrm{H}_{4} \mathrm{CINO}$ & $2909-38-8$ & Benzene, 1-chloro-3-isocyanato- & 1 & 3 \\
\hline 3,77 & $\mathrm{C}_{1} 8 \mathrm{H}_{2} 6 \mathrm{O}$ & $1222-05-5$ & Galaxolide & 2 & 2 \\
\hline 3,73 & $\mathrm{C}_{13} \mathrm{H}_{12}$ & $643-93-6$ & 1,1'-Biphenyl, 3-methyl- & 3 & 2 \\
\hline 3,54 & $\mathrm{C}_{10 \mathrm{H}_{14}}$ & $488-23-3$ & Benzene, 1,2,3,4-tetramethyl- & 3 & 2 \\
\hline 3,2 & $\mathrm{C}_{12} \mathrm{H}_{6} \mathrm{Cl}_{4}$ & $35693-99-3$ & 1,1'-Biphenyl, 2,2',5,5'-tetramethyl- & 6 & 3 \\
\hline 2,91 & $\mathrm{C}_{12} \mathrm{H}_{6} \mathrm{Cl}_{4}$ & $35693-99-3$ & 1,1'-Biphenyl, 2,2',5,5'-tetramethyl- (isomer) & 3 & 3 \\
\hline 2,89 & $\mathrm{C}_{15} \mathrm{H}_{12}$ & $27410-55-5$ & 8,9-Dihydrocyclopenta[def]phenanthrene & 5 & 3 \\
\hline 2,86 & $\mathrm{C}_{8} \mathrm{H}_{7} \mathrm{NO}$ & $2380-94-1$ & ${ }_{1} \mathrm{H}$-Indol-4-ol & 2 & 3 \\
\hline 2,69 & $\mathrm{C}_{3} \mathrm{OH}_{4} 8 \mathrm{O}$ & - & $\begin{array}{l}\text { 4,4,6a,6b,8a,11,11,14b-Octamethyl-1,4,4a,5,6,6a,6b,7,8,8a,9,10,11,12, } \\
12 \mathrm{a}, 14,14 \mathrm{a}, 14 \mathrm{~b} \text {-octadecahydro-2H-picen-3-one }\end{array}$ & 2 & 3 \\
\hline 2,44 & $\mathrm{C}_{13} \mathrm{H}_{2} \mathrm{OO}$ & $14901-07-6$ & 3-Buten-2-one, 4-(2,6,6-trimethyl-1-cyclohexen-1-yl)- & 3 & 2 \\
\hline 2,18 & $\mathrm{C}_{13} \mathrm{H}_{14}$ & $829-26-5$ & Naphthalene, 2,3,6-trimethyl- & 4 & 2 \\
\hline 1,24 & $\mathrm{C}_{22} \mathrm{H}_{12}$ & $193-43-1$ & Indeno[1,2,3-cd]fluoranthene & 4 & 2 \\
\hline 1,23 & $\mathrm{C}_{1} 6 \mathrm{H}_{10}$ & $129-00-0$ & Pyrene & 6 & 2 \\
\hline 1,08 & $\mathrm{C}_{23} \mathrm{H}_{3} 6 \mathrm{O}_{4}$ & - & Phthalic acid, hept-3-yl octyl ester & 6 & 2 \\
\hline
\end{tabular}


Continued

\begin{tabular}{|c|c|c|c|c|c|}
\hline $\begin{array}{l}\text { Conc } \\
\text { (ng/g) }\end{array}$ & $\begin{array}{l}\text { Molecular } \\
\text { Formula }\end{array}$ & CAS & Common name & $\begin{array}{l}\text { Occur/ } \\
\text { matrix }\end{array}$ & $\begin{array}{r}\text { ID } \\
\text { Level }\end{array}$ \\
\hline$-0,58$ & $\mathrm{C}_{12} \mathrm{H}_{5} \mathrm{Cl}_{5}$ & $32598-14-4$ & 1,1'-Biphenyl, 2,3,3',4,4'-pentachloro- & 2 & 2 \\
\hline \multirow[t]{7}{*}{$-0,96$} & $\mathrm{C}_{14} \mathrm{H}_{10}$ & $85-01-8$ & Phenanthrene & 6 & 2 \\
\hline & & $4247-02-3$ & isobutyl 4-hydroxybenzoate & 1 & 2 \\
\hline & & $99469-99-5$ & ethyl 2-ethoxy-4-carboxymethylbenzoate & 2 & 2 \\
\hline & & $92612-52-7$ & PFH $\times A$ & 4 & 2 \\
\hline & & $106-44-5$ & p-cresol & 1 & 2 \\
\hline & & $123-08-0$ & 4-hydroxybenzaldehyde & 7 & 2 \\
\hline & & $1074-86-8$ & 4-formyl indole & 6 & 2 \\
\hline
\end{tabular}


Table 20: Sediment Denmark

\begin{tabular}{|c|c|c|c|c|c|}
\hline $\begin{array}{l}\text { Conc } \\
\text { (ng/g) }\end{array}$ & $\begin{array}{l}\text { Molecular } \\
\text { Formula }\end{array}$ & CAS & Common name & $\begin{array}{l}\text { Occur/ } \\
\text { matrix }\end{array}$ & $\begin{array}{r}\text { ID } \\
\text { Level }\end{array}$ \\
\hline 265,66 & $\mathrm{C}_{1} 6 \mathrm{H}_{10}$ & $206-44-0$ & Fluoranthene & 6 & 2 \\
\hline 204,98 & $\mathrm{C}_{1} 6 \mathrm{H}_{10}$ & $129-00-0$ & Pyrene & 6 & 2 \\
\hline 174,27 & $\mathrm{C}_{2} \mathrm{OH}_{12}$ & $207-08-9$ & Benzo[k]fluoranthene & 1 & 2 \\
\hline 166,08 & $\mathrm{C}_{2} \mathrm{OH}_{12}$ & $205-99-2$ & Benzo[b]fluoranthene & 4 & 2 \\
\hline 136,36 & $\mathrm{C}_{22} \mathrm{H}_{12}$ & $193-39-5$ & Indeno[1,2,3-cd]pyrene & 4 & 2 \\
\hline 132,12 & $\mathrm{C}_{3} \mathrm{OH}_{50}$ & $1615-91-4$ & Diploptene & 4 & 3 \\
\hline 111,81 & $\mathrm{C}_{2} \mathrm{OH}_{12}$ & $192-97-2$ & Benzo[e]pyrene & 5 & 2 \\
\hline 106,32 & $\mathrm{C}_{2} \mathrm{OH}_{12}$ & $50-32-8$ & Benzo[a]pyrene & 5 & 2 \\
\hline 92,74 & $\mathrm{C}_{1} 8 \mathrm{H}_{12}$ & $217-59-4$ & Triphenylene & 6 & 2 \\
\hline 87,77 & $\mathrm{C}_{9} \mathrm{H}_{12} \mathrm{O}_{2}$ & $1125-21-9$ & 2,6,6-Trimethyl-2-cyclohexene-1,4-dione & 6 & 2 \\
\hline 85,20 & $\mathrm{C}_{22} \mathrm{H}_{12}$ & $193-43-1$ & Indeno[1,2,3-cd]fluoranthene & 4 & 2 \\
\hline 84,02 & $\mathrm{C}_{11} \mathrm{H}_{16} 6 \mathrm{O}_{2}$ & $15356-74-8$ & $2(4 \mathrm{H})$-Benzofuranone, 5,6,7,7a-tetrahydro-4,4,7a-trimethyl- & 5 & 2 \\
\hline 82,77 & $\mathrm{C}_{1} 8 \mathrm{H}_{12}$ & $56-55-3$ & Benz[a]anthracene & 6 & 2 \\
\hline 63,89 & $\mathrm{C}_{23} \mathrm{H}_{3} 6 \mathrm{O}_{4}$ & - & Phthalic acid, hept-3-yl octyl ester & 6 & 2 \\
\hline 62,67 & $\mathrm{C}_{7} \mathrm{H}_{9} \mathrm{NO}_{2}$ & $20189-42-8$ & 1H-Pyrrole-2,5-dione, 3-ethyl-4-methyl- & 7 & 3 \\
\hline 40,26 & $\mathrm{C}_{14} \mathrm{H}_{10}$ & $85-01-8$ & Phenanthrene & 6 & 2 \\
\hline 36,01 & $\mathrm{C}_{13} \mathrm{H}_{2} \mathrm{OO}$ & $14901-07-6$ & 3-Buten-2-one, 4-(2,6,6-trimethyl-1-cyclohexen-1-yl)- & 3 & 2 \\
\hline 36,01 & $\mathrm{C}_{13} \mathrm{H}_{2} \mathrm{OO}$ & $79-77-6$ & trans-beta-lonone & 1 & 2 \\
\hline 29,89 & $\mathrm{C}_{13} \mathrm{H}_{16} \mathrm{O}$ & $55591-10-1$ & Ethanone, 1-(2,3-dihydro-1,1-dimethyl-1H-inden-4-yl)- & 4 & 3 \\
\hline 27,69 & $\mathrm{C}_{7} \mathrm{H}_{5} \mathrm{NS}$ & $95-16-9$ & Benzothiazole & 6 & 2 \\
\hline 27,31 & $\mathrm{C}_{2} \mathrm{OH}_{12}$ & $205-82-3$ & Benzo[j]fluoranthene & 4 & 2 \\
\hline 25,69 & $\mathrm{C}_{13} \mathrm{H}_{10} \mathrm{O}$ & $119-61-9$ & Benzophenone & 7 & 2 \\
\hline 25,24 & $\mathrm{C}_{1} 6 \mathrm{H}_{10 O}$ & $205-39-0$ & Benzo(b)naphtho(1,2-d)furan & 5 & 3 \\
\hline 24,98 & $\mathrm{C}_{17} \mathrm{H}_{12}$ & $199-94-0$ & $7 \mathrm{H}$-Benzo[de]anthracene & 4 & 3 \\
\hline 21,48 & $\mathrm{C}_{1} 6 \mathrm{H}_{1} 6$ & $7694-30-6$ & Benzene, 1,1'-(1,2-cyclobutanediyl)bis-, cis- & 5 & 2 \\
\hline 19,89 & $\mathrm{C}_{14} \mathrm{H}_{10}$ & $120-12-7$ & Anthracene & 6 & 2 \\
\hline 16,36 & $\mathrm{C}_{17} \mathrm{H}_{12}$ & - & $7 \mathrm{H}$-Benzo[c]fluorene isomer & 2 & 3 \\
\hline 16,34 & $\mathrm{C}_{12} \mathrm{H}_{6} \mathrm{Cl}_{4}$ & $35693-99-3$ & 1,1'-Biphenyl, 2,2',5,5'-tetramethyl- & 6 & 3 \\
\hline 14,63 & $\mathrm{C}_{11} \mathrm{H}_{14} \mathrm{O}_{4}$ & - & 1-Methyl-1,3-cyclohexadiene-5,6-diol diacetate & 3 & 3 \\
\hline 14,56 & $\mathrm{C}_{8} \mathrm{H}_{16} 6 \mathrm{O}$ & $5715-23-1$ & 3,4-Dimethylcyclohexanol & 2 & 2 \\
\hline 14,48 & $\mathrm{C}_{2} \mathrm{OH}_{12}$ & $198-55-0$ & Perylene & 5 & 2 \\
\hline 13,81 & $\mathrm{C}_{19} \mathrm{H}_{14}$ & $82979-72-4$ & 8,9-Dihydro-7H-cyclopenta[a]pyrene & 5 & 3 \\
\hline 13,66 & $\mathrm{C}_{14} \mathrm{H}_{14} \mathrm{O}$ & $19578-70-2$ & Benzene, 1-methyl-2-(phenylmethoxy)- & 5 & 2 \\
\hline 13,46 & $\mathrm{C}_{1} 6 \mathrm{H}_{10 S}$ & $205-43-6$ & Benzo[b]naphtho[1,2-d]thiophene & 5 & 2 \\
\hline 12,91 & $\mathrm{C}_{15} \mathrm{H}_{12}$ & $27410-55-5$ & 8,9-Dihydrocyclopenta[def]phenanthrene & 5 & 3 \\
\hline 12,78 & $\mathrm{C}_{17} \mathrm{H}_{12}$ & - & $7 \mathrm{H}$-Benzo[c]fluorene (isomer) & 13 & 3 \\
\hline 11,80 & $\mathrm{C}_{15} \mathrm{H}_{10}$ & $83469-43-6$ & $6 \mathrm{H}-\mathrm{Cyclobuta}[\mathrm{jk}]$ phenanthrene & 5 & 2 \\
\hline 11,15 & $\mathrm{C}_{1} 6 \mathrm{H}_{12}$ & $6232-48-0$ & Acephenanthrylene, 4,5-dihydro- & 5 & 2 \\
\hline 10,74 & $\mathrm{C}_{9} \mathrm{H}_{1} 8 \mathrm{Cl}_{3} \mathrm{O}_{4} \mathrm{P}$ & $13674-84-5$ & 2-Propanol, 1-chloro-, phosphate (3:1) & 6 & 2 \\
\hline 9,17 & $\mathrm{C}_{1} 6 \mathrm{H}_{10 O}$ & $200-23-7$ & Benzo[kl]xanthene & 4 & 3 \\
\hline 8,78 & $\mathrm{C}_{1} \mathrm{oH} 8$ & $91-20-3$ & Naphthalene & 4 & 2 \\
\hline 8,40 & $\mathrm{C}_{1} 8 \mathrm{H}_{12}$ & $195-19-7$ & Benzo[c]phenanthrene & 5 & 2 \\
\hline 8,11 & $\mathrm{C}_{17} \mathrm{H}_{10 O}$ & $82-05-3$ & $7 \mathrm{H}$-Benz[de]anthracen-7-one & 4 & 2 \\
\hline 7,94 & $\mathrm{C}_{21} \mathrm{H}_{14}$ & $239-85-0$ & 13H-Dibenzo[a,h]fluorene & 5 & 3 \\
\hline 7,50 & $\mathrm{C}_{1} 6 \mathrm{H}_{10 O}$ & $243-42-5$ & Benzo[b]naphtho[2,3-d]furan & 4 & 3 \\
\hline 7,41 & $\mathrm{C}_{15} \mathrm{H}_{12}$ & $832-69-9$ & Phenanthrene, 1-methyl- & 3 & 2 \\
\hline 7,36 & $\mathrm{C}_{17} \mathrm{H}_{12}$ & $243-17-4$ & 11H-Benzo[b]fluorene & 5 & 2 \\
\hline 7,35 & $\mathrm{C}_{15} \mathrm{H}_{12}$ & $832-71-3$ & Phenanthrene, 3-methyl- & 4 & 2 \\
\hline 7,30 & $\mathrm{C}_{21} \mathrm{H}_{14}$ & $241-28-1$ & $8 \mathrm{H}$-Indeno[2,1-b]phenanthrene & 4 & 3 \\
\hline 6,83 & $\mathrm{C}_{12} \mathrm{H} 8 \mathrm{O}$ & $132-64-9$ & Dibenzofuran & 5 & 2 \\
\hline 6,27 & $\mathrm{C}_{17} \mathrm{H}_{11} \mathrm{~N}$ & $225-51-4$ & Benz $[c]$ acridine & 2 & 3 \\
\hline 6,02 & $\mathrm{C}_{17} \mathrm{H}_{12}$ & - & 7H-Benzo[c]fluorene (isomer) & 13 & 3 \\
\hline 5,75 & $\mathrm{C}_{12} \mathrm{H}_{24} \mathrm{O}_{3}$ & $74367-34-3$ & Propanoic acid, 2-methyl-, 3-hydroxy-2,4,4-trimethylpentyl ester & 5 & 2 \\
\hline 5,57 & $\mathrm{C}_{16} \mathrm{H}_{14}$ & $3674-69-9$ & Phenanthrene, 4,5-dimethyl- & 5 & 2 \\
\hline 5,50 & $\mathrm{C}_{1} 6 \mathrm{H}_{2} \mathrm{O}$ & $24157-81-1$ & 2,6-Diisopropylnaphthalene & 3 & 2 \\
\hline 5,44 & $\mathrm{C}_{19} \mathrm{H}_{12}$ & $50861-05-7$ & 9H-Cyclopenta[a]pyrene & 5 & 2 \\
\hline 5,24 & $\mathrm{C}_{7} \mathrm{H}_{7} \mathrm{NO}_{2}$ & $21494-57-5$ & 1H-Pyrrole-2,5-dione, 3-ethenyl-4-methyl- & 6 & 3 \\
\hline 4,74 & $\mathrm{C}_{21} \mathrm{H}_{14}$ & $63104-32-5$ & 10-Methylbenzo(a)pyrene & 4 & 3 \\
\hline 4,62 & $\mathrm{C}_{11} \mathrm{H}_{10}$ & $91-57-6$ & Naphthalene, 2-methyl- & 6 & 2 \\
\hline 4,43 & $\mathrm{C}_{12} \mathrm{H}_{12}$ & - & Naphthalene, dimethyl- & 10 & 3 \\
\hline 4,19 & $\mathrm{C}_{16} \mathrm{H}_{14}$ & $1576-67-6$ & Phenanthrene, 3,6-dimethyl- & 5 & 2 \\
\hline 3,78 & $\mathrm{C}_{1} 8 \mathrm{H}_{18}$ & $66552-97-4$ & 2-Isopropyl-10-methylphenanthrene & 5 & 3 \\
\hline
\end{tabular}


Continued

\begin{tabular}{|c|c|c|c|c|c|}
\hline $\begin{array}{l}\text { Conc } \\
\text { (ng/g) }\end{array}$ & $\begin{array}{l}\text { Molecular } \\
\text { Formula }\end{array}$ & CAS & Common name & $\begin{array}{l}\text { Occur/ } \\
\text { matrix }\end{array}$ & $\begin{array}{r}\text { ID } \\
\text { Level }\end{array}$ \\
\hline 3,78 & $\mathrm{C}_{14} \mathrm{H}_{8} \mathrm{O}_{2}$ & $84-65-1$ & 9,10-Anthracenedione & 5 & 2 \\
\hline 3,67 & $\mathrm{C}_{13} \mathrm{H}_{10 O}$ & $3218-36-8$ & [1,1'-Biphenyl]-4-carboxaldehyde & 4 & 2 \\
\hline 3,50 & $\mathrm{C}_{10} \mathrm{H}_{12} \mathrm{O}$ & $487-68-3$ & Benzaldehyde, 2,4,6-trimethyl- & 3 & 3 \\
\hline 3,32 & $\mathrm{C}_{12} \mathrm{H}_{12}$ & - & Naphthalene, dimethyl- & 10 & 3 \\
\hline 3,11 & $\mathrm{C}_{11} \mathrm{H}_{10}$ & $90-12-0$ & Naphthalene, 1-methyl- & 6 & 2 \\
\hline 2,77 & $\mathrm{C}_{13} \mathrm{H}_{10} \mathrm{O}$ & $92-83-1$ & $9 \mathrm{H}-\mathrm{Xanthene}$ & 5 & 2 \\
\hline \multirow[t]{6}{*}{2,30} & $\mathrm{C}_{19} \mathrm{H}_{4} \mathrm{OO} 25 \mathrm{Si}$ & $55520-89-3$ & Hexadecanoic acid, trimethylsilyl ester & 6 & 2 \\
\hline & $\mathrm{C}_{13} \mathrm{H}_{8} \mathrm{~F}_{21} \mathrm{O}_{5} \mathrm{P}$ & $94158-70-0$ & & 1 & 2 \\
\hline & & $843-55-0$ & bisfenol Z & 3 & 1 \\
\hline & & $13595-25-0$ & bisphenol M & 3 & 1 \\
\hline & & $123-08-0$ & 4-hydroxybenzaldehyde & 7 & 2 \\
\hline & & $1074-86-8$ & 4-formyl indole & 6 & 2 \\
\hline
\end{tabular}


Table 21: Sediment Iceland

\begin{tabular}{|c|c|c|c|c|c|}
\hline $\begin{array}{l}\text { Conc } \\
\text { (ng/g) }\end{array}$ & $\begin{array}{l}\text { Molecular } \\
\text { Formula }\end{array}$ & CAS & Common name & $\begin{array}{l}\text { Occur/ } \\
\text { matrix }\end{array}$ & $\begin{array}{r}\text { ID } \\
\text { Level }\end{array}$ \\
\hline 66,60 & $\mathrm{C}_{23} \mathrm{H}_{3} 6 \mathrm{O}_{4}$ & - & Phthalic acid, hept-3-yl octyl ester & 6 & 2 \\
\hline 38,76 & $\mathrm{C}_{15} \mathrm{H}_{24}$ & $242794-76-9$ & Bicyclo[5.2.0]nonane, 2-methylene-4,8,8-trimethyl-4-vinyl- & 3 & 2 \\
\hline 37,70 & $\mathrm{C}_{13} \mathrm{H}_{10 O}$ & $119-61-9$ & Benzophenone & 7 & 2 \\
\hline 37,05 & $\mathrm{C}_{14} \mathrm{H}_{14} \mathrm{O}$ & $19578-70-2$ & Benzene, 1-methyl-2-(phenylmethoxy)- & 5 & 2 \\
\hline 15,94 & $\mathrm{C}_{7} \mathrm{H}_{5} \mathrm{NS}$ & $95-16-9$ & Benzothiazole & 6 & 2 \\
\hline 15,62 & $\mathrm{C}_{12} \mathrm{H}_{6} \mathrm{Cl}_{4}$ & $35693-99-3$ & 1,1'-Biphenyl, 2,2',5,5'-tetramethyl- & 6 & 3 \\
\hline 12,05 & $\mathrm{C}_{9} \mathrm{H}_{12} \mathrm{O}_{2}$ & $1125-21-9$ & 2,6,6-Trimethyl-2-cyclohexene-1,4-dione & 6 & 2 \\
\hline 9,94 & $\mathrm{C}_{1} 8 \mathrm{H}_{12}$ & $217-59-4$ & Triphenylene & 6 & 2 \\
\hline 8,82 & $\mathrm{C}_{6} \mathrm{H}_{5} \mathrm{BrO}$ & $591-20-8$ & Phenol, 3-bromo- & 2 & 2 \\
\hline 8,05 & $\mathrm{C} 8 \mathrm{H}_{7} \mathrm{~N}$ & $120-72-9$ & Indole & 2 & 2 \\
\hline 7,89 & $\mathrm{C}_{2} \mathrm{OH}_{32} \mathrm{O}_{2}$ & - & Benzoic acid, tridecyl ester & 4 & 3 \\
\hline 7,65 & $\mathrm{C}_{14} \mathrm{H}_{10}$ & $120-12-7$ & Anthracene & 6 & 2 \\
\hline $6,5^{8}$ & $\mathrm{C}_{9} \mathrm{H}_{1} 8 \mathrm{Cl}_{3} \mathrm{O}_{4} \mathrm{P}$ & $13674-84-5$ & 2-Propanol, 1-chloro-, phosphate (3:1) & 6 & 2 \\
\hline 6,37 & $\mathrm{C}_{14} \mathrm{H}_{10}$ & $85-01-8$ & Phenanthrene & 6 & 2 \\
\hline 6,35 & $\mathrm{C}_{1} 6 \mathrm{H}_{10}$ & $129-00-0$ & Pyrene & 6 & 2 \\
\hline 6,34 & $\mathrm{C}_{22} \mathrm{H}_{3} 6 \mathrm{O}_{2}$ & - & Benzoic acid, pentadecyl ester & 5 & 3 \\
\hline 6,33 & $\mathrm{C}_{1} 8 \mathrm{H}_{2} 8 \mathrm{O}_{2}$ & $6316-30-9$ & Benzoic acid, undecyl ester & 5 & 3 \\
\hline 6,12 & $\mathrm{C}_{1} 6 \mathrm{H}_{10}$ & $206-44-0$ & Fluoranthene & 6 & 2 \\
\hline 6,09 & $\mathrm{C}_{8} \mathrm{H}_{7} \mathrm{NO}$ & $2380-94-1$ & $1 \mathrm{H}$-Indol-4-ol & 2 & 3 \\
\hline 5,99 & $\mathrm{C}_{5} \mathrm{H}_{9} \mathrm{NO}_{2}$ & $4394-85-8$ & N-Formylmorpholine & 2 & 2 \\
\hline 5,08 & $\mathrm{C}_{7} \mathrm{H}_{9} \mathrm{NO}_{2}$ & $20189-42-8$ & 1H-Pyrrole-2,5-dione, 3-ethyl-4-methyl- & 7 & 2 \\
\hline 4,25 & $\mathrm{C}_{12} \mathrm{H}_{24} \mathrm{O}_{3}$ & $74367-34-3$ & Propanoic acid, 2-methyl-, 3-hydroxy-2,4,4-trimethylpentyl ester & 5 & 2 \\
\hline 3,27 & $\mathrm{C}_{1} 8 \mathrm{H}_{3} 0$ & $2719-62-2$ & Benzene, (1-pentylheptyl)- & 4 & 2 \\
\hline 3,06 & $\mathrm{C}_{1} 8 \mathrm{H}_{12}$ & $56-55-3$ & Benz[a]anthracene & 6 & 2 \\
\hline 2,46 & $\mathrm{C}_{11} \mathrm{H}_{10}$ & $91-57-6$ & Naphthalene, 2-methyl- & 6 & 2 \\
\hline 1,49 & $\mathrm{C}_{11} \mathrm{H}_{10}$ & $90-12-0$ & Naphthalene, 1-methyl- & 6 & 2 \\
\hline \multirow[t]{6}{*}{1,28} & $\mathrm{C}_{1} 9 \mathrm{H}_{4} 0 \mathrm{O}_{2} \mathrm{Si}$ & $55520-89-3$ & Hexadecanoic acid, trimethylsilyl ester & 6 & 2 \\
\hline & & $843-55-0$ & bisfenol Z & 3 & 1 \\
\hline & & $13595-25-0$ & bisphenol M & 3 & 1 \\
\hline & & $80-09-1$ & bisphenol S & 1 & 1 \\
\hline & & $5835-26-7$ & isopimaric acid & 4 & 2 \\
\hline & & $123-08-0$ & 4-hydroxybenzaldehyde & 7 & 2 \\
\hline
\end{tabular}


Table 22: Sediment Norway

\begin{tabular}{|c|c|c|c|c|c|}
\hline $\begin{array}{l}\text { Conc } \\
\text { (ng/g) }\end{array}$ & $\begin{array}{l}\text { Molecular } \\
\text { Formula }\end{array}$ & CAS & Common name & $\begin{array}{l}\text { Occur/ } \\
\text { matrix }\end{array}$ & $\begin{array}{r}\text { ID } \\
\text { Level }\end{array}$ \\
\hline 166,10 & $\mathrm{C}_{2} 4 \mathrm{H}_{3} 8 \mathrm{O}_{4}$ & - & Phthalic acid, di(2-propylpentyl) ester & 1 & 2 \\
\hline 164,41 & $\mathrm{C}_{1} 6 \mathrm{H}_{10}$ & $206-44-0$ & Fluoranthene & 6 & 2 \\
\hline 163,97 & $\mathrm{C}_{16} 6 \mathrm{H}_{10}$ & $129-00-0$ & Pyrene & 6 & 2 \\
\hline 149,78 & $\mathrm{C}_{8} \mathrm{H}_{10 O}$ & $122-99-6$ & Ethanol, 2-phenoxy- & 3 & 2 \\
\hline 101,70 & $\mathrm{C}_{2} \mathrm{OH}_{12}$ & $205-99-2$ & Benzo[b]fluoranthene & 4 & 2 \\
\hline 91,70 & $\mathrm{C}_{2} \mathrm{OH}_{12}$ & $192-97-2$ & Benzo[e]pyrene & 5 & 2 \\
\hline 84,46 & $\mathrm{C}_{18} \mathrm{H}_{12}$ & $217-59-4$ & Triphenylene & 6 & 2 \\
\hline 75,89 & $\mathrm{C}_{14} \mathrm{H}_{10}$ & $85-01-8$ & Phenanthrene & 6 & 2 \\
\hline 58,79 & $\mathrm{C}_{1} 8 \mathrm{H}_{12}$ & $56-55-3$ & Benz[a]anthracene & 6 & 2 \\
\hline 50,76 & $\mathrm{C}_{2} \mathrm{OH}_{12}$ & $50-32-8$ & Benzo[a]pyrene & 5 & 2 \\
\hline 39,93 & $\mathrm{C}_{2} \mathrm{OH}_{12}$ & $198-55-0$ & Perylene & 5 & 2 \\
\hline 37,88 & $\mathrm{C}_{13} \mathrm{H}_{10} \mathrm{O}$ & $119-61-9$ & Benzophenone & 7 & 2 \\
\hline 37,30 & $\mathrm{C}_{7} \mathrm{H}_{9} \mathrm{NO}_{2}$ & $20189-42-8$ & 1H-Pyrrole-2,5-dione, 3-ethyl-4-methyl- & 7 & 3 \\
\hline 24,14 & $\mathrm{C}_{7} \mathrm{H}_{7} \mathrm{NO}_{2}$ & $21494-57-5$ & 1H-Pyrrole-2,5-dione, 3-ethenyl-4-methyl- & 6 & 3 \\
\hline 23,53 & $\mathrm{C}_{14} \mathrm{H}_{10}$ & $120-12-7$ & Anthracene & 6 & 2 \\
\hline 23,12 & $\mathrm{C}_{11} \mathrm{H}_{1} 6 \mathrm{O}_{2}$ & $15356-74-8$ & $2(4 \mathrm{H})$-Benzofuranone, $5,6,7,7 \mathrm{a}$-tetrahydro-4,4,7a-trimethyl- & 5 & 2 \\
\hline 22,28 & $\mathrm{C}_{9} \mathrm{H}_{12} \mathrm{O}_{2}$ & $1125-21-9$ & 2,6,6-Trimethyl-2-cyclohexene-1,4-dione & 6 & 2 \\
\hline 21,46 & $\mathrm{C}_{15} \mathrm{H}_{12}$ & - & Phenanthrene, methyl- & 1 & 3 \\
\hline 21,43 & $\mathrm{C}_{1} 6 \mathrm{H}_{10} \mathrm{O}$ & 205-39-0 & Benzo(b)naphtho(1,2-d)furan & 5 & 3 \\
\hline 20,52 & $\mathrm{C}_{19} \mathrm{H}_{14}$ & $82979-72-4$ & 8,9-Dihydro-7H-cyclopenta[a]pyrene & 5 & 3 \\
\hline 20,21 & $\mathrm{C}_{17} \mathrm{H}_{12}$ & $243-17-4$ & $11 \mathrm{H}$-Benzo[b]fluorene & 5 & 2 \\
\hline 16,14 & $\mathrm{C}_{12} \mathrm{H} 8 \mathrm{O}$ & $132-64-9$ & Dibenzofuran & 5 & 2 \\
\hline 15,72 & $\mathrm{C}_{13} \mathrm{H}_{16} 6 \mathrm{O}$ & 55591-10-1 & Ethanone, 1-(2,3-dihydro-1,1-dimethyl-1H-inden-4-yl)- & 4 & 3 \\
\hline 14,52 & $\mathrm{C}_{1} 6 \mathrm{H}_{10 S}$ & $205-43-6$ & Benzo[b]naphtho[1,2-d]thiophene & 5 & 2 \\
\hline 13,93 & $\mathrm{C}_{1} 8 \mathrm{H}_{18}$ & $66552-97-4$ & 2-Isopropyl-10-methylphenanthrene & 5 & 3 \\
\hline 13,91 & $\mathrm{C}_{1} 6 \mathrm{H}_{1} 6$ & $7694-30-6$ & Benzene, 1,1'-(1,2-cyclobutanediyl)bis-, cis- & 5 & 2 \\
\hline 11,42 & $\mathrm{C}_{16} 6 \mathrm{H}_{12}$ & $6232-48-0$ & Acephenanthrylene, 4,5-dihydro- & 5 & 2 \\
\hline 9,83 & $\mathrm{C}_{15} \mathrm{H}_{10}$ & $83469-43-6$ & $6 \mathrm{H}-\mathrm{Cyclobuta}[\mathrm{jk}]$ phenanthrene & 5 & 2 \\
\hline 9,69 & $\mathrm{C}_{1} 6 \mathrm{H}_{10} \mathrm{O}$ & $200-23-7$ & Benzo[kl]xanthene & 4 & 3 \\
\hline 9,00 & $\mathrm{C}_{11} \mathrm{H}_{10}$ & $91-57-6$ & Naphthalene, 2-methyl- & 6 & 2 \\
\hline 8,75 & $\mathrm{C}_{16} \mathrm{H}_{14}$ & $3674-69-9$ & Phenanthrene, 4,5-dimethyl- & 5 & 2 \\
\hline 8,28 & $\mathrm{C}_{9} \mathrm{H}_{7} \mathrm{NO}$ & $487-89-8$ & 1H-Indole-3-carboxaldehyde & 3 & 2 \\
\hline 8,04 & $\mathrm{C}_{14} \mathrm{H}_{8} \mathrm{O}_{2}$ & $84-65-1$ & 9,10 -Anthracenedione & 5 & 2 \\
\hline 7,76 & $\mathrm{C}_{12} \mathrm{H}_{12}$ & - & Naphthalene, dimethyl- & 10 & 3 \\
\hline 7,63 & $\mathrm{C}_{19} \mathrm{H}_{12}$ & $50861-05-7$ & 9H-Cyclopenta[a]pyrene & 5 & 2 \\
\hline 7,54 & $\mathrm{C}_{13} \mathrm{H}_{10} \mathrm{O}$ & $3218-36-8$ & {$\left[1,1^{\prime}\right.$-Biphenyl]-4-carboxaldehyde } & 4 & 2 \\
\hline 7,47 & $\mathrm{C}_{21} \mathrm{H}_{14}$ & $239-85-0$ & ${ }_{13} \mathrm{H}$-Dibenzo[a,h]fluorene & 5 & 3 \\
\hline 6,89 & $\mathrm{C}_{13} \mathrm{H}_{10}$ & $86-73-7$ & Fluorene & 4 & 2 \\
\hline 6,13 & $\mathrm{C}_{11} \mathrm{H}_{10}$ & $90-12-0$ & Naphthalene, 1-methyl- & 6 & 2 \\
\hline 5,87 & $\mathrm{C}_{12} \mathrm{H}_{12}$ & - & Naphthalene, dimethyl- & 10 & 3 \\
\hline 5,56 & $\mathrm{C}_{15} \mathrm{H}_{12}$ & $27410-55-5$ & 8,9-Dihydrocyclopenta[def]phenanthrene & 5 & 3 \\
\hline 5,50 & $\mathrm{C}_{13} \mathrm{H} 8 \mathrm{O}$ & $548-39-0$ & $1 \mathrm{H}$-Phenalen-1-one & 4 & 2 \\
\hline 4,72 & $\mathrm{C}_{17} \mathrm{H}_{10 O}$ & $82-05-3$ & $7 \mathrm{H}$-Benz[de]anthracen-7-one & 4 & 2 \\
\hline 4,66 & $\mathrm{C}_{17} \mathrm{H}_{16} 6$ & $3674-73-5$ & Phenanthrene, 2,3,5-trimethyl- & 4 & 2 \\
\hline 4,50 & $\mathrm{C}_{15} \mathrm{H}_{12}$ & $832-64-4$ & Phenanthrene, 4-methyl- & 4 & 2 \\
\hline 4,45 & $\mathrm{C}_{13} \mathrm{H}_{10} \mathrm{O}$ & $92-83-1$ & $9 \mathrm{H}-$ Xanthene & 5 & 2 \\
\hline 4,41 & $\mathrm{C}_{17} \mathrm{H}_{12}$ & - & $7 \mathrm{H}$-Benzo[c]fluorene (isomer) & 13 & 3 \\
\hline 3,98 & $\mathrm{C}_{18} \mathrm{H}_{12}$ & $195-19-7$ & Benzo[c]phenanthrene & 5 & 2 \\
\hline 3,97 & $\mathrm{C}_{8} \mathrm{H}_{5} \mathrm{Br} 2 \mathrm{~N}$ & - & 2-Bromo-6-(3-bromo-prop-1-ynyl)-pyridine & 2 & 3 \\
\hline 3,88 & $\mathrm{C}_{1} 6 \mathrm{H}_{10 S}$ & $227-86-1$ & Anthra(1,2-b)thiophene & 3 & 3 \\
\hline 3,80 & $\mathrm{C}_{17} \mathrm{H}_{12}$ & - & 7H-Benzo[c]fluorene (isomer) & 13 & 3 \\
\hline 3,80 & $\mathrm{C}_{15} \mathrm{H} 8 \mathrm{O}$ & $5737-13-3$ & Cyclopenta(def)phenanthrenone & 4 & 2 \\
\hline 3,54 & $\mathrm{C}_{1} 8 \mathrm{H}_{10} \mathrm{O}$ & $191-37-7$ & Naphtho[2,1,8,7-klmn]xanthene & 4 & 3 \\
\hline 3,49 & $\mathrm{C}_{8} \mathrm{H}_{7} \mathrm{NS}_{2}$ & $615-22-5$ & Benzothiazole, 2-(methylthio)- & 2 & 2 \\
\hline 3,24 & $\mathrm{C}_{2} \mathrm{OH}_{14}$ & $612-78-2$ & 2,2'-Binaphthalene & 3 & 3 \\
\hline 3,18 & $\mathrm{C}_{13} \mathrm{H}_{14}$ & $829-26-5$ & Naphthalene, 2,3,6-trimethyl- & 4 & 2 \\
\hline 2,87 & $\mathrm{C}_{16} \mathrm{H}_{14}$ & $1576-67-6$ & Phenanthrene, 3,6-dimethyl- & 5 & 2 \\
\hline 2,77 & $\mathrm{C}_{1} 6 \mathrm{H}_{11} \mathrm{~N}$ & - & $11 \mathrm{H}-$ Benzo[a]carbazole (isomer) & 7 & 3 \\
\hline \multirow[t]{2}{*}{2,60} & $\mathrm{C}_{1} 6 \mathrm{H}_{10 S}$ & $239-35-0$ & Benzo[b]naphtho[2,1-d]thiophene & 3 & 2 \\
\hline & & $92612-52-7$ & PFHXA & 4 & 2 \\
\hline
\end{tabular}


Continued

\begin{tabular}{|c|c|c|c|c|c|}
\hline $\begin{array}{l}\text { Conc } \\
\text { (ng/g) }\end{array}$ & $\begin{array}{l}\text { Molecular } \\
\text { Formula }\end{array}$ & CAS & Common name & $\begin{array}{l}\text { Occur/ } \\
\text { matrix }\end{array}$ & $\begin{array}{r}\text { ID } \\
\text { Level }\end{array}$ \\
\hline & & $754-91-6$ & FOSA & 1 & 2 \\
\hline & & $135984-67-7$ & 4:2 FTAL & 1 & 2 \\
\hline & & $620-92-8$ & $4,4-B P F$ & 1 & 2 \\
\hline & & $5835-26-7$ & isopimaric acid & 4 & 2 \\
\hline & & $123-08-0$ & 4-hydroxybenzaldehyde & 7 & 2 \\
\hline & & $1074-86-8$ & 4-formyl indole & 6 & 2 \\
\hline
\end{tabular}


Table 23: Sediment Greenland

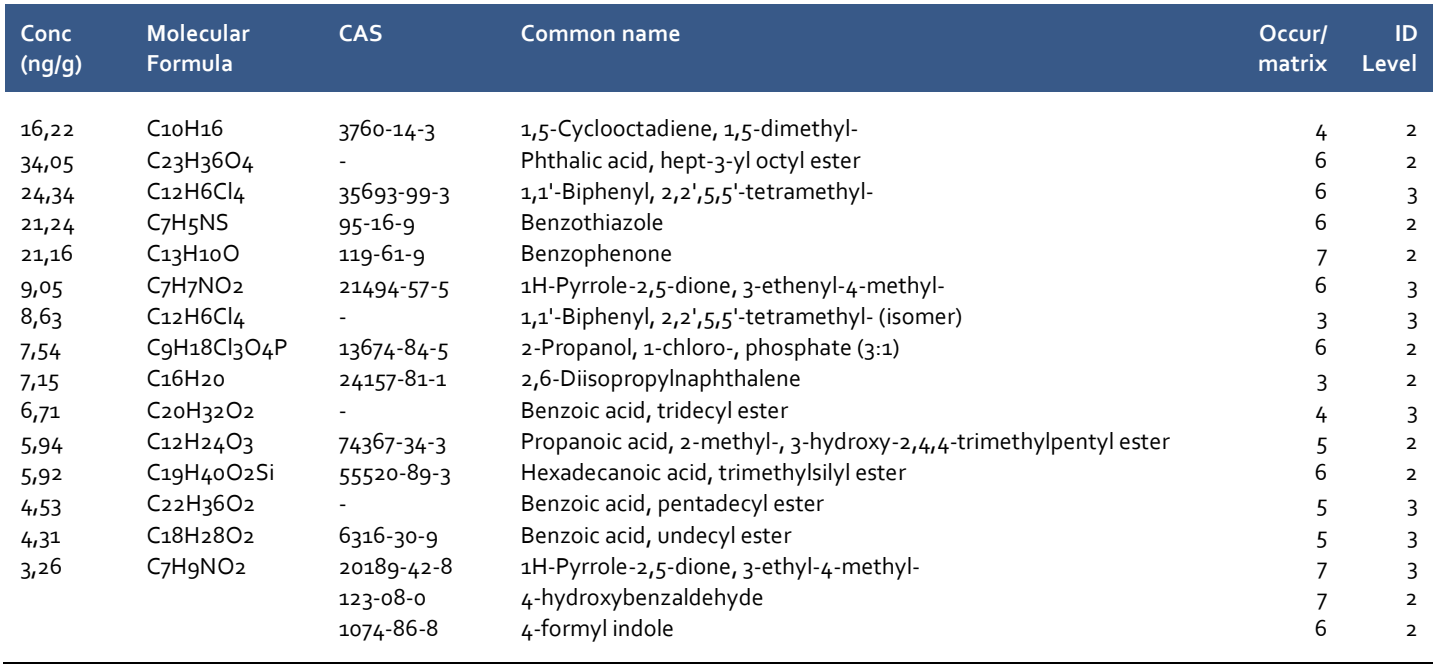


Table 24: Sediment Faroe Islands

\begin{tabular}{|c|c|c|c|c|c|}
\hline $\begin{array}{l}\text { Conc } \\
\text { (ng/g) }\end{array}$ & $\begin{array}{l}\text { Molecular } \\
\text { Formula }\end{array}$ & CAS & Common name & $\begin{array}{l}\text { Occur/ } \\
\text { matrix }\end{array}$ & $\begin{array}{r}\text { ID } \\
\text { Level }\end{array}$ \\
\hline 182,68 & $\mathrm{C}_{23} \mathrm{H}_{3} 6 \mathrm{O}_{4}$ & - & Phthalic acid, hept-3-yl octyl ester & 6 & 2 \\
\hline 56,76 & $\mathrm{C}_{2} \mathrm{OH}_{12}$ & $205-99-2$ & Benzo[b]fluoranthene & 4 & 2 \\
\hline 43,37 & $\mathrm{C}_{13} \mathrm{H}_{10} \mathrm{O}$ & $119-61-9$ & Benzophenone & 7 & 2 \\
\hline 39,51 & $\mathrm{C}_{14} \mathrm{H}_{14} \mathrm{O}$ & $19578-70-2$ & Benzene, 1-methyl-2-(phenylmethoxy)- & 5 & 2 \\
\hline 37,21 & $\mathrm{C}_{22} \mathrm{H}_{12}$ & $193-39-5$ & Indeno[1,2,3-cd]pyrene & 4 & 2 \\
\hline 32,49 & $\mathrm{C}_{12} \mathrm{H}_{6} \mathrm{Cl}_{4}$ & $35693-99-3$ & 1,1'-Biphenyl, 2,2',5,5'-tetramethyl- & 6 & 3 \\
\hline 30,45 & $\mathrm{C}_{7} \mathrm{H}_{5} \mathrm{NS}$ & $95-16-9$ & Benzothiazole & 6 & 2 \\
\hline 25,63 & $\mathrm{C}_{2} \mathrm{OH}_{12}$ & $192-97-2$ & Benzo[e]pyrene & 5 & 2 \\
\hline 25,32 & $\mathrm{C}_{2} \mathrm{OH}_{12}$ & $50-32-8$ & Benzo[a]pyrene & 5 & 2 \\
\hline 22,65 & $\mathrm{C}_{1} 6 \mathrm{H}_{10}$ & $206-44-0$ & Fluoranthene & 6 & 2 \\
\hline 16,94 & $\mathrm{C}_{18} \mathrm{H}_{12}$ & $217-59-4$ & Triphenylene & 6 & 2 \\
\hline 16,18 & $\mathrm{C}_{1} 6 \mathrm{H}_{2} \mathrm{O}$ & $24157-81-1$ & 2,6-Diisopropylnaphthalene & 3 & 2 \\
\hline 15,47 & $\mathrm{C}_{22} \mathrm{H}_{12}$ & $193-43-1$ & Indeno $[1,2,3$-cd $]$ fluoranthene & 4 & 2 \\
\hline 14,82 & $\mathrm{C}_{1} 8 \mathrm{H}_{3} \mathrm{O}$ & $2719-62-2$ & Benzene, (1-pentylheptyl)- & 4 & 2 \\
\hline 14,45 & $\mathrm{C}_{14} \mathrm{H}_{10}$ & $85-01-8$ & Phenanthrene & 6 & 2 \\
\hline 13,22 & $\mathrm{C}_{1} 8 \mathrm{H}_{18}$ & $66552-97-4$ & 2-Isopropyl-10-methylphenanthrene & 5 & 3 \\
\hline 12,45 & $\mathrm{C}_{1} 6 \mathrm{H}_{10}$ & $129-00-0$ & Pyrene & 6 & 2 \\
\hline 10,29 & $\mathrm{C}_{1} 6 \mathrm{H}_{16}$ & $7694-30-6$ & Benzene, $1,1^{\prime}-(1,2$-cyclobutanediyl)bis-, cis- & 5 & 2 \\
\hline 9,54 & $\mathrm{C}_{19} \mathrm{H}_{14}$ & $82979-72-4$ & 8,9-Dihydro-7H-cyclopenta[a]pyrene & 5 & 3 \\
\hline 8,81 & $\mathrm{C}_{10} \mathrm{H}_{16}$ & $3760-14-3$ & 1,5-Cyclooctadiene, 1,5-dimethyl- & 4 & 3 \\
\hline 8,71 & $\mathrm{C}_{9} \mathrm{H}_{1} 8 \mathrm{Cl}_{3} \mathrm{O}_{4} \mathrm{P}$ & $13674-84-5$ & 2-Propanol, 1-chloro-, phosphate (3:1) & 6 & 2 \\
\hline 8,40 & $\mathrm{C}_{2} \mathrm{OH}_{16}$ & $35187-19-0$ & 4,12-Dimethylbenz[a]anthracene & 4 & 3 \\
\hline 8,32 & $\mathrm{C}_{15} \mathrm{H}_{24}$ & $242794-76-9$ & Bicyclo[5.2.0]nonane, 2-methylene-4,8,8-trimethyl-4-vinyl- & 3 & 2 \\
\hline 8,18 & $\mathrm{C}_{12} \mathrm{H}_{24} \mathrm{O}_{3}$ & $74367-34-3$ & Propanoic acid, 2-methyl-, 3-hydroxy-2,4,4-trimethylpentyl ester & 5 & 2 \\
\hline 8,00 & $\mathrm{C}_{14} \mathrm{H}_{10}$ & $120-12-7$ & Anthracene & 6 & 2 \\
\hline 7,41 & $\mathrm{C}_{9} \mathrm{H}_{14} \mathrm{O} 6$ & $102-76-1$ & Triacetin & 3 & 2 \\
\hline 7,30 & $\mathrm{C}_{3} \mathrm{OH}_{5} \mathrm{O}$ & $1615-91-4$ & Diploptene & 4 & 3 \\
\hline 7,19 & $\mathrm{C}_{18} 8 \mathrm{H}_{12}$ & $56-55-3$ & Benz[a]anthracene & 6 & 2 \\
\hline 7,07 & $\mathrm{C}_{15} \mathrm{H}_{2} 8$ & $54832-83-6$ & $1 \mathrm{H}$-Indene, octahydro-2,2,4,4,7,7-hexamethyl-, trans- & 3 & 3 \\
\hline 6,55 & $\mathrm{C} 10 \mathrm{H} 8$ & $91-20-3$ & Naphthalene & 4 & 2 \\
\hline 6,44 & $\mathrm{C}_{9} \mathrm{H}_{12} \mathrm{O}_{2}$ & $1125-21-9$ & 2,6,6-Trimethyl-2-cyclohexene-1,4-dione & 6 & 2 \\
\hline 6,38 & $\mathrm{C}_{18} \mathrm{H}_{12}$ & $195-19-7$ & Benzo[c]phenanthrene & 5 & 2 \\
\hline 6,15 & $\mathrm{C}_{17} \mathrm{H}_{16}$ & $3674-73-5$ & Phenanthrene, 2,3,5-trimethyl- & 4 & 2 \\
\hline 6,11 & $\mathrm{C}_{1} 8 \mathrm{H}_{2} 6 \mathrm{O}$ & $1222-05-5$ & Galaxolide & 2 & 2 \\
\hline 5,78 & $\mathrm{C}_{17} \mathrm{H}_{28}$ & $4537-15-9$ & Benzene, (1-butylheptyl)- & 3 & 2 \\
\hline 5,49 & $\mathrm{C}_{2} \mathrm{OH}_{12}$ & $198-55-0$ & Perylene & 5 & 2 \\
\hline 5,44 & $\mathrm{C}_{8} \mathrm{H}_{10 O}$ & $122-99-6$ & Ethanol, 2-phenoxy- & 3 & 2 \\
\hline 5,30 & $\mathrm{C}_{17} \mathrm{H}_{12}$ & $199-94-0$ & 7H-Benzo[de]anthracene & 4 & 3 \\
\hline 5,12 & $\mathrm{C}_{1} 8 \mathrm{H}_{14} \mathrm{~S}$ & $24964-12-3$ & Benzo[b]naphtho[2,3-d]thiophene, 7,8-dimethyl- & 3 & 3 \\
\hline 4,95 & $\mathrm{C}_{15} \mathrm{H}_{12}$ & $27410-55-5$ & 8,9-Dihydrocyclopenta[def]phenanthrene & 5 & 3 \\
\hline 4,54 & $\mathrm{C}_{15} \mathrm{H}_{10}$ & $83469-43-6$ & $6 \mathrm{H}$-Cyclobuta[jk]phenanthrene & 5 & 2 \\
\hline 3,99 & $\mathrm{C}_{16} 6 \mathrm{H}_{14}$ & $3674-69-9$ & Phenanthrene, 4,5-dimethyl- & 5 & 2 \\
\hline 3,96 & $\mathrm{C}_{2} \mathrm{OH}_{12}$ & $205-82-3$ & Benzo[j]fluoranthene & 4 & 2 \\
\hline 3,51 & $\mathrm{C}_{21} \mathrm{H}_{3} 6$ & $641-85-0$ & Allopregnane & 3 & 3 \\
\hline 3,46 & $\mathrm{C}_{17} \mathrm{H}_{12}$ & $243-17-4$ & $11 \mathrm{H}$-Benzo[b]fluorene & 5 & 2 \\
\hline 3,44 & $\mathrm{C}_{10 \mathrm{H}_{14}}$ & $527-53-7$ & Benzene, 1,2,3,5-tetramethyl- & 2 & 2 \\
\hline 3,35 & $\mathrm{C}_{12} \mathrm{H} 8 \mathrm{O}$ & $132-64-9$ & Dibenzofuran & 5 & 2 \\
\hline 3,20 & $\mathrm{C}_{7} \mathrm{H}_{6} \mathrm{O}_{2}$ & $65-85-0$ & Benzoic acid & 1 & 2 \\
\hline 3,16 & $\mathrm{C}_{15} \mathrm{H}_{12}$ & $832-69-9$ & Phenanthrene, 1-methyl- & 3 & 2 \\
\hline 2,90 & $\mathrm{C}_{12} \mathrm{H}_{12}$ & - & Naphthalene, dimethyl- & 10 & 3 \\
\hline 2,87 & $\mathrm{C}_{1} 8 \mathrm{H}_{22}$ & $6566-19-4$ & 10,18-Bisnorabieta-5,7,9(10),11,13-pentaene & 3 & 3 \\
\hline 2,74 & $\mathrm{C}_{1} 8 \mathrm{H}_{10} \mathrm{O}$ & $191-37-7$ & Naphtho[2,1,8,7-klmn]xanthene & 4 & 3 \\
\hline 2,71 & $\mathrm{C}_{11} \mathrm{H}_{10}$ & $91-57-6$ & Naphthalene, 2-methyl- & 6 & 2 \\
\hline 2,69 & $\mathrm{C}_{11} \mathrm{H}_{16} 6 \mathrm{O}_{2}$ & $15356-74-8$ & $2(4 \mathrm{H})$-Benzofuranone, 5,6,7,7a-tetrahydro-4,4,7a-trimethyl- & 5 & 2 \\
\hline 2,56 & $\mathrm{C}_{21} \mathrm{H}_{14}$ & $63104-32-5$ & 10-Methylbenzo(a)pyrene & 4 & 3 \\
\hline 2,56 & $\mathrm{C}_{21} \mathrm{H}_{14}$ & $239-85-0$ & 13H-Dibenzo[a,h]fluorene & 5 & 3 \\
\hline 2,55 & $\mathrm{C}_{2} \mathrm{OH}_{32} \mathrm{O}_{2}$ & - & Benzoic acid, tridecyl ester & 4 & 3 \\
\hline 2,47 & $\mathrm{C}_{12} \mathrm{H}_{12}$ & - & Naphthalene, dimethyl- & 10 & 3 \\
\hline 2,44 & $\mathrm{C}_{11} \mathrm{H}_{10}$ & $90-12-0$ & Naphthalene, 1-methyl- & 6 & 2 \\
\hline 2,24 & $\mathrm{C}_{8} \mathrm{H}_{5} \mathrm{Br} 2 \mathrm{~N}$ & - & 2-Bromo-6-(3-bromo-prop-1-ynyl)-pyridine & 2 & 3 \\
\hline 2,15 & $\mathrm{C}_{15} \mathrm{H}_{22} \mathrm{O}_{3}$ & - & Salicylic acid, butyl ether, butyl ester & 3 & 2 \\
\hline 2,15 & $\mathrm{C}_{22} \mathrm{H}_{3} 6 \mathrm{O}_{2}$ & - & Benzoic acid, pentadecyl ester & 5 & 3 \\
\hline 2,05 & $\mathrm{C}_{1} 6 \mathrm{H}_{10 S}$ & $205-43-6$ & Benzo[b]naphtho[1,2-d]thiophene & 5 & 2 \\
\hline
\end{tabular}


Continued

\begin{tabular}{|c|c|c|c|c|c|}
\hline $\begin{array}{l}\text { Conc } \\
\text { (ng/g) }\end{array}$ & $\begin{array}{l}\text { Molecular } \\
\text { Formula }\end{array}$ & CAS & Common name & $\begin{array}{l}\text { Occur/ } \\
\text { matrix }\end{array}$ & $\begin{array}{r}\text { ID } \\
\text { Level }\end{array}$ \\
\hline 2,05 & $\mathrm{C}_{7} \mathrm{H}_{9} \mathrm{NO}_{2}$ & $20189-42-8$ & 1H-Pyrrole-2,5-dione, 3-ethyl-4-methyl- & 7 & 3 \\
\hline 2,02 & $\mathrm{C}_{1} 8 \mathrm{H}_{2} 8 \mathrm{O}_{2}$ & $6316-30-9$ & Benzoic acid, undecyl ester & 5 & ? \\
\hline 2,02 & $\mathrm{C}_{2} 4 \mathrm{H}_{3} 8 \mathrm{O}_{4}$ & $6422-86-2$ & 1,4-Benzenedicarboxylic acid, bis(2-ethylhexyl) ester & 2 & J \\
\hline 2,01 & $\mathrm{C}_{17} \mathrm{H}_{12}$ & - & 7H-Benzo[c]fluorene (isomer) & 13 & 3 \\
\hline 2,00 & $\mathrm{C}_{1} 6 \mathrm{H}_{14}$ & $1576-67-6$ & Phenanthrene, 3,6-dimethyl- & 5 & 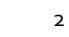 \\
\hline 1,90 & $\mathrm{C}_{13} \mathrm{H} 8 \mathrm{O}$ & $548-39-0$ & $1 \mathrm{H}$-Phenalen-1-one & 4 & . \\
\hline 1,90 & $\mathrm{C}_{17} \mathrm{H}_{12} \mathrm{~S}$ & - & 2-Methylbenzo[b]naphtho[2,1-d]thiophene & 3 & 3 \\
\hline 1,90 & $\mathrm{C}_{15} \mathrm{H}_{12}$ & $832-71-3$ & Phenanthrene, 3-methyl- & 4 & 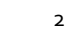 \\
\hline 1,81 & $\mathrm{C}_{13} \mathrm{H}_{14}$ & $829-26-5$ & Naphthalene, 2,3,6-trimethyl- & 4 & 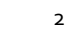 \\
\hline 1,73 & $\mathrm{C}_{1} 6 \mathrm{H}_{10 O}$ & $205-39-0$ & Benzo(b)naphtho(1,2-d)furan & 5 & 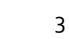 \\
\hline 1,68 & $\mathrm{C}_{1} 8 \mathrm{H}_{10}$ & $203-12-3$ & Benzo[ghi]fluoranthene & 3 & 2 \\
\hline 1,60 & $\mathrm{C}_{10 \mathrm{H}_{14}}$ & $488-23-3$ & Benzene, 1,2,3,4-tetramethyl- & 3 & 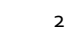 \\
\hline 1,55 & $\mathrm{C}_{11} \mathrm{H}_{20} \mathrm{O}$ & $2958-76-1$ & Naphthalene, decahydro-2-methyl- & 2 & 2 \\
\hline 1,54 & $\mathrm{C}_{21} \mathrm{H}_{14}$ & $241-28-1$ & $8 \mathrm{H}$-Indeno[2,1-b]phenanthrene & 4 & . \\
\hline 1,46 & $\mathrm{C}_{15} \mathrm{H}_{22}$ & $483-77-2$ & Naphthalene, 1,2,3,4-tetrahydro-1,6-dimethyl-4-(1-methylethyl)-, (1S-cis)- & 3 & 2 \\
\hline 1,45 & $\mathrm{C}_{13} \mathrm{H}_{10}$ & $86-73-7$ & Fluorene & 4 & 2 \\
\hline 1,40 & $\mathrm{C}_{17} \mathrm{H}_{12}$ & - & $7 \mathrm{H}$-Benzo[c]fluorene (isomer) & 13 & 3 \\
\hline 1,39 & $\mathrm{C}_{19} \mathrm{H}_{4} \mathrm{OO} 2 \mathrm{Si}$ & $55520-89-3$ & Hexadecanoic acid, trimethylsilyl ester & 6 & 2 \\
\hline 1,38 & $\mathrm{C}_{19} \mathrm{H}_{12}$ & $50861-05-7$ & 9H-Cyclopenta[a]pyrene & 5 & 2 \\
\hline 1,32 & $\mathrm{C}_{13} \mathrm{H}_{10 O}$ & $3218-36-8$ & {$\left[1,1^{\prime}\right.$-Biphenyl]-4-carboxaldehyde } & 4 & 2 \\
\hline 1,27 & $\mathrm{C}_{1} 6 \mathrm{H}_{12}$ & $6232-48-0$ & Acephenanthrylene, 4,5-dihydro- & 5 & 2 \\
\hline 1,23 & $\mathrm{C}_{13} \mathrm{H}_{10 O}$ & $92-83-1$ & $9 \mathrm{H}-$ Xanthene & 5 & 2 \\
\hline 1,17 & $\mathrm{C}_{6} \mathrm{H}_{5} \mathrm{BrO}$ & $591-20-8$ & Phenol, 3-bromo- & 2 & 2 \\
\hline 1,15 & $\mathrm{C}_{15} \mathrm{H}_{12}$ & $832-64-4$ & Phenanthrene, 4-methyl- & 4 & 2 \\
\hline 1,12 & $\mathrm{C}_{14} \mathrm{H}_{14}$ & $613-33-2$ & 4,4'-Dimethylbiphenyl & 3 & 2 \\
\hline 1,10 & $\mathrm{C}_{1} 6 \mathrm{H}_{11} \mathrm{~N}$ & - & $11 \mathrm{H}$-Benzo[a]carbazole (isomer) & 7 & 3 \\
\hline 1,02 & $\mathrm{C}_{8} \mathrm{H}_{8} \mathrm{O}_{2}$ & $582-24-1$ & Ethanone, 2-hydroxy-1-phenyl- & 3 & 2 \\
\hline 1,02 & $\mathrm{C}_{1} 6 \mathrm{H}_{10 O}$ & $243-42-5$ & Benzo[b]naphtho[2,3-d]furan & 4 & 3 \\
\hline 0,95 & $\mathrm{C}_{14} \mathrm{H}_{12} \mathrm{~S}$ & $1136-85-2$ & 3,7-Dimethyldibenzothiophene & 3 & 2 \\
\hline 0,90 & $\mathrm{C}_{14} \mathrm{H}_{8} \mathrm{O}_{2}$ & $84-65-1$ & 9,10 -Anthracenedione & 5 & 2 \\
\hline 0,90 & $\mathrm{C}_{12} \mathrm{H}_{5} \mathrm{Cl}_{5}$ & $32598-14-4$ & 1,1'-Biphenyl, 2,3,3',4,4'-pentachloro- & 2 & 2 \\
\hline 0,84 & $\mathrm{C}_{17} \mathrm{H}_{12}$ & - & $7 \mathrm{H}$-Benzo[c]fluorene (isomer) & 13 & - \\
\hline 0,79 & $\mathrm{C}_{12} \mathrm{H}_{12} \mathrm{O}_{5}$ & $5062-30-6$ & Phenacylidene diacetate & 2 & 2 \\
\hline 0,77 & $\mathrm{C}_{7} \mathrm{H}_{7} \mathrm{NO}_{2}$ & $21494-57-5$ & $1 \mathrm{H}$-Pyrrole-2,5-dione, 3-ethenyl-4-methyl- & 6 & - \\
\hline 0,77 & $\mathrm{C}_{11} \mathrm{H}_{18} \mathrm{O}_{4}$ & - & Fumaric acid, ethyl 3-methylbut-2-yl ester & 3 & 2 \\
\hline 0,74 & $\mathrm{C}_{16} \mathrm{H}_{11} \mathrm{~N}$ & - & $11 \mathrm{H}-B e n z o[a] c a r b a z o l e(i s o m e r)$ & 7 & 3 \\
\hline 0,63 & $\mathrm{C}_{15} \mathrm{H} 8 \mathrm{O}$ & $5737-13-3$ & Cyclopenta(def)phenanthrenone & 4 & 2 \\
\hline 0,62 & $\mathrm{C}_{21} \mathrm{H}_{44} \mathrm{O}_{2} \mathrm{Si}$ & $18748-91-9$ & Octadecanoic acid, trimethylsilyl ester & 1 & 2 \\
\hline 0,56 & $\mathrm{C}_{12} \mathrm{H}_{5} \mathrm{Cl}_{5}$ & $55215-17-3$ & 1,1'-Biphenyl, 2,2',3,4,6-Pentachloro- & 1 & 2 \\
\hline \multirow[t]{12}{*}{0,54} & $\mathrm{C}_{13} \mathrm{H}_{12}$ & $643-93-6$ & 1,1'-Biphenyl, 3-methyl- & 3 & 2 \\
\hline & $\mathrm{C}_{15} \mathrm{H}_{14} \mathrm{~N}_{4} \mathrm{O}_{2} \mathrm{~S}$ & $526-08-9$ & sulfaphenazole & 1 & 2 \\
\hline & $\mathrm{C}_{5} \mathrm{H}_{10} \mathrm{O}_{4} \mathrm{~S}$ & $30618-84-9$ & mercaptoacetic acid, monoester with propane-1,2,3-triol & & \\
\hline & $\mathrm{C}_{4} \mathrm{H}_{10 \mathrm{O}_{3} \mathrm{~S}}$ & $2386-47-2$ & 1-butan-sulfonic acid & 1 & 2 \\
\hline & $\mathrm{C}_{7} \mathrm{H}_{6} \mathrm{O}_{2}$ & $100-83-4$ & 3-hydroxybenzaldehyde & 1 & 2 \\
\hline & $\mathrm{C}_{7} \mathrm{H}_{6} \mathrm{O}_{2}$ & $65-85-0$ & Benzoic acid & 2 & 2 \\
\hline & $\mathrm{C}_{9} \mathrm{H}_{14} \mathrm{O}_{4}$ & $86273-46-3$ & 2-(2-Vinyloxyethoxy)ethyl acrylate & 1 & 2 \\
\hline & $\mathrm{C}_{1} 6 \mathrm{H}_{10} \mathrm{ClN}_{3} \mathrm{O}_{3}$ & $2814-77-9$ & 1-[(2-chloro-4-nitrophenyl)azo]-2-naphthol & 1 & 2 \\
\hline & & $5835-26-7$ & isopimaric acid & 4 & 2 \\
\hline & & $123-08-0$ & 4-hydroxybenzaldehyde & 7 & 2 \\
\hline & & $1074-86-8$ & 4-formyl indole & 6 & 2 \\
\hline & & $92612-52-7$ & PFHXA & 4 & 2 \\
\hline
\end{tabular}


Table 25: Fish Finland

\begin{tabular}{|c|c|c|c|c|c|}
\hline $\begin{array}{l}\text { Conc } \\
\text { (ng/g) }\end{array}$ & $\begin{array}{l}\text { Molecular } \\
\text { Formula }\end{array}$ & CAS & Common name & $\begin{array}{l}\text { Occur/ } \\
\text { matrix }\end{array}$ & $\begin{array}{r}\text { ID } \\
\text { Level }\end{array}$ \\
\hline 1673,34 & $\mathrm{C}_{14} \mathrm{H}_{2} 6 \mathrm{O}_{2}$ & $544-64-9$ & Myristoleic acid & 6 & 2 \\
\hline 600,59 & $\mathrm{C}_{17} \mathrm{H}_{3} 6 \mathrm{O}_{2} \mathrm{Si}$ & $18603-17-3$ & Tetradecanoic acid, trimethylsilyl ester & 7 & 2 \\
\hline 588,59 & $\mathrm{C}_{8} \mathrm{H}_{12} \mathrm{O}$ & $30086-02-3$ & 3,5-Octadien-2-one, $(E, E)$ - & 7 & 2 \\
\hline 484,62 & $\mathrm{C}_{1} 9 \mathrm{H}_{4} 0 \mathrm{O}_{2} \mathrm{Si}$ & $55520-89-3$ & Hexadecanoic acid, trimethylsilyl ester & 7 & 2 \\
\hline 472,13 & $\mathrm{C}_{2} 4 \mathrm{H}_{3} 8 \mathrm{O}_{4}$ & - & Phthalic acid, di(6-methylhept-2-yl) ester & 2 & 2 \\
\hline 313,62 & $\mathrm{C}_{23} \mathrm{H}_{4} \mathrm{OO}_{3}$ & $71041-49-1$ & 11-(3,4-Dimethyl-5-pentyl-2-furyl)-dodecanoic acid, methyl ester & 6 & 2 \\
\hline 235,49 & $\mathrm{C}_{17} \mathrm{H}_{34} \mathrm{O}$ & - & Hexadecanal, methyl- & 2 & 2 \\
\hline 209,20 & $\mathrm{C}_{1} 8 \mathrm{H}_{3} 8 \mathrm{O}_{2} \mathrm{Si}$ & $74367-22-9$ & n-Pentadecanoic acid, trimethylsilyl ester & 6 & 2 \\
\hline 181,78 & $\mathrm{C}_{17} \mathrm{H}_{25} \mathrm{NO}_{4}$ & - & Succinic acid, 2-(dimethylamino)ethyl 4-isopropylphenyl ester & 5 & 2 \\
\hline 180,42 & $\mathrm{C}_{7} \mathrm{H}_{5} \mathrm{NS}$ & $95-16-9$ & Benzothiazole & 5 & 2 \\
\hline 108,22 & $\mathrm{C}_{11} \mathrm{H}_{18} \mathrm{O}_{4}$ & - & Fumaric acid, ethyl 3-methylbut-2-yl ester & 7 & 2 \\
\hline 105,74 & $\mathrm{C}_{7} \mathrm{H}_{6} \mathrm{O}_{2}$ & $65-85-0$ & Benzoic acid & 7 & 2 \\
\hline 101,96 & $\mathrm{C}_{9} \mathrm{H}_{12} \mathrm{O}_{2}$ & $1125-21-9$ & 2,6,6-Trimethyl-2-cyclohexene-1,4-dione & 4 & 2 \\
\hline 97,54 & $\mathrm{C}_{9} \mathrm{H}_{10} \mathrm{O}_{3}$ & $10139-84-1$ & 2',4'-Dihydroxy-3'-methylacetophenone & 5 & 2 \\
\hline 92,87 & $\mathrm{C}_{14} \mathrm{H}_{14} \mathrm{O}$ & $19578-70-2$ & Benzene, 1-methyl-2-(phenylmethoxy)- & 3 & 2 \\
\hline 74,40 & $\mathrm{C}_{9} \mathrm{H}_{10} \mathrm{O}_{2}$ & $140-11-4$ & Acetic acid, phenylmethyl ester & 3 & 2 \\
\hline 61,54 & $\mathrm{C}_{10 \mathrm{H}_{14} \mathrm{O}_{2}}$ & $1948-33-0$ & t-Butylhydroquinone & 5 & 2 \\
\hline 60,51 & $\mathrm{C}_{21} \mathrm{H}_{3} 6 \mathrm{O}_{3}$ & $86879-46-1$ & 11-(3,4-Dimethyl-5-propyl-2-furyl)-undecanoic acid, methyl ester & 6 & 2 \\
\hline 44,09 & $\mathrm{C} 8 \mathrm{H}_{6} \mathrm{O}_{2}$ & $1074-12-0$ & Phenylglyoxal & 5 & 2 \\
\hline 31,71 & $\mathrm{C}_{12} \mathrm{H}_{24} \mathrm{O}_{3}$ & $74367-34-3$ & Propanoic acid, 2-methyl-, 3-hydroxy-2,4,4-trimethylpentyl ester & 7 & 2 \\
\hline 25,94 & $\mathrm{C}_{12} \mathrm{H}_{12} \mathrm{O}_{5}$ & $5062-30-6$ & Phenacylidene diacetate & 4 & 2 \\
\hline 23,76 & $\mathrm{C}_{14} \mathrm{H}_{14}$ & $611-43-8$ & 1,1'-Biphenyl, 2,3'-dimethyl- & 3 & 2 \\
\hline 19,30 & $\mathrm{C}_{5} \mathrm{H}_{9} \mathrm{NO}_{2}$ & $4394-85-8$ & N-Formylmorpholine & 7 & 2 \\
\hline 16,88 & $\mathrm{C} 6 \mathrm{H}_{8} \mathrm{O}_{3}$ & $29393-32-6$ & $2(3 \mathrm{H})$-Furanone, 5 -acetyldihydro- & 3 & 2 \\
\hline 15,65 & $\mathrm{C}_{12} \mathrm{H}_{6} \mathrm{Cl}_{4}$ & $35693-99-3$ & 1,1'-Biphenyl, 2,2',5,5'-tetramethyl- & 7 & 3 \\
\hline 12,51 & $\mathrm{C}_{14} \mathrm{H}_{14}$ & $611-61-0$ & 1,1'-Biphenyl, 2,4'-dimethyl- & 3 & 2 \\
\hline 8,65 & $\mathrm{C}_{14} \mathrm{H}_{14}$ & $613-33-2$ & 4,4'-Dimethylbiphenyl & 3 & 2 \\
\hline 8,26 & $\mathrm{C}_{9} \mathrm{H}_{1} 8 \mathrm{Cl}_{3} \mathrm{O}_{4} \mathrm{P}$ & $13674-84-5$ & 2-Propanol, 1-chloro-, phosphate (3:1) & 5 & 2 \\
\hline 7,88 & $\mathrm{C}_{12} \mathrm{H}_{4} \mathrm{Cl} 6$ & $74472-43-8$ & $2,3,3^{\prime}, 4,5^{\prime}, 6$-Hexachloro-1,1'-biphenyl & 4 & 2 \\
\hline 6,49 & $\mathrm{C}_{12} \mathrm{H}_{4} \mathrm{Cl} 6$ & $52663-72-6$ & 1,1'-Biphenyl, $2,3^{\prime}, 4,4^{\prime}, 5,5^{\prime}$-hexachloro- & 2 & 2 \\
\hline 5,77 & $\mathrm{C}_{8} \mathrm{H}_{10 O} \mathrm{O}_{2}$ & $122-99-6$ & Ethanol, 2-phenoxy- & 1 & 2 \\
\hline 4,91 & $\mathrm{C}_{2} \mathrm{OH}_{32} \mathrm{O}_{2}$ & - & Benzoic acid, tridecyl ester & 1 & 3 \\
\hline 4,83 & $\mathrm{C}_{1} 6 \mathrm{H}_{2} \mathrm{O}$ & $24157-81-1$ & 2,6-Diisopropylnaphthalene & 2 & 2 \\
\hline 4,73 & $\mathrm{C}_{12} \mathrm{H}_{5} \mathrm{Cl}_{5}$ & $55215-17-3$ & 1,1'-Biphenyl, 2,2',3,4,6-Pentachloro- & 3 & 2 \\
\hline 4,62 & $\mathrm{C}_{9} \mathrm{H}_{10} \mathrm{O}_{2}$ & $70-70-2$ & Paroxypropione & 5 & 2 \\
\hline 3,85 & $\mathrm{C}_{6} \mathrm{H}_{11} \mathrm{NO}_{2}$ & $1696-20-4$ & Morpholine, 4-acetyl- & 2 & 3 \\
\hline 2,66 & $\mathrm{C}_{13} \mathrm{H}_{16}$ & $30364-38-6$ & Naphthalene, 1,2-dihydro-1,1,6-trimethyl- & 3 & 2 \\
\hline 2,25 & $\mathrm{C}_{12} \mathrm{H}_{3} \mathrm{Cl}_{7}$ & $35065-30-6$ & 1,1'-Biphenyl, 2,2',3,3',4,4',5-heptachloro- & 2 & 2 \\
\hline Qualitative & $\mathrm{C}_{6} \mathrm{H}_{4} \mathrm{Br}_{2} \mathrm{O}$ & $615-58-7$ & 2,4-dibromophenol & 7 & 2 \\
\hline Qualitative & $\mathrm{C}_{7} \mathrm{H}_{5} \mathrm{Br}_{3}$ & $607-99-8$ & 2,4,6-tribromoanisole & 7 & 2 \\
\hline Qualitative & $\mathrm{C}_{6} \mathrm{H}_{3} \mathrm{Br}_{3} \mathrm{O}$ & $118-79-6$ & $2,4,6$-Tribromophenol & 7 & 2 \\
\hline Qualitative & $\mathrm{C}_{12} \mathrm{H}_{5} \mathrm{Cl}_{5}$ & $31508-00-6$ & PCB-118 & 7 & 2 \\
\hline Qualitative & $\mathrm{C}_{12} \mathrm{H}_{4} \mathrm{Cl} 6$ & $35065-27-1$ & PCB-153 & 7 & 2 \\
\hline Qualitative & $\mathrm{C}_{12} \mathrm{H}_{5} \mathrm{Cl}_{5}$ & $32598-14-4$ & PCB-105 & 5 & 2 \\
\hline Qualitative & $\mathrm{C}_{12} \mathrm{H}_{4} \mathrm{Cl} 6$ & $35065-28-2$ & PCB-138 & 7 & 2 \\
\hline Qualitative & $\mathrm{C}_{12} \mathrm{H}_{4} \mathrm{Cl} 6$ & $52663-72-6$ & PCB-167 & 7 & 2 \\
\hline Qualitative & $\mathrm{C}_{12} \mathrm{H}_{4} \mathrm{Cl} 6$ & $38380-08-4$ & PCB-156 & 7 & 2 \\
\hline Qualitative & $\mathrm{C}_{12} \mathrm{H}_{4} \mathrm{Cl} 6$ & $69782-90-7$ & PCB-157 & 7 & 2 \\
\hline Qualitative & $\mathrm{C}_{12} \mathrm{H}_{3} \mathrm{Cl}_{7}$ & $35065-29-3$ & PCB-180 & 7 & 2 \\
\hline Qualitative & $\mathrm{C}_{12} \mathrm{H}_{6 \mathrm{Br}} 4 \mathrm{O}$ & $189084-61-5$ & $2,3^{\prime}, 4^{\prime}, 6$-Tetrabromodiphenyl ether (PBDE\# 47) & 7 & 2 \\
\hline Qualitative & $\mathrm{C}_{12} \mathrm{H}_{3} \mathrm{Cl}_{7}$ & $35065-30-6$ & PCB-170 & 7 & 2 \\
\hline Qualitative & $\mathrm{C}_{12} \mathrm{H}_{3} \mathrm{Cl}_{7}$ & $39635-31-9$ & PCB-189 & 7 & 2 \\
\hline Qualitative & $\mathrm{C}_{12} \mathrm{H}_{5} \mathrm{Br}_{5} \mathrm{O}$ & $93703-48-1$ & $2,2^{\prime}, 3,4,4^{\prime}$-Pentabromodiphenyl ether (PBDE\#100) & 7 & 2 \\
\hline Qualitative & $\mathrm{C}_{12} \mathrm{H}_{2} \mathrm{Cl} 8$ & $52663-78-2$ & PCB-195 & 7 & 3 \\
\hline Qualitative & $\mathrm{C}_{12} \mathrm{H}_{2} \mathrm{Cl} 8$ & $35694-08-7$ & PCB-194 & 7 & 2 \\
\hline Qualitative & $\mathrm{C}_{12} \mathrm{H}_{5} \mathrm{Br}_{5} \mathrm{O}$ & $60348-60-9$ & $2,2^{\prime}, 4,4^{\prime}, 6$-Pentabromodiphenyl ether (PBDE\#99) & 7 & 2 \\
\hline Qualitative & $\mathrm{C}_{12} \mathrm{HCl}_{9}$ & 40186-72-9 & PCB-206 & 7 & 2 \\
\hline Qualitative & $\mathrm{C}_{12} \mathrm{Cl}_{10}$ & $2051-24-3$ & PCB-209 & 7 & 2 \\
\hline Qualitative & $\mathrm{C}_{12} \mathrm{H}_{4} \mathrm{Br} 6 \mathrm{O}$ & $68631-49-2$ & $2,2^{\prime}, 4,4^{\prime}, 5,5^{\prime}$-Hexabromodiphenyl ether (PBDE\#154) & 7 & 2 \\
\hline Qualitative & $\mathrm{C}_{15} \mathrm{H}_{12} \mathrm{Br}_{4} \mathrm{O}_{2}$ & $79-94-7$ & Tetrabromobisphenol A (TBBP-A) & 7 & 2 \\
\hline Qualitative & $\mathrm{C}_{9} \mathrm{H}_{10} \mathrm{O}_{3}$ & $6161-65-5$ & 2-methoxy-6-methylbenzoic acid & 1 & 3 \\
\hline Qualitative & $\mathrm{C}_{1} \mathrm{OH}_{1} 6 \mathrm{~N}_{2} \mathrm{O}_{4}$ & $10543-57-4$ & $\mathrm{~N}, \mathrm{~N}^{\prime}$-ethylenebis $[\mathrm{N}$-acetylacetamide $]$ & 1 & 3 \\
\hline Qualitative & $\mathrm{C}_{5} \mathrm{H}_{4} \mathrm{~N}_{4} \mathrm{O}_{2}$ & $2465-59-0$ & oxypurinol & 1 & 3 \\
\hline
\end{tabular}


Continued

\begin{tabular}{|c|c|c|c|c|c|}
\hline $\begin{array}{l}\text { Conc } \\
\text { (ng/g) }\end{array}$ & $\begin{array}{l}\text { Molecular } \\
\text { Formula }\end{array}$ & CAS & Common name & $\begin{array}{l}\text { Occur/ } \\
\text { matrix }\end{array}$ & $\begin{array}{r}\text { ID } \\
\text { Level }\end{array}$ \\
\hline Qualitative & $\mathrm{C}_{8} \mathrm{H}_{11} \mathrm{NO}_{3}$ & $65-23-6$ & pyridoxine & 1 & 3 \\
\hline Qualitative & $\mathrm{C}_{1} \mathrm{OH}_{13} \mathrm{~N}_{5} \mathrm{O}_{5}$ & $118-00-3$ & Guanosine & 1 & 3 \\
\hline Qualitative & $\mathrm{C}_{1} \mathrm{OH}_{12} \mathrm{~N}_{4} \mathrm{O}_{5}$ & $16220-07-8$ & Allopurinol riboside & 2 & 3 \\
\hline Qualitative & $\mathrm{C}_{12} \mathrm{H}_{15} \mathrm{~N}_{5} \mathrm{O}_{5}$ & $75128-73-3$ & 2-[(2-acetamido-6,9-dihydro-6-oxo-1H-purin-9-yl]methoxy]ethyl acetate & 2 & 3 \\
\hline Qualitative & $\mathrm{C}_{11} \mathrm{H}_{2} \mathrm{ON}_{2} \mathrm{O}_{5}$ & $27317-69-7$ & $\mathrm{~N}$-[N-[(1,1-dimethylethoxy)carbonyl]-L-alanyl]-L-alanine & 1 & 3 \\
\hline Qualitative & $\mathrm{C}_{1} 6 \mathrm{H}_{32} \mathrm{O}_{2}$ & $57-10-3$ & Hexadecanoic acid & 1 & 3 \\
\hline Qualitative & $\mathrm{C}_{1} 6 \mathrm{H}_{10} \mathrm{ClN} \mathrm{O}_{3}$ & $2814-77-9$ & 1-[(2-chloro-4-nitrophenyl)azo]-2-naphthol & 1 & 3 \\
\hline Qualitative & $\mathrm{C}_{22} \mathrm{H}_{32} \mathrm{O}_{2}$ & $127-47-9$ & retinyl acetate & 1 & 3 \\
\hline Qualitative & $\mathrm{C}_{8} \mathrm{HF}_{17} \mathrm{O}_{3} \mathrm{~S}$ & $1763-23-1$ & Perfluorooctane sulfonate (PFOS) (Acid) & 1 & 3 \\
\hline Qualitative & $\mathrm{C}_{5} \mathrm{H}_{4} \mathrm{~N}_{4} \mathrm{O}$ & $315-30-0$ & allopurinol & 1 & 3 \\
\hline Qualitative & $\mathrm{C}_{8} \mathrm{H}_{1} 8 \mathrm{~N}_{2} \mathrm{O}_{4} \mathrm{~S}$ & $7365-45-9$ & 4-(2-hydroxyethyl)piperazin-1-ylethanesulphonic acid & 1 & 3 \\
\hline Qualitative & $\mathrm{C}_{1} 8 \mathrm{H}_{3} 6 \mathrm{O}_{2}$ & $57-11-4$ & Octadecanoic acid & 1 & 3 \\
\hline Qualitative & $\mathrm{C}_{19} \mathrm{H}_{3} 8 \mathrm{O}_{2}$ & $68517-10-2$ & methyl isostearate & 1 & 3 \\
\hline Qualitative & $\mathrm{C}_{19} \mathrm{H}_{3} 8 \mathrm{O}_{2}$ & $142-91-6$ & Hexadecanoic acid, 1-methylethyl ester & 1 & 3 \\
\hline Qualitative & $\mathrm{C}_{19} \mathrm{H}_{3} 8 \mathrm{O}_{2}$ & $112-61-8$ & methyl stearate & 1 & 3 \\
\hline Qualitative & $\mathrm{C}_{8} \mathrm{H}_{21} \mathrm{NOSi}_{2}$ & $10416-59-8$ & trimethylsilyl $\mathrm{N}$-trimethylsilylacetamidate & 1 & 3 \\
\hline Qualitative & $\mathrm{C}_{6} \mathrm{H}_{9} \mathrm{NO}_{2}$ & $72291-30-6$ & Propanoic acid, 2-cyano-2-methyl-, methyl ester & 1 & 3 \\
\hline Qualitative & $\mathrm{C}_{11} \mathrm{H}_{12} \mathrm{~N}_{2} \mathrm{O}_{2}$ & $73-22-3$ & Tryptophan & 1 & 3 \\
\hline Qualitative & $\mathrm{C}_{9} \mathrm{H}_{14} \mathrm{O}_{3}$ & $2455-24-5$ & tetrahydrofurfuryl methacrylate & 1 & 3 \\
\hline Qualitative & $\mathrm{C}_{31} \mathrm{H}_{4} 8 \mathrm{O}_{2} \mathrm{~S}_{2}$ & $23288-49-5$ & probucol & 1 & 3 \\
\hline Qualitative & $\mathrm{C}_{6} \mathrm{H}_{9} \mathrm{~N}_{3} \mathrm{O}_{2}$ & $4998-57-6$ & histidine & 1 & 3 \\
\hline Qualitative & $\mathrm{C}_{4} \mathrm{H}_{6} \mathrm{O}_{4}$ & $110-15-6$ & succinic acid & 1 & 3 \\
\hline Qualitative & $\mathrm{C}_{12} \mathrm{H}_{8} \mathrm{O}_{4}$ & $298-81-7$ & methoxsalen & 1 & 3 \\
\hline Qualitative & $\mathrm{C}_{12} \mathrm{H}_{2} \mathrm{~N}_{2} \mathrm{O}_{3}$ & $6425-39-4$ & 2,2'-dimorpholinyldiethyl ether & 1 & 3 \\
\hline Qualitative & $\mathrm{C}_{2} 6 \mathrm{H}_{45} \mathrm{NO}_{7} \mathrm{~S}$ & $81-24-3$ & taurocholic acid & 2 & 3 \\
\hline Qualitative & $\mathrm{C}_{5} \mathrm{H}_{9} \mathrm{NO}_{2}$ & $923-02-4$ & $\mathrm{~N}$-(hydroxymethyl)methacrylamide & 1 & 3 \\
\hline Qualitative & $\mathrm{C}_{5} \mathrm{H}_{9} \mathrm{NO}_{2}$ & $7646-67-5$ & $\mathrm{~N}$-(2-hydroxyethyl)prop-2-enamide & 1 & 3 \\
\hline Qualitative & $\mathrm{C}_{5} \mathrm{H}_{9} \mathrm{NO}_{2}$ & $81778-07-6$ & 4,4-dimethyl-1,2-oxazolidin-3-one & 1 & 3 \\
\hline \multirow[t]{11}{*}{ Qualitative } & $\mathrm{C}_{5} \mathrm{H}_{9} \mathrm{NO}_{2}$ & $14205-39-1$ & methyl 3-aminocrotonate & 1 & 3 \\
\hline & & 474511-07-4 & PFNS & 2 & 2 \\
\hline & & $171978-95-3$ & PFDoDA & 2 & 2 \\
\hline & & $39108-34-4$ & $8: 2$ FTS & 1 & 2 \\
\hline & & $355-46-4$ & $\mathrm{PFH} \times \mathrm{S}$ & 2 & 2 \\
\hline & & $106-44-5$ & $\mathrm{p}$-cresol & 2 & 3 \\
\hline & & $550-24-3$ & embelin & 2 & 3 \\
\hline & & $352-21-6$ & aminohydroxybutyric acid & 2 & 3 \\
\hline & & $123-08-0$ & 4-hydroxybenzaldehyde & 6 & 3 \\
\hline & & $1074-86-8$ & 4 -formyl indole & 6 & 3 \\
\hline & & $66621-73-6$ & 2-pyrimidine acetic acid & 3 & 3 \\
\hline
\end{tabular}


Table 26: Fish Sweden

\begin{tabular}{|c|c|c|c|c|c|}
\hline $\begin{array}{l}\text { Conc } \\
\text { (ng/g) }\end{array}$ & $\begin{array}{l}\text { Molecular } \\
\text { Formula }\end{array}$ & CAS & Common name & $\begin{array}{l}\text { Occur/ } \\
\text { matrix }\end{array}$ & $\begin{array}{r}\text { ID } \\
\text { Level }\end{array}$ \\
\hline 3342,56 & $\mathrm{C}_{14} \mathrm{H}_{2} 6 \mathrm{O}_{2}$ & $544-64-9$ & Myristoleic acid & 6 & 2 \\
\hline 1717,17 & $\mathrm{C}_{17} \mathrm{H}_{25} \mathrm{NO}_{4}$ & - & Succinic acid, 2-(dimethylamino)ethyl 4-isopropylphenyl ester & 5 & 2 \\
\hline 888,20 & $\mathrm{C}_{17} \mathrm{H}_{3} 6 \mathrm{O}_{2} \mathrm{Si}$ & $18603-17-3$ & Tetradecanoic acid, trimethylsilyl ester & 7 & 2 \\
\hline 696,89 & $\mathrm{C}_{8} \mathrm{H}_{12} \mathrm{O}$ & $30086-02-3$ & 3,5-Octadien-2-one, $(E, E)$ - & 7 & 2 \\
\hline 588,78 & $\mathrm{C}_{23} \mathrm{H}_{4} \mathrm{OO}_{3}$ & 71041-49-1 & 11-(3,4-Dimethyl-5-pentyl-2-furyl)-dodecanoic acid, methyl ester & 6 & 2 \\
\hline 318,22 & $\mathrm{C}_{19} \mathrm{H}_{4} \mathrm{OO} 2 \mathrm{Si}$ & $55520-89-3$ & Hexadecanoic acid, trimethylsilyl ester & 7 & 2 \\
\hline 249,74 & $\mathrm{C}_{7} \mathrm{H}_{5} \mathrm{NS}$ & $95-16-9$ & Benzothiazole & 5 & 2 \\
\hline 249,70 & $\mathrm{C}_{23} \mathrm{H}_{3} 6 \mathrm{O}_{4}$ & - & Phthalic acid, hept-3-yl octyl ester & 2 & 2 \\
\hline 207,32 & $\mathrm{C}_{9} \mathrm{H}_{10} \mathrm{O}_{3}$ & $10139-84-1$ & 2',4'-Dihydroxy-3'-methylacetophenone & 5 & 2 \\
\hline 205,96 & $\mathrm{C}_{9} \mathrm{H}_{12} \mathrm{O}_{2}$ & $1125-21-9$ & 2,6,6-Trimethyl-2-cyclohexene-1,4-dione & 4 & 2 \\
\hline 148,81 & $\mathrm{C}_{11} \mathrm{H}_{18} 8 \mathrm{O}_{4}$ & - & Fumaric acid, ethyl 3-methylbut-2-yl ester & 7 & 2 \\
\hline 147,46 & $\mathrm{C}_{9} \mathrm{H}_{10 O}$ & $122-46-3$ & m-Cresyl acetate & 1 & 2 \\
\hline 146,65 & $\mathrm{C}_{7} \mathrm{H}_{6} \mathrm{O}_{2}$ & $65-85-0$ & Benzoic acid & 7 & 2 \\
\hline 124,83 & $\mathrm{C}_{14} \mathrm{H}_{14} \mathrm{O}$ & $19578-70-2$ & Benzene, 1-methyl-2-(phenylmethoxy)- & 3 & 2 \\
\hline 104,67 & $\mathrm{C}_{21} \mathrm{H}_{3} 6 \mathrm{O}_{3}$ & $86879-46-1$ & 11-(3,4-Dimethyl-5-propyl-2-furyl)-undecanoic acid, methyl ester & 6 & 2 \\
\hline 62,20 & $\mathrm{C}_{10 \mathrm{H}_{14} \mathrm{O}_{2}}$ & $1948-33-0$ & t-Butylhydroquinone & 5 & 2 \\
\hline 55,45 & $\mathrm{C} 6 \mathrm{H}_{8} \mathrm{O}_{3}$ & $29393-32-6$ & $2\left({ }_{3} \mathrm{H}\right)$-Furanone, 5 -acetyldihydro- & 3 & 2 \\
\hline 48,03 & $\mathrm{Cg} \mathrm{H} 8 \mathrm{O}$ & $83-33-0$ & $1 \mathrm{H}$-Inden-1-one, 2,3-dihydro- & 2 & 2 \\
\hline 42,96 & $\mathrm{C}_{1} 8 \mathrm{H}_{2} 6 \mathrm{O}$ & $1222-05-5$ & Galaxolide & 4 & 2 \\
\hline 42,72 & $\mathrm{C}_{14} \mathrm{H}_{14}$ & $611-43-8$ & 1,1'-Biphenyl, 2,3'-dimethyl- & 3 & 2 \\
\hline 36,38 & $\mathrm{C}_{12} \mathrm{H}_{12} \mathrm{O}_{5}$ & $5062-30-6$ & Phenacylidene diacetate & 4 & 2 \\
\hline 26,79 & $\mathrm{C}_{8} \mathrm{H}_{16} 6 \mathrm{O}$ & $5715-23-1$ & 3,4-Dimethylcyclohexanol & 6 & 2 \\
\hline 26,21 & $\mathrm{C}_{8} \mathrm{H}_{7} \mathrm{NS}_{2}$ & $615-22-5$ & Benzothiazole, 2-(methylthio)- & 3 & 2 \\
\hline 23,19 & $\mathrm{C}_{12} \mathrm{H}_{24} \mathrm{O}_{3}$ & $74367-34-3$ & Propanoic acid, 2-methyl-, 3-hydroxy-2,4,4-trimethylpentyl ester & 7 & 2 \\
\hline 22,74 & $\mathrm{C}_{9} \mathrm{H}_{10 O} \mathrm{O}_{2}$ & $70-70-2$ & Paroxypropione & 5 & 2 \\
\hline 18,94 & $\mathrm{C}_{14} \mathrm{H}_{14}$ & $611-61-0$ & 1,1'-Biphenyl, 2,4'-dimethyl- & 3 & 2 \\
\hline 18,63 & $\mathrm{C} 8 \mathrm{H}_{6} \mathrm{O}_{2}$ & $1074-12-0$ & Phenylglyoxal & 5 & 2 \\
\hline 16,57 & $\mathrm{C}_{12} \mathrm{H}_{6} \mathrm{Cl}_{4}$ & $35693-99-3$ & 1,1'-Biphenyl, 2,2',5,5'-tetramethyl- & 7 & 3 \\
\hline 15,70 & $\mathrm{C}_{5} \mathrm{H}_{9} \mathrm{NO}_{2}$ & $4394-85-8$ & N-Formylmorpholine & 7 & 2 \\
\hline 13,27 & $\mathrm{C}_{7} \mathrm{H}_{5} \mathrm{NOS}$ & $934-34-9$ & $2(3 \mathrm{H})$-Benzothiazolone & 3 & 3 \\
\hline 12,48 & $\mathrm{C}_{7} \mathrm{H}_{8} \mathrm{O}_{2}$ & $504-15-4$ & Orcinol & 2 & 2 \\
\hline 11,66 & $\mathrm{C}_{14} \mathrm{H}_{14}$ & $613-33-2$ & 4,4'-Dimethylbiphenyl & 3 & 2 \\
\hline 8,19 & $\mathrm{C} 8 \mathrm{H}_{8} \mathrm{O}_{2}$ & $582-24-1$ & Ethanone, 2-hydroxy-1-phenyl- & 1 & 2 \\
\hline 7,47 & $\mathrm{C}_{9} \mathrm{H}_{8} \mathrm{O}_{3}$ & $7310-95-4$ & 2-Hydroxy-5-methylisophthalaldehyde & 1 & 2 \\
\hline 7,30 & $\mathrm{C}_{12} \mathrm{H}_{5} \mathrm{Cl}_{5}$ & $55215-17-3$ & 1,1'-Biphenyl, 2,2',3,4,6-Pentachloro- & 3 & 2 \\
\hline 6,18 & $\mathrm{C}_{13} \mathrm{H}_{16}$ & $30364-38-6$ & Naphthalene, 1,2-dihydro-1,1,6-trimethyl- & 3 & 2 \\
\hline 5,93 & $\mathrm{C}_{12} \mathrm{H}_{4} \mathrm{Cl} 6$ & $74472-43-8$ & $2,3,3^{\prime}, 4,5^{\prime}, 6$-Hexachloro-1, 1'-biphenyl & 4 & 2 \\
\hline 5,44 & $\mathrm{C}_{9} \mathrm{H}_{1} 8 \mathrm{Cl}_{3} \mathrm{O}_{4} \mathrm{P}$ & $13674-84-5$ & 2-Propanol, 1-chloro-, phosphate (3:1) & 5 & 2 \\
\hline 3,45 & $\mathrm{C}_{17} \mathrm{H}_{34} \mathrm{O}$ & 55019-46-0 & Hexadecanal, 2-methyl- & 3 & 2 \\
\hline 1,14 & $\mathrm{C}_{17} \mathrm{H}_{34} \mathrm{O}$ & - & Hexadecanal, methyl- & 2 & 2 \\
\hline Qualitative & $\mathrm{C}_{6} \mathrm{H}_{4} \mathrm{Br}_{2} \mathrm{O}$ & $615-58-7$ & 2,4-dibromophenol & 7 & 2 \\
\hline Qualitative & $\mathrm{C}_{7} \mathrm{H}_{5} \mathrm{Br}_{3}$ & $607-99-8$ & 2,4,6-tribromoanisole & 7 & 2 \\
\hline Qualitative & $\mathrm{C}_{6} \mathrm{H}_{3} \mathrm{Br}_{3} \mathrm{O}$ & $118-79-6$ & $2,4,6$-Tribromophenol & 7 & 2 \\
\hline Qualitative & $\mathrm{C}_{12} \mathrm{H}_{5} \mathrm{Cl}_{5}$ & $31508-00-6$ & PCB-118 & 7 & 2 \\
\hline Qualitative & $\mathrm{C}_{12} \mathrm{H}_{4} \mathrm{Cl} 6$ & $35065-27-1$ & PCB-153 & 7 & 2 \\
\hline Qualitative & $\mathrm{C}_{12} \mathrm{H}_{5} \mathrm{Cl}_{5}$ & $32598-14-4$ & PCB-105 & 5 & 2 \\
\hline Qualitative & $\mathrm{C}_{12} \mathrm{H}_{4} \mathrm{Cl} 6$ & $35065-28-2$ & PCB-138 & 7 & 2 \\
\hline Qualitative & $\mathrm{C}_{12} \mathrm{H}_{4} \mathrm{Cl} 6$ & $52663-72-6$ & PCB-167 & 7 & 2 \\
\hline Qualitative & $\mathrm{C}_{12} \mathrm{H}_{4} \mathrm{Cl} 6$ & $38380-08-4$ & PCB-156 & 7 & 2 \\
\hline Qualitative & $\mathrm{C}_{12} \mathrm{H}_{4} \mathrm{Cl} 6$ & $69782-90-7$ & PCB-157 & 7 & 2 \\
\hline Qualitative & $\mathrm{C}_{12} \mathrm{H}_{3} \mathrm{Cl}_{7}$ & $35065-29-3$ & PCB-180 & 7 & 2 \\
\hline Qualitative & $\mathrm{C}_{12} \mathrm{H}_{6} \mathrm{Br}_{4} \mathrm{O}$ & $189084-61-5$ & $2,3^{\prime}, 4^{\prime}, 6$-Tetrabromodiphenyl ether (PBDE\#47) & 7 & 2 \\
\hline Qualitative & $\mathrm{C}_{12} \mathrm{H}_{3} \mathrm{Cl}_{7}$ & $35065-30-6$ & PCB-170 & 7 & 2 \\
\hline Qualitative & $\mathrm{C}_{12} \mathrm{H}_{3} \mathrm{Cl}_{7}$ & $39635-31-9$ & PCB-189 & 7 & 2 \\
\hline Qualitative & $\mathrm{C}_{12} \mathrm{H}_{5} \mathrm{Br}_{5} \mathrm{O}$ & $93703-48-1$ & $2,2^{\prime}, 3,4,4^{\prime}$-Pentabromodiphenyl ether (PBDE\#100) & 7 & 2 \\
\hline Qualitative & $\mathrm{C}_{12} \mathrm{H}_{2} \mathrm{Cl} 8$ & $52663-78-2$ & PCB-195 & 7 & 3 \\
\hline Qualitative & $\mathrm{C}_{12} \mathrm{H}_{2} \mathrm{Cl} 8$ & $35694-08-7$ & PCB-194 & 7 & 2 \\
\hline Qualitative & $\mathrm{C}_{12} \mathrm{H}_{5} \mathrm{Br}_{5} \mathrm{O}$ & $60348-60-9$ & $2,2^{\prime}, 4,4^{\prime}, 6$-Pentabromodiphenyl ether (PBDE\#99) & 7 & 2 \\
\hline Qualitative & $\mathrm{C}_{12} \mathrm{HCl} 9$ & 40186-72-9 & PCB-206 & 7 & 2 \\
\hline Qualitative & $\mathrm{C}_{12} \mathrm{Cl}_{10}$ & $2051-24-3$ & PCB-209 & 7 & 2 \\
\hline Qualitative & $\mathrm{C}_{12} \mathrm{H}_{4} \mathrm{Br} 6 \mathrm{O}$ & $68631-49-2$ & $2,2^{\prime}, 4,4^{\prime}, 5,5^{\prime}$-Hexabromodiphenyl ether (PBDE\#154) & 7 & 2 \\
\hline Qualitative & $\mathrm{C}_{15} \mathrm{H}_{12} \mathrm{Br}_{4} \mathrm{O}_{2}$ & $79-94-7$ & Tetrabromobisphenol A (TBBP-A) & 7 & 2 \\
\hline Qualitative & $\mathrm{C}_{23} \mathrm{H}_{32} \mathrm{~N}_{2} \mathrm{O}_{4}$ & $243973-20-8$ & pinoxaden & 1 & 3 \\
\hline
\end{tabular}


Continued

\begin{tabular}{|c|c|c|c|c|c|}
\hline $\begin{array}{l}\text { Conc } \\
\text { (ng/g) }\end{array}$ & $\begin{array}{l}\text { Molecular } \\
\text { Formula }\end{array}$ & CAS & Common name & $\begin{array}{c}\text { Occur/ } \\
\text { matrix }\end{array}$ & $\begin{array}{r}\text { ID } \\
\text { Level }\end{array}$ \\
\hline Qualitative & $\mathrm{C}_{24} \mathrm{H}_{34} \mathrm{O}_{4}$ & $82543-17-7$ & & 1 & 3 \\
\hline Qualitative & $\mathrm{C}_{9} \mathrm{H}_{17} \mathrm{NO}_{3} \mathrm{~S}$ & $35130-97-3$ & Tropine-3-mesylate & 1 & 3 \\
\hline Qualitative & $\mathrm{C}_{15} \mathrm{H}_{12} \mathrm{~N}_{2} \mathrm{O}_{2}$ & $36507-30-9$ & 10,11-Epoxycarbamazepine & 1 & 3 \\
\hline Qualitative & $\mathrm{C}_{2} 5 \mathrm{H}_{3} \mathrm{NO}_{4}$ & $89365-50-4$ & & 1 & 3 \\
\hline Qualitative & $\mathrm{C}_{23} \mathrm{H}_{15} \mathrm{~N}_{3} \mathrm{O}$ & $380917-97-5$ & & 1 & 3 \\
\hline \multirow[t]{11}{*}{ Qualitative } & $\mathrm{C}_{2} \mathrm{OH}_{32} \mathrm{O}_{2}$ & $34434-80-5$ & & 2 & 3 \\
\hline & & 474511-07-4 & PFNS & 2 & 2 \\
\hline & $\mathrm{C}_{12} \mathrm{HF}_{23} \mathrm{O}_{2}$ & $171978-95-3$ & PFDoDA & 2 & 2 \\
\hline & & $80705-13-1$ & 4:3 Acid & 1 & 2 \\
\hline & & $355-46-4$ & PFHxS & 2 & 2 \\
\hline & & $106-44-5$ & p-cresol & 2 & 3 \\
\hline & & $352-21-6$ & aminohydroxybutyric acid & 2 & 3 \\
\hline & & $123-08-0$ & 4-hydroxybenzaldehyde & 6 & 3 \\
\hline & & $1074-86-8$ & 4-formyl indole & 6 & 3 \\
\hline & & $60-32-2$ & aminocaproic acid & 4 & 3 \\
\hline & & $66621-73-6$ & 2-pyrimidine acetic acid & 3 & 3 \\
\hline
\end{tabular}


Table 27: Fish Denmark

\begin{tabular}{|c|c|c|c|c|c|}
\hline Conc (ng/g) & $\begin{array}{l}\text { Molecular } \\
\text { Formula }\end{array}$ & CAS & Common name & $\begin{array}{l}\text { Occur/ } \\
\text { matrix }\end{array}$ & $\begin{array}{r}\text { ID } \\
\text { Level }\end{array}$ \\
\hline 3070,12 & $\mathrm{C}_{14} \mathrm{H}_{2} 6 \mathrm{O}_{2}$ & $544-64-9$ & Myristoleic acid & 6 & 2 \\
\hline 1055,79 & $\mathrm{C}_{23} \mathrm{H}_{4} \mathrm{OO}_{3}$ & 71041-49-1 & 11-(3,4-Dimethyl-5-pentyl-2-furyl)-dodecanoic acid, methyl ester & 6 & 2 \\
\hline 698,51 & $\mathrm{C}_{8} \mathrm{H}_{12} \mathrm{O}$ & $30086-02-3$ & 3,5-Octadien-2-one, $(\mathrm{E}, \mathrm{E})$ - & 7 & 2 \\
\hline 615,66 & $\mathrm{C}_{21} \mathrm{H}_{3} 6 \mathrm{O}_{3}$ & $86879-46-1$ & 11-(3,4-Dimethyl-5-propyl-2-furyl)-undecanoic acid, methyl ester & 6 & 2 \\
\hline 481,65 & $\mathrm{C}_{17} \mathrm{H}_{3} 6 \mathrm{O}_{2} \mathrm{Si}$ & $18603-17-3$ & Tetradecanoic acid, trimethylsilyl ester & 7 & 2 \\
\hline 454,13 & $\mathrm{C}_{17} \mathrm{H}_{25} \mathrm{NO}_{4}$ & - & Succinic acid, 2-(dimethylamino)ethyl 4-isopropylphenyl ester & 5 & 2 \\
\hline 426,38 & $\mathrm{C}_{7} \mathrm{H}_{5} \mathrm{NS}$ & $95-16-9$ & Benzothiazole & 5 & 2 \\
\hline 318,84 & $\mathrm{C}_{2} 4 \mathrm{H}_{3} 8 \mathrm{O}_{4}$ & - & Phthalic acid, di(2-propylpentyl) ester & 3 & 2 \\
\hline 267,72 & $\mathrm{C}_{1} 9 \mathrm{H}_{4} 0 \mathrm{O}_{2} \mathrm{Si}$ & $55520-89-3$ & Hexadecanoic acid, trimethylsilyl ester & 7 & 2 \\
\hline 241,15 & $\mathrm{C}_{11} \mathrm{H}_{18} 8 \mathrm{O}_{4}$ & - & Fumaric acid, ethyl 3-methylbut-2-yl ester & 7 & 2 \\
\hline 193,72 & $\mathrm{C}_{13} \mathrm{H}_{20} \mathrm{O}$ & $14901-07-6$ & 3-Buten-2-one, 4-(2,6,6-trimethyl-1-cyclohexen-1-yl)- & 4 & 2 \\
\hline 192,92 & $\mathrm{C}_{8} \mathrm{H}_{16} 6 \mathrm{O}$ & $5715-23-1$ & 3,4-Dimethylcyclohexanol & 6 & 2 \\
\hline 181,47 & $\mathrm{C}_{1} 8 \mathrm{H}_{3} 8 \mathrm{O}_{2} \mathrm{Si}$ & $74367-22-9$ & n-Pentadecanoic acid, trimethylsilyl ester & 6 & 2 \\
\hline 113,86 & $\mathrm{C}_{1} 6 \mathrm{H}_{33} \mathrm{NO}$ & $629-54-9$ & Hexadecanamide & 4 & 2 \\
\hline 87,73 & $\mathrm{C}_{10 \mathrm{H}_{14} \mathrm{O}_{2}}$ & $1948-33-0$ & t-Butylhydroquinone & 5 & 2 \\
\hline 75,30 & $\mathrm{C}_{9} \mathrm{H}_{10} \mathrm{O}_{2}$ & $140-11-4$ & Acetic acid, phenylmethyl ester & 3 & 2 \\
\hline 57,57 & $\mathrm{C}_{12} \mathrm{H}_{12} \mathrm{O}_{5}$ & $5062-30-6$ & Phenacylidene diacetate & 4 & 2 \\
\hline 51,26 & $\mathrm{C}_{7} \mathrm{H}_{5} \mathrm{NOS}$ & $934-34-9$ & $2(3 \mathrm{H})$-Benzothiazolone & 3 & 3 \\
\hline 50,73 & $\mathrm{C}_{11} \mathrm{H}_{16 \mathrm{O}_{2}}$ & $17092-92-1$ & $2(4 \mathrm{H})$-Benzofuranone, 5,6,7,7a-tetrahydro-4,4,7a-trimethyl-, (R)- & 3 & 2 \\
\hline 44,01 & $\mathrm{C}_{7} \mathrm{H}_{6} \mathrm{O}_{2}$ & $65-85-0$ & Benzoic acid & 7 & 2 \\
\hline 43,64 & $\mathrm{C}_{9} \mathrm{H}_{10} \mathrm{O}_{3}$ & $10139-84-1$ & $2^{\prime}, 4^{\prime}-$ Dihydroxy-3'-methylacetophenone & 5 & 2 \\
\hline 42,39 & $\mathrm{C}_{9} \mathrm{H}_{10 O} \mathrm{O}_{2}$ & $70-70-2$ & Paroxypropione & 5 & 2 \\
\hline 41,05 & $\mathrm{Cg} \mathrm{H} 8 \mathrm{O}$ & $83-33-0$ & $1 \mathrm{H}$-Inden-1-one, 2,3-dihydro- & 2 & 2 \\
\hline 39,21 & $\mathrm{C}_{12} \mathrm{H}_{24} \mathrm{O}_{3}$ & $74367-34-3$ & Propanoic acid, 2-methyl-, 3-hydroxy-2,4,4-trimethylpentyl ester & 7 & 2 \\
\hline 37,95 & $\mathrm{C}_{14} \mathrm{H}_{14}$ & $611-43-8$ & 1,1'-Biphenyl, 2,3'-dimethyl- & 3 & 2 \\
\hline 37,70 & $\mathrm{C}_{7} \mathrm{H}_{8} \mathrm{O}_{2}$ & $504-15-4$ & Orcinol & 2 & 2 \\
\hline 31,78 & $\mathrm{C}_{8} \mathrm{H}_{7} \mathrm{NS}_{2}$ & $615-22-5$ & Benzothiazole, 2-(methylthio)- & 3 & 2 \\
\hline 29,24 & $\mathrm{C}_{14} \mathrm{H}_{8} \mathrm{~N}_{2} \mathrm{~S}_{2}$ & $4271-09-4$ & 2,2'-Bibenzothiazole & 1 & 3 \\
\hline 28,86 & $\mathrm{C}_{12} \mathrm{H}_{6} \mathrm{Cl}_{4}$ & $35693-99-3$ & 1,1'-Biphenyl, 2,2',5,5'-tetramethyl- & 7 & 3 \\
\hline 26,56 & $\mathrm{C} 8 \mathrm{H}_{6} \mathrm{O}_{2}$ & $1074-12-0$ & Phenylglyoxal & 5 & 2 \\
\hline 16,96 & $\mathrm{C}_{14} \mathrm{H}_{14}$ & $611-61-0$ & 1,1'-Biphenyl, 2,4'-dimethyl- & 3 & 2 \\
\hline 14,70 & $\mathrm{C}_{5} \mathrm{H}_{9} \mathrm{NO}_{2}$ & $4394-85-8$ & N-Formylmorpholine & 7 & 2 \\
\hline 13,57 & $\mathrm{C}_{9} \mathrm{H}_{12} \mathrm{O}_{2}$ & $1125-21-9$ & 2,6,6-Trimethyl-2-cyclohexene-1,4-dione & 4 & 2 \\
\hline 10,53 & $\mathrm{C}_{14} \mathrm{H}_{14}$ & $613-33-2$ & 4,4'-Dimethylbiphenyl & 3 & 2 \\
\hline 10,12 & $\mathrm{C} 6 \mathrm{H}_{8} \mathrm{O}_{3}$ & $29393-32-6$ & $2(3 \mathrm{H})$-Furanone, 5 -acetyldihydro- & 3 & 2 \\
\hline 9,73 & $\mathrm{C}_{7} \mathrm{H}_{7} \mathrm{NO}_{2}$ & $21494-57-5$ & 1H-Pyrrole-2,5-dione, 3-ethenyl-4-methyl- & 1 & 3 \\
\hline 6,25 & $\mathrm{C}_{6} \mathrm{H}_{11} \mathrm{NO}_{2}$ & $1696-20-4$ & Morpholine, 4-acetyl- & 2 & 3 \\
\hline 5,41 & $\mathrm{C}_{1} \mathrm{OH}_{21} \mathrm{NO}$ & $1118-92-9$ & Octanamide, N,N-dimethyl- & 2 & 2 \\
\hline 4,13 & $\mathrm{C}_{9} \mathrm{H}_{18} \mathrm{Cl}_{3} \mathrm{O}_{4} \mathrm{P}$ & $13674-84-5$ & 2-Propanol, 1-chloro-, phosphate (3:1) & 5 & 2 \\
\hline 4,09 & $\mathrm{C}_{10 \mathrm{H}}$ & $91-20-3$ & Naphthalene & 1 & 2 \\
\hline 3,90 & $\mathrm{C}_{15} \mathrm{H}_{22} \mathrm{O}_{3}$ & - & Salicylic acid, butyl ether, butyl ester & 5 & 2 \\
\hline 3,59 & $\mathrm{C}_{10 \mathrm{H}_{22} \mathrm{O}_{3}}$ & 29911-28-2 & 2-Propanol, 1-(2-butoxy-1-methylethoxy)- & 1 & 3 \\
\hline 3,33 & $\mathrm{Cg} \mathrm{H} 8 \mathrm{O}$ & $4432-63-7$ & 2-Phenylpropenal & 4 & 2 \\
\hline 3,26 & $\mathrm{C}_{11} \mathrm{H}_{10}$ & $91-57-6$ & Naphthalene, 2-methyl- & 1 & 2 \\
\hline Qualitative & $\mathrm{C}_{6} \mathrm{H}_{4} \mathrm{Br}_{2} \mathrm{O}$ & $615-58-7$ & 2,4-dibromophenol & 7 & 2 \\
\hline Qualitative & $\mathrm{C}_{7} \mathrm{H}_{5} \mathrm{Br}_{3}$ & $607-99-8$ & 2,4,6-tribromoanisole & 7 & 2 \\
\hline Qualitative & $\mathrm{C}_{6} \mathrm{H}_{3} \mathrm{Br}_{3} \mathrm{O}$ & $118-79-6$ & $2,4,6$-Tribromophenol & 7 & 2 \\
\hline Qualitative & $\mathrm{C}_{12} \mathrm{H}_{5} \mathrm{Cl}_{5}$ & $31508-00-6$ & PCB-118 & 7 & 2 \\
\hline Qualitative & $\mathrm{C}_{12} \mathrm{H}_{4} \mathrm{Cl} 6$ & $35065-27-1$ & PCB-153 & 7 & 2 \\
\hline Qualitative & $\mathrm{C}_{12} \mathrm{H}_{5} \mathrm{Cl}_{5}$ & $32598-14-4$ & PCB-105 & 5 & 2 \\
\hline Qualitative & $\mathrm{C}_{12} \mathrm{H}_{4} \mathrm{Cl} 6$ & $35065-28-2$ & PCB-138 & 7 & 2 \\
\hline Qualitative & $\mathrm{C}_{12} \mathrm{H}_{4} \mathrm{Cl} 6$ & $52663-72-6$ & PCB-167 & 7 & 2 \\
\hline Qualitative & $\mathrm{C}_{12} \mathrm{H}_{4} \mathrm{Cl} 6$ & $38380-08-4$ & PCB-156 & 7 & 2 \\
\hline Qualitative & $\mathrm{C}_{12} \mathrm{H}_{4} \mathrm{Cl} 6$ & $69782-90-7$ & PCB-157 & 7 & 2 \\
\hline Qualitative & $\mathrm{C}_{12} \mathrm{H}_{3} \mathrm{Cl}_{7}$ & $35065-29-3$ & PCB-18o & 7 & 2 \\
\hline Qualitative & $\mathrm{C}_{12} \mathrm{H}_{6} \mathrm{Br}_{4} \mathrm{O}$ & $189084-61-5$ & $2,3^{\prime}, 4^{\prime}, 6$-Tetrabromodiphenyl ether (PBDE\# 47) & 7 & 2 \\
\hline Qualitative & $\mathrm{C}_{12} \mathrm{H}_{3} \mathrm{Cl}_{7}$ & $35065-30-6$ & PCB-170 & 7 & 2 \\
\hline Qualitative & $\mathrm{C}_{12} \mathrm{H}_{3} \mathrm{Cl}_{7}$ & $39635-31-9$ & PCB-189 & 7 & 2 \\
\hline Qualitative & $\mathrm{C}_{12} \mathrm{H}_{5} \mathrm{Br}_{5} \mathrm{O}$ & $93703-48-1$ & $2,2^{\prime}, 3,4,4^{\prime}$-Pentabromodiphenyl ether (PBDE\#100) & 7 & 2 \\
\hline Qualitative & $\mathrm{C}_{12} \mathrm{H}_{2} \mathrm{Cl} 8$ & $52663-78-2$ & PCB-195 & 7 & 3 \\
\hline Qualitative & $\mathrm{C}_{12} \mathrm{H}_{2} \mathrm{Cl} 8$ & $35694-08-7$ & PCB-194 & 7 & 2 \\
\hline Qualitative & $\mathrm{C}_{12} \mathrm{H}_{5} \mathrm{Br}_{5} \mathrm{O}$ & $60348-60-9$ & $2,2^{\prime}, 4,4^{\prime}, 6$-Pentabromodiphenyl ether (PBDE\#99) & 7 & 2 \\
\hline Qualitative & $\mathrm{C}_{12} \mathrm{HCl}_{9}$ & 40186-72-9 & PCB-206 & 7 & 2 \\
\hline
\end{tabular}


Continued

\begin{tabular}{|c|c|c|c|c|c|}
\hline Conc (ng/g) & $\begin{array}{l}\text { Molecular } \\
\text { Formula }\end{array}$ & CAS & Common name & $\begin{array}{l}\text { Occur/ } \\
\text { matrix }\end{array}$ & $\begin{array}{l}\text { ID } \\
\text { Level }\end{array}$ \\
\hline Qualitative & $\mathrm{C}_{12} \mathrm{Cl}_{10}$ & $2051-24-3$ & PCB-209 & 7 & 2 \\
\hline Qualitative & $\mathrm{C}_{12} \mathrm{H}_{4} \mathrm{Br} 6 \mathrm{O}$ & $68631-49-2$ & $2,2^{\prime}, 4,4^{\prime}, 5,5^{\prime}$-Hexabromodiphenyl ether (PBDE\#154) & 7 & 2 \\
\hline \multirow[t]{9}{*}{ Qualitative } & $\mathrm{C}_{15} \mathrm{H}_{12} \mathrm{Br}_{4} \mathrm{O}_{2}$ & $\begin{array}{l}79-94-7 \\
76811-98-8\end{array}$ & $\begin{array}{l}\text { Tetrabromobisphenol A (TBBP-A) } \\
\text { Benzeneacetic acid, 4-[4-[4-(hydroxydiphenylmethyl)-1-piperidinyl]- } \\
\text { 1-oxobutyl]- } \alpha, \alpha \text {-dimethyl- }\end{array}$ & 7 & 2 \\
\hline & & $61432-55-1$ & Dimepiperate & 1 & 3 \\
\hline & & $58-63-9$ & Inosine & 2 & 3 \\
\hline & & $16220-07-8$ & Allopurinol riboside & 2 & 3 \\
\hline & & $2387-59-9$ & carbocisteine & 1 & 3 \\
\hline & & $34306-42-8$ & (S)-2-((tert)-butoxycarbonyl)amino)butanoic acid & & \\
\hline & & $123-08-0$ & 4-hydroxybenzaldehyde & 6 & 3 \\
\hline & & $1074-86-8$ & 4-formyl indole & 6 & 3 \\
\hline & & $66621-73-6$ & 2-pyrimidine acetic acid & 3 & 3 \\
\hline
\end{tabular}


Table 28: Fish Iceland

\begin{tabular}{|c|c|c|c|c|c|}
\hline Conc (ng/g) & $\begin{array}{l}\text { Molecular } \\
\text { Formula }\end{array}$ & CAS & Common name & $\begin{array}{l}\text { Occur/ } \\
\text { matrix }\end{array}$ & $\begin{array}{r}\text { ID } \\
\text { Level }\end{array}$ \\
\hline 17849,44 & $\mathrm{C}_{12} \mathrm{H}_{22}$ & $1008-80-6$ & Naphthalene, decahydro-2,3-dimethyl- & 2 & 3 \\
\hline 7225,30 & $\mathrm{C}_{23} \mathrm{H}_{4} \mathrm{OO}_{3}$ & 71041-49-1 & 11-(3,4-Dimethyl-5-pentyl-2-furyl)-dodecanoic acid, methyl ester & 6 & 2 \\
\hline 3814,04 & $\mathrm{C}_{11} \mathrm{H}_{20}$ & $2958-76-1$ & Naphthalene, decahydro-2-methyl- & 2 & 2 \\
\hline 3708,65 & $\mathrm{C}_{14} \mathrm{H}_{2} 6 \mathrm{O}_{2}$ & $544-64-9$ & Myristoleic acid & 6 & 2 \\
\hline 2687,54 & $\mathrm{C}_{2} 4 \mathrm{H}_{3} 8 \mathrm{O}_{4}$ & - & Phthalic acid, di(2-propylpentyl) ester & 3 & 2 \\
\hline 1889,93 & $\mathrm{C}_{17} \mathrm{H}_{3} 6 \mathrm{O}_{2} \mathrm{Si}$ & $18603-17-3$ & Tetradecanoic acid, trimethylsilyl ester & 7 & 2 \\
\hline 1593,13 & $\mathrm{C}_{11} \mathrm{H}_{20}$ & - & trans-Decalin, 2-methyl- & 2 & 2 \\
\hline 1520,04 & $\mathrm{C}_{21} \mathrm{H}_{3} 6 \mathrm{O}_{3}$ & $86879-46-1$ & 11-(3,4-Dimethyl-5-propyl-2-furyl)-undecanoic acid, methyl ester & 6 & 2 \\
\hline 1378,49 & $\mathrm{C}_{1} 8 \mathrm{H}_{3} 8 \mathrm{O}_{2} \mathrm{Si}$ & $74367-22-9$ & n-Pentadecanoic acid, trimethylsilyl ester & 6 & 2 \\
\hline 1264,38 & $\mathrm{C}_{17} \mathrm{H}_{28}$ & $4537-15-9$ & Benzene, (1-butylheptyl)- & 2 & 2 \\
\hline 1038,66 & $\mathrm{C}_{12} \mathrm{H}_{22}$ & $66660-41-1$ & 2-Ethyldecahydronaphthalene, $(Z, E)$ - & 2 & 2 \\
\hline 862,66 & $\mathrm{C}_{19} \mathrm{H}_{4} \mathrm{OO}_{2} \mathrm{Si}$ & $55520-89-3$ & Hexadecanoic acid, trimethylsilyl ester & 7 & 2 \\
\hline 800,29 & $\mathrm{C}_{1} 8 \mathrm{H}_{3} \mathrm{O}$ & $2719-62-2$ & Benzene, (1-pentylheptyl)- & 2 & 2 \\
\hline 458,16 & $\mathrm{C}_{1} 6 \mathrm{H}_{33} \mathrm{NO}$ & $629-54-9$ & Hexadecanamide & 4 & 2 \\
\hline 457,56 & $\mathrm{C}_{1} 8 \mathrm{H}_{37} \mathrm{NO}$ & - & Octadecanamide (branched) & 1 & 3 \\
\hline 308,52 & $\mathrm{C}_{15} \mathrm{H}_{28}$ & $54832-83-6$ & $1 \mathrm{H}$-Indene, octahydro- $2,2,4,4,7,7$-hexamethyl-, trans- & 2 & 2 \\
\hline 246,38 & $\mathrm{C}_{15} \mathrm{H}_{24}$ & $242794-76-9$ & Bicyclo[5.2.0]nonane, 2-methylene-4,8,8-trimethyl-4-vinyl- & 3 & 2 \\
\hline 225,80 & $\mathrm{C}_{17} \mathrm{H}_{25} \mathrm{NO}_{4}$ & - & Succinic acid, 2-(dimethylamino)ethyl 4-isopropylphenyl ester & 5 & 2 \\
\hline 99,43 & $\mathrm{C}_{13} \mathrm{H}_{2} \mathrm{OO}$ & $14901-07-6$ & 3-Buten-2-one, 4-(2,6,6-trimethyl-1-cyclohexen-1-yl)- & 4 & 2 \\
\hline 86,31 & $\mathrm{C}_{8} \mathrm{H}_{12} \mathrm{O}$ & $30086-02-3$ & 3,5-Octadien-2-one, (E,E)- & 7 & 2 \\
\hline 85,27 & $\mathrm{C}_{12} \mathrm{H}_{6} \mathrm{Cl}_{4}$ & $35693-99-3$ & 1,1'-Biphenyl, 2,2',5,5'-tetramethyl- & 7 & 3 \\
\hline 83,78 & $\mathrm{C}_{12} \mathrm{H}_{4} \mathrm{Cl} 6$ & $74472-43-8$ & $2,3,3^{\prime}, 4,5^{\prime}, 6$-Hexachloro-1,1'-biphenyl & 4 & 2 \\
\hline 78,42 & $\mathrm{C} 8 \mathrm{H}_{1} 6 \mathrm{O}$ & $5715-23-1$ & 3,4-Dimethylcyclohexanol & 6 & 2 \\
\hline 67,62 & $\mathrm{C}_{11} \mathrm{H}_{18} \mathrm{O}_{4}$ & - & Fumaric acid, ethyl 3-methylbut-2-yl ester & 7 & 2 \\
\hline 62,68 & $\mathrm{C}_{12} \mathrm{H}_{24} \mathrm{O}_{3}$ & $74367-34-3$ & Propanoic acid, 2-methyl-, 3-hydroxy-2,4,4-trimethylpentyl ester & 7 & 2 \\
\hline 60,34 & $\mathrm{C}_{1} 8 \mathrm{H}_{2} 6 \mathrm{O}$ & $1222-05-5$ & Galaxolide & 4 & 2 \\
\hline 57,73 & $\mathrm{C}_{7} \mathrm{H}_{6} \mathrm{O}_{2}$ & $65-85-0$ & Benzoic acid & 7 & 2 \\
\hline 46,51 & $\mathrm{C}_{12} \mathrm{H}_{5} \mathrm{Cl}_{5}$ & $32598-14-4$ & 1,1'-Biphenyl, 2,3,3',4,4'-pentachloro- & 2 & 2 \\
\hline 46,28 & $\mathrm{C}_{1} 6 \mathrm{H}_{2} \mathrm{O}$ & $24157-81-1$ & 2,6-Diisopropylnaphthalene & 2 & 2 \\
\hline 32,89 & $\mathrm{C}_{15} \mathrm{H}_{22}$ & $483-77-2$ & Naphthalene, 1,2,3,4-tetrahydro-1,6-dimethyl-4-(1-methylethyl)-, (1S-cis)- & 1 & 2 \\
\hline 28,34 & $\mathrm{C}_{1} \mathrm{OH} 21 \mathrm{NO}$ & $1118-92-9$ & Octanamide, N,N-dimethyl- & 2 & 2 \\
\hline 26,99 & $\mathrm{C}_{13} \mathrm{H}_{10 O}$ & $119-61-9$ & Benzophenone & 1 & 2 \\
\hline 26,69 & $\mathrm{C}_{11} \mathrm{H}_{16} 6 \mathrm{O}_{2}$ & $17092-92-1$ & $2(4 \mathrm{H})$-Benzofuranone, 5,6,7,7a-tetrahydro-4,4,7a-trimethyl-, $(\mathrm{R})$ - & 3 & 2 \\
\hline 20,12 & $\mathrm{C}_{9} \mathrm{H} 8 \mathrm{O}$ & $4432-63-7$ & 2-Phenylpropenal & 4 & 2 \\
\hline 14,40 & $\mathrm{C}_{5} \mathrm{H}_{9} \mathrm{NO}_{2}$ & $4394-85-8$ & N-Formylmorpholine & 7 & 2 \\
\hline 7,99 & $\mathrm{C}_{9} \mathrm{H}_{10 O}$ & $10139-84-1$ & 2',4'-Dihydroxy-3'-methylacetophenone & 5 & 2 \\
\hline Qualitative & $\mathrm{C}_{6} \mathrm{H}_{4} \mathrm{Br}_{2} \mathrm{O}$ & $615-58-7$ & 2,4-dibromophenol & 7 & 2 \\
\hline Qualitative & $\mathrm{C}_{7} \mathrm{H}_{5} \mathrm{Br}_{3}$ & $607-99-8$ & 2,4,6-tribromoanisole & 7 & 2 \\
\hline Qualitative & $\mathrm{C}_{6} \mathrm{H}_{3} \mathrm{Br}_{3} \mathrm{O}$ & $118-79-6$ & 2,4,6-Tribromophenol & 7 & 2 \\
\hline Qualitative & $\mathrm{C}_{12} \mathrm{H} 8 \mathrm{Br} 2 \mathrm{O}$ & $147217-71-8$ & 2,4'-Dibromodiphenyl ether (PBDE\#7) & 4 & 2 \\
\hline Qualitative & - & - & Natural product, $\mathrm{Br}_{3} \mathrm{Cl}_{3}$, probably monoterpene & 4 & 5 \\
\hline Qualitative & $\mathrm{C}_{12} \mathrm{H}_{5} \mathrm{Cl}_{5}$ & $31508-00-6$ & PCB-118 & 7 & 2 \\
\hline Qualitative & $\mathrm{C}_{12} \mathrm{H}_{4} \mathrm{Cl} 6$ & $35065-27-1$ & PCB-153 & 7 & 2 \\
\hline Qualitative & $\mathrm{C}_{12} \mathrm{H}_{5} \mathrm{Cl}_{5}$ & $32598-14-4$ & PCB-105 & 5 & 2 \\
\hline Qualitative & $\mathrm{C}_{12} \mathrm{H}_{4} \mathrm{Cl} 6$ & $35065-28-2$ & PCB-138 & 7 & 2 \\
\hline Qualitative & $\mathrm{C}_{12} \mathrm{H}_{4} \mathrm{Cl} 6$ & $52663-72-6$ & PCB-167 & 7 & 2 \\
\hline Qualitative & $\mathrm{C}_{12} \mathrm{H}_{4} \mathrm{Cl} 6$ & $38380-08-4$ & PCB-156 & 7 & 2 \\
\hline Qualitative & $\mathrm{C}_{12} \mathrm{H}_{4} \mathrm{Cl} 6$ & $69782-90-7$ & PCB-157 & 7 & 2 \\
\hline Qualitative & $\mathrm{C}_{12} \mathrm{H}_{3} \mathrm{Cl}_{7}$ & $35065-29-3$ & PCB-180 & 7 & 2 \\
\hline Qualitative & $\mathrm{C}_{12} \mathrm{H}_{6} \mathrm{Br}_{4} \mathrm{O}$ & $189084-61-5$ & $2,3^{\prime}, 4^{\prime}, 6$-Tetrabromodiphenyl ether (PBDE\# 47) & 7 & 2 \\
\hline Qualitative & $\mathrm{C}_{12} \mathrm{H}_{3} \mathrm{Cl}_{7}$ & $35065-30-6$ & PCB-170 & 7 & 2 \\
\hline Qualitative & $\mathrm{C}_{12} \mathrm{H}_{3} \mathrm{Cl}_{7}$ & $39635-31-9$ & PCB-189 & 7 & 2 \\
\hline Qualitative & $\mathrm{C}_{12} \mathrm{H}_{5} \mathrm{Br}_{5} \mathrm{O}$ & $93703-48-1$ & $2,2^{\prime}, 3,4,4^{\prime}$-Pentabromodiphenyl ether (PBDE\#100) & 7 & 2 \\
\hline Qualitative & $\mathrm{C}_{12} \mathrm{H}_{2} \mathrm{Cl} 8$ & $52663-78-2$ & PCB-195 & 7 & 3 \\
\hline Qualitative & $\mathrm{C}_{12} \mathrm{H}_{2} \mathrm{Cl} 8$ & $35694-08-7$ & PCB-194 & 7 & 2 \\
\hline Qualitative & $\mathrm{C}_{12} \mathrm{H}_{5} \mathrm{Br} 5 \mathrm{O}$ & $60348-60-9$ & $2,2^{\prime}, 4,4^{\prime}, 6$-Pentabromodiphenyl ether (PBDE\#99) & 7 & 2 \\
\hline Qualitative & $\mathrm{C}_{12} \mathrm{HClg}$ & 40186-72-9 & PCB-206 & 7 & 2 \\
\hline Qualitative & $\mathrm{C}_{12} \mathrm{Cl}_{10}$ & $2051-24-3$ & PCB-209 & 7 & 2 \\
\hline Qualitative & $\mathrm{C}_{12} \mathrm{H}_{4} \mathrm{Br} 6 \mathrm{O}$ & $68631-49-2$ & $2,2^{\prime}, 4,4^{\prime}, 5,5^{\prime}$-Hexabromodiphenyl ether (PBDE\#154) & 7 & 2 \\
\hline \multirow[t]{4}{*}{ Qualitative } & $\mathrm{C}_{15} \mathrm{H}_{12} \mathrm{Br}_{4} \mathrm{O}_{2}$ & $79-94-7$ & Tetrabromobisphenol A (TBBP-A) & 7 & 2 \\
\hline & $\mathrm{C}_{14} \mathrm{H}_{2} \mathrm{~N}_{2} \mathrm{O}_{4}$ & $187227-45-8$ & Oseltamivircarbonsäure & 1 & 3 \\
\hline & $\mathrm{C}_{15} \mathrm{H}_{12} \mathrm{~N}_{2} \mathrm{O}_{3}$ & $2784-27-2$ & 5-(4-hydroxyphenyl)-5-phenylhydantoin & 1 & 3 \\
\hline & $\mathrm{C}_{14} \mathrm{H}_{29} \mathrm{NO}_{7} \mathrm{Si}$ & $192389-48-3$ & diethyl N-[3-(trimethoxysilyl)propyl]aspartate & 1 & 3 \\
\hline
\end{tabular}


Continued

\begin{tabular}{|c|c|c|c|c|c|}
\hline Conc (ng/g) & $\begin{array}{l}\text { Molecular } \\
\text { Formula }\end{array}$ & CAS & Common name & $\begin{array}{l}\text { Occur/ } \\
\text { matrix }\end{array}$ & $\begin{array}{r}\text { ID } \\
\text { Level }\end{array}$ \\
\hline & $\mathrm{C}_{9} \mathrm{H}_{1} 6 \mathrm{O}_{3}$ & $65593-73-9$ & Methyl (cyclohexyloxy)acetate & 1 & 3 \\
\hline & $\mathrm{C}_{17} \mathrm{H}_{24} \mathrm{O}$ & $13171-00-1$ & Celestolide & 1 & 3 \\
\hline & $\mathrm{C}_{10} \mathrm{H}_{10 O}$ & $529-34-0$ & Tetralinone & 1 & 3 \\
\hline & $\mathrm{C}_{15} \mathrm{H}_{14} \mathrm{O} 6$ & $154-23-4$ & catechin & 1 & 3 \\
\hline & & $843-55-0$ & bisfenol Z & 3 & 1 \\
\hline & & $1478-61-1$ & hexafluoroBPA (bisfenol AF) & 3 & 1 \\
\hline & & $123-08-0$ & 4-hydroxybenzaldehyd & 6 & 2 \\
\hline & & $1074-86-8$ & 4-formyl indole & 6 & 3 \\
\hline & & $60-32-2$ & aminocaproic acid & 4 & 3 \\
\hline
\end{tabular}


Table 29: Fish Norway

\begin{tabular}{|c|c|c|c|c|c|}
\hline Conc (ng/g) & $\begin{array}{l}\text { Molecular } \\
\text { Formula }\end{array}$ & CAS & Common name & $\begin{array}{l}\text { Occur/ } \\
\text { matrix }\end{array}$ & $\begin{array}{r}\text { ID } \\
\text { Level }\end{array}$ \\
\hline 3442,34 & $\mathrm{C}_{14} \mathrm{H}_{2} 6 \mathrm{O}_{2}$ & $544-64-9$ & Myristoleic acid & 6 & 2 \\
\hline 2484,13 & $\mathrm{C}_{17} \mathrm{H}_{3} 6 \mathrm{O}_{2} \mathrm{Si}$ & $18603-17-3$ & Tetradecanoic acid, trimethylsilyl ester & 7 & 2 \\
\hline 1925,69 & $\mathrm{C}_{7} \mathrm{H}_{5} \mathrm{NS}$ & $95-16-9$ & Benzothiazole & 5 & 2 \\
\hline 1691,36 & $\mathrm{C}_{23} \mathrm{H}_{4} \mathrm{OO}_{3}$ & $71041-49-1$ & 11-(3,4-Dimethyl-5-pentyl-2-furyl)-dodecanoic acid, methyl ester & 6 & 2 \\
\hline 549,96 & $\mathrm{C}_{7} \mathrm{H}_{6} \mathrm{O}_{2}$ & $65-85-0$ & Benzoic acid & 7 & 2 \\
\hline 545,81 & $\mathrm{C}_{10 H} \mathrm{OH}_{16}$ & $3760-14-3$ & 1,5-Cyclooctadiene, 1,5-dimethyl- & 3 & 2 \\
\hline 469,71 & $\mathrm{C}_{1} 9 \mathrm{H}_{4} \mathrm{OO}_{2} \mathrm{Si}$ & $55520-89-3$ & Hexadecanoic acid, trimethylsilyl ester & 7 & 2 \\
\hline 458,54 & $\mathrm{C}_{7} \mathrm{H}_{5} \mathrm{NS}$ & $272-16-2$ & 1,2-Benzisothiazole & 3 & 2 \\
\hline 442,79 & $\mathrm{C}_{21} \mathrm{H}_{3} 6 \mathrm{O}_{3}$ & $86879-46-1$ & 11-(3,4-Dimethyl-5-propyl-2-furyl)-undecanoic acid, methyl ester & 6 & 2 \\
\hline 440,55 & $\mathrm{C}_{23} \mathrm{H}_{3} 6 \mathrm{O}_{4}$ & - & Phthalic acid, hept-3-yl octyl ester & 2 & 2 \\
\hline 424,42 & $\mathrm{C}_{1} 8 \mathrm{H}_{3} 8 \mathrm{O}_{2} \mathrm{Si}$ & $74367-22-9$ & n-Pentadecanoic acid, trimethylsilyl ester & 6 & 2 \\
\hline 415,40 & $\mathrm{C}_{7} \mathrm{H}_{5} \mathrm{NS}_{2}$ & $149-30-4$ & 2-Mercaptobenzothiazole & 1 & 2 \\
\hline 326,98 & $\mathrm{C}_{8} \mathrm{H}_{12} \mathrm{O}$ & $30086-02-3$ & 3,5-Octadien-2-one, $(E, E)$ - & 7 & 2 \\
\hline 166,29 & $\mathrm{C}_{12} \mathrm{H}_{4} \mathrm{Cl} 6$ & $74472-43-8$ & $2,3,3^{\prime}, 4,5^{\prime}, 6$-Hexachloro-1,1'-biphenyl & 4 & 2 \\
\hline 148,49 & $\mathrm{C}^{2} \mathrm{H}_{1} 6 \mathrm{O}$ & $5715-23-1$ & 3,4-Dimethylcyclohexanol & 6 & 2 \\
\hline 144,94 & $\mathrm{C}_{1} 6 \mathrm{H}_{33} \mathrm{NO}$ & $629-54-9$ & Hexadecanamide & 4 & 2 \\
\hline 131,82 & $\mathrm{C}_{11} \mathrm{H}_{18} \mathrm{O}_{4}$ & - & Fumaric acid, ethyl 3-methylbut-2-yl ester & 7 & 2 \\
\hline 121,41 & $\mathrm{C}_{1} 8 \mathrm{H}_{2} 6 \mathrm{O}$ & $1222-05-5$ & Galaxolide & 4 & 2 \\
\hline 117,15 & $\mathrm{C}_{13} \mathrm{H}_{2} \mathrm{OO}$ & $14901-07-6$ & 3-Buten-2-one, 4-(2,6,6-trimethyl-1-cyclohexen-1-yl)- & 4 & 2 \\
\hline 104,92 & $\mathrm{C}_{14} \mathrm{H}_{14} \mathrm{O}$ & $19578-70-2$ & Benzene, 1-methyl-2-(phenylmethoxy)- & 3 & 2 \\
\hline 101,64 & $\mathrm{C}_{1} \mathrm{OH}_{14} \mathrm{O}_{2}$ & $1948-33-0$ & t-Butylhydroquinone & 5 & 3 \\
\hline 76,43 & $\mathrm{C}_{12} \mathrm{H}_{4} \mathrm{Cl} 6$ & $52663-72-6$ & 1,1'-Biphenyl, 2,3',4,4',5,5'-hexachloro- & 2 & 2 \\
\hline 55,96 & $\mathrm{C}_{12} \mathrm{H}_{24} \mathrm{O}_{3}$ & $74367-34-3$ & Propanoic acid, 2-methyl-, 3-hydroxy-2,4,4-trimethylpentyl ester & 7 & 2 \\
\hline 51,44 & $\mathrm{C}_{15} \mathrm{H}_{13} \mathrm{~N}$ & $2286-54-6$ & Benzenepropanenitrile, á-phenyl- & 1 & 2 \\
\hline 51,30 & $\mathrm{C}_{12} \mathrm{H}_{5} \mathrm{Cl}_{5}$ & $52663-62-4$ & 1,1'-Biphenyl, 2,2',3,3',4-pentachloro- & 1 & 2 \\
\hline 45,74 & $\mathrm{C}_{12} \mathrm{H}_{5} \mathrm{Cl}_{5}$ & $32598-14-4$ & 1,1'-Biphenyl, 2,3,3',4,4'-pentachloro- & 2 & 2 \\
\hline 40,41 & $\mathrm{C}_{9} \mathrm{H}_{10 O}$ & $140-11-4$ & Acetic acid, phenylmethyl ester & 3 & 2 \\
\hline 37,94 & $\mathrm{C}_{9} \mathrm{H}_{10} \mathrm{O}_{3}$ & $10139-84-1$ & $2^{\prime}, 4^{\prime}-$ Dihydroxy-3'-methylacetophenone & 5 & 2 \\
\hline 37,34 & $\mathrm{C}_{12} \mathrm{H}_{6} \mathrm{Cl}_{4}$ & $35693-99-3$ & 1,1'-Biphenyl, 2,2',5,5'-tetramethyl- & 7 & 3 \\
\hline $34,5^{8}$ & $\mathrm{C} 8 \mathrm{H}_{6} \mathrm{O}_{2}$ & $1074-12-0$ & Phenylglyoxal & 5 & 2 \\
\hline 27,84 & $\mathrm{C} 9 \mathrm{H} 8 \mathrm{O}$ & $4432-63-7$ & 2-Phenylpropenal & 4 & 2 \\
\hline 21,60 & $\mathrm{C}_{12} \mathrm{H}_{5} \mathrm{Cl}_{5}$ & $55215-17-3$ & 1,1'-Biphenyl, 2,2',3,4,6-Pentachloro- & 3 & 2 \\
\hline 20,67 & $\mathrm{C}_{12} \mathrm{H}_{3} \mathrm{Cl}_{7}$ & $35065-30-6$ & 1,1'-Biphenyl, 2,2',3,3',4,4',5-heptachloro- & 2 & 2 \\
\hline 19,98 & $\mathrm{C}_{14} \mathrm{H}_{10}$ & $85-01-8$ & Phenanthrene & 2 & 2 \\
\hline 18,17 & $\mathrm{C}_{5} \mathrm{H}_{9} \mathrm{NO}_{2}$ & $4394-85-8$ & N-Formylmorpholine & 7 & 2 \\
\hline 16,73 & $\mathrm{C}_{12} \mathrm{H}_{12} \mathrm{O}_{5}$ & $5062-30-6$ & Phenacylidene diacetate & 4 & 2 \\
\hline 12,64 & $\mathrm{C}_{9} \mathrm{H}_{1} 8 \mathrm{Cl}_{3} \mathrm{O}_{4} \mathrm{P}$ & $13674-84-5$ & 2-Propanol, 1-chloro-, phosphate (3:1) & 5 & 2 \\
\hline 11,36 & $\mathrm{C}_{15} \mathrm{H}_{22} \mathrm{O}_{3}$ & - & Salicylic acid, butyl ether, butyl ester & 5 & 2 \\
\hline 10,79 & $\mathrm{C}_{8} \mathrm{H}_{7} \mathrm{NS}_{2}$ & $615-22-5$ & Benzothiazole, 2-(methylthio)- & 3 & 2 \\
\hline 7,94 & $\mathrm{C}^{2} \mathrm{H}_{10 O}$ & $123-07-9$ & Phenol, 4-ethyl- & 1 & 2 \\
\hline Qualitative & $\mathrm{C}_{6} \mathrm{H}_{4} \mathrm{Br}_{2} \mathrm{O}$ & $615-58-7$ & 2,4-dibromophenol & 7 & 2 \\
\hline Qualitative & $\mathrm{C}_{7} \mathrm{H}_{5} \mathrm{Br}_{3}$ & $607-99-8$ & 2,4,6-tribromoanisole & 7 & 2 \\
\hline Qualitative & $\mathrm{C}_{6} \mathrm{H}_{3} \mathrm{Br}_{3} \mathrm{O}$ & $118-79-6$ & $2,4,6$-Tribromophenol & 7 & 2 \\
\hline Qualitative & $\mathrm{C}_{12} \mathrm{H} 8 \mathrm{Br} 2 \mathrm{O}$ & $147217-71-8$ & 2,4'-Dibromodiphenyl ether (PBDE\#7) & 4 & 2 \\
\hline Qualitative & - & - & Natural product, $\mathrm{Br}_{3} \mathrm{Cl}_{3}$, probably monoterpene & 4 & 5 \\
\hline Qualitative & $\mathrm{C}_{12} \mathrm{H}_{5} \mathrm{Cl}_{5}$ & $31508-00-6$ & PCB-118 & 7 & 2 \\
\hline Qualitative & $\mathrm{C}_{12} \mathrm{H}_{4} \mathrm{Cl} 6$ & $35065-27-1$ & PCB-153 & 7 & 2 \\
\hline Qualitative & $\mathrm{C}_{12} \mathrm{H}_{5} \mathrm{Cl}_{5}$ & $32598-14-4$ & PCB-105 & 5 & 2 \\
\hline Qualitative & $\mathrm{C}_{12} \mathrm{H}_{4} \mathrm{Cl} 6$ & $35065-28-2$ & PCB-138 & 7 & 2 \\
\hline Qualitative & $\mathrm{C}_{12} \mathrm{H}_{4} \mathrm{Cl} 6$ & $52663-72-6$ & PCB-167 & 7 & 2 \\
\hline Qualitative & $\mathrm{C}_{12} \mathrm{H}_{4} \mathrm{Cl} 6$ & $38380-08-4$ & PCB-156 & 7 & 2 \\
\hline Qualitative & $\mathrm{C}_{12} \mathrm{H}_{4} \mathrm{Cl} 6$ & $69782-90-7$ & PCB-157 & 7 & 2 \\
\hline Qualitative & $\mathrm{C}_{12} \mathrm{H}_{3} \mathrm{Cl}_{7}$ & $35065-29-3$ & PCB-180 & 7 & 2 \\
\hline Qualitative & $\mathrm{C}_{12} \mathrm{H}_{6} \mathrm{Br}_{4} \mathrm{O}$ & $189084-61-5$ & $2,3^{\prime}, 4^{\prime}, 6$-Tetrabromodiphenyl ether (PBDE\#47) & 7 & 2 \\
\hline Qualitative & $\mathrm{C}_{12} \mathrm{H}_{3} \mathrm{Cl}_{7}$ & $35065-30-6$ & PCB-170 & 7 & 2 \\
\hline Qualitative & $\mathrm{C}_{12} \mathrm{H}_{3} \mathrm{Cl}_{7}$ & $39635-31-9$ & PCB-189 & 7 & 2 \\
\hline Qualitative & $\mathrm{C}_{12} \mathrm{H}_{5} \mathrm{Br}_{5} \mathrm{O}$ & $93703-48-1$ & $2,2^{\prime}, 3,4,4^{\prime}$-Pentabromodiphenyl ether (PBDE\#100) & 7 & 2 \\
\hline Qualitative & $\mathrm{C}_{12} \mathrm{H}_{2} \mathrm{Cl} 8$ & $52663-78-2$ & PCB-195 & 7 & 3 \\
\hline Qualitative & $\mathrm{C}_{12} \mathrm{H}_{2} \mathrm{Cl} 8$ & $35694-08-7$ & PCB-194 & 7 & 2 \\
\hline Qualitative & $\mathrm{C}_{12} \mathrm{H}_{5} \mathrm{Br}_{5} \mathrm{O}$ & $60348-60-9$ & $2,2^{\prime}, 4,4^{\prime}, 6$-Pentabromodiphenyl ether (PBDE\#99) & 7 & 2 \\
\hline Qualitative & $\mathrm{C}_{12} \mathrm{HCl}_{9}$ & $40186-72-9$ & PCB-206 & 7 & 2 \\
\hline Qualitative & $\mathrm{C}_{12} \mathrm{Cl}_{10}$ & $2051-24-3$ & PCB-209 & 7 & 2 \\
\hline Qualitative & $\mathrm{C}_{12} \mathrm{H}_{4} \mathrm{Br} 6 \mathrm{O}$ & $68631-49-2$ & $2,2^{\prime}, 4,4^{\prime}, 5,5^{\prime}$-Hexabromodiphenyl ether (PBDE\#154) & 7 & 2 \\
\hline
\end{tabular}


Continued

\begin{tabular}{|c|c|c|c|c|c|}
\hline Conc (ng/g) & $\begin{array}{l}\text { Molecular } \\
\text { Formula }\end{array}$ & CAS & Common name & $\begin{array}{l}\text { Occur/ } \\
\text { matrix }\end{array}$ & $\begin{array}{r}\text { ID } \\
\text { Level }\end{array}$ \\
\hline \multirow[t]{16}{*}{ Qualitative } & \multirow[t]{16}{*}{$\mathrm{C}_{15} \mathrm{H}_{12} \mathrm{Br}_{4} \mathrm{O}_{2}$} & $79-94-7$ & Tetrabromobisphenol A (TBBP-A) & 7 & 2 \\
\hline & & $58-63-9$ & Inosine & 2 & 3 \\
\hline & & $89-39-4$ & 1,4-dimethoxy-2-nitrobenzene & 1 & 3 \\
\hline & & $477-93-0$ & dimethoxanate & 1 & 3 \\
\hline & & $692-04-6$ & acetyllysine & 1 & 3 \\
\hline & & $26088-14-2$ & 9,9-bis(4-hydroxyphenyl)fluorene & 1 & 3 \\
\hline & & $6786-83-0$ & $\begin{array}{l}\alpha, \alpha \text {-bis }[4 \text {-(dimethylamino)phenyl]-4-(phenylamino)naphthalene- } \\
\text { 1-methanol }\end{array}$ & & \\
\hline & & $186817-80-1$ & Propanoic acid, 2-hydroxy-, 2-ethylhexyl ester, (2S)- & & \\
\hline & & $147-94-4$ & cytarabine & 1 & 3 \\
\hline & & $93-83-4$ & N,N-bis(2-hydroxyethyl)oleamide & 1 & 3 \\
\hline & & $81-24-3$ & taurocholic acid & 2 & 3 \\
\hline & & $1478-61-1$ & hexafluoroBPA (bisfenol AF) & 3 & 1 \\
\hline & & $13595-25-0$ & bisphenol M & 3 & 1 \\
\hline & & $550-24-3$ & embelin & 2 & 3 \\
\hline & & $123-08-0$ & 4-hydroxybenzaldehyde & 6 & 3 \\
\hline & & $60-32-2$ & aminocaproic acid & 4 & 3 \\
\hline
\end{tabular}


Table 30: Fish Greenland

\begin{tabular}{|c|c|c|c|c|c|}
\hline Conc (ng/g) & $\begin{array}{l}\text { Molecular } \\
\text { Formula }\end{array}$ & CAS & Common name & $\begin{array}{l}\text { Occur/ } \\
\text { matrix }\end{array}$ & $\begin{array}{r}\text { ID } \\
\text { Level }\end{array}$ \\
\hline 3269,82 & $\mathrm{C}_{14} \mathrm{H}_{2} 6 \mathrm{O}_{2}$ & $544-64-9$ & Myristoleic acid & 6 & 2 \\
\hline 1625,97 & $\mathrm{C}_{17} \mathrm{H}_{3} 6 \mathrm{O}_{2} \mathrm{Si}$ & $18603-17-3$ & Tetradecanoic acid, trimethylsilyl ester & 7 & 2 \\
\hline 1297,39 & $\mathrm{C}_{7} \mathrm{H}_{5} \mathrm{NS}$ & $272-16-2$ & 1,2-Benzisothiazole & 3 & 2 \\
\hline 523,11 & $\mathrm{C}_{1} 6 \mathrm{H}_{34} \mathrm{O}_{3}$ & $3055-93-4$ & Diethylene glycol monododecyl ether & 1 & 2 \\
\hline 440,01 & $\mathrm{C}_{8} \mathrm{H}_{1} 6 \mathrm{O}$ & $5715-23-1$ & 3,4-Dimethylcyclohexanol & 6 & 2 \\
\hline 422,28 & $\mathrm{C}_{2} 4 \mathrm{H}_{3} 8 \mathrm{O}_{4}$ & - & Phthalic acid, di(2-propylpentyl) ester & 3 & 2 \\
\hline 326,24 & $\mathrm{C}_{1} 9 \mathrm{H}_{4} \mathrm{OO}_{2} \mathrm{Si}$ & $55520-89-3$ & Hexadecanoic acid, trimethylsilyl ester & 7 & 2 \\
\hline 231,72 & $\mathrm{C}_{13} \mathrm{H}_{20} \mathrm{O}$ & $14901-07-6$ & 3-Buten-2-one, 4-(2,6,6-trimethyl-1-cyclohexen-1-yl)- & 4 & 2 \\
\hline 173,87 & $\mathrm{C}_{23} \mathrm{H}_{4} \mathrm{OO}_{3}$ & 71041-49-1 & 11-(3,4-Dimethyl-5-pentyl-2-furyl)-dodecanoic acid, methyl ester & 6 & 2 \\
\hline 164,00 & $\mathrm{C}_{8} \mathrm{H}_{12} \mathrm{O}$ & $30086-02-3$ & 3,5-Octadien-2-one, $(E, E)$ - & 7 & 2 \\
\hline 151,92 & $\mathrm{C}_{1} 6 \mathrm{H}_{33} \mathrm{NO}$ & $629-54-9$ & Hexadecanamide & 4 & 2 \\
\hline 144,94 & $\mathrm{C}_{1} 8 \mathrm{H}_{3} 8 \mathrm{O}_{2} \mathrm{Si}$ & $74367-22-9$ & n-Pentadecanoic acid, trimethylsilyl ester & 6 & 2 \\
\hline 142,85 & $\mathrm{C}_{13} \mathrm{H}_{2} \mathrm{OO}$ & $79-77-6$ & trans-á-lonone & 1 & 2 \\
\hline 136,39 & $\mathrm{C}_{1} \mathrm{OH}_{16}$ & $3760-14-3$ & 1,5-Cyclooctadiene, 1,5-dimethyl- & 3 & 3 \\
\hline 135,77 & $\mathrm{C}_{7} \mathrm{H}_{6} \mathrm{O}_{2}$ & $65-85-0$ & Benzoic acid & 7 & 2 \\
\hline 118,04 & $\mathrm{C}_{11} \mathrm{H}_{16} 6 \mathrm{O}_{2}$ & $17092-92-1$ & $2(4 \mathrm{H})$-Benzofuranone, 5,6,7,7a-tetrahydro-4,4,7a-trimethyl-, (R)- & 3 & 2 \\
\hline 100,13 & $\mathrm{C}_{11} \mathrm{H}_{18} 8 \mathrm{O}_{4}$ & - & Fumaric acid, ethyl 3-methylbut-2-yl ester & 7 & 2 \\
\hline 100,10 & $\mathrm{C}_{13} \mathrm{H}_{1} 6$ & $30364-38-6$ & Naphthalene, 1,2-dihydro-1,1,6-trimethyl- & 3 & 2 \\
\hline 62,84 & $\mathrm{C}_{13} \mathrm{H}_{18}$ & $30316-36-0$ & Naphthalene, 1,2,3,4-tetrahydro-1,6,8-trimethyl- & 1 & 2 \\
\hline 58,22 & $\mathrm{C}_{7} \mathrm{H}_{5} \mathrm{NOS}$ & $934-34-9$ & $2(3 \mathrm{H})$-Benzothiazolone & 3 & 3 \\
\hline 51,28 & $\mathrm{C}_{21} \mathrm{H}_{3} 6 \mathrm{O}_{3}$ & $86879-46-1$ & 11-(3,4-Dimethyl-5-propyl-2-furyl)-undecanoic acid, methyl ester & 6 & 2 \\
\hline 47,47 & $\mathrm{C}_{12} \mathrm{H}_{6 \mathrm{Cl}} 4$ & $35693-99-3$ & 1,1'-Biphenyl, 2,2',5,5'-tetramethyl- & 7 & 3 \\
\hline 32,00 & $\mathrm{C}_{12} \mathrm{H}_{24} \mathrm{O}_{3}$ & $74367-34-3$ & Propanoic acid, 2-methyl-, 3-hydroxy-2,4,4-trimethylpentyl ester & 7 & 2 \\
\hline 23,31 & $\mathrm{C} 8 \mathrm{H}_{6} \mathrm{O}_{2}$ & $1074-12-0$ & Phenylglyoxal & 5 & 2 \\
\hline 19,72 & $\mathrm{C}_{9} \mathrm{H}_{1} 8 \mathrm{Cl}_{3} \mathrm{O}_{4} \mathrm{P}$ & $13674-84-5$ & 2-Propanol, 1-chloro-, phosphate (3:1) & 5 & 2 \\
\hline 18,59 & $\mathrm{C}_{9} \mathrm{H}_{10 O} \mathrm{O}_{2}$ & $70-70-2$ & Paroxypropione & 5 & 2 \\
\hline 14,44 & $\mathrm{C}_{5} \mathrm{H}_{9} \mathrm{NO}_{2}$ & $4394-85-8$ & N-Formylmorpholine & 7 & 2 \\
\hline 10,50 & $\mathrm{C} 9 \mathrm{H} 8 \mathrm{O}$ & $4432-63-7$ & 2-Phenylpropenal & 4 & 2 \\
\hline 7,37 & $\mathrm{C}_{9} \mathrm{H}_{12} \mathrm{O}_{2}$ & $1125-21-9$ & 2,6,6-Trimethyl-2-cyclohexene-1,4-dione & 4 & 2 \\
\hline 3,97 & $\mathrm{C}_{17} \mathrm{H}_{34} \mathrm{O}$ & 55019-46-0 & Hexadecanal, 2-methyl- & 3 & 2 \\
\hline 2,87 & $\mathrm{C}_{15} \mathrm{H}_{24}$ & $242794-76-9$ & Bicyclo[5.2.0]nonane, 2-methylene-4,8,8-trimethyl-4-vinyl- & 3 & 2 \\
\hline Qualitative & $\mathrm{C}_{6} \mathrm{H}_{4} \mathrm{Br}_{2} \mathrm{O}$ & $615-58-7$ & 2,4-dibromophenol & 7 & 2 \\
\hline Qualitative & $\mathrm{C}_{7} \mathrm{H}_{5} \mathrm{Br}_{3}$ & $607-99-8$ & 2,4,6-tribromoanisole & 7 & 2 \\
\hline Qualitative & $\mathrm{C}_{6} \mathrm{H}_{3} \mathrm{Br}_{3} \mathrm{O}$ & $118-79-6$ & $2,4,6$-Tribromophenol & 7 & 2 \\
\hline Qualitative & $\mathrm{C}_{12} \mathrm{H} 8 \mathrm{Br} 2 \mathrm{O}$ & $147217-71-8$ & $2,4^{\prime}$-Dibromodiphenyl ether (PBDE\#7) & 4 & 2 \\
\hline Qualitative & $\mathrm{C}_{12} \mathrm{H}_{5} \mathrm{Cl}_{5}$ & $31508-00-6$ & PCB-118 & 7 & 2 \\
\hline Qualitative & $\mathrm{C}_{12} \mathrm{H}_{4} \mathrm{Cl} 6$ & $35065-27-1$ & PCB-153 & 7 & 2 \\
\hline Qualitative & $\mathrm{C}_{12} \mathrm{H}_{4} \mathrm{Cl} 6$ & $35065-28-2$ & PCB-138 & 7 & 2 \\
\hline Qualitative & $\mathrm{C}_{12} \mathrm{H}_{4} \mathrm{Cl} 6$ & $52663-72-6$ & PCB-167 & 7 & 2 \\
\hline Qualitative & $\mathrm{C}_{12} \mathrm{H}_{4} \mathrm{Cl} 6$ & $38380-08-4$ & PCB-156 & 7 & 2 \\
\hline Qualitative & $\mathrm{C}_{12} \mathrm{H}_{4} \mathrm{Cl} 6$ & $69782-90-7$ & PCB-157 & 7 & 2 \\
\hline Qualitative & $\mathrm{C}_{12} \mathrm{H}_{3} \mathrm{Cl}_{7}$ & $35065-29-3$ & PCB-180 & 7 & 2 \\
\hline Qualitative & $\mathrm{C}_{12} \mathrm{H}_{6 \mathrm{Br}} \mathrm{O}_{\mathrm{O}}$ & $189084-61-5$ & $2,3^{\prime}, 4^{\prime}, 6$-Tetrabromodiphenyl ether (PBDE\# 47) & 7 & 2 \\
\hline Qualitative & $\mathrm{C}_{12} \mathrm{H}_{3} \mathrm{Cl}_{7}$ & $35065-30-6$ & PCB-170 & 7 & 2 \\
\hline Qualitative & $\mathrm{C}_{12} \mathrm{H}_{3} \mathrm{Cl}_{7}$ & $39635-31-9$ & PCB-189 & 7 & 2 \\
\hline Qualitative & $\mathrm{C}_{12} \mathrm{H}_{5} \mathrm{Br} 5 \mathrm{O}$ & $93703-48-1$ & $2,2^{\prime}, 3,4,4^{\prime}$-Pentabromodiphenyl ether (PBDE\#100) & 7 & 2 \\
\hline Qualitative & $\mathrm{C}_{12} \mathrm{H}_{2} \mathrm{Cl} 8$ & $52663-78-2$ & PCB-195 & 7 & 3 \\
\hline Qualitative & $\mathrm{C}_{12} \mathrm{H}_{2} \mathrm{Cl} 8$ & $35694-08-7$ & PCB-194 & 7 & 2 \\
\hline Qualitative & $\mathrm{C}_{12} \mathrm{H}_{5} \mathrm{Br}_{5} \mathrm{O}$ & $60348-60-9$ & $2,2^{\prime}, 4,4^{\prime}, 6$-Pentabromodiphenyl ether (PBDE\#99) & 7 & 2 \\
\hline Qualitative & $\mathrm{C}_{12} \mathrm{HClg}_{9}$ & 40186-72-9 & PCB-206 & 7 & 2 \\
\hline Qualitative & $\mathrm{C}_{12} \mathrm{Cl}_{10}$ & $2051-24-3$ & PCB-209 & 7 & 2 \\
\hline Qualitative & $\mathrm{C}_{12} \mathrm{H}_{4} \mathrm{Br} 6 \mathrm{O}$ & $68631-49-2$ & $2,2^{\prime}, 4,4^{\prime}, 5,5^{\prime}$-Hexabromodiphenyl ether (PBDE\#154) & 7 & 2 \\
\hline \multirow[t]{10}{*}{ Qualitative } & $\mathrm{C}_{15} \mathrm{H}_{12} \mathrm{Br}_{4} \mathrm{O}_{2}$ & $79-94-7$ & Tetrabromobisphenol A (TBBP-A) & 7 & 2 \\
\hline & $\mathrm{C}_{11} \mathrm{H}_{14} \mathrm{O}_{3}$ & $4247-02-3$ & isobutyl 4-hydroxybenzoate & 1 & 3 \\
\hline & $\mathrm{C}_{1} 8 \mathrm{H}_{2} 6 \mathrm{O}_{3}$ & $5466-77-3$ & Ethylhexyl methoxycinnamate & 1 & 3 \\
\hline & $\mathrm{C}_{11} \mathrm{H}_{18} 8 \mathrm{O}_{4}$ & $90474-13-8$ & diethyl 1,2-cyclopentanedicarboxylate & 1 & 3 \\
\hline & $\mathrm{C}_{10 \mathrm{H}_{14} \mathrm{O}_{3}}$ & $59-47-2$ & mephenesin & 1 & 3 \\
\hline & $\mathrm{C}_{2} \mathrm{OH}_{2} 8 \mathrm{O}_{3}$ & $302-97-6$ & 3-oxoandrost-4-ene-17 $\beta$-carboxylic acid & 1 & 3 \\
\hline & $\mathrm{C}_{8} \mathrm{H}_{1} \mathrm{O}_{3}$ & $106-31-0$ & butyric anhydride & 1 & 3 \\
\hline & $\mathrm{C}_{14} \mathrm{H}_{1} 6 \mathrm{CIN}_{3} \mathrm{O}$ & $67129-08-2$ & $\begin{array}{l}\text { 2-chloro- } \mathrm{N} \text {-(2,6-dimethylphenyl)- } \mathrm{N} \text {-(1H-pyrazol-1-ylmethyl) } \\
\text { acetamide }\end{array}$ & & \\
\hline & $\mathrm{C}_{13} \mathrm{H}_{17} \mathrm{NO}_{4}$ & $1086384-49-7$ & $\mathrm{~N}$-(2,6-dimethyl-phenyl)- $\mathrm{N}$-(2-methoxy-ethyl)-oxalamic acid & & \\
\hline & $\mathrm{C}_{9} \mathrm{H}_{1} 6 \mathrm{O}_{2}$ & $5726-19-2$ & 2-methylcyclohexyl acetate & 1 & 3 \\
\hline
\end{tabular}


Continued

\begin{tabular}{|c|c|c|c|c|c|}
\hline Conc (ng/g) & $\begin{array}{l}\text { Molecular } \\
\text { Formula }\end{array}$ & CAS & Common name & $\begin{array}{l}\text { Occur/ } \\
\text { matrix }\end{array}$ & $\begin{array}{r}\text { ID } \\
\text { Level }\end{array}$ \\
\hline & $\mathrm{C}_{12} \mathrm{H}_{15} \mathrm{~N}_{5} \mathrm{O}_{5}$ & $75128-73-3$ & $\begin{array}{l}\text { 2-[(2-acetamido-6,9-dihydro-6-oxo-1H-purin-9-yl]methoxy]ethyl } \\
\text { acetate }\end{array}$ & & \\
\hline & $\mathrm{C}_{9} \mathrm{H}_{7} \mathrm{NO}$ & $148-24-3$ & quinolin-8-ol & 1 & \\
\hline & & $80-09-1$ & bisphenol S & 2 & 1 \\
\hline & & $1478-61-1$ & hexafluoroBPA (bisfenol AF) & 3 & 1 \\
\hline & & $843-55-0$ & bisfenol Z & 3 & 1 \\
\hline & & $13595-25-0$ & bisphenol M & 3 & 1 \\
\hline & & $1074-86-8$ & 4-formyl indole & 6 & 3 \\
\hline & & $60-32-2$ & aminocaproic acid & 4 & 3 \\
\hline & & $584-44-1$ & Thymotic acid & 2 & 3 \\
\hline
\end{tabular}


Table 31: Fish Faroe Island

\begin{tabular}{|c|c|c|c|c|c|}
\hline Conc (ng/g) & $\begin{array}{l}\text { Molecular } \\
\text { Formula }\end{array}$ & CAS & Common name & $\begin{array}{l}\text { Occur/ } \\
\text { matrix }\end{array}$ & $\begin{array}{r}\text { ID } \\
\text { Level }\end{array}$ \\
\hline 1847,23 & $\mathrm{C}_{17} \mathrm{H}_{3} 6 \mathrm{O}_{2} \mathrm{Si}$ & $18603-17-3$ & Tetradecanoic acid, trimethylsilyl ester & 7 & 2 \\
\hline 327,18 & $\mathrm{C}_{10} \mathrm{H}_{16}$ & $3760-14-3$ & 1,5-Cyclooctadiene, 1,5-dimethyl- & 3 & 3 \\
\hline 322,62 & $\mathrm{C}_{7} \mathrm{H}_{5} \mathrm{NS}$ & $95-16-9$ & Benzothiazole & 5 & 2 \\
\hline 320,67 & $\mathrm{C}_{2} 4 \mathrm{H}_{3} 8 \mathrm{O}_{4}$ & - & Phthalic acid, di(6-methylhept-2-yl) ester & 2 & 2 \\
\hline 268,32 & $\mathrm{C}_{11} \mathrm{H}_{18} \mathrm{O}_{4}$ & - & Fumaric acid, ethyl 3-methylbut-2-yl ester & 7 & 2 \\
\hline 163,14 & $\mathrm{C}_{8} \mathrm{H}_{12} \mathrm{O}$ & $30086-02-3$ & 3,5-Octadien-2-one, $(E, E)$ - & 7 & 2 \\
\hline 161,67 & $\mathrm{C}_{17} \mathrm{H}_{25} \mathrm{NO}_{4}$ & - & Succinic acid, 2-(dimethylamino)ethyl 4-isopropylphenyl ester & 5 & 2 \\
\hline 151,05 & $\mathrm{C}_{9} \mathrm{H}_{10 O}$ & $70-70-2$ & Paroxypropione & 5 & 2 \\
\hline 142,27 & $\mathrm{C}_{19} \mathrm{H}_{4} \mathrm{OO} 2 \mathrm{Si}$ & $55520-89-3$ & Hexadecanoic acid, trimethylsilyl ester & 7 & 2 \\
\hline 115,22 & $\mathrm{C}_{1} 8 \mathrm{H}_{30}$ & $2719-62-2$ & Benzene, (1-pentylheptyl)- & 2 & 2 \\
\hline 111,15 & $\mathrm{C}_{1} 8 \mathrm{H}_{3} 8 \mathrm{O}_{2} \mathrm{Si}$ & $74367-22-9$ & n-Pentadecanoic acid, trimethylsilyl ester & 6 & 2 \\
\hline 109,28 & $\mathrm{C}_{1} 8 \mathrm{H}_{2} 6 \mathrm{O}$ & $1222-05-5$ & Galaxolide & 4 & 2 \\
\hline 94,00 & $\mathrm{C}_{11} \mathrm{H}_{20}$ & $2958-76-1$ & Naphthalene, decahydro-2-methyl- & 2 & 2 \\
\hline 90,03 & $\mathrm{C}_{7} \mathrm{H}_{6} \mathrm{O}_{2}$ & $65-85-0$ & Benzoic acid & 7 & 2 \\
\hline 82,76 & $\mathrm{C}_{12} \mathrm{H}_{6} \mathrm{Cl}_{4}$ & $35693-99-3$ & 1,1'-Biphenyl, 2,2',5,5'-tetramethyl- & 7 & 3 \\
\hline 67,36 & $\mathrm{C}_{10 \mathrm{H}_{14} \mathrm{O}_{2}}$ & $1948-33-0$ & t-Butylhydroquinone & 5 & 3 \\
\hline 62,81 & $\mathrm{C}_{15} \mathrm{H}_{28}$ & $54832-83-6$ & ${ }_{1} \mathrm{H}$-Indene, octahydro-2,2,4,4,7,7-hexamethyl-, trans- & 2 & 3 \\
\hline 52,76 & $\mathrm{C}_{12} \mathrm{H}_{24} \mathrm{O}_{3}$ & $74367-34-3$ & Propanoic acid, 2-methyl-, 3-hydroxy-2,4,4-trimethylpentyl ester & 7 & 2 \\
\hline 47,90 & $\mathrm{C}_{17} \mathrm{H}_{28}$ & $4537-15-9$ & Benzene, (1-butylheptyl)- & 2 & 2 \\
\hline 43,62 & $\mathrm{C}_{8} \mathrm{H}_{1} \mathrm{~N}_{4} \mathrm{O}_{2}$ & $58-08-2$ & Caffeine & 1 & 3 \\
\hline 40,07 & $\mathrm{C}_{7} \mathrm{H}_{5} \mathrm{NS}$ & $272-16-2$ & 1,2-Benzisothiazole & 3 & 2 \\
\hline 37,14 & $\mathrm{C}_{14} \mathrm{H}_{10}$ & $85-01-8$ & Phenanthrene & 2 & 2 \\
\hline 33,89 & $\mathrm{C}_{11} \mathrm{H}_{20}$ & - & trans-Decalin, 2-methyl- & 2 & 2 \\
\hline 33,14 & $\mathrm{C}_{12} \mathrm{H}_{22}$ & $1008-80-6$ & Naphthalene, decahydro-2,3-dimethyl- & 2 & 3 \\
\hline 33,07 & $\mathrm{C}_{8} \mathrm{H}_{1} 6 \mathrm{O}$ & $5715-23-1$ & 3,4-Dimethylcyclohexanol & 6 & 2 \\
\hline 26,09 & $\mathrm{C}_{5} \mathrm{H}_{9} \mathrm{NO}_{2}$ & $4394-85-8$ & N-Formylmorpholine & 7 & 2 \\
\hline 25,97 & $\mathrm{C}_{15} \mathrm{H}_{24}$ & $242794-76-9$ & Bicyclo[5.2.0]nonane, 2-methylene-4,8,8-trimethyl-4-vinyl- & 3 & 2 \\
\hline 23,82 & $\mathrm{C}_{12} \mathrm{H}_{22}$ & $66660-41-1$ & 2-Ethyldecahydronaphthalene, $(\mathrm{Z}, \mathrm{E})$ - & 2 & 2 \\
\hline 12,25 & $\mathrm{C}_{15} \mathrm{H}_{22} \mathrm{O}_{3}$ & - & Salicylic acid, butyl ether, butyl ester & 5 & 2 \\
\hline 8,91 & $\mathrm{C}_{17} \mathrm{H}_{34} \mathrm{O}$ & $55019-46-0$ & Hexadecanal, 2-methyl- & 3 & 2 \\
\hline Qualitative & $\mathrm{C}_{6} \mathrm{H}_{4} \mathrm{Br}_{2} \mathrm{O}$ & $615-58-7$ & 2,4-dibromophenol & 7 & 2 \\
\hline Qualitative & $\mathrm{C}_{7} \mathrm{H}_{5} \mathrm{Br}_{3}$ & $607-99-8$ & 2,4,6-tribromoanisole & 7 & 2 \\
\hline Qualitative & $\mathrm{C}_{6} \mathrm{H}_{3} \mathrm{Br}_{3} \mathrm{O}$ & $118-79-6$ & $2,4,6$-Tribromophenol & 7 & 2 \\
\hline Qualitative & $\mathrm{C}_{12} \mathrm{H} 8 \mathrm{Br} 2 \mathrm{O}$ & $147217-71-8$ & 2,4'-Dibromodiphenyl ether (PBDE\#7) & 4 & 2 \\
\hline Qualitative & - & - & Natural product, $\mathrm{Br}_{3} \mathrm{Cl}_{3}$, probably monoterpene & 4 & 5 \\
\hline Qualitative & $\mathrm{C}_{12} \mathrm{H}_{4} \mathrm{Cl} 6$ & $35065-27-1$ & PCB-153 & 7 & 2 \\
\hline Qualitative & $\mathrm{C}_{12} \mathrm{H}_{4} \mathrm{Cl} 6$ & $35065-28-2$ & PCB-138 & 7 & 2 \\
\hline Qualitative & $\mathrm{C}_{12} \mathrm{H}_{4} \mathrm{Cl} 6$ & $52663-72-6$ & PCB-167 & 7 & 2 \\
\hline Qualitative & $\mathrm{C}_{12} \mathrm{H}_{4} \mathrm{Cl} 6$ & $38380-08-4$ & PCB-156 & 7 & 2 \\
\hline Qualitative & $\mathrm{C}_{12} \mathrm{H}_{4} \mathrm{Cl} 6$ & $69782-90-7$ & PCB-157 & 7 & 2 \\
\hline Qualitative & $\mathrm{C}_{12} \mathrm{H}_{3} \mathrm{Cl}_{7}$ & $35065-29-3$ & PCB-18o & 7 & 2 \\
\hline Qualitative & $\mathrm{C}_{12} \mathrm{H}_{6 \mathrm{Br}} \mathrm{O}$ & $189084-61-5$ & $2,3^{\prime}, 4^{\prime}, 6$-Tetrabromodiphenyl ether (PBDE\# 47) & 7 & 2 \\
\hline Qualitative & $\mathrm{C}_{12} \mathrm{H}_{3} \mathrm{Cl}_{7}$ & $35065-30-6$ & PCB-170 & 7 & 2 \\
\hline Qualitative & $\mathrm{C}_{12} \mathrm{H}_{3} \mathrm{Cl}_{7}$ & $39635-31-9$ & PCB-189 & 7 & 2 \\
\hline Qualitative & $\mathrm{C}_{12} \mathrm{H}_{5} \mathrm{Br}_{5} \mathrm{O}$ & $93703-48-1$ & $2,2^{\prime}, 3,4,4^{\prime}$-Pentabromodiphenyl ether (PBDE\#100) & 7 & 2 \\
\hline Qualitative & $\mathrm{C}_{12} \mathrm{H}_{2} \mathrm{Cl} 8$ & $52663-78-2$ & PCB-195 & 7 & 3 \\
\hline Qualitative & $\mathrm{C}_{12} \mathrm{H}_{2} \mathrm{Cl} 8$ & $35694-08-7$ & PCB-194 & 7 & 2 \\
\hline Qualitative & $\mathrm{C}_{12} \mathrm{H}_{5} \mathrm{Br}_{5} \mathrm{O}$ & $60348-60-9$ & $2,2^{\prime}, 4,4^{\prime}, 6$-Pentabromodiphenyl ether (PBDE\#99) & 7 & 2 \\
\hline Qualitative & $\mathrm{C}_{12} \mathrm{HCl}_{9}$ & 40186-72-9 & PCB-206 & 7 & 2 \\
\hline Qualitative & $\mathrm{C}_{12} \mathrm{Cl}_{10}$ & $2051-24-3$ & PCB-209 & 7 & 2 \\
\hline Qualitative & $\mathrm{C}_{12} \mathrm{H}_{4} \mathrm{Br} 6 \mathrm{O}$ & $68631-49-2$ & $2,2^{\prime}, 4,4^{\prime}, 5,5^{\prime}$-Hexabromodiphenyl ether (PBDE\#154) & 7 & 2 \\
\hline \multirow[t]{10}{*}{ Qualitative } & $\mathrm{C}_{15} \mathrm{H}_{12} \mathrm{Br}_{4} \mathrm{O}_{2}$ & $79-94-7$ & Tetrabromobisphenol A (TBBP-A) & 7 & 2 \\
\hline & & $976-70-5$ & 3-oxopregn-4-ene-21,17a-carbolactone & 1 & 3 \\
\hline & & $23680-84-4$ & 2-chloro-6,7-dimethoxyquinazolin-4-amine & 1 & 3 \\
\hline & & $14035-33-7$ & 3,5-Di-tert-butyl-4-hydroxyacetophenone & 1 & 3 \\
\hline & & $363-24-6$ & dinoprostone & 1 & 3 \\
\hline & & $108-59-8$ & dimethyl malonate & 1 & 3 \\
\hline & & $34434-80-5$ & Abiet-8(14)-en-18-oic acid & 2 & 3 \\
\hline & & $61443-78-5$ & Amoxapine-8-hydroxy & 1 & 3 \\
\hline & & $26856-96-2$ & nonyl hydrogen sulfate & 1 & 3 \\
\hline & & $80-09-1$ & bisphenol S & 2 & 1 \\
\hline
\end{tabular}


Continued

\begin{tabular}{l|l} 
Conc (ng/g) & $\begin{array}{l}\text { Molecular } \\
\text { Formula }\end{array}$
\end{tabular}

13595-25-0 
Nordic Council of Ministers

Nordens Hus

Ved Stranden 18

DK-1061 Copenhagen K

www.norden.org

\section{SUSPECT SCREENING IN NORDIC COUNTRIES}

This report describes the results of a suspect screening study in samples from city areas in the Nordic countries. In contrast to target analysis, suspect screening starts with a general sample preparation and identification including as much compounds as possible. The resulting list of recorded compounds will be identified by comparison with a list of suspected compounds. Suspect screening has shown to be very useful for identification of emerging environmental pollutants. It was possible to identify: Perfluorinated compounds (PFC), chlorinated and brominated compounds, flame retardants, bisphenols, polycyclic aromatic compounds $(P A C)$, industrial additives, and pharmaceuticals and personal care products (PPCP). This study was jointly performed by NILU, NIVA, and Umeå University on behalf of the Nordic Council of Ministers. The study was supervised by the Nordic screening group. 